Universidade de São Paulo

Instituto de Física

\title{
Orientação óptica de spin em semicondutores magnéticos - calcógenos de európio
}

\section{Giovanni Decot Galgano}

Orientador: Prof. Dr. André Bohomoletz Henriques

Tese de doutorado apresentada ao

Instituto de Física para a obtenção do título de Doutor em Ciências

São Paulo 
FICHA CATALOGRÁFICA

Preparada pelo Serviço de Biblioteca e Informação do Instituto de Física da Universidade de São Paulo

Galgano, Giovanni Decot

Orientação óptica de spin em semicondutores magnéticos - calcógenos de európio. - São Paulo, 2012.

Tese (Doutorado) - Universidade de São Paulo. Instituto de Física - Depto. de Física dos Materiais e Mecânica.

Orientador: Prof. Dr. André Bohomoletz Henriques

Área de Concentração: Física

Unitermos: 1. Física do estado sólido; 2. Magnetismo; 3. Magneto-óptica; 4. Espectroscopia óptica;

5. Fotoluminescência. 


\section{Giovanni Decot Galgano}

\section{Orientação óptica de spin em semicondutores magnéticos - calcógenos de európio}

Tese apresentada ao Instituto de Física da Universidade de São Paulo para obtenção do título de Doutor em Ciências

Área de concentração: materiais magnéticos e propriedades magnéticas

Orientador

Prof. Dr. André Bohomoletz Henriques Banca Examinadora Prof. Dr. Flavio Orlando Plentz Filho Prof. Dr. Gilmar Eugênio Marques Prof. Dr. Günther Bauer Prof. Dr. Leandro Hostalácio Freire de Andrade

São Paulo 

Aos meus pais Cristina e Nicola, às minhas irmãs Paula e Aurélia e à minha namorada Jaqueline. 



\section{Agradecimentos}

Ao Prof. Dr. André Bohomoletz Henriques, pela orientação no doutorado e pelas inúmeras lições que contribuíram para meu desenvolvimento profissional e pessoal.

Aos Profs. Dr. Eduardo Abramof e Gunther Bauer pelo fornecimento das amostras estudadas.

Ao Instituto de Física da USP, pela oportunidade da realização do doutorado.

À CNPq pela bolsa de estudos.

Aos Profs. Drs. Valdir Bindilatti, Antônio Domingues dos Santos e Fernando Navarra pelo apoio no decorrer do trabalho.

Aos funcionários do Departamento de Física dos Materiais e Mecânica por manter a infraestrutura fundamental para o desenvolvimento do trabalho.

Aos meu pais, Nicola Stefano Galgano e Cristina Decot, por terem me criado e me educado com muita dedicação e amor e por fornecerem condições imprescindíveis para a realização deste trabalho.

Às minhas irmãs Paula Decot Galgano e Aurélia Decot Galgano pelo apoio e carinho incondicionais.

A toda a minha família, que sempre me incentivou.

À minha namorada Jaqueline Medeiros Padovan, por seu amor e dedicação.

Ao colega e bom amigo Victor A. N. Righetti.

Aos colegas e amigos do IF pela agradável companhia.

A todos os amigos, que sempre proporcionaram muitas alegrias.

Aos amigos Luis Marcelo Rosso e Danielle E. Bumajny por me receberem em suas casas.

E a todos que de alguma maneira contribuíram para a realização deste trabalho. 


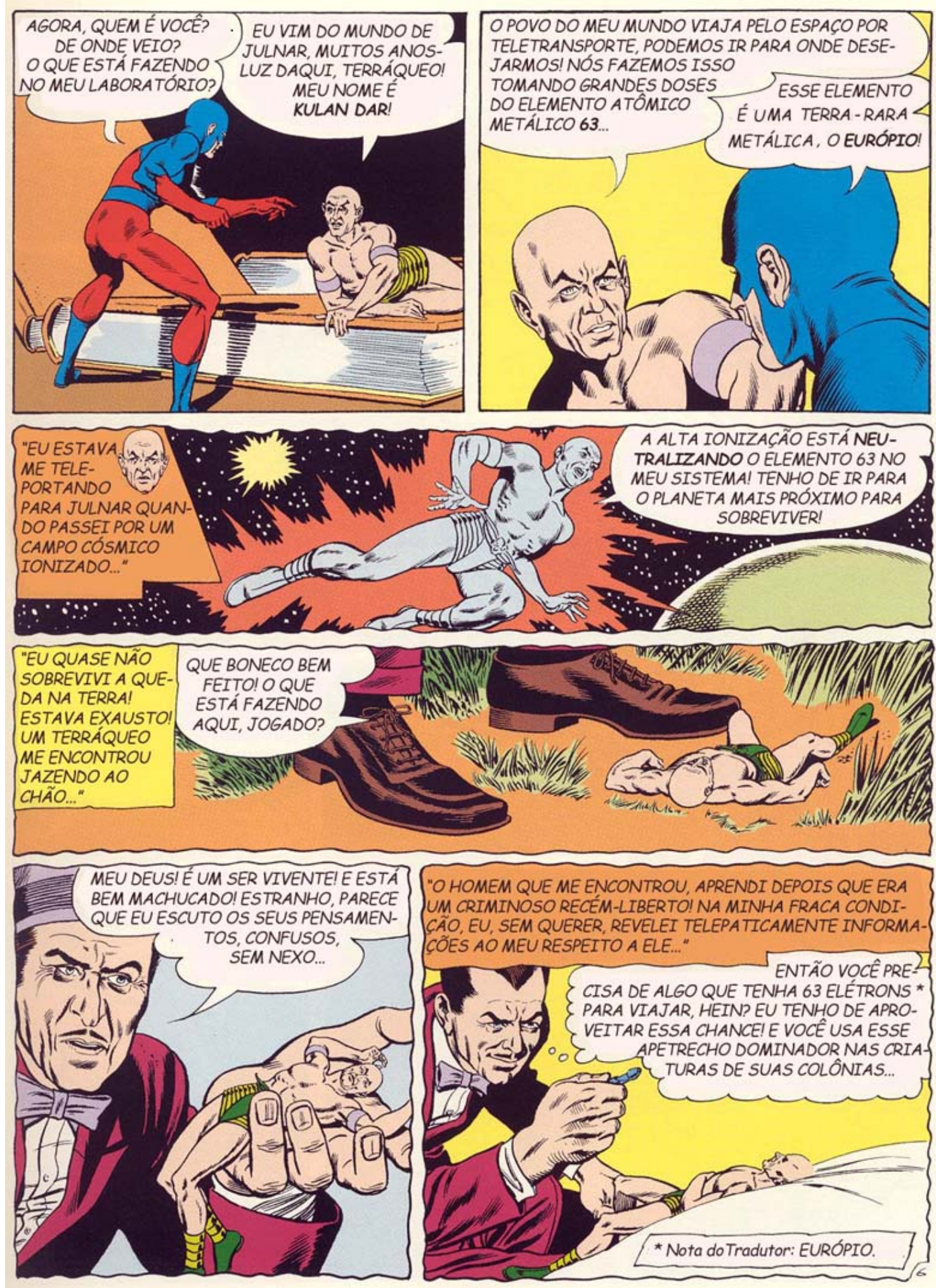

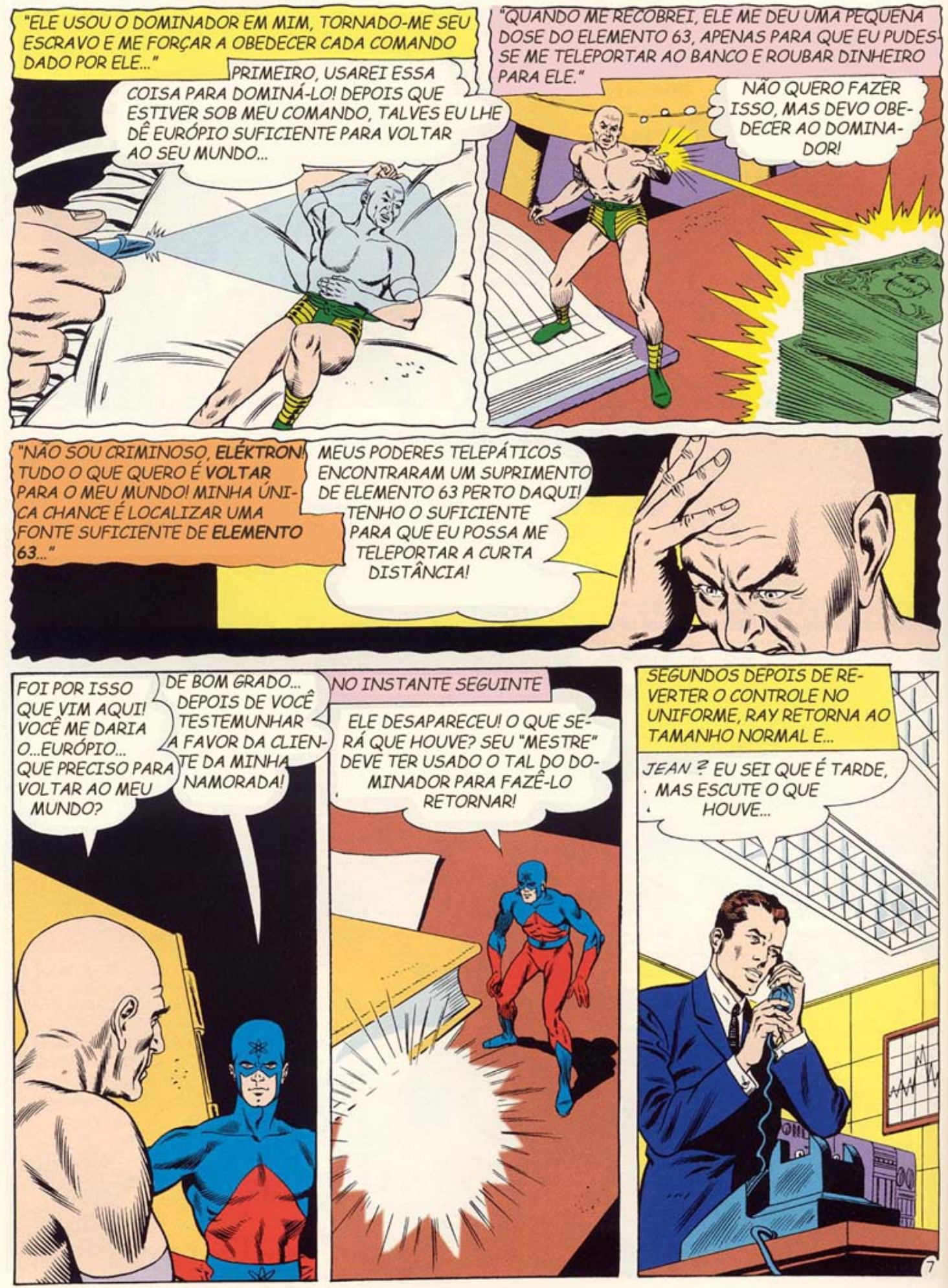

Gardner Fox e Gil Kane, revista Showcase n 34 (1961) 


\section{Resumo}

A investigação das propriedades ópticas e sua relação com as propriedades magnéticas dos semicondutores é de grande interesse para a comunidade científica, em virtude da enorme demanda por novas tecnologias e funcionalidades que podem surgir dessas pesquisas. Os calcógenos de európio são semicondutores intrinsecamente magnéticos, transparentes na região visível do espectro eletromagnético e integráveis em matrizes de silício e nitreto de gálio, sendo assim fortes candidatos a aplicações tecnológicas envolvendo magnetismo e óptica. Neste trabalho são investigados os espectros de absorção e fotoluminescência dos calcógenos de európio, com base no modelo $4 f \rightarrow 5 d\left(t_{2 g}\right)$ da transição óptica de dipolo elétrico, o qual mostrou-se totalmente adequado para a descrição da absorção óptica nos calcógenos de európio em função do campo magnético aplicado, explicando a presença de linhas de absorção estreitas e dicróicas nos espectros em campo alto e a forma larga dos espectros de absorção em campo nulo. Nos espectros de fotoluminescência do EuTe, entretanto, foram detectados estados eletrônicos não contemplados pelo modelo $4 f \rightarrow 5 d\left(t_{2 g}\right)$, em especial uma banda de emissão denominada $\mathrm{MX}_{0}$, acoplada a modos vibracionais da rede. Uma linha zero-fônon correspondente a uma transição que não produz fônons pôde ser bem definida e a partir do deslocamento dessa linha em função do campo magnético foi possível detectar inequivocamente a formação de polarons magnéticos no EuTe pela primeira vez; o raio polarônico foi estimado como $R=3.6 a$, onde $a$ é o parâmetro de rede e a energia de ligação desse polaron foi estimada em $E_{p}=45 \mathrm{meV}$, um resultado que foi confirmado através de medidas do deslocamento da linha zero-fônon em função da temperatura. Adicionalmente procurou-se identificar o estado eletrônico associado à emissão $\mathrm{MX}_{0}$ : a partir de medidas da intensidade da fotoluminescência em função da potência de excitação foi possível sugerir que a emissão $M X_{0}$ provem de estados eletrônicos ligados a defeitos da rede e foi possível estimar a concentração desses defeitos como menor que 0.1 ppm.

Palavras-chave: semicondutores magnéticos, calcógenos de európio, absorção óptica, fotoluminescência, polarons magnéticos. 



\begin{abstract}
Investigation of optical properties and their relation to magnetic properties of semiconductors is of great interest to scientific community, due to the large demand for new technologies and features that can arise from these studies. Europium chalcogenides are intrinsically magnetic semiconductors, transparent in the visible region of electromagnetic spectrum and integrable into silicon and gallium nitride matrices, beeing strong candidates for technological applications involving magnetism and optics. The present study investigates absorption and photoluminescence spectra of europium chalcogenides, based on the $4 f \rightarrow 5 d\left(t_{2 g}\right)$ model of the electric dipole optical transition, which proved to be entirely appropriate to describe polarized optical absorption in europium chalcogenides as a function of magnetic field, explaining the presence of narrow dichroic lines at high fields and the broad shape of the zero-field absorption spectrum. However, in photoluminescence spectra of EuTe, electronic states not covered by the $4 f \rightarrow 5 d\left(t_{2 g}\right)$ model were detected, in particular an emission band labeled $\mathrm{MX}_{0}$, which is coupled to vibrational modes of the lattice. A transition without production of phonons, corresponding to a zero-phonon line, could be well resolved and from the displacement of the zero-phonon line as a function of magnetic field the formation of magnetic polarons in EuTe could be detected unambiguously for the first time. The polaronic radius is estimated as $R=3.6 a$, where $a$ is the lattice parameter, and the polaron binding energy is estimated as $E_{p}=45 \mathrm{meV}$, a result that was confirmed by measurements of zero-phonon line displacement as a function of temperature. Additionally, we sought to identify the electronic state associated with $\mathrm{MX}_{0}$ emission: from measurements of the photoluminescence intensity as a function of excitation power, it was possible to suggest that $\mathrm{MX}_{0}$ emission comes from an electronic state coupled to lattice defects of low concentration, which we estimate to be of less than $0.1 \mathrm{ppm}$.

Keywords: magnetic semiconductors, europium chalcogenides, optical absorption, photoluminescence, magnetic polarons.

Note: an English version of this thesis is also available. Please get in contact to me or go to http://romeo.if.usp.br/ ggalgano/Thesis.pdf.
\end{abstract}





\section{Conteúdo}

1 Introdução $\quad 17$

2 Fundamentos teóricos $\quad 21$

2.1 A interação de exchange . . . . . . . . . . . . . . . . . 21

2.1 .1 Oátomo de He . . . . . . . . . . . . . . 23

2.1.2 Hamiltoniano de Heisenberg . . . . . . . . . . . . . . 27

2.2 O modelo do campo efetivo para o magnetismo . . . . . . . . . . 30

2.2.1 Partículas carregadas num campo magnético uniforme . . . . . . 31

2.2.2 Átomos num campo magnético efetivo . . . . . . . . . . . . . 44

2.2.3 Outros tipos de magnetismo . . . . . . . . . . . . . 52

2.3 Magnetismo nos Calcógenos de Európio . . . . . . . . . . . . . 53

2.3.1 Calcógenos de európio . . . . . . . . . . . . . . 53

2.3.2 Modelo do campo médio para o magnetismo nos EuX . . . . . 56

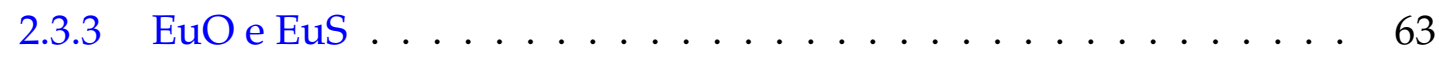

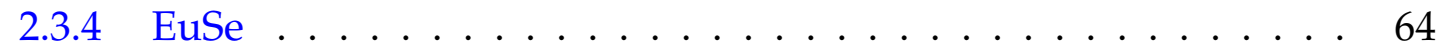

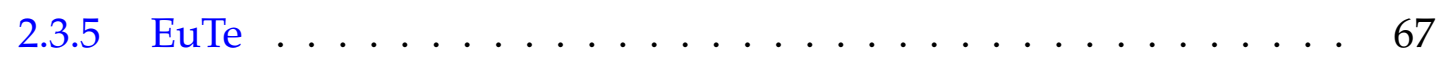

$\begin{array}{lll}3 & \text { Materiais e métodos } & 73\end{array}$

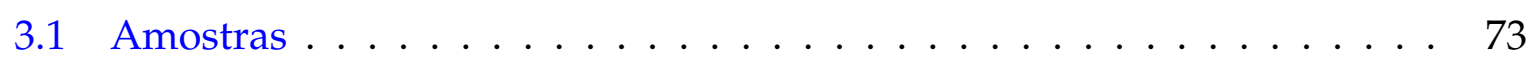

3.2 Arranjo experimental . . . . . . . . . . . . . . . . 74

3.2.1 Montagem para absorção . . . . . . . . . . . . . . . . 74

3.2.2 Montagem para fotoluminescência . . . . . . . . . . . . . . 77 
4.1 Modelo para a absorção óptica nos EuX . . . . . . . . . . . . . . . 83

4.1.1 Propagação de ondas eletromagnéticas em um dielétrico . . . . . 84

4.1 .2 Polarização da luz . . . . . . . . . . . . . . . . 86

4.1 .3 Absorção óptica por um material . . . . . . . . . . . . . . . . 88

4.1.4 Probabilidade de transição de um elétron . . . . . . . . . . . . . 92

4.1.5 Transição óptica num sistema de muitos elétrons . . . . . . . . . . 97

4.1 .6 Níveis de energia dos EuX . . . . . . . . . . . . . . . 98

4.1.7 Cálculo do coeficiente de absorção nos EuX . . . . . . . . . . . . . 109

4.1.8 Espectros de absorção dos EuX . . . . . . . . . . . . . . 126

4.2 Espectros de absorção no EuTe e EuSe . . . . . . . . . . . . . . . . 135

4.2.1 Identificação das fases magnéticas no EuSe pelo deslocamento da linhas de absorção . . . . . . . . . . . . . . . . . . . . 141

4.3 Espectros de Fotoluminescência do EuTe . . . . . . . . . . . . . 145

4.3.1 Polaron magnético no EuTe . . . . . . . . . . . . . . 156

4.3.2 Deslocamento das linhas de absorção e PL em termos da magnetização . . . . . . . . . . . . . . . . . . 159

4.3.3 Evidências da formação de polarons magnéticos em EuTe a partir de um estudo da ZPL em função da temperatura . . . . . . . . . 161

4.3.4 A natureza do estado $X \ldots \ldots$. . . . . . . . . . . . . 172

5 Conclusões $\quad 181$

$\begin{array}{ll}\text { Bibliografia } & 191\end{array}$

A Lista de publicações relacionadas à tese 193 


\section{Capítulo 1}

\section{Introdução}

A Física de semicondutores é uma área que estuda as propriedades desses materiais, no intuito de aproveitar seus mecanismos e funcionalidades em aplicações tecnológicas. Essa área da Física consolidou-se fortemente a partir de 1950 [1], quando a descoberta do transístor bipolar revolucionou a eletrônica, permitindo posteriormente a criação dos circuitos integrados e dos computadores, chegando ao estágio atual onde é muito difícil imaginar o dia-a-dia do homem sem a presença dos recursos computacionais baseados em semicondutores nos mais variados setores.

A chegada da era digital trouxe novos paradigmas tecnológicos, como a otimização e a miniaturização dos dispositivos eletrônicos; a crescente utilização e produção desses dispositivos vem trazendo uma enorme demanda por novos recursos e funcionalidades. Os softwares estão cada vez mais potentes e complexos, exigindo unidades de processamento mais eficientes e com capacidade cada vez maior. Entretanto, a tecnologia atual aproxima-se de um limite intrínseco para a miniaturização dos dispositivos, imposto pelo tamanho finito dos átomos que constituem os processadores, de forma que em certo momento será impossível, ou economicamente inviável, reduzir o tamanho de um processador mantendo a sua capacidade. Na busca de dispositivos mais eficientes que superem o limite da miniaturização, novas tecnologias computacionais são procuradas para além da eletrônica convencional. Uma das áreas que surgiu nesse processo é a spintrônica [2,3], onde procura-se utilizar os graus de liberdade de spin 
do elétron para manipular e controlar informações em um circuito. Os semicondutores magnéticos ganharam especial notoriedade desde então [4], atraindo muitas pesquisas científicas para essa área.

Os calcógenos de európio, EuX, onde Xé um dos calcogênios $\mathrm{O}, \mathrm{S}$, Se ou Te, são uma família de semicondutores intrinsecamente magnéticos, fabricados pela primeira vez em 1939 [5] e que vem sendo bastante estudada desde os anos 1960 [6, 7], quando foram previstas potenciais aplicações envolvendo magnetismo para compostos de terras-raras [8]. Nos anos seguintes, foram estudadas as propriedades ópticas dos EuX [9] e foi demonstrado que o controle da polarização do spins nos EuX poderia ser empregado em dispositivos magneto-ópticos [10]. Mais recentemente foram desenvolvidos filtros de spin de alta eficiência baseados em calcógenos de európio [11] e também foi demonstrado que os EuX podem ser integrados em matrizes de nitreto de gálio e silício [12], o semicondutor mais amplamente difundido, aumentando a perspectiva da aplicação dos EuX em dispositivos spintrônicos. Os EuX também já foram implementados nas novas estruturas materiais que surgiram com o aprimoramento das técnicas de crescimento; alguns exemplos são a fabricação de nanopartículas ferromagnéticas de EuS [13, 14, 15] e superantiferromagnéticas de EuTe [16] e também o crescimento de estruturas de baixa dimensionalidade com EuX, como ilhas quânticas de EuSe [17] e EuTe [18], atualmente temas de grande interesse para a comunidade científica.

Para compreender as diversas propriedades de um semicondutor, é necessário conhecer a sua estrutura eletrônica. Conhecendo os estados eletrônicos associados à banda de valência e à banda de condução de um semicondutor, é possível fazer previsões sobre a condutividade, sobre as propriedades ópticas e as propriedades magnéticas desse material. A determinação dos primeiros estados excitados dos EuX é um tema que vem sendo desenvolvido desde o fim dos anos 1960, quando um esquema dos níveis de energia dos EuX foi montado a partir do ciclo de Born-Haber foi proposto por Wachter [19] e Kasuya [20]. Nesse esquema, foi determinado que os primeiros estados de energia superior à dos níveis $4 f$ são estados onde o elétron fotoexcitado ocupa orbitais $6 s$ ou $5 d$. Alguns pesquisadores como Busch, Wachter e Güntherodt procuraram 
determinar a estrutura eletrônica dos EuX a partir de investigações ópticas, reforçando a conclusão de que a banda de valência é composta pelo orbital $4 f^{7}$ e a banda de condução é composta por estados $5 d$ ou $6 s$ dos átomos de Eu, sub a ação de um campo cristalino de simetria octaédrica. Entretanto, como as regras de seleção implicam que não podem haver transições $4 f \rightarrow 6$ s por dipolo elétrico, o modelo que buscou-se utilizar é o da transição de dipólo elétrico $4 f \rightarrow 5 d\left(t_{2 g}\right)$, que a grosso modo foi capaz de explicar algumas observações magneto-ópticas, como a rotação de Faraday e o espectro de absorção dos EuX. Entretanto, essa transição prevê linhas estreitas para o limiar da absorção, o que não fora observado nos resultados experimentais da época [9]. A discrepância entre o modelo $4 f \rightarrow 5 d\left(t_{2 g}\right)$ de um único íon e as observações experimentais da época, foi atribuída por Güntherodt a "efeitos de muitos corpos" (referência [21]), implicando que as correlações entre os elétrons não poderiam ser desprezadas, como é feito no modelo $4 f \rightarrow 5 d\left(t_{2 g}\right)$.

Mais recentemente, o surgimento de novas técnicas de crescimento, como a epitaxia de feixe molecular (MBE), com as quais é possível produzir amostras de alta pureza e excelente qualidade estrutural, associado à demanda por semicondutores magnéticos para aplicações tecnológicas envolvendo magnetismo, reativou o interesse da comunidade científica pelos EuX. Com amostras epitaxiais de EuTe e EuSe, foram realizados experimentos de absorção óptica em função do campo magnético com controle de polarização [22, 23, 24, 25], os quais revelaram um par de linhas dicróicas estreitas na fase ferromagnética, que desapareciam em campo nulo. Uma re-examinação do modelo $4 f \rightarrow 5 d\left(t_{2 g}\right)$ de um elétron - com a inclusão da formação de domínios magnéticos nos EuX, ignorada até então - permitiu uma modelagem completa dos espectros de absorção dos EuX em função do campo magnético, a qual demonstrou total concordância com os resultados experimentais [26, 27], contribuindo para a consolidação definitiva do modelo de transição $4 f \rightarrow 5 d\left(t_{2 g}\right)$ na descrição do limiar da absorção nos EuX. A partir dessa nova formulação foi possível explicar o dicroísmo circular magnético [26], o dicroísmo linear magnético [28] e ainda prever propriedade ópticas não-lineares nos EuX, cuja teoria apresentada na referência [29] mostrou boa adequação na descrição 
dos resultados experimentais de geração de segundos e terceiros harmônicos em EuTe e EuSe [30, 31, 32, 33].

Com base no modelo da transição $4 f \rightarrow 5 d\left(t_{2 g}\right)$, investigamos neste trabalho a correlação entre a incidência de radiação e o magnetismo nos EuX. Inicialmente serão discutidos os conceitos fundamentais que regem o magnetismo nos materiais em geral. Em seguida o magnetismo dos EuX será modelado na aproximação do campo médio, a qual mostra-se bastante adequada para descrever a maioria das propriedades magnéticas dos EuX, que são considerados "realizações do modelo de Heisenberg do magnetismo" [34]. Por fim são apresentados resultados experimentais dos espectros de absorção do EuTe e EuSe e dos espectros de fotoluminescência do EuTe, onde são evidenciadas as correlações entre a luz e o magnetismo nesses materiais, com dois resultados diferentes em especial: o primeiro é a possibilidade de detectar os estados magnéticos dos EuX através de seus espectros ópticos e o segundo é a possibilidade de criar, através da incidência de radiação no EuTe, uma região de spins polarizados, ou um polaron magnético, o que é uma proposta antiga [35, 36], que contudo foi confirmada inequivocamente pela primeira vez através dos resultados obtidos nos espectros de fotoluminescência do EuTe em função do campo magnético e da temperatura. 


\section{Capítulo 2}

\section{Fundamentos teóricos}

\subsection{A interação de exchange}

A interação de exchange tem sua origem mais fundamental na interação eletromagnética entre dois corpos. Entre outras coisas, ela é o mecanismo responsável pela ligação covalente e pelo magnetismo de longo alcance em sólidos, o qual é um dos temas desenvolvidos ao longo deste trabalho. Essa interação surge naturalmente do mecanismo quântico de átomos e moléculas, onde o grau de liberdade de spin desempenha papel fundamental na configuração dos estados possíveis para um sistema de partículas interagentes.

O spin de uma partícula ou corpo apresenta-se sob a forma de múltiplos discretos de uma quantidade conhecida como a constante de Planck reduzida, $\hbar=1.055 \times 10^{-34} \mathrm{Js}$, uma constante da natureza. Partículas cujo spin apresente múltiplos inteiros de $\hbar$ são classificadas como bósons e partículas com múltiplos semi-inteiros, isto é, 1/2, 3/2, 5/2, e assim por diante, são classificadas como férmions. O elétron, por exemplo, é classificado como férmion, pois seu spin vale $\hbar / 2$; outras partículas de spin semi-inteiro são os prótons e os nêutrons. O fóton, a partícula de luz, possui spin igual a $\hbar$ e portanto é um bóson, assim como o átomo de hidrogênio, que tem um spin total igual à soma do spin do próton $(\hbar / 2)$ e do elétron $(\hbar / 2)$, totalizando $S=\hbar$.

Na mecânica quântica toda partícula também possui um caráter ondulatório e pode 
ser descrita através de uma função de onda. Um sistema de $N$ partículas, onde cada partícula é descrita por uma função de onda, em geral pode ser descrito por uma função de onda total, composta por uma combinação das $N$ funções de onda individuais. Quando trata-se de partículas idênticas, como no caso de elétrons num átomo, deve-se levar em conta que essas partículas são indistinguíveis. Por serem indistinguíveis, o intercâmbio entre duas partículas não deve alterar o sistema, então diz-se que esse sistema apresenta simetria de intercâmbio, ou simetria de exchange, na forma derivada da língua inglesa. Essa simetria manifesta-se diretamente na função de onda total do sistema, que deve permanecer inalterada quando efetua-se o intercâmbio entre um par de partículas idênticas. Em alguns sistemas, o intercâmbio entre um par de partículas idênticas fornece uma função de onda idêntica à original, porém com sinal trocado. Esses sistemas também apresentam um tipo simetria de intercâmbio, uma vez que os eventos reais são descritos pelo módulo ao quadrado da função de onda. A função de onda nesses casos é dita antissimétrica.

Um dos postulados da mecânica quântica estabelece que em um sistema de férmions idênticos, a função de onda total deve ser obrigatoriamente antissimétrica por exchange e em um sistema de bósons idênticos, a função de onda total deve ser simétrica por exchange. Uma importante consequência da antissimetria da função de onda total para um sistema de férmions idênticos é o princípio da exclusão de Pauli, o qual estabelece que dois elétrons não podem ocupar o mesmo estado ao mesmo tempo. Esse princípio, enunciado por Wolfgang Pauli em 1925 está por trás de toda a estrutura eletrônica dos átomos e moléculas, e também está intrinsecamente relacionado com a interação eletrostática entre elétrons num átomo, como será mostrado a seguir.

Um sistema simples onde pode ser exemplificada a interação entre dois elétrons em um átomo é o átomo de hélio neutro. Utilizando o formalismo hamiltoniano da mecânica quântica numa abordagem variacional, será demonstrado que a interação coulombiana entre os dois elétrons e o princípio da exclusão de Pauli originam uma diferença de energia entre os possíveis estados de spin, a qual é intitulada energia de exchange, a qual pode ser formulada em termos de uma interação de exchange. 


\subsubsection{O átomo de He}

O átomo de hélio neutro é constituído por dois elétrons que movem-se no campo central do núcleo, que é composto por dois prótons e dois nêutrons. Com boa aproximação, o centro de massa desse átomo é o próprio núcleo, que será tratado como uma partícula puntiforme de carga positiva com módulo $2 e$, onde $e=-1.602 \times 10^{-19}$ C é a carga elementar. No referencial do centro de massa, cada elétron $i$, onde $i=1$ ou 2 , é situado por um vetor posição $\boldsymbol{r}_{i}$ e está sujeito ao potencial nuclear dado por

$$
V_{i}=-\frac{2 k e^{2}}{\left|\boldsymbol{r}_{i}\right|}
$$

onde $k=\frac{1}{4 \pi \epsilon_{0}}=8.988 \times 10^{9} \mathrm{~F} / \mathrm{m}$ é a constante de Coulomb do vácuo. Além do potencial atrativo do núcleo, os dois elétrons sofrem uma repulsão mútua, dada pelo potencial

$$
V_{2,1}=\frac{k e^{2}}{\left|\boldsymbol{r}_{2}-\boldsymbol{r}_{1}\right|}
$$

A energia total desse sistema é definida como a soma das energias cinética e potencial de cada elemento. A energia cinética do elétron $i$ é dada por $\boldsymbol{p}_{i}^{2} / 2 m_{e}$, onde $m_{e}$ é a massa do elétron e $\boldsymbol{p}$ o seu momento generalizado. A energia potencial é descrita em termos do potencial atrativo do núcleo (2.1) e do potencial repulsivo (2.2) devido ao segundo elétron. No referencial do núcleo, as energias cinética e potencial do mesmo não fazem parte da energia total. Nesse referencial o hamiltoniano quântico do sistema é construído a partir da energia total fazendo-se $\boldsymbol{p}_{i} \rightarrow-i \hbar \nabla$ para obter-se

$$
\mathcal{H}\left(\boldsymbol{r}_{1}, \boldsymbol{r}_{2}\right)=\frac{\hbar^{2}}{2 m_{e}}\left(\nabla_{1}^{2}+\nabla_{2}^{2}\right)-2 k e^{2}\left(\frac{1}{\left|\boldsymbol{r}_{1}\right|}+\frac{1}{\left|\boldsymbol{r}_{2}\right|}-\frac{1}{2\left|\boldsymbol{r}_{2}-\boldsymbol{r}_{1}\right|}\right)
$$

Os estados estacionários do sistema são determinados pela solução da equação de Schrödinger com esse hamiltoniano

$$
\mathcal{H} \Psi_{n}=E_{n} \Psi_{n}
$$

onde $\Psi_{n}$ é o $n$-ésimo autoestado do hamiltoniano, com correspondente autovalor $E_{n}$.

Encontrar as funções de onda que satisfazem (2.4) com o hamiltoniano (2.3) é um problema muito complicado, em virtude da presença do potencial de interação entre 
os elétrons $V_{2,1}$, que impede a separação de variáveis. Ainda assim, é possível procurar soluções em termos de uma função de onda com um parâmetro variacional [37], onde as variáveis possam ser separadas

$$
\Psi\left(\boldsymbol{r}_{1} s_{1}, \boldsymbol{r}_{2} \boldsymbol{s}_{2}\right)=\psi_{1}\left(\boldsymbol{r}_{1} s_{1}\right) \psi_{2}\left(\boldsymbol{r}_{2} s_{2}\right)
$$

Pelo fato dos dois elétrons serem indistinguíveis, a função de onda total deve obedecer o princípio de antissimetrização. Isso é possível em duas situações: a parte espacial da função de onda é simétrica e a parte de spin é antissimétrica, correspondendo a um singleto

$$
\Psi\left(\boldsymbol{r}_{1} \boldsymbol{s}_{1}, \boldsymbol{r}_{2} \boldsymbol{s}_{2}\right)=\frac{\psi_{1}\left(\boldsymbol{r}_{1}\right) \psi_{2}\left(\boldsymbol{r}_{2}\right)+\psi_{1}\left(\boldsymbol{r}_{2}\right) \psi_{2}\left(\boldsymbol{r}_{1}\right)}{\sqrt{2}} \times \frac{\alpha\left(\boldsymbol{s}_{1}\right) \beta\left(s_{2}\right)-\alpha\left(s_{2}\right) \beta(s 1)}{\sqrt{2}}
$$

ou a parte espacial é antissimétrica e a parte de spin é o tripleto simétrico

$$
\Psi\left(\boldsymbol{r}_{1} \boldsymbol{s}_{1}, \boldsymbol{r}_{2} \boldsymbol{s}_{2}\right)=\frac{\psi_{1}\left(\boldsymbol{r}_{1}\right) \psi_{2}\left(\boldsymbol{r}_{2}\right)-\psi_{1}\left(\boldsymbol{r}_{2}\right) \psi_{2}\left(\boldsymbol{r}_{1}\right)}{\sqrt{2}} \times\left\{\begin{array}{l}
\alpha\left(\boldsymbol{s}_{1}\right) \alpha\left(\boldsymbol{s}_{2}\right) \\
\frac{\alpha\left(\boldsymbol{s}_{1}\right) \beta\left(\boldsymbol{s}_{2}\right)-\alpha\left(\boldsymbol{s}_{2}\right) \beta(s 1)}{\sqrt{2}} \\
\beta\left(\boldsymbol{s}_{1}\right) \beta\left(\boldsymbol{s}_{2}\right)
\end{array}\right.
$$

Como o hamiltoniano (2.3) não depende do spin, a energia dos estados estacionários pode ser obtida por

$$
E=\frac{1}{2} \int_{\tau}\left[\psi_{1}^{*}\left(\boldsymbol{r}_{1}\right) \psi_{2}^{*}\left(\boldsymbol{r}_{2}\right) \pm \psi_{1}^{*}\left(\boldsymbol{r}_{2}\right) \psi_{2}^{*}\left(\boldsymbol{r}_{1}\right)\right] \mathcal{H}\left(\boldsymbol{r}_{1}, \boldsymbol{r}_{2}\right)\left[\psi_{1}\left(\boldsymbol{r}_{1}\right) \psi_{2}\left(\boldsymbol{r}_{2}\right) \pm \psi_{1}\left(\boldsymbol{r}_{2}\right) \psi_{2}\left(\boldsymbol{r}_{1}\right)\right] d^{3} \tau
$$

onde $\tau$ representa todo o espaço.

O hamiltoniano (2.3) pode ser separado em dois termos de um elétron num potencial central e um terceiro termo de interação entre os elétrons

$$
\begin{gathered}
\mathcal{H}_{1}\left(\boldsymbol{r}_{1}\right)=\frac{\boldsymbol{p}_{1}^{2}}{2 m_{e}}-\frac{2 k e^{2}}{\left|\boldsymbol{r}_{1}\right|} \\
\mathcal{H}_{2}\left(\boldsymbol{r}_{2}\right)=\frac{\boldsymbol{p}_{2}^{2}}{2 m_{e}}-\frac{2 k e^{2}}{\left|\boldsymbol{r}_{2}\right|} \\
\mathcal{H}_{1,2}\left(\boldsymbol{r}_{1}, \boldsymbol{r}_{2}\right)=\frac{k e^{2}}{\left|\boldsymbol{r}_{2}-\boldsymbol{r}_{1}\right|}
\end{gathered}
$$


de forma que a solução da equação de Schrödinger (2.8) pode ser escrita como

$$
E=E_{1}+E_{2}+E_{1,2}
$$

onde

$$
\begin{aligned}
& E_{1}=\int \psi_{1}^{*}\left(\boldsymbol{r}_{1}\right) \mathcal{H}_{1} \psi_{1}\left(\boldsymbol{r}_{1}\right) d^{3} \tau_{1} \\
& E_{2}=\int \psi_{2}^{*}\left(\boldsymbol{r}_{2}\right) \mathcal{H}_{2} \psi_{2}\left(\boldsymbol{r}_{2}\right) d^{3} \tau_{2}
\end{aligned}
$$

são as energias de cada elétron sob a ação do potencial central e

$$
\begin{aligned}
E_{1,2}=\frac{1}{2} \int_{\tau}\left[\psi_{1}^{*}\left(\boldsymbol{r}_{1}\right) \psi_{2}^{*}\left(\boldsymbol{r}_{2}\right) \pm \psi_{1}^{*}\left(\boldsymbol{r}_{2}\right) \psi_{2}^{*}\left(\boldsymbol{r}_{1}\right)\right] \mathcal{H}_{1,2}\left(\boldsymbol{r}_{1}, \boldsymbol{r}_{2}\right) \times \\
\times\left[\psi_{1}\left(\boldsymbol{r}_{1}\right) \psi_{2}\left(\boldsymbol{r}_{2}\right) \pm \psi_{1}\left(\boldsymbol{r}_{2}\right) \psi_{2}\left(\boldsymbol{r}_{1}\right)\right] d^{3} \tau
\end{aligned}
$$

é a energia de interação entre os elétrons, a qual pode ser calculada por métodos aproximativos.

As energias $E_{1}$ e $E_{2}$ podem ser obtidas analiticamente pela solução de um átomo hidrogenoide de número atômico $Z$, que é o parâmetro variacional. A energia de interação é dada pela integral (2.15), a qual tem resultados diferentes para os casos em que a função de onda espacial é simétrica ou antissimétrica

$$
\begin{array}{r}
E_{1,2}^{S}=\frac{1}{2} \int_{\tau}\left[\psi_{1}^{*}\left(\boldsymbol{r}_{1}\right) \psi_{2}^{*}\left(\boldsymbol{r}_{2}\right)+\psi_{1}^{*}\left(\boldsymbol{r}_{2}\right) \psi_{2}^{*}\left(\boldsymbol{r}_{1}\right)\right] \mathcal{H}_{1,2}\left(\boldsymbol{r}_{1}, \boldsymbol{r}_{2}\right) \times \\
\times\left[\psi_{1}\left(\boldsymbol{r}_{1}\right) \psi_{2}\left(\boldsymbol{r}_{2}\right)+\psi_{1}\left(\boldsymbol{r}_{2}\right) \psi_{2}\left(\boldsymbol{r}_{1}\right)\right] d^{3} \tau \\
E_{1,2}^{A S}=\frac{1}{2} \int_{\tau}\left[\psi_{1}^{*}\left(\boldsymbol{r}_{1}\right) \psi_{2}^{*}\left(\boldsymbol{r}_{2}\right)-\psi_{1}^{*}\left(\boldsymbol{r}_{2}\right) \psi_{2}^{*}\left(\boldsymbol{r}_{1}\right)\right] \mathcal{H}_{1,2}\left(\boldsymbol{r}_{1}, \boldsymbol{r}_{2}\right) \times \\
\times\left[\psi_{1}\left(\boldsymbol{r}_{1}\right) \psi_{2}\left(\boldsymbol{r}_{2}\right)-\psi_{1}\left(\boldsymbol{r}_{2}\right) \psi_{2}\left(\boldsymbol{r}_{1}\right)\right] d^{3} \tau
\end{array}
$$

No estado fundamental, os dois elétrons ocupam o orbital 1s, de forma que a parte espacial das funções de onda é simétrica e a parte de spin é necessariamente o singleto antissimétrico. No primeiro estado excitado, um dos elétrons ocupa o orbital 1s e o outro elétron ocupa um orbital 2s. Nesse caso, a parte espacial da função de onda total pode assumir a forma simétrica ou antissimétrica. As energias $E_{1 s}$ e $E_{2 s}$ não dependem da simetria, entretanto as integrais (2.16) e (2.17) têm resultados diferentes nos dois 
casos

$$
\begin{aligned}
& E_{1,2}^{S}=I+J \\
& E_{1,2}^{A S}=I-J
\end{aligned}
$$

onde

$$
I=\int_{\tau_{1}} \int_{\tau_{2}}\left|\psi_{1 s}\left(\boldsymbol{r}_{1}\right)\right|^{2}\left|\psi_{2 s}\left(\boldsymbol{r}_{2}\right)\right|^{2} \frac{k e^{2}}{\left|\boldsymbol{r}_{2}-\boldsymbol{r}_{1}\right|} d^{3} \tau_{1} d^{3} \tau_{2}
$$

é chamada integral de Coulomb e

$$
J=\int_{\tau_{1}} \int_{\tau_{2}} \psi_{1 s}^{*}\left(\boldsymbol{r}_{1}\right) \psi_{2 s}^{*}\left(\boldsymbol{r}_{2}\right) \frac{k e^{2}}{\left|\boldsymbol{r}_{2}-\boldsymbol{r}_{1}\right|} \psi_{1 s}\left(\boldsymbol{r}_{2}\right) \psi_{2 s}\left(\boldsymbol{r}_{1}\right) d^{3} \tau_{1} d^{3} \tau_{2}
$$

é a integral de exchange.

A integral de exchange para o átomo de hélio na configuração $1 s 2 s$ vale aproximadamente $0.40 \mathrm{eV}$ (de acordo com [38] pg. 56). Como $J>0$, a energia do primeiro estado excitado, dada pelas equações (2.18) e (2.19), é menor quando a função de onda espacial é antissimétrica. Se a parte espacial da função de onda é antissimétrica, a parte de spin deve ser simétrica, portanto o tripleto de spin é mais estável, indicando que a orientação relativa entre os spins é paralela. Em outros sistemas, como por exemplo a molécula $\mathrm{H}_{2}^{+}$, a integral $J$ é negativa e a situação se inverte, sendo o singleto de spin a configuração de menor energia.

O cálculo dos estados excitados do átomo de hélio demonstrou que a partir da interação eletrostática entre os elétrons, surgem configurações preferenciais para a orientação dos spins. É interessante notar que as funções de onda de spin não entram diretamente no cálculo da energia de exchange, que é determinada pela separação em energia dos estados singleto e tripleto. Entretanto essa separação acarreta em um mínimo de energia do primeiro estado excitado do átomo de hélio neutro, quando os dois spins estão orientados paralelamente. Isso pode ser compreendido fisicamente pelo fato de que no estado singleto, a função de onda espacial é simétrica, e os elétrons tendem a ficar mais próximos um do outro, de forma que o efeito da repulsão eletrostática entre os dois elétrons é mais intenso no singleto. 
Uma formulação análoga pode ser feita para a interação entre elétrons de átomos diferentes, tanto em moléculas quanto em sólidos. A descrição de um orbital molecular como uma combinação linear de orbitais atômicos, é uma aproximação conhecida como tight-binding, onde a energia de exchange é proporcional ao recobrimento entre as funções de onda eletrônicas. Tanto o exchange intra-atômico quanto o exchange entre elétrons que possuem recobrimento entre suas funções de onda são classificados como diretos, pois não dependem de nenhuma intermediação. Outras formas de exchange, classificadas como indiretas podem ocorrer mesmo que as funções de onda dos elétrons interagentes não tenham recobrimento apreciável. Uma delas é o chamado superexchange, presente em compostos iônicos, como os óxidos metálicos, onde a interação entre os spins de dois átomos do metal é mediada pelo átomo não magnético situado entre os elementos metálicos [39]. Outros exemplos de exchange indireto são o exchange duplo, análogo ao superexchange para átomos com valências diferentes [40] e o exchange RKKY, que é a interação entre spin nucleares, mediada pela interação hiperfina com elétrons de condução, ou entre elétrons de dois íons, mediada pela interação de exchange entre elétrons de camadas internas e elétrons de condução [41].

\subsubsection{Hamiltoniano de Heisenberg}

Na seção anterior, foi mostrado que mesmo sem figurar no hamiltoniano do sistema, a orientação dos spins eletrônicos influencia diretamente o espectro de energias do sistema. Nesta seção, será encontrado um operador auxiliar que só depende de variáveis de spin e fornece exatamente o mesmo espectro de energias. A vantagem do operador fictício está em sua simplicidade, onde transparece a correlação entre a orientação relativa dos spins eletrônicos e a energia (coulombiana) do sistema.

No caso do átomo de He, por exemplo, foi visto que o estado singleto, $S=0$, onde os spins estão alinhados antiparalelamente tem energia maior do que a do tripleto, $S=1$, onde o alinhamento entre os spins é paralelo. Uma maneira de formular essa situação física é representar a energia de interação através do produto escalar entre operadores 
de spin, orientados pelo eixo de quantização dos spins individuais,

$$
\mathcal{H}_{X}=A s_{1} \cdot s_{2}
$$

onde a constante $A$ será determinada de forma a adequar esse operador ao problema. Dessa maneira a orientação entre os spins influencia na energia do sistema, pois se $s_{1}$ é antiparalelo a $s_{2}, E_{\uparrow \downarrow}=-A$ e se $s_{1}$ é paralelo a $s_{2}, E_{\uparrow \uparrow}=+A$.

Para que essa formulação seja válida, a função de onda total deve ser um autoestado do operador $S^{2}$, o qual que acopla os spins individuais segundo $S_{=} s_{1}+s_{2}$. O valor esperado de $S^{2}$, definido como $\left\langle S^{2}\right\rangle$ deve resultar nos autovalores $S(S+1)$, de forma que pode-se escrever

$$
\left\langle s_{1}^{2}+s_{2}^{2}+2 s_{1} \cdot s_{2}\right\rangle=S(S+1)
$$

O mesmo vale para os spins individuais, portanto

$$
\left\langle s_{i}^{2}\right\rangle=s(s+1)=3 / 4
$$

A substituição de (2.24) em (2.23) resulta em $\left\langle s_{1} . s_{2}\right\rangle=-3 / 4$ para o singleto $(S=0)$ e $\left\langle s_{1} \cdot s_{2}\right\rangle=1 / 4$ para o tripleto $(S=1)$, de forma que a diferença de energia entre esses dois estados pode ser calculada pelo valor esperado $\left\langle\mathcal{H}_{X}\right\rangle$, que resulta em $E_{\uparrow \downarrow}$ para o singleto e $E_{\uparrow \uparrow}$ para o tripleto

$$
\Delta E_{X}=E_{\downarrow \uparrow}-E_{\uparrow \uparrow}=A(-3 / 4-1 / 4)=-A
$$

Dessa maneira, com $A=-2 J$ é possível definir o hamiltoniano efetivo de interação entre dois spins como

$$
\mathcal{H}_{X}=-2 J s_{1} \cdot s_{2}
$$

onde a constante $J$ é determinada pela integral (2.21).

Essa formulação pode ser generalizada também para um sistema de muitos elétrons e escrita sob a forma conhecida como hamiltoniano de Heisenberg

$$
\mathcal{H}_{X}=-2 \sum_{i<j} J_{i j} \boldsymbol{S}_{i} \cdot \boldsymbol{S}_{j}
$$


onde $\boldsymbol{S}_{i}$ e $\boldsymbol{S}_{j}$ são os spins totais de dois átomos $i$ e $j$, e $J_{i, j}$ é um parâmetro (integral de exchange) que caracteriza a interação entre esses dois átomos.

A descrição da interação de exchange através do hamiltoniano de Heisenberg é uma aproximação, que pode ser entendida como o primeiro termo de uma expansão da energia de exchange em uma série de potências [40]. Nessa expansão o próximo termo de ordem superior tem a forma

$$
-\sum_{i \neq j} J_{i j}^{\prime}\left(S_{i} \cdot S_{j}\right)^{2}
$$

e é chamado de exchange biquadrático. Quando $J_{i j}^{\prime}<0$ o termo $\cos ^{2} \theta$, que determina a dependência da energia de exchange com o ângulo $\theta$ entre os spins, favorece um alinhamento perpendicular de spins.

O hamiltoniano de Heisenberg pode ser utilizado para caracterizar diversos sistemas físicos, tanto para o exchange intra-atômico como para as diferentes formas de exchange inter-atômico, direto ou indireto, fornecendo um ferramenta poderosa para a interpretação de fenômenos magnéticos em física da matéria condensada. 


\subsection{O modelo do campo efetivo para o magnetismo}

Na seção precedente foi apresentado o mecanismo de interação pelo qual os spins eletrônicos de um dado sistema podem organizar-se em orientações particulares. Especificamente para um estado excitado do átomo de hélio, foi demonstrado que o estado singleto de spin tem energia menor do que o tripleto de spin, sendo portanto mais estável. Isso significa que a interação de exchange favorece certos alinhamentos de spin em relação a outros. Esse mecanismo pode ser estendido também para moléculas, sólidos e outros sistemas materiais. Nesta seção será mostrado como essa a interação entre espécies magnéticas de um material pode originar alinhamentos particulares dos momentos magnéticos individuais que se estendem por toda a rede. Para tal será utilizada a teoria do campo efetivo, idealizada inicialmente por Pierre Weiss [42] no início do século XX e posteriormente aprofundada $[43,44]$ com a introdução da mecânica quântica de átomos e moléculas. Essa teoria consiste em aproximar o efeito de todas as interações sofridas por um dado sítio magnético, por uma única interação com um campo magnético efetivo. Embora muito simples, esse modelo é capaz de prever os principais tipos de arranjos magnético.

Em geral, pode-se distinguir entre três tipos de magnetismo: diamagnetismo, paramagnetismo e ferromagnetismo. O diamagnetismo e o paramagnetismo ocorrem quando não há interação de exchange entre as espécies magnéticas, enquanto que o ferromagnetismo não só depende, como origina-se da interação de exchange entre sítios magnéticos. Nos sistemas ferromagnéticos diversos estados ordenados são possíveis, entre eles o ferromagnético (FM), o antiferromagnético (AFM) e o ferrimagnético (FiM), os quais serão discutidos nesta seção. Uma maneira de classificar os sistemas magnéticos, é observar suas reações à aplicação de um campo magnético externo. Isso é feito determinando-se as grandezas magnetização, $M$, e susceptibilidade magnética, $\chi$, do material, as quais geralmente dependem de $B$ e $T$, e resumem grande parte da informação acerca da estrutura magnética de um dado sistema. Essas grandezas serão definidas a partir de variáveis termodinâmicas, e calculadas para os principais sistemas magnéticos conhecidos, através do formalismo mecânico quântico de átomos e 
moléculas.

Primeiramente será analisado o efeito do campo sobre um elétron livre e sobre um átomo isolado. Em seguida serão incluídos moléculas e sólidos com espécies magnéticas não interagentes e finalmente será analisado um sólido onde os sítios magnéticos interagem por exchange. Para demonstrar como a interação de exchange pode levar um os de átomos de um material a acomodarem seus momentos magnéticos em estruturas ordenadas, será utilizada a aproximação do campo médio de Weiss, a qual embora muito simples, é capaz de descrever satisfatoriamente diversos sistemas magnéticos. Nessa aproximação a soma de todas as interações de um dado átomo é parametrizada em um campo médio, o campo de Weiss, que atua nesse átomo da mesma maneira que um campo magnético o faz. Para isso é necessário conhecer inicialmente o efeito da aplicação de um campo magnético sobre um ou mais átomos, analisando o efeito disso nos níveis de energia e na orientação dos momentos magnéticos envolvidos.

\subsubsection{Partículas carregadas num campo magnético uniforme}

Seja uma partícula de massa $m$ e carga - $e$ sujeita a um campo magnético uniforme. Esse campo magnético introduz sobre a partícula uma força de Lorentz

$$
\boldsymbol{F}=-e \boldsymbol{v} \times \boldsymbol{B}
$$

onde $\boldsymbol{v}$ é a velocidade da partícula e $\boldsymbol{B}$ é o vetor campo magnético. A força de Lorentz magnética não realiza trabalho, pois $\boldsymbol{F} \cdot \boldsymbol{v}=0$, de forma que a energia dessa partícula é puramente cinética, dada por

$$
\mathcal{H}=\frac{1}{2} m v^{2}
$$

Essa hamiltoniana clássica não é capaz de descrever o magnetismo na matéria, de acordo com o teorema de Bohr-van Leeuwen [45], de forma que faz-se necessário obter o hamiltoniano quântico dessa partícula. Isso pode ser feito a partir da hamiltoniana clássica, desde que a mesma seja formulada em termos das variáveis canônicas do 
sistema, isto é, das variáveis que obedeçam às equações de Hamilton

$$
\begin{aligned}
\frac{d}{d t} r_{i} & =\frac{\partial \mathcal{H}}{\partial p_{i}} \\
-\frac{d}{d t} p_{i} & =\frac{\partial \mathcal{H}}{\partial r_{i}}
\end{aligned}
$$

onde $i$ representa uma das coordenadas espaciais $x, y$ ou $z$ [46].

A seguir, as variáveis canônicas desse sistema serão determinadas pelo procedimento usual e a hamiltoniana clássica será descrita em termos do vetor momento generalizado, o qual posteriormente será substituído pelo operador momento para a construção do hamiltoniano quântico.

Inicialmente, deve-se observar que a inexistência de pólos magnéticos individuais impõe ao campo magnético a restrição

$$
\nabla \cdot B=0
$$

a qual faz parte das equações de Maxwell do magnetismo. Uma forma de garantir que o divergente de $\boldsymbol{B}$ sempre se anule, é lançar mão da identidade vetorial $\boldsymbol{\nabla} \cdot(\boldsymbol{\nabla} \times \boldsymbol{x})=0$, onde $\boldsymbol{x}$ é um vetor qualquer, e definir o campo magnético em termos do rotacional de um vetor auxiliar, o potencial vetor, $\boldsymbol{A}$.

$$
B(r)=\nabla \times A(r)
$$

Com essa descrição a força resultante sobre a partícula é

$$
F=-e v \times \nabla \times A
$$

de forma que pela segunda lei de Newton,

$$
m \frac{d \boldsymbol{v}}{d t}=-e \boldsymbol{v} \times \nabla \times \boldsymbol{A}
$$

Utilizando a identidade vetorial $v \times(\nabla \times A)=\nabla(v \cdot A)-(v \cdot \nabla) A$, a equação (2.36) pode ser reescrita como

$$
m \frac{d \boldsymbol{v}}{d t}=-e \nabla(\boldsymbol{v} \cdot \boldsymbol{A})-e(\boldsymbol{v} \cdot \boldsymbol{\nabla}) \boldsymbol{A}
$$


O termo $(\boldsymbol{v} \cdot \boldsymbol{\nabla}) \boldsymbol{A}$ está relacionado com o derivativo conectivo de $\boldsymbol{A}$, o qual descreve a derivada temporal de um campo vetorial em um sistema de coordenadas que se move com velocidade $\boldsymbol{v}$, neste caso o referencial da partícula. De acordo com a referência [47], esse derivativo é dado por

$$
\frac{d \boldsymbol{A}}{d t}=\frac{\partial \boldsymbol{A}}{\partial t}+(\boldsymbol{v} \cdot \nabla) \boldsymbol{A}
$$

Como o campo magnético é uniforme no tempo e no espaço, $\frac{\partial \boldsymbol{A}}{\partial t}=0$, de forma que a equação (2.37) pode ser reescrita como

$$
\frac{d}{d t}(m \boldsymbol{v}-e \boldsymbol{A})=-e \nabla(\boldsymbol{v} \cdot \boldsymbol{A})
$$

Assim pode-se definir o momento generalizado $\boldsymbol{p}=m \boldsymbol{v}-e \boldsymbol{A}$, o qual juntamente com a variável canônica conjugada, neste caso o vetor posição $r$, obedece às equações de Hamilton (2.31) e (2.32). Em termos das variáveis canônicas a hamiltoniana da partícula é dada por

$$
\mathcal{H}=\frac{1}{2 m}(\boldsymbol{p}+e \boldsymbol{A})^{2}=\frac{1}{2 m}\left(\boldsymbol{p}^{2}+e \boldsymbol{p} \cdot \boldsymbol{A}+e \boldsymbol{A} \cdot \boldsymbol{p}+e^{2} \boldsymbol{A}^{2}\right)
$$

O potencial vetor $\boldsymbol{A}$ pode ser definido arbitrariamente, desde que obedeça a relação (2.34). Uma escolha possível é

$$
A(r)=\frac{1}{2}(B \times r)
$$

como pode ser verificado diretamente por substituição em (2.34). Esse potencial vetor tem o divergente nulo, de forma que $\boldsymbol{p} \cdot \boldsymbol{A}=\boldsymbol{A} \cdot \boldsymbol{p}=\frac{1}{2} \boldsymbol{r} \times \boldsymbol{p} \cdot \boldsymbol{B}$. Considerando isso, a substituição de (2.41) em (2.40) resulta em

$$
\mathcal{H}(\boldsymbol{r}, \boldsymbol{p})=\frac{1}{2 m}\left(\boldsymbol{p}^{2}+e \boldsymbol{r} \times \boldsymbol{p} \cdot \boldsymbol{B}+\frac{e^{2}}{4}(\boldsymbol{B} \times \boldsymbol{r})^{2}\right)
$$

A hamiltoniana de uma partícula carregada em um campo magnético uniforme pode ser estendida para descrever um ou mais elétrons em um átomo, incluindo em (2.42) um potencial nuclear $V_{i}$ para cada elétron e um termo do efeito do campo magnético sobre o núcleo, o qual pode ser eliminado escrevendo-se a hamiltoniana no referencial 
do núcleo, que com boa aproximação coincide com o referencial do centro de massa. Nesse referencial a hamiltoniana de um átomo de $i$ elétrons é dada por

$$
\mathcal{H}\left(\boldsymbol{r}_{i}, \boldsymbol{p}_{i}\right)=\sum_{i}\left[\frac{1}{2 m}\left(\boldsymbol{p}_{i}^{2}+e \boldsymbol{r} \times \boldsymbol{p}_{i} \cdot \boldsymbol{B}+\frac{e^{2}}{4}\left(\boldsymbol{B} \times \boldsymbol{r}_{i}\right)^{2}\right)+V_{i}\right]
$$

É interessante notar que o termo $\boldsymbol{r} \times \boldsymbol{p} \cdot \boldsymbol{B}$ pode ser reescrito como $\boldsymbol{r} \times\left(m \boldsymbol{v}+\frac{e}{2} \boldsymbol{B} \times \boldsymbol{r}\right)$. $\boldsymbol{B}=\boldsymbol{r} \times m \boldsymbol{v} \cdot \boldsymbol{B}$, aonde pode-se identificar o momento angular $\boldsymbol{r} \times m \boldsymbol{v}$ de cada elétron, de forma que a soma em $i$ corresponde ao momento angular orbital do átomo, $\boldsymbol{L}$. Dessa maneira foi possível relacionar o efeito do campo magnético com o momento angular orbital do átomo. A hamiltoniana em termos de $\boldsymbol{L}$ fica dada por

$$
\mathcal{H}\left(\boldsymbol{r}_{i}, \boldsymbol{p}_{i}\right)=\sum_{i}\left[\frac{1}{2 m}\left(\boldsymbol{p}_{i}^{2}+e \boldsymbol{L} \cdot \boldsymbol{B}+\frac{e^{2}}{4}\left(\boldsymbol{B} \times \boldsymbol{r}_{i}\right)^{2}\right)+V_{i}\right]
$$

A hamiltoniana (2.44) pode ser utilizada para a construção do operador hamiltoniano do sistema seguindo-se a prescrição onde o vetor momento generalisado é substituído pelo operador momento. Além disso, deve-se incluir o grau de liberdade de spin dos elétrons, o qual não está previsto classicamente. Para isso dois termos devem ser somados ao hamiltoniano. O primeiro é referente à interação Zeeman do spin atômico com o campo magnético

$$
\boldsymbol{\mu} \cdot \boldsymbol{B}=-g_{0} \mu_{b} \frac{\boldsymbol{S}}{\hbar} \cdot \boldsymbol{B}
$$

onde $\mu_{B}=e \hbar / 2 m=9.274 \times 10^{-24} \mathrm{~J} / \mathrm{T}$ é o magneton de Bohr. O segundo termo é referente à interação spin-órbita entre os momentos angulares orbital e de spin do átomo

$$
\lambda_{\mathrm{SO}} \boldsymbol{L} \cdot \boldsymbol{S}
$$

onde $\lambda_{\mathrm{SO}}$ é a constante de acoplamento.

A inclusão desses dois termos na hamiltoniana (2.44) e a substituição de $p$ por $-\imath \hbar \nabla$ resultam no operador hamiltoniano

$$
\mathcal{H}=\sum_{i}\left(-\frac{\hbar^{2}}{2 m} \nabla^{2}+V_{i}\right)+\frac{e}{2 m} \boldsymbol{L} \cdot \boldsymbol{B}+\sum_{i} \frac{e^{2}}{8 m}\left(\boldsymbol{B} \times \boldsymbol{r}_{i}\right)^{2}+g_{0} \mu_{B} \frac{\boldsymbol{S}}{\hbar} \cdot \boldsymbol{B}+\lambda_{\mathrm{SO}} \boldsymbol{L} \cdot \boldsymbol{S}
$$


O primeiro termo de (2.47) corresponde ao hamiltoniano de um átomo livre e será denominado $\mathcal{H}_{0}$. O termo spin-órbita acopla os momentos angulares orbital e de spin num momento angular total, $\boldsymbol{J}$, que é definido por

$$
\boldsymbol{J}=\boldsymbol{L}+\boldsymbol{S}
$$

As grandezas $\boldsymbol{L}$ e $\boldsymbol{S}$ não são conservadas individualmente, mas o momento angular total $\boldsymbol{J}$ é conservado, de forma que o hamiltoniano do sistema deve ser descrito em termos de $\boldsymbol{J}$. Os termos da interação de $\boldsymbol{L}$ e $\boldsymbol{S}$ com o campo magnético podem ser agrupados utilizando-se a aproximação

$$
\frac{\mu_{B}}{\hbar}\left(\boldsymbol{L}+g_{0} \boldsymbol{S}\right) \simeq \frac{\mu_{B}}{\hbar} \frac{\boldsymbol{L} \cdot \boldsymbol{J}+g_{0} \boldsymbol{S} \cdot \boldsymbol{J}}{J^{2}} \boldsymbol{J}=\frac{\mu_{B}}{2 \hbar} \frac{\boldsymbol{J}^{2}+\boldsymbol{L}^{2}-\boldsymbol{S}^{2}+g_{0}\left(\boldsymbol{J}^{2}-\boldsymbol{L}^{2}+\boldsymbol{S}^{2}\right)}{J^{2}} \boldsymbol{J}
$$

válida quando o termo spin-órbita é dominante [37]. Como o valor médio de um operador momento angular ao quadrado é dado por

$$
\left\langle J^{2}\right\rangle=J(J+1)
$$

ao substituirmos os operadores de momento angular $\boldsymbol{L}, \boldsymbol{S}$ e $\boldsymbol{J}$ em (2.49) pelos seus respectivos autovalores, obtemos

$$
\begin{array}{r}
\frac{\mu_{B}}{\hbar}\left(\boldsymbol{L}+g_{0} \boldsymbol{S}\right)= \\
\hline(J+1)+L(L+1)-S(S+1)+g_{0}(J(J+1)-L(L+1)+S(S+1)) \\
\hline 2 J(J+1)
\end{array}
$$

onde $g$ é denominado fator-g de Landé.

A substituição de $\mathcal{H}_{0}$ e (2.51) em (2.47) resulta no hamiltoniano total de um átomo de $i$ elétrons na presença de um campo magnético

$$
\mathcal{H}=\mathcal{H}_{0}+g \mu_{B} \boldsymbol{J} \cdot \boldsymbol{B}+\sum_{i} \frac{e^{2}}{8 m}\left(\boldsymbol{B} \times \boldsymbol{r}_{i}\right)^{2}+\lambda_{\mathrm{SO}} \boldsymbol{L} \cdot \boldsymbol{S}
$$

A partir do hamiltoniano (2.52) será possível determinar as características magnéticas do sistema resolvendo-se a equação de Schrödinger por teoria de perturbação até segunda ordem. Contudo, antes de prosseguir com a solução da equação de Schrödinger, podemos notar que o formalismo utilizado na descrição do magnetismo de 
um átomo isolado pode ser generalizado para um conjunto de átomos idênticos de momento angular total $\boldsymbol{J}$ que não interagem magneticamente.

O hamiltoniano (2.52) de um átomo pode ser utilizado para descrever todos os átomos, uma vez que todos são idênticos. O comportamento de cada átomo na presença do campo implicará num comportamento geral do material, que é caracterizado em termos da magnetização total, definida como o momento magnético por unidade de volume do material. Num material de volume $V$, com uma densidade $N$ de sítios magnéticos de momento magnético $\boldsymbol{\mu}$, a magnetização total é definida como

$$
M=\frac{1}{V} \sum_{i=1}^{N V} \boldsymbol{\mu}_{i}
$$

A aplicação de um campo magnético a esse material pode alterar a direção dos momentos magnéticos de cada átomo, o que influencia diretamente a magnetização total. A variação da magnetização devida ao campo magnético externo é quantificada em termos da grandeza susceptibilidade magnética, $\chi$, definida de maneira geral como

$$
\chi_{v}=\frac{\partial M_{v}}{\partial B_{v}}
$$

onde $v$ representa cada uma das componentes. Se $\boldsymbol{M}$ depende linearmente de $\boldsymbol{B}$,

$$
\boldsymbol{M}=\chi \boldsymbol{B}
$$

Tanto a magnetização quanto a susceptibilidade em geral podem depender da temperatura. Para determinar o efeito da temperatura, algumas considerações termodinâmicas devem ser feitas. Vamos considerar um sistema de $N / V$ spins $S$ não interagentes, o qual em $T=0 \mathrm{~K}$ tem todos os momentos magnéticos orientados aleatoriamente. Nesse estado, de energia $E_{0}$, os momentos magnéticos individuais cancelam-se e a magnetização média é nula. Em temperaturas finitas as flutuações térmicas podem popular estados excitados de energia $E_{n}$, com uma probabilidade

$$
P=\frac{\exp ^{-\frac{E_{n}}{k T}}}{Z}
$$

onde $k=1.38 \times 10^{-23} \mathrm{~J} / \mathrm{K}$ é a constante de Boltzmann e

$$
Z=\sum_{n} e^{-\frac{E_{n}}{k T}}
$$


é a função de partição do sistema. No equilíbrio térmico, a magnetização é a média das magnetizações dos estados acessíveis, ponderada pela probabilidade de ocorrência de cada estado

$$
M(B, T)=\frac{1}{Z} \sum_{n} M_{n}(B) e^{-\frac{E_{n}}{k T}}
$$

A magnetização de cada átomo, é determinada por seu momento magnético

$$
\boldsymbol{\mu}=-g \mu_{B} \boldsymbol{J}
$$

Supondo o campo magnético orientado na direção $z$, a energia de cada estado $n$ é dada por

$$
E_{m}=-g \mu_{B} m_{J} B
$$

onde $m_{J}$ são os autovalores de $J_{z}$, que podem assumir os valores $m_{J}=-J,-J+1, \ldots, J$. Dessa maneira a magnetização dos $N$ átomos é dada por

$$
M(B, T)=N g \mu_{B} \frac{1}{Z} \sum_{J} m_{J} e^{-\frac{g \mu_{B} B}{k T} m_{J}}=N g \mu_{B}\left\langle J_{z}\right\rangle
$$

A soma $\sum_{J} m_{J} e^{\frac{-g \mu_{B} B}{k T} m_{J}}$ equivale a $\frac{\partial Z}{\partial\left(\frac{g \mu_{B} B}{k T}\right)}$ e a magnetização (2.61) pode ser escrita como

$$
M(B, T)=-N g \mu_{B} \frac{1}{Z} \frac{\partial Z}{\partial\left(\frac{g \mu_{B} B}{k T}\right)}
$$

A diferenciação em relação a $\frac{g \mu_{B} B}{k T}$ pode ser escrita em termos de $B$

$$
\frac{\partial Z}{\partial\left(\frac{g \mu_{B} B}{k T}\right)}=\frac{\partial Z}{\partial B} \frac{\partial B}{\partial\left(\frac{g \mu_{B} B}{k T}\right)}=\frac{\partial Z}{\partial B} \frac{k T}{g \mu_{B}}
$$

de forma que

$$
M(B, T)=N k T \frac{1}{Z} \frac{\partial Z}{\partial B}
$$

Usando a relação $\frac{d \ln u}{d x}=\frac{1}{u} \frac{d u}{d x}$,

$$
M(B, T)=N k T \frac{\partial \ln Z}{\partial B}
$$


Essa equação pode ser escrita em função da energia livre de Helmholtz

$$
F=-k T \ln Z
$$

resultando na definição termodinâmica da magnetização

$$
M(B, T)=-N\left(\frac{\partial F}{\partial B}\right)_{V, T}
$$

a qual permite relacionar a magnetização de um material com a variação da energia interna devida a $B$. Diferenciando-se (2.67) em relação a $B$, pode-se obter a susceptibilidade em termos de $F$

$$
\chi=-N \frac{\partial^{2} F}{\partial B^{2}}
$$

Agora que as grandezas magnetização e susceptibilidade de um sistema multiatômico foram definidas, já é possível prosseguir calculando as correções da energia introduzidas pela aplicação do campo e determinar as magnetizações e susceptibilidades a partir de (2.67) e (2.68).

Os estados estacionários do sistema são dados pela solução da equação de Schrödinger

$$
\mathcal{H} \Psi_{n}=E_{n} \Psi_{n}
$$

onde $\Psi_{n}$ são os autoestados do hamiltoniano de correspondentes autovalores $E_{n}$.

O hamiltoniano $\mathcal{H}_{0}$ tem solução analítica no modelo do átomo hidrogenoide. Por isso o hamiltoniano (2.52) será separado em dois termos, $\mathcal{H}_{0}+\mathcal{H}_{1}$. A energia total do sistema é obtida resolvendo-se analiticamente a equação de Schrödinger para $\mathcal{H}_{0}$, que fornece a energia $E_{0}$, a qual deve então ser corrigida pela energia da perturbação $\mathcal{H}_{1}$.

No estado fundamental $\psi_{0}$, o autovalor de $\mathcal{H}_{0}$ é dado por

$$
E_{0}=\int \psi_{0}^{*} \mathcal{H}_{0} \psi_{0} d \tau
$$

e pode ser obtido utilizando-se funções de onda hidrogenoides. Os termos spin-órbita e de interação com o campo são tratados como perturbações e de acordo com [39] a 
correção na energia até segunda ordem é dada por

$$
\Delta E_{0}=\int \psi_{n}^{*} \mathcal{H}_{\text {pert }} \psi_{n} d \tau+\sum_{m \neq n} \frac{\left|\int \psi_{n}^{*} \mathcal{H}_{\text {pert }} \psi_{m} d \tau\right|^{2}}{E_{n}-E_{m}}
$$

Da definição de $\boldsymbol{J}$ apresentada na equação (2.48), o hamiltoniano da perturbação devido ao acoplamento spin-órbita $\mathcal{H}_{\mathrm{SO}}=\lambda_{\mathrm{SO}} \boldsymbol{L} \cdot \boldsymbol{S}$ pode ser re-escrito na forma

$$
\mathcal{H}_{\mathrm{SO}}=\lambda_{\mathrm{SO}} \frac{\boldsymbol{J}^{2}-\boldsymbol{L}^{2}-\boldsymbol{S}^{2}}{2}
$$

de forma que a correção na energia devida a esse termo, até primeira ordem, da equação (2.71) é dada por

$$
\Delta E_{\mathrm{SO}}=\left\langle\psi_{0}^{*}\left|\lambda_{\mathrm{SO}} \frac{\boldsymbol{J}^{2}-\boldsymbol{L}^{2}-\boldsymbol{S}^{2}}{2}\right| \psi_{0}\right\rangle
$$

o que considerando a equação (2.50) resulta em

$$
\Delta E_{\mathrm{SO}}=\frac{\lambda_{\mathrm{SO}}}{2}[J(J+1)-L(L+1)-S(S+1)]
$$

O efeito do campo externo na energia do sistema manifesta-se no hamiltoniano total (2.52) com dois termos que dependem de $\boldsymbol{B}$. Cada um desses termos é responsável por um tipo de magnetismo: o termo linear em $\boldsymbol{B}$ é responsável pelo paramagnetismo e o termo quadrático em $\boldsymbol{B}$ é responsável pelo diamagnetismo. A seguir iremos calcular separadamente as correções na energia $\Delta E$ para os casos em que predomina o termo diamagnético ou o termo paramagnético.

\section{Diamagnetismo}

Quando o material não possui elétrons desemparelhados, os momentos angulares totais orbital e de spin são nulos. Nesse caso o termo paramagnético em (2.52) é anulado, de forma que a resposta do material à aplicação do campo magnético é exclusivamente diamagnética. Por isso, mesmo estando o diamagnetismo presente em todos os materiais, chama-se de diamagnético o material que não possui elétrons desemparelhados. 
Para o termo diamagnético temos

$$
\mathcal{H}_{\mathrm{D}}=\frac{e^{2}}{8 m}(\boldsymbol{B} \times \boldsymbol{r})^{2}
$$

a correção perturbativa até primeira ordem é dada por

$$
\Delta E_{0}=\int \psi_{0}^{*} \frac{e^{2}}{8 m}(\boldsymbol{B} \times \boldsymbol{r})^{2} \psi_{0} d \tau
$$

Para um campo magnético orientado na direção $z$, tem-se

$$
(\boldsymbol{B} \times \boldsymbol{r})^{2}=B^{2}\left(x^{2}+y^{2}\right)
$$

de forma que

$$
\Delta E_{\mathrm{D}}=\frac{e^{2} B^{2}}{8 m} \int \psi_{0}^{*}\left(x^{2}+y^{2}\right) \psi_{0} d \tau
$$

Como o momento angular é nulo para que o termo diamagnético seja predominante, as funções de onda eletrônicas são esfericamente simétricas

$$
\int \psi_{0}^{*} x^{2} \psi_{0} d \tau=\int \psi_{0}^{*} y^{2} \psi_{0} d \tau=\frac{1}{3} \int \psi_{0}^{*} r^{2} \psi_{0} d \tau
$$

e portanto

$$
\Delta E_{\mathrm{D}}=\frac{e^{2} B^{2}}{12 m} \int \psi_{0}^{*} r^{2} \psi_{0} d \tau=\frac{e^{2} B^{2}}{12 m}\left\langle r^{2}\right\rangle
$$

Considerando-se que no equilíbrio térmico os átomos encontram-se no estado fundamental $\psi_{0}$, a energia livre de Helmholtz para esse estado é dada por $F=E_{0}+\Delta E_{\mathrm{D}}$, de forma que a magnetização (2.67) fica

$$
M=-N \frac{\partial F}{\partial B}=-\frac{e^{2}}{6 m} B \sum_{i}\left\langle r^{2}\right\rangle
$$

e a susceptibilidade diamagnética vale

$$
\chi_{D}=-N \frac{e^{2}}{6 m} \sum_{i}\left\langle r_{i}^{2}\right\rangle
$$

Esse resultado permite concluir que $\chi_{D}$ é sempre negativa, o que significa que uma magnetização é gerada no sentido contrário ao do campo aplicado e nessa aproximação, não depende da temperatura. 


\section{Paramagnetismo}

Os materiais constituídos por átomos com elétrons desemparelhados, geralmente possuem um momento angular total, e por conseguinte, um momento magnético não nulos. Nos casos em que a interação entre esses momentos magnéticos pode ser desprezada, o sistema é descrito pelo hamiltoniano (2.52). Agora o termo predominante é o paramagnético (PM), e o termo diamagnético será desprezado. Para determinar a magnetização do paramagneto será calculada a correção perturbativa com o termo $\boldsymbol{\mu} \cdot \boldsymbol{B}$ de onde será demonstrado que os momentos magnéticos atômicos alinham-se ao sentido do campo magnético. Porém existe um efeito térmico que tende a desmanchar esse alinhamento. Flutuações térmicas populam estados excitados aleatoriamente, causando mudanças nos momentos magnéticos atômicos. Quanto maior a temperatura, mais difícil é magnetizar-se o material, o que leva à conclusão de que a susceptibilidade paramagnética deve ser inversamente proporcional à temperatura $\chi \propto \frac{1}{T}$. Essa expressão é conhecida como lei de Curie, e fora proposta no final do século XIX pelo físico francês Pierre Curie.

Seguindo a mesma abordagem do caso diamagnético, deseja-se obter a correção perturbativa $\Delta E_{\mathrm{P}}$ devida à presença do campo magnético, a qual de acordo com (2.71) é dada até segunda ordem por

$$
\Delta E_{\mathrm{P}}=\int \psi_{n}^{*} g \mu_{B} \boldsymbol{J} \cdot \boldsymbol{B} \psi_{n} d \tau+\sum_{m \neq n} \frac{\left|\int \psi_{n}^{*} g \mu_{B} \boldsymbol{J} \cdot \boldsymbol{B} \psi_{m} d \tau\right|^{2}}{E_{n}-E_{m}}
$$

Para um campo orientado na direção $z$, a correção da energia até a primeira ordem é

$$
\Delta E_{\mathrm{P}}=\int \psi_{n}^{*} g \mu_{B} J_{z} B \psi_{n} d \tau=g \mu_{B} J_{z} B
$$

onde $J_{z}$ é a projeção de $\boldsymbol{J}$ no eixo $z$, a qual pode assumir os valores $J_{z}=-J,-J+1, \ldots, J$. A função de partição para uma energia $\Delta E_{\mathrm{P}}=E_{J_{z}}(B)$ é dada por

$$
Z=\sum_{m=-J}^{J} \exp \left(\frac{-E_{J_{z}}(B)}{k T}\right)=\sum_{m=-J}^{J} \exp \left(\frac{-g \mu_{B} J_{z} B}{k T}\right)
$$


Essa função de partição pode ser manipulada para (cf. pg. 15 da referência [48], por exemplo)

$$
Z=\frac{\operatorname{senh}\left(\frac{2 J+1}{2 J} \frac{g \mu_{B} J B}{k T}\right)}{\operatorname{senh}\left(\frac{1}{2 J} \frac{g \mu_{B} J B}{k T}\right)}
$$

Para determinar a magnetização desse sistema, dada pela equação (2.64)

$$
M(B, T)=N k T \frac{1}{Z} \frac{\partial Z}{\partial B}
$$

é necessário efetuar a derivação de $Z$ em relação a $B$. Sabendo que

$$
\frac{d}{d x} \operatorname{senh}(a x)=a \cosh (a x)
$$

podemos escrever

$$
\frac{\partial Z}{\partial B}=\frac{\frac{(2 J+1) g \mu_{B} J}{2 J k T} \cosh \left(\frac{(2 J+1) g \mu_{B} J}{2 J k T} B\right) \operatorname{senh}\left(\frac{g \mu_{B} J}{2 J k T} B\right)-\frac{g \mu_{B} J}{2 J k T} \operatorname{senh}\left(\frac{(2 J+1) g \mu_{B} J}{2 J k T} B\right) \cosh \left(\frac{g \mu_{B} J}{2 J k T} B\right)}{\operatorname{senh}^{2}\left(\frac{g \mu_{B} J}{2 J k T} B\right)}
$$

Dividindo-se por Z, obtemos

$$
\frac{1}{Z} \frac{\partial Z}{\partial B}=\frac{g \mu_{B} J}{k T}\left[\frac{2 J+1}{2 J} \operatorname{coth}\left(\frac{2 J+1}{2 J} \frac{g \mu_{B} J}{k T} B\right)-\frac{1}{2 J} \operatorname{coth}\left(\frac{1}{2 J} \frac{g \mu_{B} J}{k T} B\right)\right]
$$

Esse resultado pode ser exprimido em termos da função de Brillouin, definida como

$$
\mathcal{B}_{J}(y)=\frac{2 J+1}{2 J} \operatorname{coth}\left(\frac{2 J+1}{2 J} y\right)-\frac{1}{2 J} \operatorname{coth}\left(\frac{y}{2 J}\right)
$$

dessa forma a magnetização

$$
M=N k T \frac{1}{Z} \frac{\partial Z}{\partial B}=N g \mu_{B} J\left[\frac{2 J+1}{2 J} \operatorname{coth}\left(\frac{2 J+1}{2 J} \frac{g \mu_{B} J}{k T} B\right)-\frac{1}{2 J} \operatorname{coth}\left(\frac{1}{2 J} \frac{g \mu_{B} J}{k T} B\right)\right]
$$

pode ser reduzida para a forma

$$
M=M_{S} \mathcal{B}_{J}\left(\frac{g \mu_{B} J}{k T} B\right)
$$

onde $M_{S}=N g \mu_{B} J$ é a magnetização de saturação do material, a magnetização na situação em que os momentos magnéticos encontram-se totalmente alinhados ao mesmo sentido. A figura (2.1) ilustra esse resultado para diversos valores de $J$, inclusive 


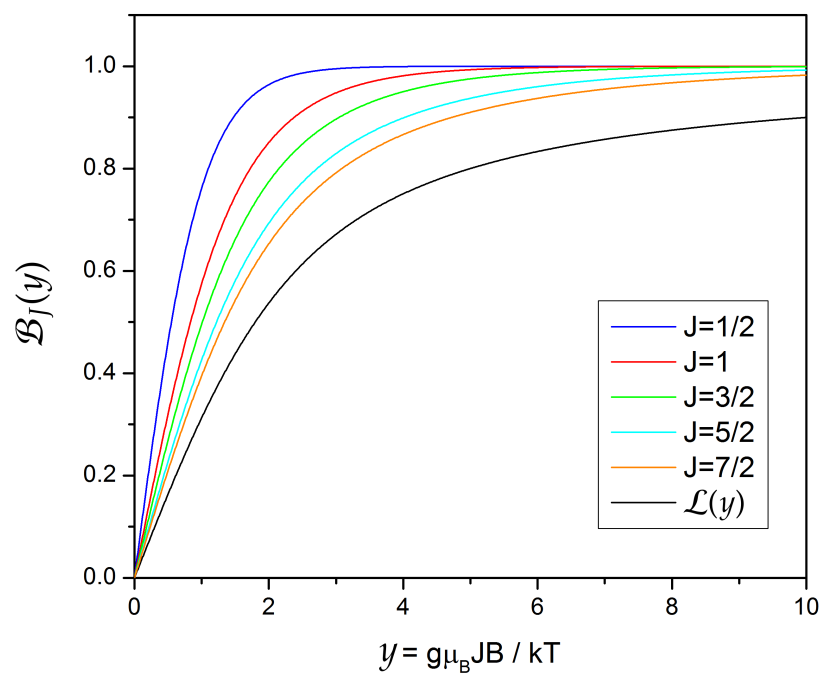

Figura 2.1: Função de Brillouin para diversos valores de J.

o limite clássico $J \rightarrow \infty$, onde a função de Brillouin se reduz à função de Langevin $\mathcal{L}(y)=\operatorname{coth}(y)-y^{-1}$.

A expansão em série de potências da função de Brillouin resulta em

$$
\mathcal{B}_{J}(y)=\frac{(J+1) y}{3 J}+O\left(y^{3}\right)+O\left(y^{5}\right)+\ldots
$$

de forma que em temperaturas altas, quando $y \ll 1$, os termos de potências superiores a 1 podem ser desprezados e a magnetização fica

$$
M=M_{S} \frac{J(J+1) g \mu_{B}}{3 J k T} B_{0}
$$

o que resulta para a susceptibilidade

$$
\chi=\frac{M_{S} J(J+1) g \mu_{B}}{3 J k_{B} T}
$$

Agrupando as constantes, define-se a constante de Curie

$$
C=\frac{(J+1) g \mu_{B} M_{S}}{3 k}
$$

o que permite chegar-se na Lei de Curie

$$
\chi=\frac{C}{T}
$$


O diamagnetismo e paramagnetismo com suas magnetizações e susceptibilidades características foram derivados a partir do efeito de um campo magnético sobre átomos isolados, ou num material onde as interações magnéticas são desprezíveis. Quando a interação entre as espécies magnéticas não é desprezível, o hamiltoniano (2.52) deve ser acrescido dos termos apropriados a essas interações. Na aproximação de Weiss, as interações de um dado sítio com os demais sítios da rede são agrupadas no campo médio, ou campo molecular, $B_{m}$, que atua em cada spin da rede exatamente como um campo magnético, de forma que no hamiltoniano total basta adicionar o campo $B_{m}$ ao campo magnético e proceder da mesma maneira que no caso paramagnético. A seguir, será demonstrado como essa simples formulação origina os principais tipos de magnetismo encontrados nos materiais.

\subsection{2 Átomos num campo magnético efetivo}

O campo efetivo em um sítio magnético é dado pelo campo magnético externo adicionado ao campo molecular $B_{m}$. No espírito do hamiltoniano de Heisenberg, a interação entre dois spins favorece um alinhamento dos mesmos, o qual pode ser paralelo ou antiparalelo, dependendo da natureza da interação de exchange, que pode ser positiva ou negativa. O campo molecular deve refletir essa interação e a maneira proposta por Weiss em sua teoria do campo efetivo é postular que o campo molecular seja proporcional à magnetização da rede

$$
\boldsymbol{B}_{m}=\lambda M
$$

onde a constante de proporcionalidade $\lambda$ deve ser determinada caso o caso.

No formalismo do hamiltoniano de Heisenberg (ver equação (2.27)) o i-ésimo sítio de spin $\boldsymbol{S}_{i}$, interage por exchange com todos os j-ésimos sítios vizinhos, de spin $\boldsymbol{S}_{j}$, segundo o hamiltoniano

$$
\mathcal{H}_{i}=-2 \sum_{j} J_{i j} \boldsymbol{S}_{i} \cdot \boldsymbol{S}_{j}
$$

onde $J_{i j}$ é a integral de exchange, uma constante que depende das funções de onda dos elétrons interagentes. Conforme proposto por Weiss, esse interação é equivalente 
a uma interação do tipo Zeeman entre o $i$-ésimo sítio e um campo molecular $\boldsymbol{B}_{m}$. O hamiltoniano dessa interação é dado por

$$
\mathcal{H}_{i}=-g \mu_{B} \boldsymbol{S}_{i} \cdot \boldsymbol{B}_{m}
$$

onde o sinal do lado direito é tomado como negativo, para que a interação de exchange favoreça o alinhamento dos spins quando $J_{i j}>0$.

Igualando-se as expressões (2.100) e (2.101), obtemos para o campo molecular atuante em um sítio $i$

$$
\boldsymbol{B}_{m}=\frac{2}{g \mu_{B}} \sum_{j} J_{i j} \boldsymbol{S}_{j}
$$

A aproximação de Weiss consiste em substituir cada spin $\boldsymbol{S}_{j}$ por um valor médio $\left\langle\boldsymbol{S}_{j}\right\rangle$, paralelo à magnetização do material, de forma que

$$
\boldsymbol{B}_{m}=\frac{2}{g \mu_{B}} \sum_{j} J_{i j}\left\langle\boldsymbol{S}_{j}\right\rangle
$$

Dado que as integrais de exchange diminuem com a distância entre os spins, podemos nos restringir à aproximação de primeiros vizinhos, a qual consiste em limitar o somatório em (2.103) apenas aos sítios mais próximos. Admitindo z primeiros vizinhos equivalentes, cuja interação de exchange é descrita por $J_{x c}$, obtemos

$$
\boldsymbol{B}_{m}=\frac{2}{g \mu_{B}} z J_{x c}\left\langle\boldsymbol{S}_{j}\right\rangle
$$

Dado que todos os átomos magnéticos são idênticos e equivalentes, o valor médio $\left\langle\boldsymbol{S}_{j}\right\rangle$ está relacionado com a magnetização da rede através da relação

$$
\boldsymbol{M}=N g \mu_{B}\left\langle\boldsymbol{S}_{j}\right\rangle
$$

o que substituído em (2.104) resulta em

$$
\boldsymbol{B}_{m}=\frac{2 z J_{x c}}{N g^{2} \mu_{B}^{2}} \boldsymbol{M}
$$

de onde, por comparação com (2.99), obtemos a constante $\lambda$ como

$$
\lambda=\frac{2 z J_{x c}}{N g^{2} \mu_{B}^{2}}
$$


O hamiltoniano do $i$-ésimo átomo de um material magnético na presença de um campo efetivo $\boldsymbol{B}=\boldsymbol{B}_{0}+\boldsymbol{B}_{m}$, onde $\boldsymbol{B}_{0}$ é o campo externo, é dado pela equação (2.52). Admitindo que a magnetização seja orientada na direção do campo, definida como a direção $z$, o produto escalar $\boldsymbol{J} \cdot\left(\boldsymbol{B}_{0}+\boldsymbol{B}_{m}\right)$ resulta em $J_{z}\left(B_{0}+B_{m}\right)$, o que torna o problema escalar, com o hamiltoniano de interação dado por

$$
\mathcal{H}=\mathcal{H}_{0}+g \mu_{B} J_{z}\left(B_{0}+B_{m}\right)+\sum_{i} \frac{e^{2}}{8 m}\left(\boldsymbol{B} \times \boldsymbol{r}_{i}\right)^{2}+\lambda_{\mathrm{SO}} \boldsymbol{L} \cdot \boldsymbol{S}
$$

Desprezando-se o termo diamagnético e a interação spin-órbita, a equação (2.108) equivale ao hamiltoniano de um paramagneto em um campo magnético $B=B_{0}+B_{m}$. Isso permite que usemos o resultado de (2.93), substituindo $B$ por $B_{0}+B_{m}$ para obtermos

$$
\begin{array}{r}
M\left(B_{0}, T\right)=M_{S} \mathcal{B}_{J}(y) \\
y=\frac{g \mu_{B} J\left(B_{0}+\lambda M\left(B_{0}, T\right)\right)}{k T}
\end{array}
$$

Essas duas equações devem ser resolvidas simultaneamente, o que pode ser feito pelo método gráfico ou numericamente, procurando-se a intersecção entre as duas curvas para $M(y)$ dadas pelas equações (2.109) e (2.110). Em $B_{0}=0$, a equação (2.109) se reduz para

$$
M(y)=M_{S} \mathcal{B}_{J}(y)
$$

e a equação (2.110) se reduz a

$$
M(y)=\frac{k T}{g \mu_{B} J \lambda} y
$$

Um gráfico das duas curvas acima é apresentado na figura 2.2 para algumas temperaturas distintas. Observa-se que a partir de uma temperatura crítica, a temperatura de Curie, não há intersecção diferente da trivial e a magnetização é sempre nula. Abaixo da temperatura de Curie sempre existem duas soluções, uma positiva e uma negativa, e no limite $T \rightarrow 0$ a magnetização vale $M_{S}$.

Para encontrar a temperatura de ordenamento, igualam-se as derivadas das duas curvas em $y \rightarrow 0$. O limite para $y \rightarrow 0$ pode ser calculado através da expansão de $\mathcal{B}_{J}(y)$ 


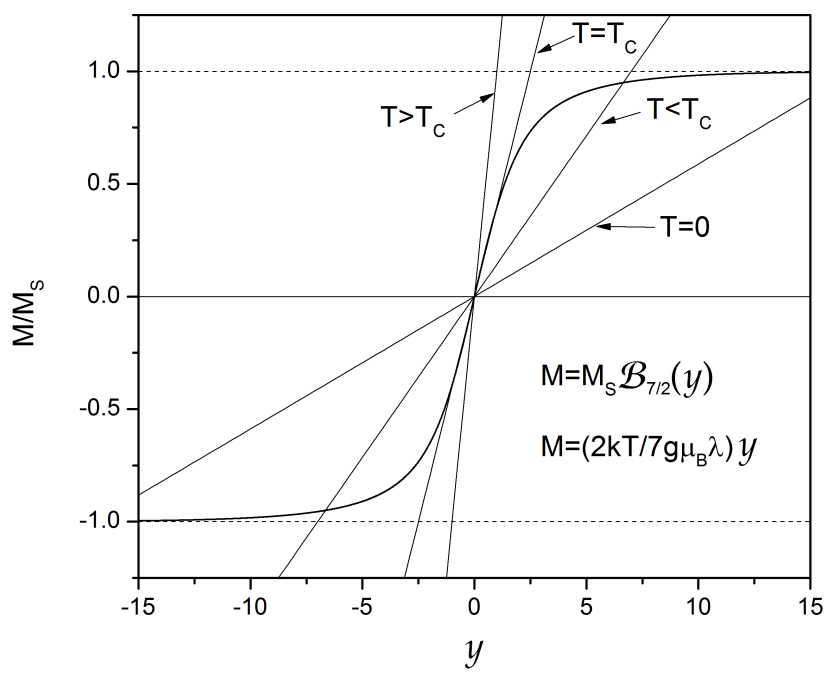

Figura 2.2: Solução gráfica das equações (2.109) e (2.110)

em uma série de potências em $y$, resultando em

$$
\lim _{y \rightarrow 0} \mathcal{B}_{J}^{\prime}(y)=\frac{J+1}{3 J}
$$

de forma que por um lado $M^{\prime}(y \rightarrow 0)=M_{S} \frac{J+1}{3 J} y$ e pelo outro lado $M^{\prime}(y)=\frac{k T}{g \mu_{B} J \lambda}$, portanto subtraindo-se as duas equações é possível determinar a temperatura de Curie como

$$
T_{C}=\frac{(J+1) g \mu_{B} \lambda M_{S}}{3 k}
$$

ou simplesmente

$$
T_{C}=C \lambda
$$

onde $C=\frac{(J+1) g \mu_{B} M_{S}}{3 k}$ é a mesma constante de Curie definida em (2.97).

Para valores finitos de $B_{0}$, sempre haverá uma magnetização não nula. Em temperaturas altas, quando $y \ll 1$, a função de Brillouin pode ser aproximada por $\mathcal{B}_{J}(y)=\frac{(J+1)}{3 J} y$, de forma que a magnetização fica dada por

$$
M\left(B_{0}, T\right)=M_{S} \frac{J+1}{3 J} \frac{g \mu_{B} J\left(B_{0}+\lambda M\left(B_{0}, T\right)\right)}{k T}=C \frac{B_{0}+\lambda M\left(B_{0}, T\right)}{k T}
$$

Isolando-se $M\left(B_{0}, T\right)$, essa equação pode ser rearranjada para

$$
M\left(B_{0}, T\right)=\frac{C}{T-T_{C}} B_{0}
$$


de maneira que a susceptibilidade vale

$$
\chi=\frac{C}{T-T_{C}}
$$

e tem a forma da lei de Curie-Weiss, a qual é válida na região $T>T_{C}$.

\section{Antiferromagnetismo}

Outros estados ferromagnéticos podem existir sem que haja nenhuma magnetização macroscópica observável. É o caso dos estados antiferromagnéticos AFM, descobertos por Louis Néel $[49,50]$, onde a interação de exchange acarreta num alinhamento antiparalelo dos spins da rede $(\lambda<0)$. O exemplo mais simples é o AFM de duas sub-redes, onde os elementos de cada sub-rede têm seus spins alinhados paralelamente, mas as magnetizações totais de cada grupo são alinhadas antiparalelamente, formando duas sub-redes FM interopostas, de modo que a magnetização total do sistema seja nula.

No AFM de duas sub-redes, cada sub-rede (nomeada A ou B) possui uma magnetização diferente e portanto são necessários dois campos moleculares para descrever o sistema no formalismo de Weiss: um campo efetivo devido a B que atua em elementos de A e um campo efetivo devido a A que atua em elementos de B. Esses campos efetivos podem ser parametrizados em função das magnetizações através da constante $\lambda$, definida como no estado FM, mas que nesse caso será negativa. Como átomos de A interagem apenas com átomos de B e vice-versa, pode-se definir os campos efetivos como

$$
\begin{aligned}
& \boldsymbol{B}_{A}=\boldsymbol{B}_{0}-|\lambda| \boldsymbol{M}_{B} \\
& \boldsymbol{B}_{B}=\boldsymbol{B}_{0}-|\lambda| \boldsymbol{M}_{A}
\end{aligned}
$$

Em $T=0$ e $B_{0}=0$, as duas sub-redes têm magnetizações de módulo $M_{S}$ e apontam em direções opostas, cancelando-se perfeitamente, de modo que a magnetização macroscópica total é nula

$$
M=M_{A}+M_{B}=0
$$


Quando leva-se em conta a aplicação de um campo magnético externo, o efeito do campo é o de tentar alinhar os spins para a sua direção. Se o campo magnético for aplicado perpendicularmente ao eixo antiferromagnético, o efeito do campo é o mesmo nas duas sub-redes, e as magnetizações são gradualmente alinhadas para a direção do campo, até que a partir de um certo valor crítico de campo $B_{S A T}$ todos os spins estão perfeitamente alinhados e o sistema encontra-se na fase FM induzida. Se o campo magnético é aplicado paralelamente ao eixo antiferromagnético, uma das sub-redes já encontra-se alinhada no sentido do campo e a outra encontra-se alinhada no sentido oposto ao do campo. Para campos pequenos, os spins permanecem alinhados, até que em certa intensidade crítica os spins saltam para uma nova configuração, numa transição conhecida como spin-flop. As magnetizações da duas sub-redes vão para direções opostas entre si e perpendiculares à direção do campo, como no primeiro caso, e a partir daí continuam a alinhar-se para a direção do campo até que a saturação seja atingida.

Na medida que aumenta-se a temperatura, flutuações térmicas agem no sistema, causando mudanças aleatórias nos estados dos spins. Em altas temperaturas essas flutuações térmicas sobrepujam o campo molecular que alinha os spins da rede e a magnetização das sub-redes se anula. Nessa situação, a aplicação de um campo externo induz uma pequena magnetização na direção do campo, a qual pode ser obtida como no caso do ferromagnetismo (equações (2.109) e (2.110)), como

$$
M=M_{S} \mathcal{B}_{J}\left(\frac{g \mu_{B} J\left(B_{0}-|\lambda| M\right)}{k T}\right)
$$

Como anteriormente, essa equação pode ser resolvida graficamente ou numericamente, encontrando a intersecção entre as curvas para a magnetização

$$
M=M_{S} \mathcal{B}_{J}(y) \text { e } M=\frac{k T}{g \mu_{B} J|\lambda|} y
$$

A temperatura a partir da qual as magnetizações das sub-redes anulam-se é denominada temperatura de Néel. Essa temperatura pode ser encontrada igualando-se as derivadas de ambas as curvas com $y \rightarrow 0$. O resultado é equivalente ao caso FM:

$$
T_{N}=\frac{(J+1) g \mu_{B}|\lambda| M_{S}}{3 k}
$$


Por outro lado, no limite de altas temperaturas, a magnetização de cada uma das sub-redes tende a zero, devido às flutuações térmicas dos spins. Entretanto, um campo magnético externo $B_{0}$ irá impor uma magnetização não nula paralela a si, logo de (2.121) temos para cada sub-rede

$$
M=\lim _{T \rightarrow \infty} M_{S} \mathcal{B}_{J}\left(\frac{g \mu_{B} J\left(B_{0}-|\lambda| M\right)}{k T}\right) \underbrace{=}_{(2.113)} M_{S} \frac{J+1}{3 J} \frac{g \mu_{B} J\left(B_{0}-|\lambda| M\right)}{k T}
$$

A equação (2.124) pode ser rearranjada e exprimida em termos da constante de Curie (2.97) como

$$
M=\frac{C}{T+C|\lambda|} B_{0}
$$

de forma que a susceptibilidade pode ser abarcada pela lei de Curie-Weiss, que tem como forma geral

$$
\chi \propto \frac{1}{T-\Theta}
$$

onde $\Theta$ é a temperatura de Weiss do sistema.

Nesse caso $\Theta=-T_{N}$ é negativa, ao passo que no ferromagnetismo $\Theta=T_{C}$ é positiva. A temperatura de ordenamento assim obtida vale $T_{N}=C|\lambda|$, ou

$$
T_{N}=\frac{N J(J+1) g^{2} \mu_{B}^{2}}{3 k}|\lambda|=\frac{M_{S}(J+1) g \mu_{B}|\lambda|}{3 k}
$$

que é a mesma temperatura obtida em (2.123).

A susceptibilidade para $T<T_{N}$ depende da orientação do campo externo em relação ao eixo fácil do material. Os casos notáveis são a susceptibilidade a campo externo paralelo ao eixo fácil $\chi_{\|}$e a susceptibilidade com o campo perpendicular ao eixo fácil $\chi_{\perp}$. No intervalo $0<T<T_{N}$, a susceptibilidade $\chi_{\perp}$ é constante, ao passo que $\chi_{\|}$ é zero em $T=0 \mathrm{~K}$ e cresce com a temperatura até atingir o valor de $\chi_{\perp}$ em $T_{N}$.

\section{Ferrimagnetismo}

Em alguns casos é possível que a magnetização das duas sub-redes não se cancelem por exato. Isso pode ocorrer, por exemplo, pela presença de mais de um tipo de espécie 
magnética, como é o caso das ferritas e das granadas de ferro. Uma magnetização total correspondente à diferença entre a magnetização de cada sub-rede caracteriza o estado como ferrimagnético (FiM). A teoria do ferrimagnetismo também foi desenvolvida por Louis Néel [51, 50], utilizando o formalismo do campo médio. Néel observou que diversas leis de magnetização podem ocorrer nesses estados, dependendo da proporção entre os íons magnéticos em cada sub-rede e da relação entre os coeficientes de campo médio, os quais estão relacionados com a magnitude da interação de exchange entre as espécies magnéticas. O formalismo de Néel considera que a magnetização total é a média ponderada das magnetizações de cada sub-rede:

$$
\boldsymbol{M}=p \boldsymbol{M}_{A}+q \boldsymbol{M}_{B}
$$

onde $p$ e $q=1-p$ são as frações de átomos magnéticos nas sub-redes $A$ e $B$ respectivamente. As magnetizações de cada sub-rede são descritas pelo sistema de equações

$$
\begin{aligned}
M_{A} & =M_{S} \mathcal{B}_{J}\left(\frac{M_{S}}{k T}\left(B_{0}-q \lambda_{a b} M_{B}+\lambda_{a a} M_{A}\right)\right) \\
M_{B} & =M_{S} \mathcal{B}_{J}\left(\frac{M_{S}}{k T}\left(B_{0}-p \lambda_{a b} M_{A}+\lambda_{b b} M_{B}\right)\right)
\end{aligned}
$$

onde $\lambda_{a b}$ é proporcional à interação entre as sub-redes A e B e $\lambda_{a a}$ e $\lambda_{b b}$ são proporcionais às interações intrasub-rede nas sub-redes $\mathrm{A}$ e $\mathrm{B}$ respectivamente. A solução dessas equações depende dos valores de $\lambda_{a a}, \lambda_{b b}$ e $\lambda_{a b}$ e pode originar uma grande variedade de curvas para as magnetizações das sub-redes.

Uma fato comum a todos os sistemas FiM é que em $T=0 \mathrm{~K}$ existe uma magnetização macroscópica, menor do que $M_{S}$. Além disso, na temperatura crítica $T_{C}$ a magnetização total se anula e o sistema passa por uma transição de fase FiM $\rightarrow$ PM. Entretanto, em alguns casos é possível que haja uma temperatura $T<T_{C}$ na qual a magnetização total se anula como consequência da equivalência das magnetizações das duas sub-redes opostas. Essa temperatura é chamada ponto de compensação e ocorre na própria fase FiM, não correspondendo a uma transição de fase. 


\subsubsection{Outros tipos de magnetismo}

Nas seções precedentes foram descritas as principais classes de magnetismo, contudo, uma grande variedade de arranjos magnéticos pode ocorrer além dos descritos, incluindo combinações desses estados. Alguns exemplos são o helimagnetismo, caracterizado por uma distribuição helicoidal dos momentos magnéticos, o speromagnetismo, caracterizado por uma distribuição aleatória e fixa das espécies magnéticas, o superparamagnetismo, onde nanopartículas FM ou FiM comportam-se analogamente a um paramagneto na presença de um campo magnético, e os vidros de spin, que apresentam uma dinâmica magnética própria, intermediária entre o FM e o PM. 


\subsection{Magnetismo nos Calcógenos de Európio}

\subsubsection{Calcógenos de európio}

Os calcogênios são os elementos químicos situados no grupo 16 da tabela periódica, a família do oxigênio. A nomenclatura provêm da palavra grega para o cobre, $\chi \alpha \lambda \kappa o ́ \varsigma$, também usada para designar as rochas ricas em cobre ou os óxidos de cobre. Esses elementos, oxigênio, enxofre, selênio, telúrio, polônio e o artificial livermório, podem ligar-se com elementos metálicos dando origem aos calcógenos metálicos, ou óxidos metálicos no caso do oxigênio. Doravante será utilizada a abreviação $X$ para designar qualquer um dos calcogênios. A característica comum a todos os calcogênios é que os mesmos possuem seis elétrons na camada de valência, de forma que a tendência desses materiais é a de receber dois elétrons, completando suas camadas de valência de acordo com a regra do octeto.

O európio, Eu, pertence aos elementos metálicos chamados de terras-raras, aqueles que compõem a série dos lantanídeos na tabela periódica, ou seja, os elementos de número atômico de 57 a 71 , e ainda os elementos escândio $(Z=21)$ e ítrio $(Z=39)$, considerados terras-raras por possuírem propriedades similares às dos elementos da série dos lantanídeos. Com número atômico 63, o Eu possui uma distribuição eletrônica [Xe $4 f^{7} 6 s^{2}$ e pode ligar-se ionicamente com um calcogênio, formando os compostos binários conhecidos como calcógenos de európio, EuX.

Os EuX são alvo de grande interesse em pesquisa básica e aplicada, sendo potenciais candidatos a aplicações tecnológicas envolvendo magnetismo, em virtude das propriedades magnéticas associadas aos íons de európio, $\mathrm{Eu}^{2+}$, os quais apresentam um momento magnético atômico bastante elevado $\left(\mu \simeq 7 \mu_{B}\right)$. Os cristais iônicos de EuX são intrinsecamente magnéticos e possuem uma alta densidade de íons $\mathrm{Eu}^{2+}$ na rede, conferindo a esses materiais um dos maiores momentos magnéticos encontrados em um semicondutor.

As diversas propriedades dos EuX estão relacionadas com sua estrutura molecular e níveis de energia eletrônicos. Entender essa relação é um objetivo que vem atraindo a 
atenção de pesquisadores desde os anos 60, quando foram estudadas a ordem magnética [7, 52], o calor específico [53] e a absorção de luz [54] EuX. Os trabalhos dessa época foram realizados com amostras amorfas ou cristalinas de baixa pureza. Em meados da década de 70, o espectro de absorção óptica foi obtido em monocristais de EuX $[55,21,56]$, apresentando uma janela de transparência na região visível do espectro eletromagnético. O modelo teórico até então utilizado para a absorção consiste da transição de um elétron de um nível de valência $4 f$ bem localizado para uma banda de condução $5 d$ construída a partir de níveis atômicos do Eu, separados por um campo cristalino de simetria octaédrica [9]. Os experimentos da época não mostraram total concordância com esse modelo, pois no mesmo não fora incluída a formação de domínios magnéticos, que só foi levada em conta numa recente re-examinação do modelo, que mostrou boa concordância com resultados experimentais [26].

A seguir, a estrutura eletrônica dos EuX será apresentada e a partir disso será demonstrado como essa estrutura origina o magnetismo nesses materiais. Nos EuX, a ligação química é do tipo iônica e dá-se quando o par de elétrons de valência $6 s^{2}$ do átomo de európio migra para a órbita do calcogênio, de forma que esses átomos ficam ionizados e mantêm-se unidos pelo potencial atrativo entre as cargas positivas do $\mathrm{Eu}^{2+}$ e negativas do $\mathrm{X}^{2-}$. A agregação desses dois materiais num composto binário iônico pode dar origem a um sólido cristalino, de estrutura cúbica de face centrada $(f c c)$, idêntica à estrutura do $\mathrm{NaCl}$, como ilustrado na figura 2.3.

A distribuição eletrônica do cátion $\mathrm{Eu}^{2+}$ é $[\mathrm{Xe}] 4 f^{7}$, onde [Xe] representa a distribuição em camadas eletrônicas completas, equivalentes à distribuição eletrônica de um átomo de xenônio neutro. Os sete elétrons restantes distribuem-se no orbital $f$, que pode ser aproximado como o nível fundamental de um átomo com um núcleo efetivo, que inclui o núcleo atômico e as camadas eletrônicas completas. No estado fundamental, os sete elétrons da camada $f$ distribuem-se de acordo com as regras de Hund. A primeira regra diz que os spins individuais, $s_{i}$, são distribuídos de forma a maximizar a multiplicidade de spin, $2 S+1$, onde

$$
S=\sum_{i=1}^{7} m_{s i}
$$




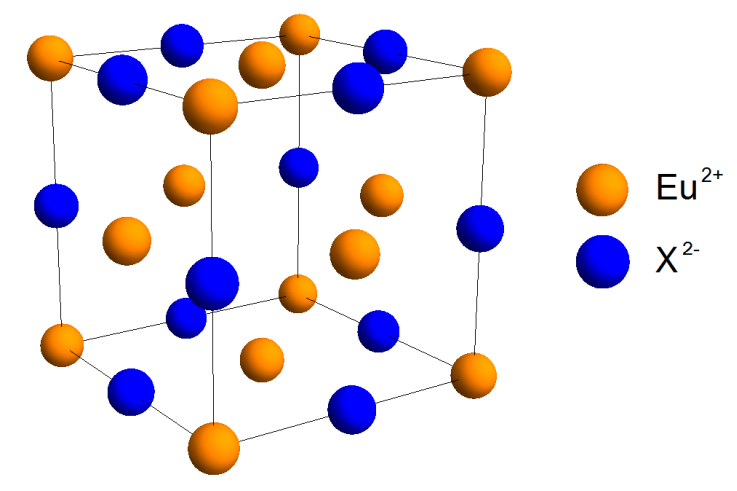

Figura 2.3: A cúbica de face centrada, também conhecida como estrutura rocksalt, por ser a estrutura química do sal de cozinha, o mais comum dos cristais iônicos.

é o spin total do átomo. A maior multiplicidade é obtida quando os sete spins possuem a mesma projeção, $m_{S}=+\frac{1}{2}$ totalizando $S=\frac{7}{2}$. O momento angular orbital, $L$, de um subnível de número quântico $\ell$ é dado por

$$
L=\sum_{-\ell}^{\ell} m_{\ell}
$$

onde $m_{\ell}$ é a projeção no eixo $z$ do momento angular orbital de cada elétron. Como o subnível $f$ comporta 14 elétrons, o mesmo encontra-se semi-preenchido. Pela primeira regra de Hund, todos os sete elétrons tem a mesma projeção de spin e portanto cada elétron tem uma projeção $m_{\ell}$ diferente, de maneira que a soma em (2.132) se anula. O momento angular total, $\boldsymbol{J}=\boldsymbol{L}+\boldsymbol{S}$, é dado simplesmente pelo termo de spin, sendo a primeira regra de Hund suficiente para determinar o estado fundamental do $\mathrm{Eu}^{2+}, \mathrm{o}$ qual na notação espectroscópica ${ }^{2 S+1}(S, P, D, F)_{J}$ é descrito por um termo ${ }^{8} S_{\frac{7}{2}}$.

O fato de todos os spins da camada possuírem a mesma projeção $m_{s}$, implica numa elevada magnitude do momento magnético atômico do íon $\mathrm{Eu}^{2+}$, o qual classicamente é dado por $\mu=g \mu_{B} S=7 \mu_{B}$ e do ponto de vista quântico por $\mu=g \mu_{B} \sqrt{S(S+1)} \sim 8 \mu_{B}$. $\mathrm{O}$ ânion $\mathrm{X}^{2-}$ por outro lado, tem sua camada de valência completa com oito elétrons, de modo que os íons dos calcogênios não contribuem para o magnetismo dos EuX. Os íons $\mathrm{Eu}^{2+}$ num cristal $\mathrm{EuX}$ interagem por exchange e quando a energia de exchange é maior do que a energia das flutuações térmicas, essa interação origina ordenamentos 
magnéticos dos spins dos íons $\mathrm{Eu}^{2+}$. Esses ordenamentos serão analisados através do formalismo do campo médio, o qual foi descrito na seção 2.2.

\subsubsection{Modelo do campo médio para o magnetismo nos EuX}

Em geral a interação de exchange tem alcance limitado e por isso não deve ser expressiva para um par de sítios muito distantes. Neste trabalho serão consideradas interações com os primeiros e segundos vizinhos, uma aproximação que se mostrará suficiente para explicar algumas das propriedades magnéticas dos EuX. Um átomo qualquer em uma rede $f c c$ de lado $l$, possui 12 primeiros vizinhos localizados a uma distância $l \sqrt{2}$ ao longo das diagonais da cúbica e 6 segundos vizinhos localizados a uma distância $l$ ao longo dos eixos da cúbica (ver figura 2.4). Para que a aproximação do campo médio

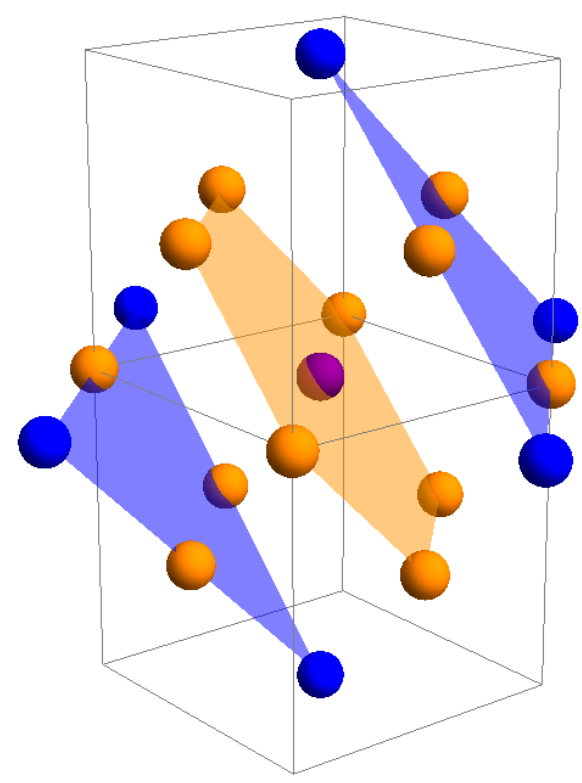

Figura 2.4: Um átomo na cúbica-de-face-centrada, representado na cor roxa, possui 12 primeiros vizinhos, representados em laranja e 6 segundos vizinhos, em azul.

possa ser implementada, é necessário dividir a rede em um número de sub-redes que garanta que não haja interações entre elementos da mesma sub-rede. Na seção 2.2.2 foi explorado o exemplo de uma rede dividida em duas sub-redes, A e B, onde elementos de A só interagem com elementos de B e vice-versa. Na rede cúbica de face centrada, 
essa condição não pode ser verificada com apenas duas sub-redes para interações de primeiros e segundos vizinhos. A condição de não interação intrasub-rede pode ser contemplada dividindo-se a rede em oito sub-redes interpenetrantes, de forma que cada íon $\mathrm{Eu}^{2+}$ está contido em uma das sub-redes do material e possui dois primeiros vizinhos em seis das demais sub-redes e os seis segundos vizinhos na sub-rede restante.

O campo efetivo que atua sobre um átomo da $i$-ésima sub-rede é a composição do campo molecular devido às interações de exchange com os sítios vizinhos. Seguindo o formalismo apresentado na seção 2.2.2, o campo molecular devido a cada sub-rede é proporcional à magnetização da mesma, de forma que

$$
\boldsymbol{B}_{i}=\boldsymbol{B}_{0}+\sum_{j=1}^{8} \lambda_{i j} \boldsymbol{M}_{j}
$$

onde $M_{j}$ é a magnetização da j-ésima sub-rede e as constantes do campo molecular são obtidas por generalização da relação (2.107) como (cf. referência [57])

$$
\lambda_{i j}=\frac{8\left(2 z_{i j} J_{i j}\right)}{N g^{2} \mu_{B}^{2}}
$$

onde $z_{i j}$ é o número de spins equivalentes (primeiros vizinhos, segundos vizinhos, etc.) que circundam o sítio $i$ e cuja interação de exchange com o sítio $i$ é descrita pela mesma constante de exchange $J_{i j}$. O fator 8 é o número de sub-redes na rede $f_{c c}$, conforme discutido acima, que garante que não haja interações intrasub-rede, isto é, $\lambda_{i i}=0$. Por simetria, $J_{i j}=J_{j i}$ e portanto $\lambda_{i j}=\lambda_{j i}$.

A magnetização de cada sub-rede depende do campo externo e do campo molecular devido às demais sub-redes. Num sistema onde as magnetizações de todas as sub-redes apontam na direção do campo, o resultado (2.109) pode ser aproveitado, de forma que a magnetização da sub-rede $i$, para um campo magnético orientado na direção $z$, é dada por

$$
M_{i}=\frac{M_{S}}{8} \mathcal{B}_{J}\left(\frac{g \mu_{B} J}{k T} B_{i}\right)
$$

onde $M_{S}$ foi substituído por $M_{S} / 8$ por tratar-se de uma sub-rede que contém um oitavo dos átomos magnéticos do cristal. Como $B_{i}$ depende das magnetizações das outras 
sub-redes, a equação (2.135) representa um sistema de oito equações acopladas, cuja solução é bastante complicada.

No limite de altas temperaturas, mesmo num sistema com anisotropia magnética, as magnetizações de todas as sub-redes apontam na direção do campo e podemos utilizar a expressão (2.135) para as magnetizações de cada sub-rede:

$$
M_{i}=\frac{M_{S}}{8} \mathcal{B}_{J}\left(\frac{g \mu_{B} J}{k T} B_{i}\right)
$$

Nesse regime de temperaturas é possível utilizarmos o primeiro termo da expansão em série de potências da função de Brillouin $\mathcal{B}_{J}(y)=\frac{J(J+1)}{3 J} y$, simplificando o sistema (2.135) para

$$
M_{i}=\frac{C}{8 T} B_{i}
$$

onde $B_{i}=B_{0}+\sum_{j=1}^{8} \lambda_{i j} M_{j}$ e $C=\frac{g \mu_{B}(J+1) M_{S}}{3 k}$ é a constante de Curie (2.97).

Somando-se as oito equações (2.137) obtemos

$$
\sum_{i=1}^{8} M_{i}=\frac{C}{8 T} \sum_{i} B_{i}=\frac{C}{8 T} \sum_{i}\left(B_{0}+\sum_{j} \lambda_{i j} M_{i}\right)
$$

Como $\lambda_{i j}=\lambda_{j i}$ pode-se trocar a ordem do somatório da equação (2.138), que pode ser reescrita como

$$
\sum_{i=1}^{8} M_{i}=\frac{C}{8 T} \sum_{j} B_{0}+\frac{C}{8 T} \sum_{i} \sum_{j} \lambda_{i j} M_{i}=\sum_{i} M_{i} \frac{C}{8 T} \sum_{j} \lambda_{i j}+\frac{C}{T} B_{0}
$$

ou

$$
\sum_{i=1}^{8} M_{i}\left(1-\frac{C}{8 T} \sum_{j=1}^{8} \lambda_{i j}\right)=\frac{C}{T} B_{0}
$$

A expressão entre parênteses acima independe de $i$, devido à equivalência translacional dos átomos da rede. Isto é, cada átomo tem o mesmo número e tipo de interação que qualquer outro, logo o termo entre parêntese pode ser extraído do somatório:

$$
\left(1-\frac{C}{8 T} \sum_{j=1}^{8} \lambda_{i j}\right) \sum_{i=1}^{8} M_{i}=\frac{C}{T} B_{0}
$$


Sabendo-se que a magnetização total é dada por

$$
M=\sum_{i=1}^{8} M_{i}
$$

a equação (2.141) pode ser escrita como

$$
M=\frac{C B_{0}}{\left(1-\frac{C}{8 T} \sum_{j=1}^{8} \lambda_{i j}\right) T}
$$

que fornece exatamente a lei de Curie-Weiss

$$
\chi=\frac{C}{T-\Theta}
$$

com

$$
\Theta=\frac{C}{8} \sum_{j=1}^{8} \lambda_{i j}
$$

A constante $\Theta$, chamada temperatura de Weiss [45], pode ser ser obtida para um rede $f c c$ substituindo-se em (2.145) a constante $\lambda_{i j}$ definida em (2.134) e a constante de Curie definida em (2.97), resultando em

$$
\Theta=\frac{2 J(J+1)}{3 k} \sum_{j=1}^{8} z_{i j} J_{i j}
$$

Pela divisão em oito sub-redes, um átomo $i$ de Eu possui dois de seus primeiros vizinhos contidos em seis das sub-redes e todos os seis segundos vizinhos contidos na sub-rede restante, de forma que a soma em (2.146) resulta em $\sum_{j=1}^{8} z_{i j} J_{i j}=6 \times 2 J_{1}+6 J_{2}$, onde $J_{1}$ e $J_{2}$ são as constantes de exchange de um átomo com seus primeiros e segundos vizinhos, respectivamente. Dessa maneira a temperatura de Weiss para os EuXé obtida como

$$
\Theta=\frac{2 J(J+1)}{3 k}\left(12 J_{1}+6 J_{2}\right)
$$

Ainda que a fórmula (2.143) descreva o comportamento da magnetização em função da temperatura no regime de altas temperaturas, o parâmetro $\Theta$ não caracteriza a transição entre desordem magnética e ordem magnética. A transição entre ordem e desordem magnéticas é caracterizada pela temperatura $T_{N}$, acima da qual a magnetização 
de cada uma das sub-redes é zero e abaixo da qual é não nula. Para determinar essa temperatura $T_{N}$, voltamos à equação (2.137) e escrevemos explicitamente o sistema de equações ali contido $(i=1,2, \ldots, 8)$.

$$
\begin{aligned}
M_{1}-\frac{C}{8 T} \lambda_{12} M_{2}-\cdots-\frac{C}{8 T} \lambda_{18} M_{8} & =\frac{C}{8 T} B_{0} \\
& \vdots \\
-\frac{C}{8 T} \lambda_{81} M_{1}-\frac{C}{8 T} \lambda_{82} M_{2}-\cdots+M_{8} & =\frac{C}{8 T} B_{0}
\end{aligned}
$$

O sistema de equações acima só tem soluções não nulas para $M_{i}$ se e somente se o seu determinante característico for nulo, o que define uma equação para as temperaturas críticas de Néel.

Seja a sub-rede que contém os seis segundos vizinhos de um átomo na sub-rede $i=1$ aquela rotulada pelo índice $j=5$. Nessa descrição, de (2.134) obtemos

$$
\lambda_{15}=\frac{8 \times 2 \times 6 J_{2}}{N g^{2} \mu_{B}^{2}} \text { e } \lambda_{1 j \neq 5}=\frac{8 \times 2 \times 2 J_{1}}{N g^{2} \mu_{B}^{2}}
$$

Definindo-se

$$
\lambda_{1}=\frac{24 J_{1}}{N g^{2} \mu_{B}^{2}} \text { e } \quad \lambda_{2}=\frac{12 J_{2}}{N g^{2} \mu_{B}^{2}}
$$

obtemos $\lambda_{15}=\frac{4}{3} \lambda_{1}$ e $\lambda_{1 j \neq 5}=8 \lambda_{2}$. Para as demais sub-redes pode-se partir dessa escolha e definir as demais constantes de interação obedecendo um critério de ciclicidade. Por exemplo, para um átomo na sub-rede $i=2$, os seis segundos vizinhos estão contidos na sub-rede $j=6$, de forma que

$$
\lambda_{26}=\frac{8 \times 2 \times 6 J_{2}}{N g^{2} \mu_{B}^{2}}=\lambda_{15}=8 \lambda_{2} \text { e } \lambda_{2 j \neq 6}=\frac{8 \times 2 \times 2 J_{1}}{N g^{2} \mu_{B}^{2}}=\lambda_{26}=\frac{4}{3} \lambda_{1}
$$

e assim por diante para as demais sub-redes.

Multiplicando todos os elementos de (2.148) por $\frac{8 T}{C}$ e definindo-se

$$
\begin{aligned}
& a_{0} \equiv \frac{8 T}{C} \\
& a_{1} \equiv-\frac{4}{3} \lambda_{1} \\
& a_{2} \equiv-8 \lambda_{2}
\end{aligned}
$$


a matriz dos coeficientes do sistema (2.148) fica

$$
Q=\left(\begin{array}{llllllll}
a_{0} & a_{1} & a_{1} & a_{1} & a_{2} & a_{1} & a_{1} & a_{1} \\
a_{1} & a_{0} & a_{1} & a_{1} & a_{1} & a_{2} & a_{1} & a_{1} \\
a_{1} & a_{1} & a_{0} & a_{1} & a_{1} & a_{1} & a_{2} & a_{1} \\
a_{1} & a_{1} & a_{1} & a_{0} & a_{1} & a_{1} & a_{1} & a_{2} \\
a_{1} & a_{1} & a_{1} & a_{1} & a_{0} & a_{1} & a_{1} & a_{1} \\
a_{2} & a_{1} & a_{1} & a_{1} & a_{1} & a_{0} & a_{1} & a_{1} \\
a_{1} & a_{2} & a_{1} & a_{1} & a_{1} & a_{1} & a_{0} & a_{1} \\
a_{1} & a_{1} & a_{2} & a_{1} & a_{1} & a_{1} & a_{1} & a_{0}
\end{array}\right)
$$

Fazendo $\operatorname{det} Q=0$ obtemos três raízes reais para o sistema, determinadas por

$$
\begin{array}{lcl}
\text { (a) } a_{0}=-6 a_{1}-a_{2} \rightarrow & T=C\left(\lambda_{1}+\lambda_{2}\right) \\
\text { (b) } a_{0}=2 a_{1}-a_{2} \rightarrow & T=C\left(-\frac{\lambda_{1}}{3}+\lambda_{2}\right) \\
\text { (c) } \quad a_{0}=a_{2} \rightarrow & T=-C \lambda_{2}
\end{array}
$$

Cada uma dessas raízes corresponde a um sistema diferente que é possível e indeterminado, ou seja, admite infinitas soluções. Entretanto é possível obter relações entre as magnetizações de cada sub-rede. Para a raíz (a), as soluções implicam em

$$
M_{1}=M_{2}=M_{3}=M_{4}=M_{5}=M_{6}=M_{7}=M_{8}
$$

o que corresponde a um sistema ordenado ferromagneticamente, isto é, a magnetização de todas as sub-redes apontam na mesma direção e tem-se uma temperatura de Curie

$$
T_{C}=\frac{63\left(2 J_{1}+J_{2}\right)}{k}=\Theta
$$

Para a raiz (b), as soluç̃oes tem forma

$$
\begin{aligned}
& M_{1}=M_{5} \\
& M_{2}=M_{6} \\
& M_{3}=M_{7} \\
& M_{4}=M_{8}=-M_{1}-M_{2}-M_{3}
\end{aligned}
$$


Essas condições podem gerar mais de um ordenamento magnético, entretanto se for feita a escolha $M_{1}=M_{2}=-M_{3}=-M_{4}$, o ordenamento obtido é o chamado antiferromagnético do tipo I, onde os spins contidos nos planos definidos pelos eixos da cúbica (por exemplo os planos $x-y$ ) apresentam ordenamento ferromagnético entre si, entretanto as magnetizações dos planos vizinhos apontam em direções opostas, de forma que o material como um todo é antiferromagnético. A temperatura de Néel correspondente a esse caso vale

$$
T_{N}^{A F M I}=\frac{-42 J_{1}+63 J_{2}}{k}
$$

Para o caso $(c)$ escolhendo-se a restrição

$$
\begin{aligned}
& M_{1}=-M_{5} \\
& M_{2}=-M_{6} \\
& M_{3}=-M_{7} \\
& M_{4}=-M_{8}
\end{aligned}
$$

obtém-se o arranjo antiferromagnetico de tipo II, onde assim como no tipo I, formam-se planos ferromagnéticos distribuídos alternadamente com magnetizações inversas. A diferença é que nesse caso a família de planos FM é formada pelos planos perpendiculares às diagonais da cúbica. Com essa restrição, a temperatura de ordenamento é definida por

$$
T_{N}^{A F M I I}=-\frac{63 J_{2}}{k}
$$

Nesse caso o exchange de primeiros vizinhos não influencia a temperatura de Néel.

Através do simples modelo de Weiss do campo efetivo, foi possível chegar a diferentes ordenamentos magnéticos que podem ser assumidos pelos EuX abaixo das temperaturas críticas determinadas em função das constantes de exchange $J_{1}$ e $J_{2}$.

Na figura 2.5 a) é possível observar os planos ferromagnéticos e os átomos vizinhos de primeira e segunda ordem de um dado sítio da rede. O estado magnético é então determinado pela distribuição relativa da magnetização total desses planos. Além 


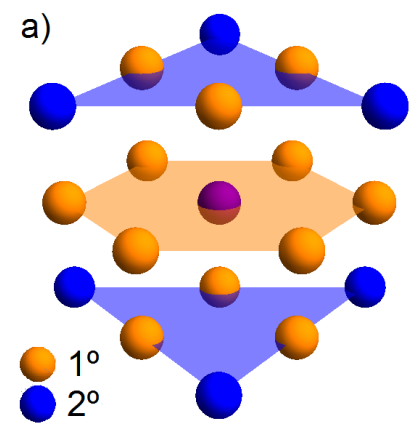

b)
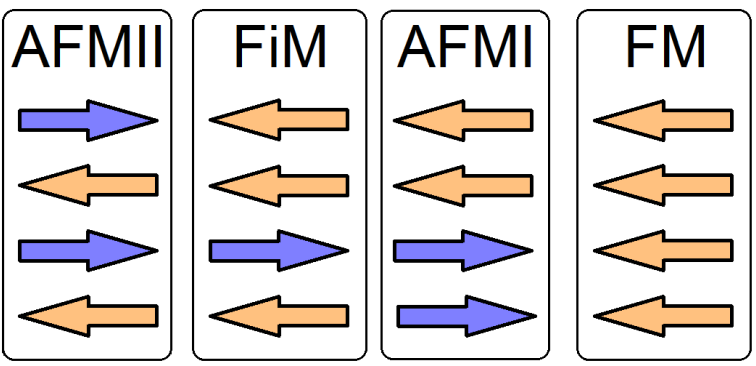

Figura 2.5: Os estados magnéticos dos EuX

dos ordenamentos derivados das escolhas feitas para resolver o sistema de equações (FM e AFMII), outras ordens magnéticas estão presentes nos EuX, sempre formadas por pelos sucessivos planos FM, que podem distribuir-se em padrões $\uparrow \uparrow \downarrow \downarrow$ formando antiferromagnetismo de tipo I (AFMI) [58], ou $\uparrow \uparrow \downarrow$ caracterizando um ordenameno ferrimagnetico (FiM). Os diversos possíveis ordenamentos magnéticos dos EuX estão ilustrados na figura $2.5 \mathrm{~b}$ ).

Conhecendo os ordenamentos magnéticos que ocorrem em cada $\mathrm{EuX}$, as respectivas temperaturas críticas e a evolução da orientação dos spins em função do campo magnético aplicado, pode-se determinar o valor das constantes de exchange que se adequam ao modelo do campo médio. A seguir as constantes de exchange e os ordenamentos magnéticos serão analisados para cada um dos EuX.

\subsubsection{EuO e EuS}

Em trabalho de 1961, Matthias, Bozorth e Van Vleck [59] demonstraram através de medidas da susceptibilidade magnética que o EuO apresenta ordenamento FM dos spins da rede, com uma temperatura de Curie de $77 \mathrm{~K}$. O mesmo tipo de ordenamento foi encontrado para o $\operatorname{EuS}[6,60]$, cuja temperatura de Curie determinada foi de $18 \mathrm{~K}$. Contudo, ainda em estágios iniciais, esses trabalhos não chegaram a uma conclusão acerca das constantes de exchange $J_{1}$ e $J_{2}$. Posteriormente, Dietrich et al. [61] encontraram por calorimetria os valores $J_{1} / k=0.61 \mathrm{~K}$ e $J_{2} / k=0.12 \mathrm{~K}$ para o EuO e $J_{1} / k=0.23 \mathrm{~K}$ e $J_{2} / k=-0.10 \mathrm{~K}$ para o EuS. A teoria do campo médio pode ser empregada nesse sistema 
considerando-se a solução 2.154, a qual prevê uma temperatura crítica $T_{C}=\frac{63\left(2 J_{1}+J_{2}\right)}{k}$. Com os valores experimentais para $J_{1}$ e $J_{2}$ as temperaturas de Curie ficam $T_{C}=84 \mathrm{~K}$ para o EuO e $T_{C}=22 \mathrm{~K}$ para o EuS. Essas temperaturas estão ligeiramente acima (10\% e 20\%) das encontrados experimentalmente, entretanto concordam qualitativamente.

\subsubsection{EuSe}

O seleneto de európio é o EuX que apresenta a mais complexa estrutura magnética, manifestando ao menos cinco possíveis ordenamentos magnéticos. O estado magnético de equilíbrio desse material é muito sensível a mudanças nas variáveis pressão, temperatura e campo magnético externo. Isso pode ser compreendido pelo fato de que a interação antiferromagnética de segundos vizinhos é da mesma ordem da interação ferromagnética de primeiros vizinhos $\left|\frac{J_{1}}{J_{2}}\right| \simeq 1$, e por haver o mesmo número (6) de primeiros e de segundos vizinhos nos planos adjacentes, há um equilíbrio entre as duas interações que pode ser facilmente perturbado por mudanças nas variáveis intensivas do sistema. Em pressão atmosférica e campo nulo, Griessen et. al mostraram em trabalho de 1971 [58] que monocristais de EuSe apresentam três diferentes tipos de ordem magnética no intervalo de temperaturas entre $T=0 \mathrm{~K}$ e a temperatura de Néel $T=4.6$ K: o AFMII, o AFMI e o FiM. Callen e de Moura em 1977 [62] e mais recentemente em amostras epitaxiais Díaz et al. [63] encontraram as mesmas fases magnéticas e também a coexistência de mais de uma delas simultaneamente. Nesses trabalhos foi revelada também uma sensibilidade do sistema ao histórico da temperatura, isso é, experimentos feitos com temperatura crescente ou decrescente, levam a diferentes temperaturas de transição. Essas temperaturas de transição variam um pouco na literatura, entretanto existem intervalos de consenso, notadamente observa-se que $T=4.5 \mathrm{~K}$ está abaixo de $T_{N}$ e que abaixo de $T=1.8 \mathrm{~K}$ o ordenamento é AFMII. Esses intervalos serão importantes na escolha das temperaturas utilizadas nos experimentos.

O modelo do campo médio não é capaz de prever fases magnéticas intermediárias entre a fase de maior temperatura de ordenamento e a fase em $T=0 \mathrm{~K}$, a menos que as constantes de exchange possam variar com a temperatura [57]. No EuSe a 
presença de três fases magnéticas para $T<T_{N}$ pode ser analisada no modelo do campo médio desde que assuma-se que as constantes de exchange possam depender da temperatura. De fato essa dependência existe, pois a distância interplanar sofre variações quando varia-se a temperatura. Em $T=0 \mathrm{~K}$ o arranjo é AFMII e os spins estão contidos nos planos epitaxiais perfeitamente alinhados. Em temperaturas finitas as flutuações térmicas tendem a desfazer o ordenamento magnético numa razão proporcional à temperatura. Em $T_{N}^{1}$ uma contração da rede na direção [111] é observada, juntamente com um rearranjo magnético do sistema, que passa para a fase FiM. A temperatura $T_{N}^{1}$ varia na literatura, de $T_{N}^{1}=1.8 \mathrm{~K} \mathrm{em} \mathrm{[58]} \mathrm{a} T_{N}^{1}=3.2 \mathrm{~K}$ em [63]. Adicional aumento da temperatura leva a uma nova contração da rede em $T=T_{C}$, quando o ordenamento passa a ser AFMI. A partir dessa temperatura não existem outros ordenamentos magnéticos estáveis. Quando a temperatura de Néel é atingida, uma expansão na rede é observada, caracterizando a transição da fase AFMI para a fase paramagnética, a partir de onde a distância interplanar cresce linearmente com a temperatura [63].

A aplicação de um campo magnético externo também altera a ordem dos spins através da interação Zeeman, que tende a alinhar os spins à direção do campo. A anisotropia magnética e o acoplamento entre os spins podem oferecer resistência ao efeito do campo, entretanto a partir de uma magnitude crítica $B_{C}$ a energia da interação Zeeman predomina e todos os spins da rede são alinhados à direção do campo. Os estados intermediários para $0<B<B_{C}$ dependem da ordem magnética em cada intervalo de temperatura. Abaixo de $T_{N}$, o aumento do campo leva o sistema da ordem AFM(I ou II) para a ordem FiM e da ordem FiM para a FM. As curvas da magnetização e da espessura da amostra em função do campo magnético para monocristais de EuSe reportados em [58], mostraram degraus que ocorrem nos mesmos valores de campo tanto na magnetização quanto na espessura, para duas temperaturas diferentes, o que indica que as transições magnéticas induzidas por campo, assim como as induzidas por variação na temperatura, são acompanhadas por deformações na rede. Com aumento do campo magnético a partir da fase AFMI, uma expansão na rede esta- 
biliza a fase FiM, ao contrário do que acontece na transição $\mathrm{AFMII} \rightarrow \mathrm{FiM}$. Essa expansão está de acordo com a contração observada na transição FiM $\rightarrow$ AFMI induzida pelo aumento da temperatura. Em qualquer dos casos, o aumento do campo a partir da fase FiM, leva o sistema gradualmente da configuração $\uparrow \uparrow \downarrow$ para a condição de saturação

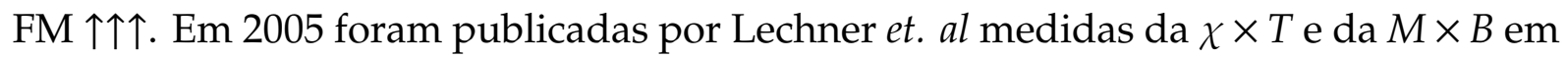
amostras epitaxiais de EuSe, obtidas com um equipamento do tipo SQUID. A partir dos picos na susceptibilidade em valores fixos de campo, foi montado um diagrama B-T de fases do EuSe, o qual mostrou-se muito semelhante ao mesmo diagrama obtido em [58] com tipos diferentes de amostras e através de métodos experimentais distintos. Esses resultados podem ser observados no diagrama B-T da figura 2.6. A origem do intrincado diagrama de fases no EuSe foi atribuída a efeitos além do simples modelo de Heisenberg na referência [64], onde foram propostos outros mecanismos de interação como exchanges de terceiros vizinhos, biquadrático, dipolar e pseudo-dipolar.

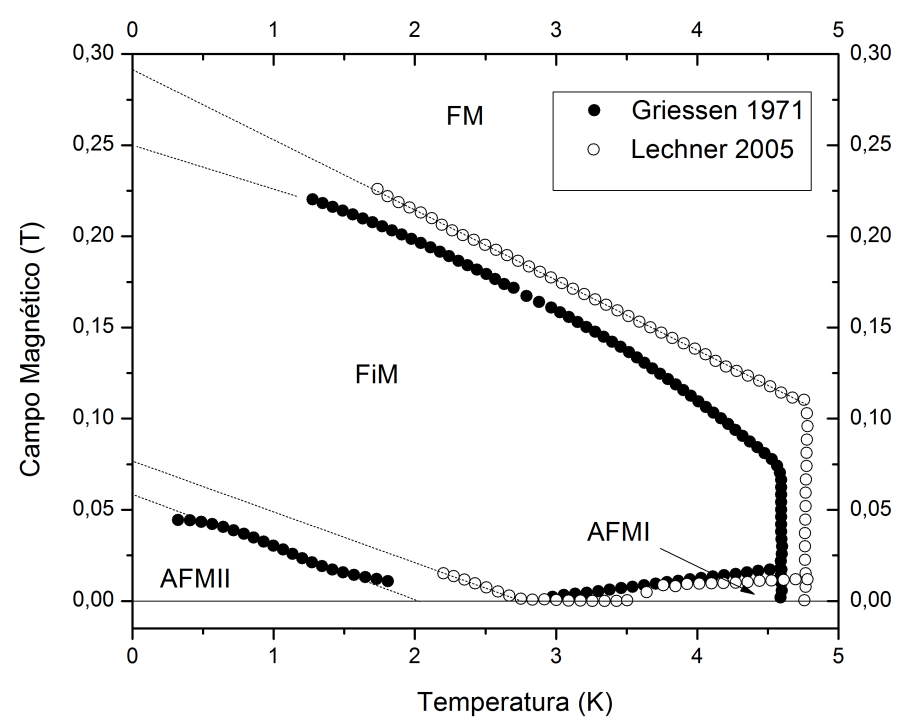

Figura 2.6: Diagrama de fases magnéticas do EuSe obtido para o campo na geometria Voigt por Griessen et. al em 1971 em monocristais e Lechner et. al. em 2005 em amostas epitaxiais. As linhas finas são extrapolações.

A aproximação do campo médio pode ser utilizada para modelar as temperaturas 
de ordenamento, resultando em valores experimentais para as constantes de exchange. Para a fase FiM, um dado sítio da rede possui 9 primeiros vizinhos orientados paralelamente e 3 antiparalelamente. Os segundos vizinhos estão divididos três a três nos dois planos adjacentes, e orientados em direções opostas, de forma que as forças exercidas pelos mesmos no sítio da rede se cancelam, portanto

$$
T_{C}^{F i M}=\frac{2 S(S+1)}{3 k} 9 J_{1}+3 J_{2}-3 J_{2}-3 J_{1}=\frac{63 J_{1}}{k}
$$

Utilizando o valor $T_{C}=2.8 \mathrm{~K}$ [58], obtém-se $J_{1} / k=0.044 \mathrm{~K}$.

Na fase AFMI esses planos distribuem-se segundo um ordenamento $\uparrow \uparrow \downarrow \downarrow$, o que corresponde a uma situação igual ao caso FiM considerando-se apenas primeiros e segundos vizinhos

$$
T_{N}^{A F M I}=\frac{63 J_{1}}{k}
$$

Sabendo-se que $T_{N}=4.6 \mathrm{~K}$ a integral de exchange resulta em $J_{1} / k=0.073 \mathrm{~K}$.

Para o ordenamento AFMII, as interações com primeiros vizinhos é que se cancelam, de modo que

$$
T_{N}^{A F M I I}=-\frac{63 J_{2}}{k}
$$

Utilizando o valor $T_{N}^{1}=1.8 \mathrm{~K}$, a integral de exchange de segundos vizinhos resulta em $J_{2} / k=-0.029 \mathrm{~K}$.

\subsubsection{EuTe}

O EuTe é o calcógeno de európio de maior distância entre os íons Eu ${ }^{2+}$. Nesse material a integral de exchange de segundos vizinhos, $J_{2}$ é predominante, pois a mesma decresce em razão menor do que $J_{1}$ quando aumenta-se a distância interatômica. O sinal negativo de $J_{2}$ favorece um alinhamento antiferromagnético dos spins da rede, em temperaturas abaixo da temperatura de Néel. Através de espectros de difração de nêutrons, Will et al. [65] reportaram um ordenamento AFMII, onde os spins da rede ordenam-se em planos FM, perpendiculares às diagonais da FCC. Battles e Everett reportaram em trabalho 
de 1970 [66], que os spins do EuTe ficam contidos nos planos FM em virtude de uma anisotropia magnética, a qual também resulta em direções preferenciais para os spins dentro desses planos. Os sucessivos planos FM distribuem-se ao longo da direção [111] (ou das demais diagonais da cúbica) num padrão $\uparrow \downarrow \uparrow \downarrow$ dos spins totais de cada plano, de forma que o sistema pode ser dividido em duas sub-redes interpenetrantes, idênticas, exceto pelo sentido da magnetização que é oposto. Esse ordenamento magnético no modelo do campo médio corresponde à solução (2.156), a qual, para $J=7 / 2, g=2 \mathrm{e}$ com as definições (2.97) e (2.150), prevê uma temperatura de ordenamento

$$
T_{N}^{A F M I I}=-\frac{63 J_{2}}{k}
$$

Em trabalho de 1972 [67], a temperatura de Néel do EuTe foi obtida experimentalmente por medidas da susceptibilidade magnética, resultando em $T_{C}=9.6(1) \mathrm{K}$, o que leva a uma constante de exchange $J_{2} / k=-0.15 \mathrm{~K}$.

A aplicação de um campo magnético altera a estrutura magnética do sistema, alinhando os spins da rede em sua direção. Para compreender o efeito do campo é suficiente resolver o hamiltoniano semi-clássico para esse sistema. Esse hamiltoniano é composto por um termo de exchange entre os spins da rede, um termo de interação dos spins da rede com o campo magnético e um termo referente à anisotropia magnética.

A interação entre um par de spins pode ser formulada pelo hamiltoniano de Heisenberg, definido na equação (2.27) como

$$
\mathcal{H}_{x}=-2 \sum_{i<j} J_{i j} \boldsymbol{S}_{i} \cdot \boldsymbol{S}_{j}=-\sum_{i, j} J_{i j} \boldsymbol{S}_{i} \cdot \boldsymbol{S}_{j}
$$

onde $J_{i j}$ é a constante de interação entre os spins $\boldsymbol{S}_{i}$ e $\boldsymbol{S}_{j}$.

No hamiltoniano de exchange do EuTe serão consideradas apenas interações entre os spins de cada íon $\mathrm{Eu}^{2+}$ com seus primeiros e segundos vizinhos. No ordenamento AFMII, cada sítio $\mathrm{Eu}^{2+}$ da rede, de spin $\boldsymbol{S}_{1}$, interage por exchange com uma constante $J_{1}$ com os primeiros vizinhos e com uma constante $J_{2}$ com os segundos vizinhos. Como vimos na figura 2.5, seis dos doze primeiros vizinhos encontram-se na mesma metade da rede, de spin $S_{1}$ e os outros seis encontram-se na outra metade da rede, de spin $S_{2}$. Todos os seis segundos vizinhos estão na metade oposta da rede, de spin $\boldsymbol{S}_{2}$. 
Para um sítio $\mathrm{Eu}^{2+}$ contido na metade da rede de spin $\boldsymbol{S}_{1}$, a soma de todas as interações de exchange resulta no seguinte hamiltoniano de exchange

$$
\mathcal{H}_{x}=6 J_{1} \boldsymbol{S}_{1} \cdot \boldsymbol{S}_{1}+6 J_{1} \boldsymbol{S}_{1} \cdot \boldsymbol{S}_{2}+6 J_{2} \boldsymbol{S}_{1} \cdot \boldsymbol{S}_{2}=-6 J_{1} S^{2}-6\left(J_{1}+J_{2}\right) \boldsymbol{S}_{1} \cdot \boldsymbol{S}_{2}
$$

A orientação dos spins em cada metade FM da rede AFM pode ser descrita em termos de suas respectivas magnetizações, através da relação $\boldsymbol{M}=N \boldsymbol{m u}$, onde $\boldsymbol{\mu}=$ $g \mu_{B} S$ e $N$ são respectivamente o momento magnético e a densidade dos átomos de európio na rede. Utilizando esta descrição

$$
S_{1} \rightarrow \frac{2 M_{1}}{N g \mu_{B}} \quad ; \quad S_{2} \rightarrow \frac{2 M_{2}}{N g \mu_{B}}
$$

onde $M_{1}$ e $M_{2}$ são as magnetizações de cada metade da rede. Substituindo (2.175) no hamiltoniano de exchange (2.174) obtemos

$$
\mathcal{H}_{x}=-24 J_{1} \frac{M^{2}}{N^{2} g^{2} \mu_{B}^{2}}-\frac{24}{N^{2} g^{2} \mu_{B}^{2}}\left(J_{1}+J_{2}\right) M_{1} \cdot M_{2}
$$

A constante de exchange $J_{2}$ é negativa e de magnitude superior a $J_{1}$ para o EuTe, de forma que o termo $\left(J_{1}+J_{2}\right) \rightarrow-\left|J_{1}+J_{2}\right|$, portanto podemos reescrever (2.176) como

$$
\mathcal{H}_{x}=-24 J_{1} \frac{M^{2}}{N^{2} g^{2} \mu_{B}^{2}}+\frac{24\left|J_{1}+J_{2}\right|}{N^{2} g^{2} \mu_{B}^{2}} M_{1} \cdot M_{2}
$$

As magnetizações $M_{1}$ e $M_{2}$ tem a mesma magnitude, sendo diferente apenas em suas orientações, que em campo zero são opostas, assim podemos substituir $M_{1}=M \hat{M}_{1}$ e $M_{2}=M \hat{M}_{2}$ onde $M$ é a magnetização de saturação de cada sub-rede e $\hat{M}_{1}$ e $\hat{M}_{2}$ são vetores unitários que apontam a direção das magnetizações de cada metade da rede. Com isso obtemos

$$
\mathcal{H}_{x}=-24 J_{1} \frac{M^{2}}{N^{2} g^{2} \mu_{B}^{2}}+\frac{24\left|J_{1}+J_{2}\right|}{N^{2} g^{2} \mu_{B}^{2}} M^{2} \hat{M}_{1} \cdot \hat{M}_{2}
$$

A anisotropia magnética do EuTe pode ser descrita por um hamiltoniano de dois termos $[68,66]$. Um deles caracterizado por uma constante de anisotropia $K_{1}$, é atribuído à interação de dipolo magnético e faz com que os spins fiquem contidos nos planos FM, formando os domínios T. O outro termo, de origem fenomenológica é caracterizado por uma constante $K_{2}$ e faz com que os spins orientem-se nas três direções equivalentes em 
energia que formam os domínios S. Num referencial no qual o eixo $z$ é paralelo à direção cristalina [111] e o eixo y é paralelo à direção [112̄], o hamiltoniano de anisotropia para cada sítio é

$$
\mathcal{H}_{a}=\frac{3 K_{1}}{8}\left(\cos \theta_{1}-\cos \theta_{2}\right)^{2}-\frac{K_{2}}{2}\left(\sin ^{2} 3 \varphi_{1}+\sin ^{2} 3 \varphi_{2}\right)
$$

onde $\theta_{i}$ e $\varphi_{i}$ são as coordenadas esféricas polar e azimutal, respectivamente, do vetor magnetização de cada uma das duas metades FM da rede.

A interação Zeeman dos spins da rede com o campo é descrita por um hamiltoniano do tipo

$$
\mathcal{H}_{\mathrm{Z}}=-\boldsymbol{\mu} \cdot \boldsymbol{B}
$$

onde $\boldsymbol{\mu}$ é o momento magnético de cada sítio. Em termos das magnetizações o hamiltoniano Zeeman (2.180) pode ser escrito como

$$
\mathcal{H}_{Z}=-\frac{2 M}{N}\left(\hat{M}_{1}+\hat{M}_{2}\right) \cdot \boldsymbol{B}
$$

Dessa forma o hamiltoniano total de cada spin da rede é dado pela soma dos termos referentes a cada interação

$$
\begin{array}{r}
\mathcal{H}_{t}=-24 J_{1} \frac{M^{2}}{N^{2} g^{2} \mu_{B}^{2}}+\frac{24\left|J_{1}+J_{2}\right|}{N^{2} g^{2} \mu_{B}^{2}} M^{2} \hat{M}_{1} \cdot \hat{M}_{2}- \\
-\frac{2 M}{N}\left(\hat{M}_{1}+\hat{M}_{2}\right) \cdot \boldsymbol{B}+\frac{3 K_{1}}{8}\left(\cos \theta_{1}-\cos \theta_{2}\right)^{2}-\frac{K_{2}}{2}\left(\sin ^{2} 3 \varphi_{1}+\sin ^{2} 3 \varphi_{2}\right)
\end{array}
$$

Para obtermos o hamiltoniano total do sistema, $\mathcal{H}_{T}$, multiplicamos $\mathcal{H}_{t}$ pela densidade de sítios $N$, para obtermos

$$
\begin{array}{r}
\mathcal{H}_{T}=-24 J_{1} \frac{M^{2}}{N g^{2} \mu_{B}^{2}}+\frac{24\left|J_{1}+J_{2}\right|}{N g^{2} \mu_{B}^{2}} M^{2} \hat{M}_{1} \cdot \hat{M}_{2}- \\
-2 M\left(\hat{M}_{1}+\hat{M}_{2}\right) \cdot \boldsymbol{B}+N \frac{3 K_{1}}{8}\left(\cos \theta_{1}-\cos \theta_{2}\right)^{2}-N \frac{K_{2}}{2}\left(\sin ^{2} 3 \varphi_{1}+\sin ^{2} 3 \varphi_{2}\right)
\end{array}
$$

Em campo zero, o mínimo de energia de $\mathcal{H}_{T}=\mathcal{H}_{x}+\mathcal{H}_{a}$ determina a orientação entre os vetores unitários $\hat{M}_{1}$ e $\hat{M}_{2}$, os quais caracterizam a ordem magnética do sistema. Para o termo de exchange, identifica-se o mínimo quando o produto escalar resulta -1 . Para isso,

$$
\hat{M}_{1}=-\hat{M}_{2}
$$


o que implica que

$$
\varphi_{1}=\varphi_{2} \text { e } \quad \theta_{1}=-\theta_{2}
$$

Esse resultado garante um arranjo AFM da rede.

Para o termo de anisotropia, quando $K_{1}$ e $K_{2}$ são positivos, e o são $\left(K_{1}=2.4 \times 10^{5} \mathrm{Jm}^{-3}\right.$ e $K_{2}=40.1 \mathrm{Jm}^{-3}$ segundo a referência [66] ), o termo de anisotropia terá seu valor mínimo quando o termo que depende de $\theta_{i}$ for mínimo e o módulo do termo que depende de $\varphi_{i}$ for máximo. O termo que depende de $\theta_{i}$ é mínimo quando $\cos \left(\theta_{1}\right)=\cos \left(\theta_{2}\right)$, o seja, quando os spins de cada metade da rede apontam em direções opostas e estão contidos em planos paralelos. O termo que depende de $\varphi_{i}$ tem módulo máximo quando $\sin \left(\varphi_{1}\right)=\sin \left(\varphi_{2}\right)=1$, resultando em $\varphi_{i}=\frac{\pi}{6}+\frac{n \pi}{3}, \operatorname{com} n=(0,1,2)$ para $0 \leq \varphi_{i} \leq \pi$. Dessa maneira foram obtidas três direções para as magnetizações da rede, as quais resultam em energias equivalentes para o sistema, de forma que o hamiltoniano em campo zero prevê três direções preferencias para os spins dentro dos planos paralelos.

Os planos em que estão contidos os spins são uma são a família de planos paralelos entre si e todos perpendiculares à direção [111], ou a uma das três outras diagonais da cúbica. Para cada uma das quatro diagonais haverá um domínio magnético diferente, totalizando quatro domínios equivalentes em energia chamados domínios $\mathrm{T}$, os quais são ilustrados na figura 2.7 .
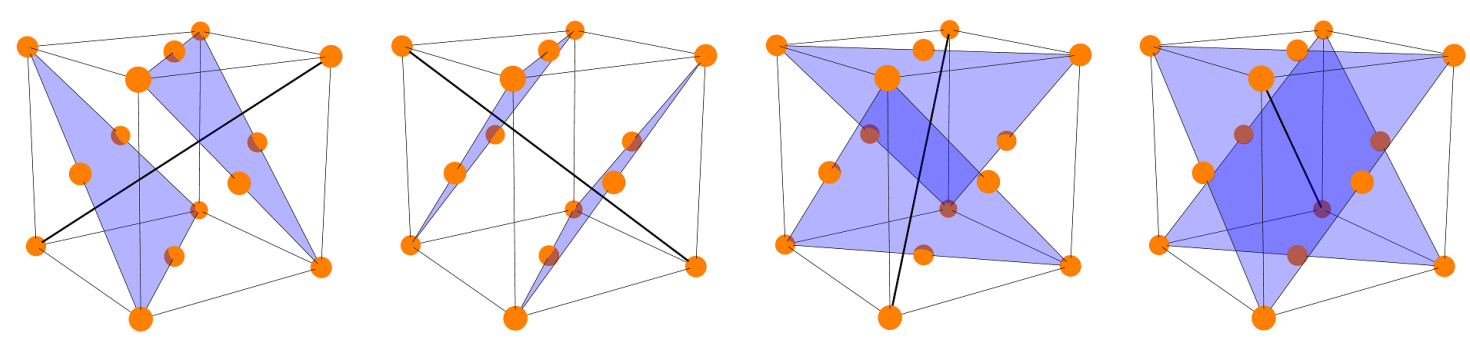

Figura 2.7: Domínios magnéticos $\mathrm{T}$ do EuTe. Podemos observar na figura os planos (111) e seus equivalentes, ( $(\overline{1} 1),(1 \overline{1} 1)$ e (11 $\overline{1})$ respectivamente.

Em cada domínio T, o mínimo de energia da equação (2.179) prevê para as coordenadas $\varphi_{i}$ três soluções distintas, que são energeticamente equivalentes, de forma a que existem três direções preferenciais para os spins no plano, os chamados domínios $S$, 
mostrados na figura 2.8. Para cada domínio T, existem três domínios $\mathrm{S}$, de forma que para o EuTe existem doze domínios magnéticos distintos de mesma energia. Em $T=0$ K espera-se que todos os doze domínios ocorram com a mesma probabilidade em um cristal real, entretanto num filme epitaxial fino, pode haver uma supressão de um ou mais domínios T, reduzindo esse número [69].

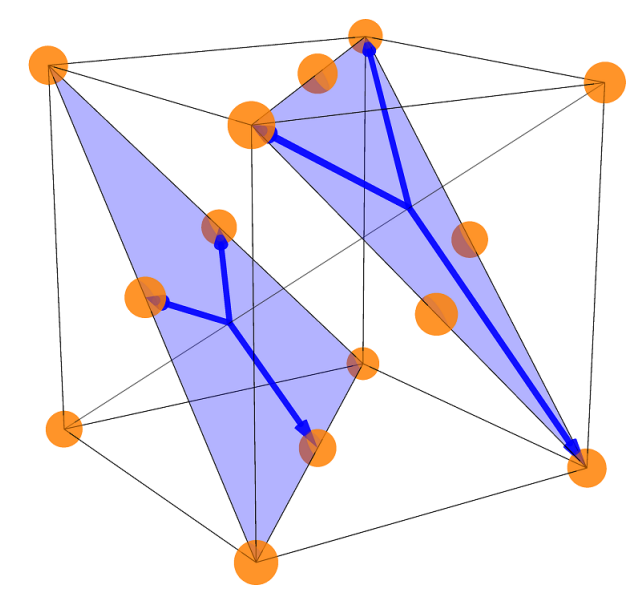

Figura 2.8: Domínios S. As setas em azul indicam as três direções preferenciais para os spins em um dado domínio T.

Quando aplica-se um campo magnético externo, cada domínio responde de uma maneira diferente, até a transição spin-flop, que para o EuTe ocorre para uma magnitude de campo magnético de $B_{s f} \sim 0.08 \mathrm{~T}$, dependendo do domínio [66]. Nessa intensidade de campo, as direções das magnetizações de todos os domínios realinham-se para uma direção perpendicular à direção do campo. Assim, a partir de $B_{s f}$, o EuTe apresenta um único domínio T e diz-se que o sistema encontra-se na fase spin-flop (SF), onde o aumento do campo causa uma redução do ângulo entre as magnetizações da cada metade da rede e a direção do campo, que vai de $\pi$ radianos em $B=B_{s f}$ a zero em $B=B_{S A T}$. Para o EuTe, o campo de saturação, a partir do qual a a rede está completamente alinhada com o campo vale $B_{S A T}=7.2$ T $[67,19]$. Para um filme epitaxial de EuTe sob ação de um campo aplicado na direção perpendicular ao plano epitaxial, o campo de desmagnetização faz com que o campo interno de 7.2 T seja atingido para uma magnitude do campo aplicado de $8.3 \mathrm{~T}$ [24]. 


\section{Capítulo 3}

\section{Materiais e métodos}

\subsection{Amostras}

As amostras de EuTe epitaxial (figura 3.1) foram fabricadas nas dependências do Laboratório Associado de Sensores e Materiais, no Instituto Nacional de Pesquisas Espaciais, São José dos Campos, SP, pelos pesquisadores Eduardo Abramof, Paulo Rappl e Beatriz Díaz. Foram estudadas amostras com camadas epitaxiais de diversas espessuras, determinadas pelos fabricantes através da difração de raios- $x$, crescidas na direção $[1,1,1]$ em substratos de $\mathrm{BaF}_{2}[1,1,1]$. Uma descrição mais detalhada das técnicas de crescimento e caracterização pode ser obtida na referência [70].

As amostras epitaxiais de EuSe foram fornecidas pelo grupo do Prof. Günther Bauer, sediado no Institut für Halbleiter und Festkörperphysik, Johannes Kepler Universität, em Linz, Áustria.

Foram realizados experimentos de espectroscopia de absorção em um filme epitaxial de $0.34 \mu m$ de EuSe depositado na direção $[1,1,1]$ sobre substrato de $\mathrm{BaF}_{2}[1,1,1]$, cuja diferença de parâmetro de rede é menor do que $0.1 \%$, o que permite cristais de boa qualidade estrutural. Os detalhes do crescimento e caracterização dessas amostras podem ser encontrados na referência [71]. 


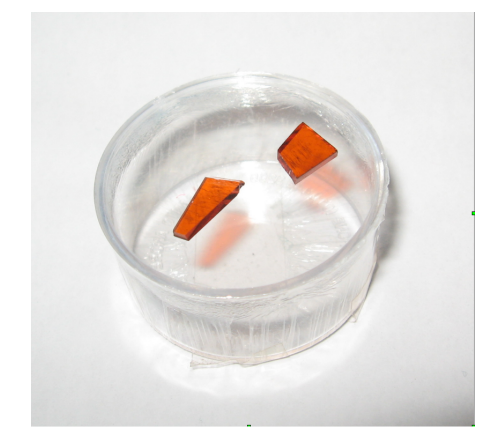

Figura 3.1: Amostras epitaxiais de EuTe crescidas em substrato de $\mathrm{BaF}_{2}$.

\subsection{Arranjo experimental}

O arranjo experimental para a espectroscopia de absorção e emissão consiste de um criostato CRYOGENIC resfriado com hélio líquido (LHe), o qual opera em temperaturas entre $1.5 \mathrm{~K}$ e $300 \mathrm{~K}$ na região da amostra, com uma precisão de $0.001 \mathrm{~K}$. A temperatura é controlada com um controlador LakeShore 340, que utiliza sensores de temperatura CERNOX calibrados e posicionados no interior do criostato e nos porta-amostras. O criostato é equipado com uma bobina supercondutora, resfriada a LHe, que fornece um campo magnético uniforme na região da amostra de até $9.5 \mathrm{~T}$, o qual pode ter seu sentido invertido por comando da fonte de alimentação CRYOGENIC. A amostra fica no interior do criostato, posicionada em porta-amostras desenvolvidos especialmente para cada medida. A seguir serão especificados os sistemas utilizados para as medidas de absorção e de fotoluminescência.

\subsubsection{Montagem para absorção}

Os espectros de absorção são obtidos a partir de medidas da transmitância da amostra em função do comprimento de onda da luz incidente, utilizando-se a montagem da figura 3.2. A fonte de luz utilizada é uma lâmpada de xenônio CAIRN de alto desempenho, com potência nominal de $60 \mathrm{~W}$, acoplada a um espectrômetro de rede de difração do modelo SPEX 270-M, em detalhe na figura 3.3, que funciona como um monocromador, onde o comprimento de onda da luz na saída pode ser controlado pelo 
computador.

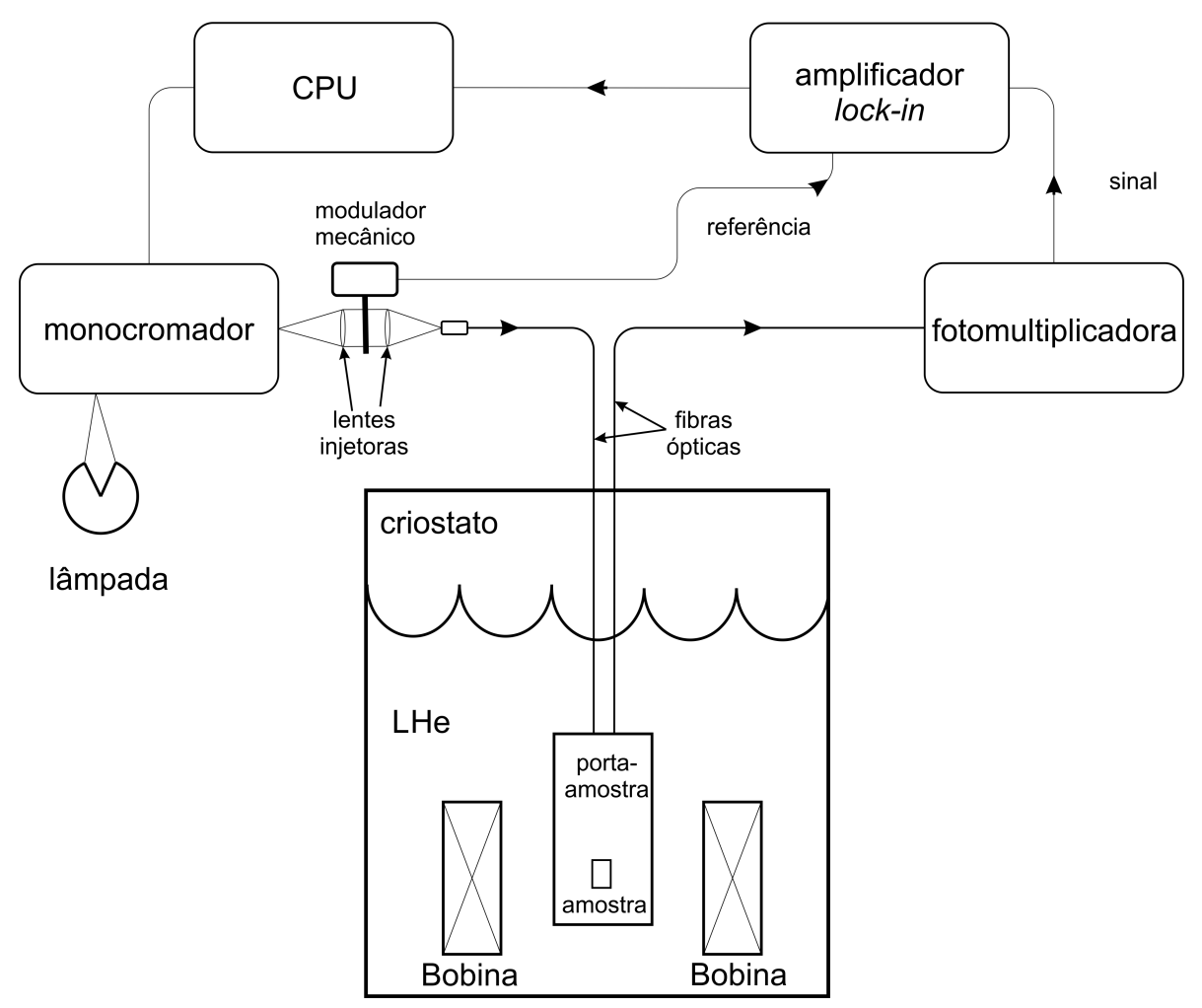

Figura 3.2: Arranjo experimental para medidas do espectro de absorção.

A luz monocromática atravessa um modulador mecânico de frequência ajustável e é injetada numa fibra óptica através de uma lente objetiva Newport. A fibra óptica é acoplada ao porta-amostra, que é inserido no interior do criostato. Esse porta-amostra, apresentado em detalhe na figura 3.4, foi idealizado e montado no Laboratório de Magneto-Óptica e Espectroscopia Não-Linear do IFUSP. Como podemos ver na figura 3.4, a luz que provém da fibra óptica é colimada por uma lente GRIN e incidida sobre um par de espelhos finamente ajustáveis, que conduzem o feixe de luz no interior do portaamostra. Ali o feixe atravessa um polarizador circular, a amostra e então é injetado na fibra coletora, que leva o sinal luminoso para fora do criostato. O polarizador circular é construído pela justaposição de um polarizador linear CORNING de alto desempenho, e uma placa de $1 / 4$ de onda. O eixo rápido da placa de onda não é identificado pelo fabricante e para identificá-lo foi utilizada uma técnica baseada numa simplificação do procedimento descrito na referência [72], a qual foi apresentada em conferência 


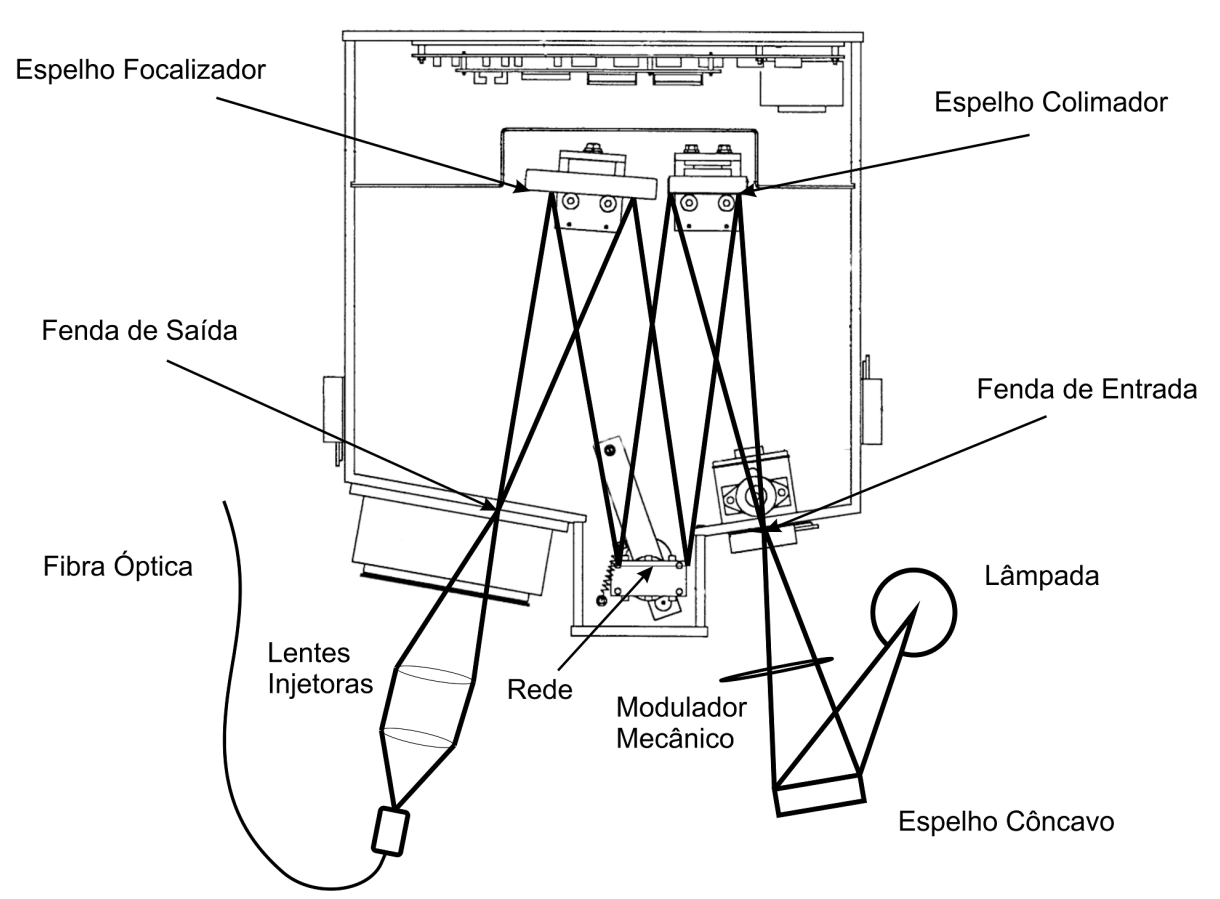

Figura 3.3: Monocromador SPEX 270-M

nacional como trabalho de Iniciação Científica [73].

A luz emergente da amostra pode ter intensidade muito baixa, dependendo da espessura e coeficiente de absorção da amostra. Por isso a deteç̧ão é feita com uma fotomultiplicadora, a qual é acoplada a um amplificador de lock-in Stanford, sintonizado na frequência de referência recebida do modulador mecânico. O sinal do lock-in em função do comprimento de onda da luz incidente é registrado no sistema de aquisição, que fornece assim o espectro de transmissão do material. Para calcular o espectro de absorção a partir do espectro de transmissão, um sinal de fundo é registrado, seguindo exatamente o mesmo procedimento de aquisição, porém sem nenhuma amostra no porta-amostra. A subtração desse espectro de fundo corrige eventuais ruídos que estiverem presentes no sinal, como por exemplo a fotoluminescência das fibras ópticas. Essa subtração é feita de maneira a levar em conta as eventuais fugas de luz do sistema, que entram no cálculo como parâmetros ajustáveis. 


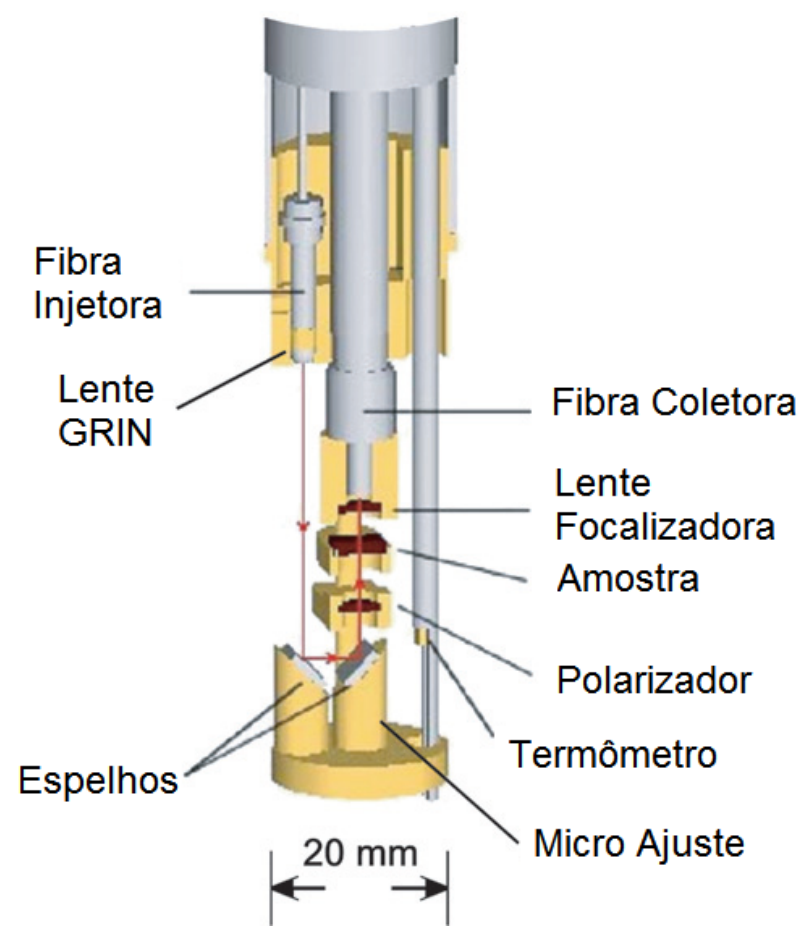

Figura 3.4: Detalhe do porta-amostras utilizado na espectroscopia de absorção.

\subsubsection{Montagem para fotoluminescência}

Na espectroscopia de fotoluminescência, utiliza-se a montagem da figura 3.5. A fonte de luz utilizada foi um laser monocromático de Nd:YAG da marca Cobolt, de comprimento de onda nominal $532 \mathrm{~nm}$, cuja potência, de até $50 \mathrm{~mW}$, pode ser finamente ajustada através de uma combinação de filtros de densidade neutra da Newport. A luz do laser é injetada em uma fibra óptica com uma lente objetiva Newport e conduzida até o porta-amostra, que é inserido no criostato. Esse porta-amostra, ilustrado em detalhe na figura 3.6, foi idealizado e construído no Laboratório de Magneto-Óptica e Espectroscopia Não-Linear do IFUSP, especialmente para medidas de fotoluminescência e fotoluminescência de excitação (bastando mudar a fonte de luz para o monocromador da figura 3.3), ambas com a possibilidade de controlar a de polarização da luz. A luz do laser que chega pela fibra óptica, passa por um polarizador linear CORNING e por um conjunto de duas lentes ajustáveis, uma colimadora e uma focalizadora, que concentram o feixe luminoso num disco de $\sim 0.5 \mathrm{~mm}^{2}$ na superfície da amostra. A luz 


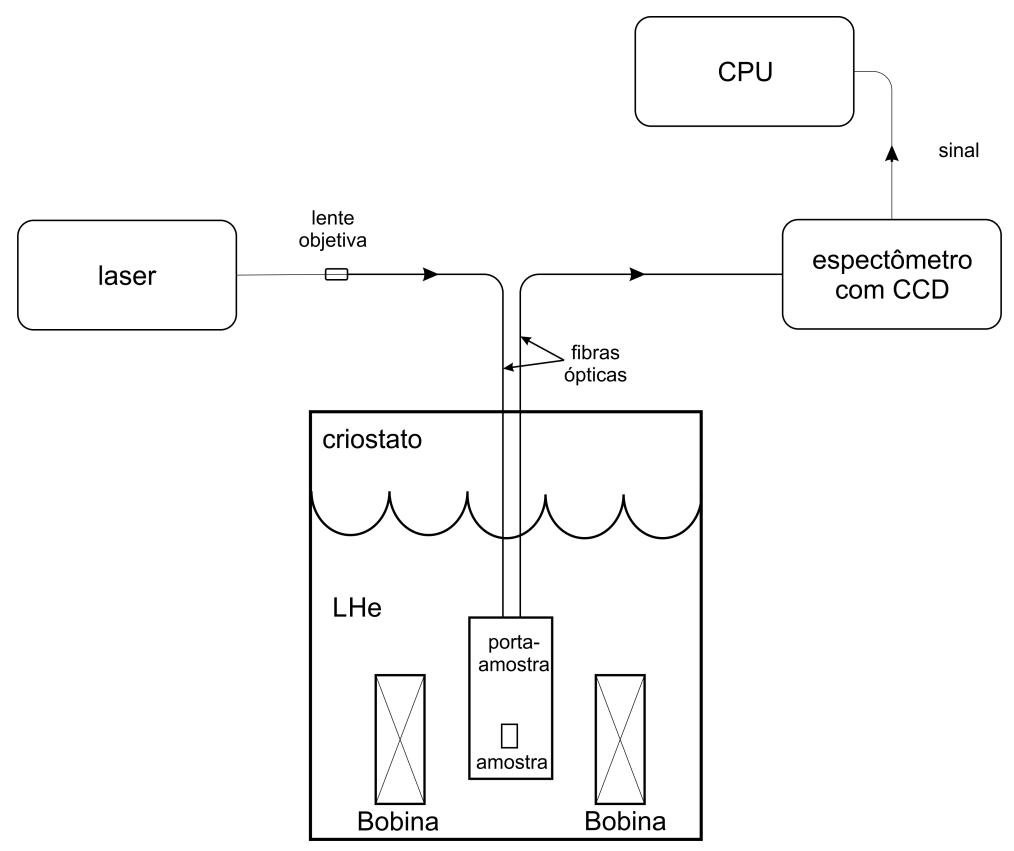

Figura 3.5: Arranjo experimental para medidas de fotoluminescência.

emitida pela amostra é coletada por outra fibra óptica, que conduz o sinal luminoso até o sistema de detecção.

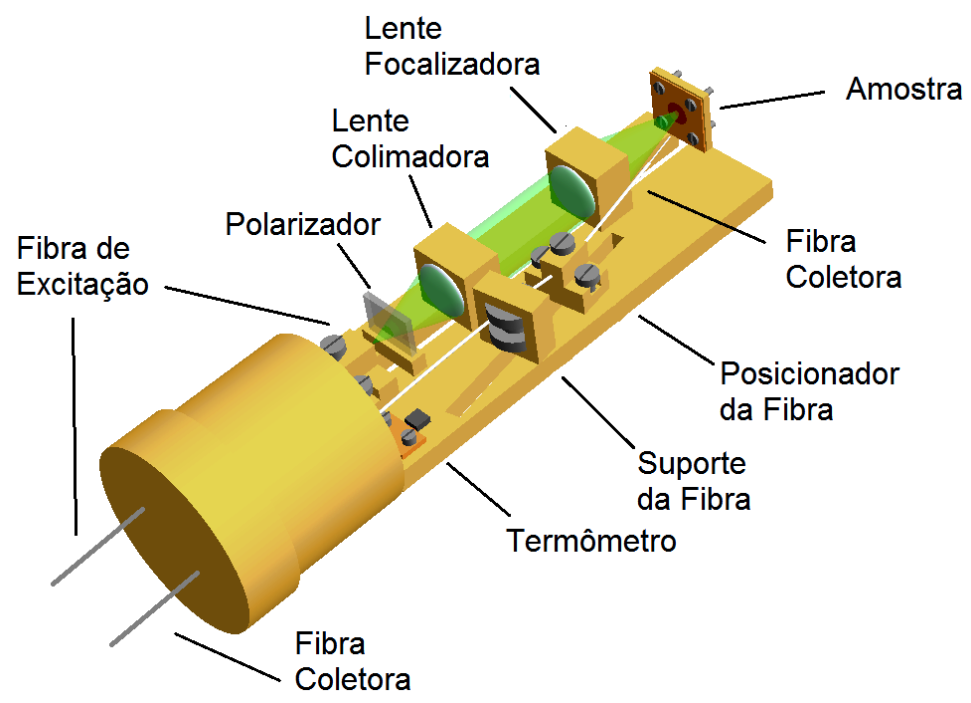

Figura 3.6: Detalhe do porta-amostras utilizado na espectroscopia de PL.

O detector é constituído de um espectrômetro do modelo TRIAX 550, equipado com um dispositivo de carga acoplada (CCD) resfriado a $\mathrm{N}_{2}$ líquido. Como pode ser visto 
na figura 3.7, na entrada do espectrômetro é colocado um filtro que bloqueia a radiação do laser refletida pela amostra, evitando que a luz do laser interfira no espectro de luminescência do material. Dentro do espectrômetro, a luz proveniente da amostra é separada pela rede de difração e direcionada através de dois espelhos para o CCD, onde a intensidade de cada frequência é capturada e registrada no sistema de aquisição, que fornece diretamente o espectro de emissão da amostra. Esse sistema é capaz de detectar a intensidade da luminescência com alta resolução em um intervalo de até $75 \mathrm{~nm}$ em uma mesma medida.

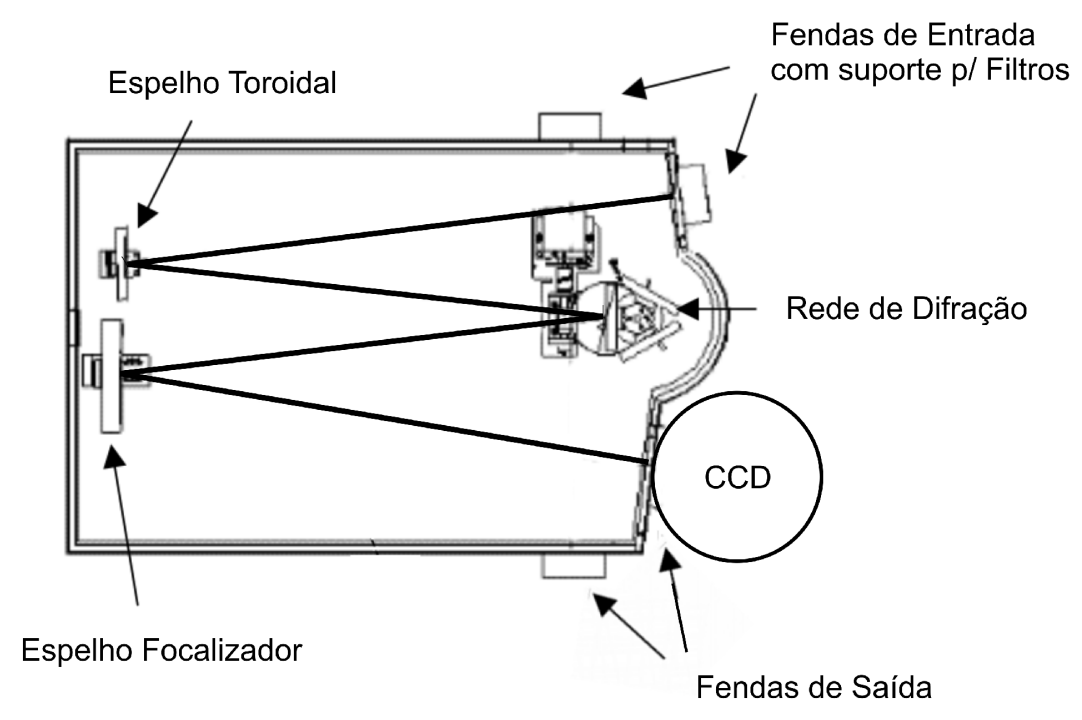

Figura 3.7: Espectrômetro TRIAX 550, equipado com um CCD

Para medidas da fotoluminescência de excitação, utilizamos a mesma montagem, com exceção da fonte de luz, que é substituída pelo monocromador da figura 3.3, para que seja possível variar o comprimento de onda da luz de excitação.

A seguir, apresentamos fotografias do porta-amostra para espectroscopia de absorção (figura 3.8), do porta-amostra para espectroscopia de PL e PLE (figura 3.9) e do criostato (figura 3.10). 


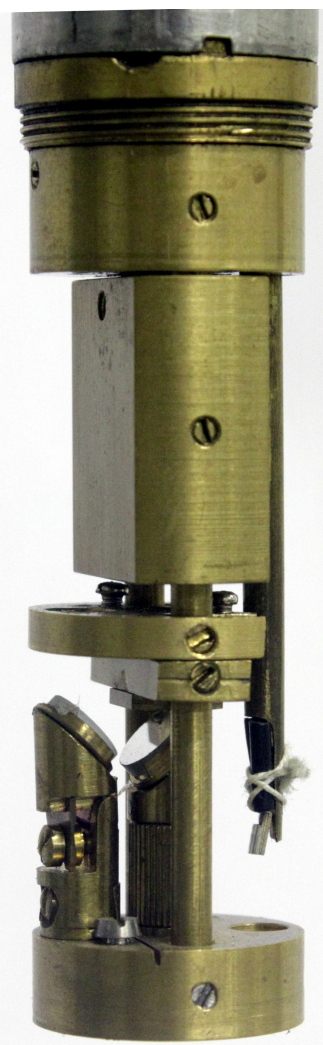

Figura 3.8: Porta-amostra utilizado na espectroscopia de absorção.

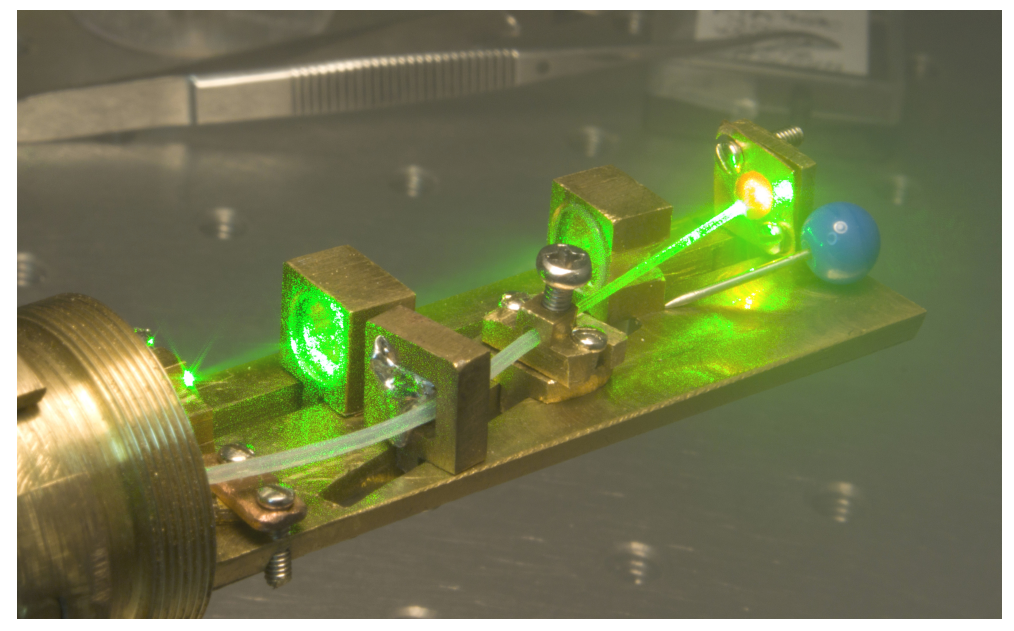

Figura 3.9: Porta-amostra utilizado na espectroscopia de PL e PLE. 


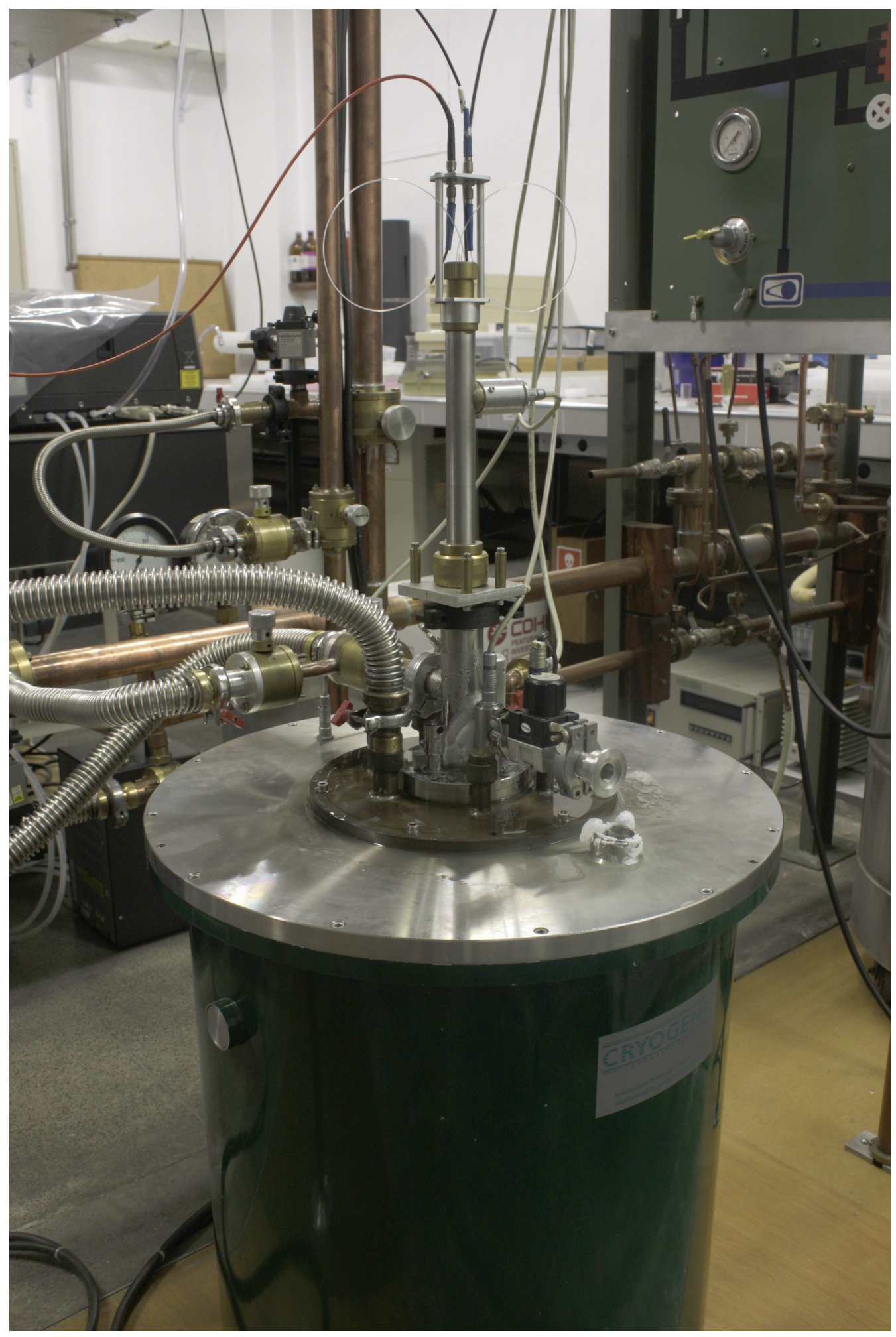

Figura 3.10: Criostato CRYOGENIC, com acesso óptico dado pelas das fibras ópticas acopladas aos porta-amostras. 


\section{Capítulo 4}

\section{Resultados}

\subsection{Modelo para a absorção óptica nos EuX}

Os EuX são semicondutores magnéticos que apresentam uma faixa de transparência na região visível do espectro eletromagnético. A partir de certa energia da radiação incidente, os EuX tornam-se absorventes, em virtude da excitação de estados eletrônicos através da luz. Esse comportamento é típico em semicondutor: os estados eletrônicos de valência encontram-se totalmente populados e o primeiro estado excitado é o fundo da banda de condução, separado da banda de valência por uma faixa de energias proibidas, o gap de energia, $E_{G}$. Quando um elétron na banda de valência recebe energia igual ou maior do que $E_{G}$, o mesmo pode absorver essa energia e saltar para a banda de condução, passando para um estado não-localizado.

A incidência de fótons sobre um semicondutor pode desencadear esse processo, onde os elétrons da banda de valência podem absorver um fóton saltando para a banda de condução. O material pode absorver fótons com energias proporcionais à densidade de estados de condução. A grandeza que caracteriza a probabilidade de absorção para cada energia dos fótons de excitação é o coeficiente de absorção do material $\alpha(\hbar \omega)$.

Nesta seção será formulado um modelo para o coeficiente de absorção dos EuX. A absorção de fótons com energias próximas do gap nos EuX é governada pela transição de elétrons da camada de valência $4 f$ dos íons $\mathrm{Eu}^{2+}$ para o fundo da banda de condução, 
composta por estados eletrônicos $5 d\left(t_{2 g}\right)$ [19]. Para obter-se o coeficiente de absorção será calculada a probabilidade de transição entre os estados $4 f$ e $5 d\left(t_{2 g}\right)$ em função da energia dos fótons de excitação, segundo o formalismo da mecânica quântica de átomos e moléculas. O modelo da mecânica quântica para a absorção de luz consiste na transição de um elétron do estado fundamental para um estado excitado de energia superior através da absorção de fótons. Como os níveis atômicos são quantizados, existem faixas de energia que não permitem absorção, caracterizando uma transparência do material para radiação com essa faixa de energia. Se a energia da radiação incidente é suficiente para levar um elétron para um estado excitado, acontece a absorção de luz, que pode ser detectada por um decréscimo na intensidade da luz que passou pelo material em relação à intensidade da luz incidente.

Antes de mais nada serão apresentados os conceitos fundamentais utilizados no modelo: a propagação de ondas em meios absorventes, a polarização da luz, e a absorção óptica em átomos de um ou mais elétrons, onde será derivada a probabilidade de transição entre dois estados eletrônicos quaisquer. Em seguida será apresentado o modelo aceito atualmente para os níveis de energia dos EuX e finalmente será calculado o coeficiente de absorção dos EuX, para diferentes polarizações da luz, levando em consideração ainda a influência da aplicação de um campo magnético externo.

\subsubsection{Propagação de ondas eletromagnéticas em um dielétrico}

Na teoria clássica do eletromagnetismo, uma onda luminosa é descrita por um campo elétrico e um campo magnético que propagam-se no espaço, os quais são definidos pelas equações de Maxwell, que no vácuo são

$$
\begin{aligned}
\nabla \cdot \boldsymbol{E} & =0 \\
\nabla \cdot \boldsymbol{B} & =0 \\
\nabla \times \boldsymbol{E} & =-\frac{\partial \boldsymbol{B}}{\partial t} \\
\nabla \times \boldsymbol{B} & =\mu_{0} \epsilon_{0} \frac{\partial \boldsymbol{E}}{\partial t}
\end{aligned}
$$


Tomando-se o rotacional de (4.3) e (4.4) e lançando mão da identidade vetorial

$$
\nabla \times(\nabla \times A)=\nabla(\nabla \cdot A)-\nabla^{2} A
$$

obtemos as equações de onda para os campos elétrico e magnético

$$
\begin{aligned}
& \nabla^{2} \boldsymbol{E}=\mu_{0} \epsilon_{0} \frac{\partial^{2} \boldsymbol{E}}{\partial t^{2}} \\
& \nabla^{2} \boldsymbol{B}=\mu_{0} \epsilon_{0} \frac{\partial^{2} \boldsymbol{B}}{\partial t^{2}}
\end{aligned}
$$

onde foram utilizadas as equações (4.1) e (4.2) que estabelecem que as divergências de $\boldsymbol{E}$ e $\boldsymbol{B}$ se anulam.

As equações (4.6) e (4.7) admitem soluções na forma de ondas planas progressivas, as quais definindo a direção de propagação da luz como a direção $\hat{z}$, são dadas por

$$
\begin{aligned}
& \boldsymbol{E}=\hat{\boldsymbol{\xi}}_{E} E_{0} \cos (k z-\omega t) \\
& \boldsymbol{B}=\hat{\boldsymbol{\xi}}_{B} B_{0} \cos (k z-\omega t)
\end{aligned}
$$

onde $c=\frac{\omega}{k}=\frac{1}{\sqrt{\mu_{0} \epsilon_{0}}}$ é a velocidade de propagação e $\hat{\boldsymbol{\xi}}_{E}$ e $\hat{\boldsymbol{\xi}}_{B}$ são vetores unitários que obedecem as equações de Maxwell.

Substituindo (4.8) e (4.9) nas equações (4.1) e (4.2), respectivamente, vemos que as componentes de $\boldsymbol{E}$ e $\boldsymbol{B}$ na direção $\hat{z}$ têm de ser nulas, portanto

$$
\begin{gathered}
\hat{\boldsymbol{\xi}}_{E} \perp \boldsymbol{k} \\
\hat{\boldsymbol{\xi}}_{B} \perp \boldsymbol{k}
\end{gathered}
$$

o que mostra que $\boldsymbol{E}$ e $\boldsymbol{B}$ são ondas planas.

Além disso, utilizando a equação (4.3), obtemos

$$
\boldsymbol{\nabla} \times \boldsymbol{E}=-\frac{\partial \boldsymbol{B}}{\partial t} \rightarrow\left\{\begin{array}{l}
-k\left(E_{0}\right)_{y}=\omega\left(B_{0}\right)_{x} \\
-k\left(E_{0}\right)_{x}=\omega\left(B_{0}\right)_{y}
\end{array}\right.
$$

ou, de maneira mais compacta,

$$
\hat{\boldsymbol{\xi}}_{B} B_{0}=\frac{k}{\omega} \hat{\boldsymbol{z}} \times \hat{\boldsymbol{\xi}}_{E} E_{0}
$$


de onde podemos ver que $\hat{\boldsymbol{\xi}}_{B}$ e $\hat{\boldsymbol{\xi}}_{E}$ são mutuamente perpendiculares.

$O$ vetor $\hat{\xi}_{E}$ usualmente é utilizado para definir a polarização da luz, o que será o tema da seção seguinte.

\subsubsection{Polarização da luz}

A polarização de uma onda eletromagnética é determinada pela direção do vetor campo elétrico associado a essa onda. Neste trabalho serão utilizados dois tipos de polarização da luz: a polarização linear e a polarização circular. Na luz linearmente polarizada, o vetor campo elétrico é fixo em uma direção perpendicular à direção de propagação. $\mathrm{Na}$ luz circularmente polarizada o vetor campo elétrico rotaciona ao redor da direção de propagação, mantendo-se sempre no plano perpendicular a essa direção. O vetor campo elétrico pode rotacionar no sentido horário ou anti-horário, para um observador situado na fonte de luz, olhando para o sentido de propagação. Nessa definição, a luz cujo vetor campo elétrico rotaciona no sentido horário é denominada RHCP, que é o acrônimo da expressão da língua inglesa Right Handed Circularly Polarized Light, pois segundo a definição adotada, a rotação desse feixe acompanha a supinação da mão direita, quando o dedo polegar aponta o sentido de propagação. Para a rotação do vetor campo elétrico no sentido anti-horário, vale o mesmo para a mão esquerda, portanto chama-se essa luz de LHCP, de Left Handed Circularly Polarized Light. A figura 4.1 resume essa notação.

É sempre necessário ser cauteloso com a definição adotada, pois uma confusão das notações pode levar a um resultado totalmente inverso. Em muitos textos como por exemplo Optics de E. Hecht [74], o nome que se dá à RHCP é LCP left-circular polarization e para LHCP dá-se o nome RCP, onde o autor define a polarização para a direita como sendo aquela na qual o vetor campo elétrico rotaciona no sentido horário para um observador olhando para a fonte e para a esquerda quando o vetor campo elétrico gira no sentido anti-horário. Neste trabalho usaremos sempre a notação LHCP e RHCP como definidas acima.

A figura 4.2 mostra o vetor campo elétrico de uma onda luminosa RHCP, cujo vetor 
LHCP

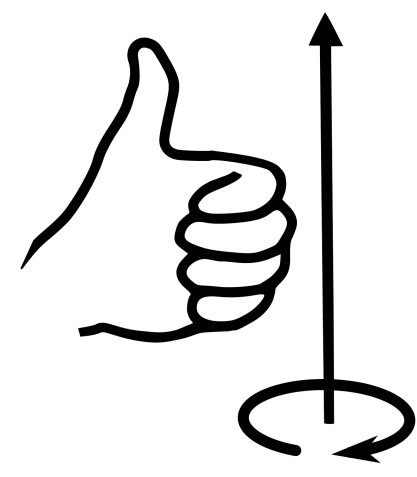

RHCP

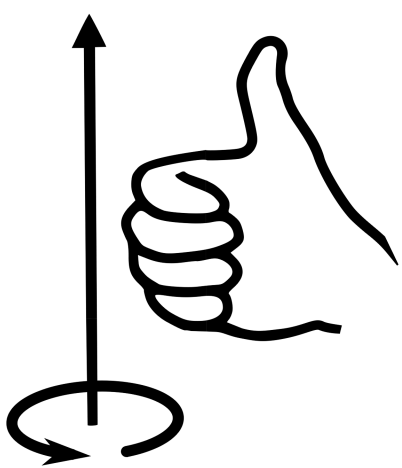

Fonte de luz

Figura 4.1: Notação utilizada para a luz circularmente polarizada. RHCP: $E(z, t)=x E_{0} \cos (k z-\omega t)-y E_{0} \operatorname{sen}(k z-\omega t)$

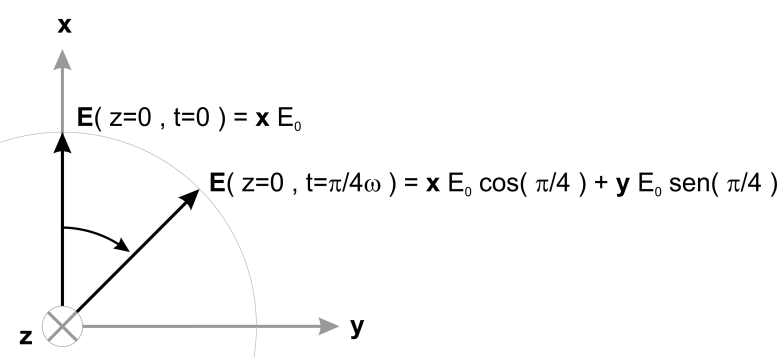

Figura 4.2: Vetor campo elétrico de uma onda de luz RHCP que se propaga na direção $z$ em dois instantes: $t=0$ e $t=\pi / 4 \omega$.

campo elétrico, no vácuo é dado por

$$
\boldsymbol{E}(z, t)=\hat{\boldsymbol{x}} E_{0} \cos (k z-\omega t)-\hat{\boldsymbol{y}} E_{0} \operatorname{sen}(k z-\omega t)=\operatorname{Re}\left[(\hat{\boldsymbol{x}}+\imath \hat{\boldsymbol{y}}) E_{0} e^{\imath(k z-\omega t)}\right]
$$

Nesse caso o vetor de polarização complexo é dado por $\tilde{\boldsymbol{\xi}}^{(R H C P)}=\hat{\boldsymbol{x}}+\imath \hat{\boldsymbol{y}}$. Analogamente para uma onda de luz LHCP, o vetor campo elétrico é

$$
\boldsymbol{E}(z, t)=\hat{\boldsymbol{x}} E_{0} \cos (k z-\omega t)+\hat{\boldsymbol{y}} E_{0} \operatorname{sen}(k z-\omega t)=\operatorname{Re}\left[(\hat{\boldsymbol{x}}-\imath \hat{\boldsymbol{y}}) E_{0} e^{\imath(k z-\omega t)}\right]
$$

de onde podemos concluir que $\tilde{\boldsymbol{\xi}}^{(L H C P)}=\hat{\boldsymbol{x}}-\imath \hat{\boldsymbol{y}}$. 
Para uma onda de luz linearmente polarizada, onde o campo elétrico oscila na direção $x$, temos

$$
\boldsymbol{E}(z, t)=\hat{\boldsymbol{x}} E_{0} \cos (k z-\omega t)=\operatorname{Re}\left[\hat{\boldsymbol{x}} E_{0} e^{\imath(k z-\omega t)}\right]
$$

de forma que o vetor de polarização é $\tilde{\boldsymbol{\xi}}^{\| x}=\hat{\boldsymbol{x}}$ e para uma onda onda o vetor campo elétrico oscila na direção $y$ o vetor de polarização é $\tilde{\boldsymbol{\xi}}^{\perp x}=\hat{\boldsymbol{y}}$.

Então uma onda de polarização qualquer pode ser descrita por um campo elétrico dado por

$$
\boldsymbol{E}(z, t)=\operatorname{Re}\left[\tilde{\boldsymbol{\xi}} E_{0} e^{\imath(k z-\omega t)}\right]
$$

onde $\tilde{\xi}$ é o vetor de polarização adequado à polarização desejada. Para que o vetor $\tilde{\xi}$ seja unitário, o mesmo deve ser normalizado. Para cada uma das polarizações utilizadas neste trabalho, normalizando os vetores de polarização encontrados acima, teremos os seguintes versores de polarização complexos

$$
\begin{aligned}
\hat{\boldsymbol{\xi}}^{(+)}=\frac{\hat{\boldsymbol{x}}+\imath \hat{\boldsymbol{y}}}{\sqrt{2}} & (\mathrm{RHCP}) \\
\hat{\boldsymbol{\xi}}^{(-)}=\frac{\hat{\boldsymbol{x}}-\imath \hat{\boldsymbol{y}}}{\sqrt{2}} & (\mathrm{LHCP}) \\
\hat{\boldsymbol{\xi}}^{(\|)}=\hat{\boldsymbol{x}} & \boldsymbol{E} \| x \\
\hat{\boldsymbol{\xi}}^{(\perp)}=\hat{\boldsymbol{y}} & \boldsymbol{E} \perp x
\end{aligned}
$$

\subsubsection{Absorção óptica por um material}

As ondas eletromagnéticas descritas na seção 4.1.1 carregam uma quantidade de energia armazenada nos campos elétrico e magnético, cuja densidade é dada por

$$
\begin{aligned}
& u_{E}=\frac{1}{2} \epsilon_{0} \boldsymbol{E}^{2} \\
& u_{B}=\frac{1}{2} \epsilon_{0} \boldsymbol{B}^{2}
\end{aligned}
$$

Ao incidirem sobre um material absorvente, parte dessa energia é absorvida pelo material. A redução na energia é proporcional à distância $z$ percorrida no material

$$
\Delta u=-\alpha u \Delta z
$$


onde a constante de proporcionalidade $\alpha$ é o coeficiente de absorção do material.

A equação (4.25) pode ser rearranjada para

$$
\frac{\Delta u}{u}=-\alpha \Delta z
$$

o que por integração resulta em

$$
\ln u-\ln u_{0}=-\alpha z
$$

ou

$$
u=u_{0} e^{-\alpha x}
$$

Mas $u_{E} \propto E_{0}^{2}$ e $u_{B} \propto B_{0}^{2}$, portanto podemos perceber que as amplitudes $E_{0}$ e $B_{0}$ são diminuídas por um fator $e^{-\frac{\alpha}{2} z}$, de forma que os campos elétrico e magnético num meio absorvente podem ser descritos por

$$
\begin{aligned}
& \boldsymbol{E}=\hat{\boldsymbol{\xi}}_{E} E_{0} e^{-\frac{\alpha z}{2}} \cos (k z-\omega t) \\
& \boldsymbol{B}=\hat{\boldsymbol{\xi}}_{B} B_{0} e^{-\frac{\alpha z}{2}} \cos (k z-\omega t)
\end{aligned}
$$

A absorção pelo meio material pode ser formulada em termos da intensidade do feixe de luz, que é definida como a média temporal do vetor de Poynting, que para um material não magnético, é definido como $S=E \times \frac{B}{\mu_{0}}$, portanto

$$
I \equiv\left|\left\langle E \times \frac{B}{\mu_{0}}\right\rangle\right|
$$

Substituindo as equações (4.29) e (4.30) em (4.31) obtemos

$$
I=\left|\hat{\boldsymbol{\xi}}_{E} \times \hat{\boldsymbol{\xi}}_{B}\right|\left\langle\frac{E_{0} B_{0}}{\mu_{0}} e^{-\alpha z} \cos ^{2}(k z-\omega t)\right\rangle=\frac{E_{0} B_{0}}{\mu_{0}} e^{-\alpha z}\left\langle\cos ^{2}(k z-\omega t)\right\rangle
$$

Da equação (4.14), podemos determinar a relação entre as amplitudes dos campos elétrico e magnético como

$$
B_{0}=\frac{k}{\omega} E_{0}=\frac{E_{0}}{c}
$$


Substituindo (4.33) em (4.32) e considerando que $\left\langle\cos ^{2}(k z-\omega t)\right\rangle=1 / 2$ obtemos

$$
I=\frac{E_{0}^{2}}{2 \mu_{0} c} e^{-\alpha z} \underbrace{=}_{c=\frac{1}{\sqrt{\mu_{0}} \epsilon_{0}}} \frac{1}{2} c \epsilon_{0} E_{0}^{2} e^{-\alpha z}
$$

Para uma onda que se propaga no vácuo, substituímos $\alpha=0$ em (4.34) para obtermos

$$
I_{0}=\frac{1}{2} c \epsilon_{0} E_{0}^{2}
$$

Ao incidir sobre um material absorvente, sua a intensidade $I_{0}$ será atenuada pelo fator $e^{-\alpha z}$, de forma que após percorrer uma distância $d$ no material, a intensidade da onda vale

$$
I=I_{0} e^{-\alpha d}
$$

Esse resultado é conhecido como a "Lei de Beer-Lambert", que está ilustrada na figura 4.3 .

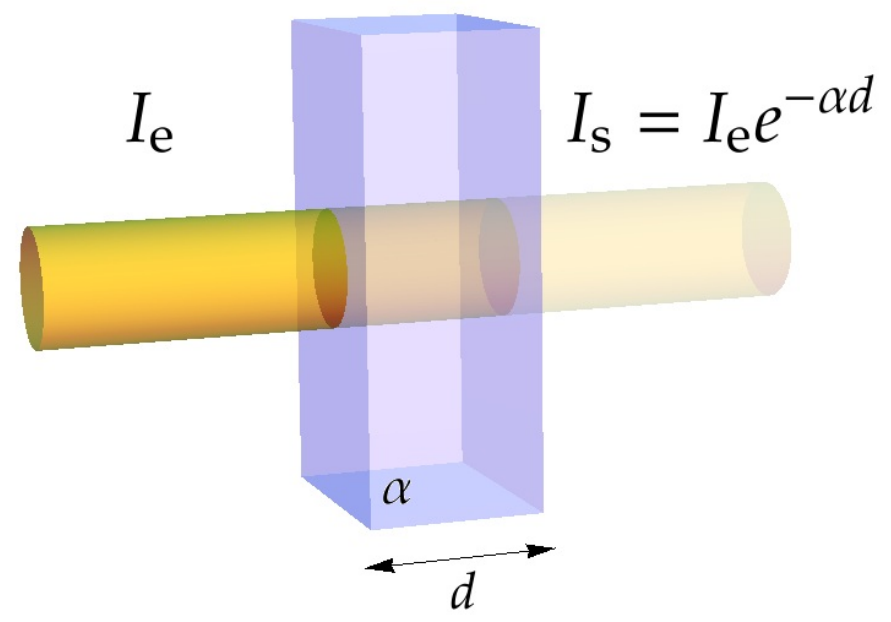

Figura 4.3: A Lei de Beer-Lambert para a absorção num dielétrico com coeficiente de absorção $\alpha$, no qual um feixe de intensidade inicial $I_{0}$ percorre uma distância $d$ no interior do dielétrico e tem sua intensidade atenuada.

A intensidade absorvida pelo material é dada pela intensidade inicial, menos a intensidade da onda após percorrer a distância $d$ no material

$$
I_{a b s}=I_{0}-I_{0} e^{-\alpha d}=I_{0}\left(1-e^{-\alpha d}\right)
$$


A seguir iremos estabelecer um modelo para o cálculo do coeficiente de absorção do material. Vamos supor que um feixe de luz monocromática com uma área de secção transversal $A$ atravessa uma espessura $d z$ de um dielétrico cristalino. Nesse processo, fótons podem ser absorvidos pelos sítios da rede segundo uma probabilidade $W$. O número total de fótons absorvidos no volume $A d z$ é dado pelo número de sítios absorventes no volume, $N$, o qual depende da estrutura química do material, multiplicado pela probabilidade $W$ de cada sítio absorver um fóton. No volume $A d z$, o número de fótons absorvidos vale

$$
N A d z \times W
$$

ou, por unidade de área,

$$
N d z \times W
$$

Multiplicando o número de fótons absorvidos pela energia de cada fóton, deve-se obter a intensidade perdida $d I$ pelo feixe ao atravessar a espessura $d z$ do material.

De acordo com a mecânica quântica, a energia de um fóton é determinada por sua frequência $v=\omega / 2 \pi$,

$$
E=\hbar \omega
$$

Multiplicando-se (4.39) por $\hbar \omega$ obtemos $d I=N d z W \hbar \omega$, ou

$$
\frac{d I}{d z}=N W \hbar \omega
$$

Por outro lado, a intensidade de luz absorvida por um material foi deduzida na equação (4.37) como

$$
I_{\mathrm{abs}}=I_{0}\left(1-e^{-\alpha z}\right)
$$

Diferenciando-se a equação (4.42) em relação a $z$, obtemos a energia perdida pelo feixe em uma espessura $d z$ como

$$
\frac{d I}{d z}=\alpha I_{0} e^{-\alpha z}
$$


Como $d z$ é tão pequeno quanto se queira, podemos considerar que a intensidade $I$ do feixe não sofreu atenuação significante ao percorrer um distância $d z$ no material $\left(e^{-\alpha d z}=1\right)$, de modo que

$$
\frac{d I}{d z}=\alpha I_{0}
$$

Ao compararmos a equação (4.44) com (4.41), encontramos o coeficiente de absorção de uma material com densidade $N$ de sítios absorventes como

$$
\alpha(\hbar \omega)=\frac{N \hbar \omega W}{I_{0}}
$$

O coeficiente de absorção (4.45) depende da probabilidade $W$ de um fóton ser absorvido, a qual será calculada na seção seguinte considerando-se uma transição de dipolo elétrico, onde um elétron em um estado inicial $i$ absorve um fóton e é promovido para para um excitado final $f$, com uma energia $E_{f}=E_{i}+\hbar \omega$.

\subsubsection{Probabilidade de transição de um elétron}

A equação de Schrödinger para para o um elétron em um átomo descrito por um hamiltoniano $\mathcal{H}_{0}$ é dada por

$$
\mathcal{H}_{0} \Psi_{n}=\varepsilon_{n} \Psi_{n}
$$

onde $\varepsilon_{n}$ são os autovalores de $\mathcal{H}_{0}$ os quais denotam energia do $n$-ésimo estado eletrônico.

Um elétron no estado fundamental de energia $\varepsilon_{0}$, ao ser bombardeado por um feixe de luz, interage com o campo elétrico associado à onda eletromagnética e pode absorver um fóton de energia $\hbar \omega$, saltando para um estado de energia $\varepsilon_{0}+\hbar \omega$. Para descrever formalmente esse processo, vamos utilizar a teoria de perturbações dependente do tempo [75], onde o hamiltoniano do estado fundamental será resolvido e o efeito da luz será introduzido como uma correção na energia do estado fundamental. A correção será obtida a partir do potencial perturbativo devido à interação com o fóton, o qual será determinado a seguir. 
Uma partícula carregada de carga $q$, sob a ação de um campo elétrico $\boldsymbol{E}$, está sujeito à força de Lorentz, dada por

$$
\boldsymbol{F}=q \boldsymbol{E}
$$

A força de Lorentz é uma força conservativa e por isso é possível encontrar um potencial associado à mesma através da relação

$$
\boldsymbol{F}=-\nabla V
$$

de forma que, integrando (4.48) no espaço $\boldsymbol{r}$ percorrido pela carga obtemos

$$
\int \boldsymbol{F} d \boldsymbol{r}=-V
$$

Substituindo a força elétrica (4.47) em (4.49) obtemos

$$
V=-q \int \boldsymbol{E} d \boldsymbol{r}=-q \boldsymbol{E} \cdot \boldsymbol{r}
$$

Definindo-se o momento de dipolo

$$
\boldsymbol{\mu}=q \boldsymbol{r}
$$

o potencial da força elétrica da equação (4.50) pode ser escrito como

$$
V=-\mu \cdot \boldsymbol{E}
$$

O vetor campo elétrico em (4.52) é determinado pela equação de estado da onda eletromagnética, deduzida para as diversas polarizações na seção 4.1.2. Para um fóton de energia $\hbar \omega$ que se propaga na direção $z$ com polarização determinada pelo vetor de polarização $\hat{\xi}$, o vetor campo elétrico, no vácuo, é dado por

$$
\boldsymbol{E}(z, t)=\operatorname{Re}\left[\hat{\boldsymbol{\xi}} E_{0} e^{\imath(k z-\omega t)}\right]
$$

Esse campo elétrico desloca-se no eixo $z$, com um número de onda real $k=\frac{2 \pi}{\lambda}$. O produto $k z$ em (4.53) é dado por $\frac{2 \pi}{\lambda} \Delta z$, onde $\Delta z$ é a distância que o campo elétrico percorre nas imediações da interação. Se supusermos que o átomo que será excitado 
esteja contido num sólido cristalino de parâmetro de rede $a$, a interação ocorrerá em um sítio do cristal, que tem um tamanho característico $a$, portanto $\Delta z$ é da ordem de $a$, que usualmente é de alguns Ângstrons. Uma comparação entre as ordens de grandeza de $\Delta z \sim 10^{-10} m$ e do comprimento de onda do fóton de excitação $\left(\lambda \sim 10^{-7} m\right.$ no visível $)$ mostra que é possível aproximarmos $k \Delta z \simeq 0$, simplificando o potencial perturbativo para

$$
V(t)=-\operatorname{Re}\left[\hat{\xi} E_{0} e^{-\imath \omega t}\right] \cdot \boldsymbol{\mu}
$$

A equação de Schrödinger para um hamiltoniano que inclui esse potencial é

$$
\imath \hbar \frac{\partial \Psi(t)}{\partial t}=\left[\mathcal{H}_{0}+V(t)\right] \Psi(t)
$$

Na notação de Dirac, a mesma equação pode ser escrita como

$$
\imath \hbar \frac{\partial}{\partial t}|\Psi(t)\rangle=\left[\mathcal{H}_{0}+V(t)\right]|\Psi(t)\rangle
$$

Para resolver a equação (4.56) pode-se fazer uma expansão das soluções em autoestados do hamiltoniano não perturbado

$$
|\Psi(t)\rangle=\sum_{n} c_{n}(t) e^{-\frac{l}{\hbar} \varepsilon_{n} t}\left|\psi_{n}\right\rangle
$$

o que substituído em (4.56), após a diferenciação em relação ao tempo, resulta em

$$
\sum_{n}\left[\imath \hbar \frac{d}{d t} c_{n}(t)-V(t) c_{n}(t)\right] e^{-\frac{1}{\hbar} \varepsilon_{n} t}\left|\psi_{n}\right\rangle=0
$$

Tomando-se o produto interno de (4.58) com $\left\langle\psi_{m}\right|$, dividindo-se por $e^{-\frac{l}{\hbar} \varepsilon_{m} t}$ e utilizando a ortonormalidade dos autoestados não perturbados $\left\langle\psi_{m} \mid \psi_{n}\right\rangle=\delta_{m n}$ obtemos o conjunto de equações

$$
\imath \hbar \frac{d c_{m}(t)}{d t}=\sum_{n} c_{n}(t) e^{\frac{l}{\hbar}\left(\varepsilon_{m}-\varepsilon_{n}\right) t}\left\langle\psi_{m}|V(t)| \psi_{n}\right\rangle=\sum_{n} c_{n}(t) e^{\imath \omega_{m n} t} V_{m n}
$$

onde

$$
V_{m n}=\left\langle\psi_{m}|V(t)| \psi_{n}\right\rangle
$$


e $\omega_{m n} \equiv \frac{\varepsilon_{m}-\varepsilon_{n}}{\hbar}$ é a frequência de Bohr do sistema.

A probabilidade em um tempo $t$ de encontrarmos o sistema em um certo estado $k$ é dada por

$$
P_{k}(t)=\left|\left\langle\psi_{k} \mid \Psi(t)\right\rangle\right|^{2}=\left|c_{k}(t)\right|^{2}
$$

Se em $t=0$ o sistema encontra-se no estado $n$, deve-se observar $\left|c_{p}(0)\right|^{2}=\delta_{p n}$. Dessa maneira pode-se reescrever (4.59) para o estado $\left|\psi_{p}\right\rangle$ como

$$
\imath \hbar \frac{d c_{m}(t)}{d t}=\sum_{n \neq p}\left[c_{p}(0)+c_{n}(0)\right] V_{m n} e^{\imath \omega_{m n} t}=V_{m p} e^{\imath \omega_{m p} t}
$$

Para integrar (4.62) em relação ao tempo e obter a probabilidade de encontrar o sistema num estado $m$ em qualquer instante, deve-se notar que $V_{m p}$ depende do tempo e é dado por (4.60), que na notação integral pode ser escrito como

$$
V_{m p}=\int \psi_{m}^{*} V(t) \psi_{p} d \tau
$$

onde $d \tau$ significa que a integração é efetuada sobre todo o espaço. Substituindo o potencial (4.54) em (4.63) obtemos

$$
V_{m p}=-\int \psi_{m}^{*} \operatorname{Re}\left[\hat{\boldsymbol{\xi}} E_{0} e^{-\imath \omega t}\right] \cdot \boldsymbol{\mu} \psi_{p} d \tau=-\operatorname{Re}\left[\hat{\boldsymbol{\xi}} E_{0} e^{-\imath \omega t}\right] \cdot \int \psi_{m}^{*} \boldsymbol{\mu} \psi_{p} d \tau
$$

A integral

$$
\boldsymbol{\mu}_{m p}=-\int \psi_{m}^{*} \boldsymbol{\mu} \psi_{p} d \tau
$$

é denominada momento de transição. Com essa redução podemos escrever $V_{m p}$ na forma

$$
V_{m p}=\operatorname{Re}\left[\hat{\boldsymbol{\xi}} E_{0} e^{-\imath \omega t}\right] \cdot \boldsymbol{\mu}_{m p}
$$

Substituindo (4.66) em (4.62) obtemos

$$
\imath \hbar \frac{d c_{m}(t)}{d t}=\operatorname{Re}\left[E_{0} e^{-\imath \omega t} \hat{\boldsymbol{\xi}}\right] \cdot \boldsymbol{\mu}_{m p} e^{\imath \omega_{m p} t}
$$

Efetuando a operação $\operatorname{Re}[z]=\frac{z+z^{*}}{2}$, obtemos

$$
\imath \hbar \frac{d c_{m}(t)}{d t}=\frac{E_{0}}{2} e^{-\imath\left(\omega-\omega_{m p}\right) t} \hat{\boldsymbol{\xi}} \cdot \boldsymbol{\mu}_{m p}+\frac{E_{0}}{2} e^{\imath\left(\omega+\omega_{m p}\right) t} \hat{\boldsymbol{\xi}}^{*} \cdot \boldsymbol{\mu}_{m p}
$$


A integração em relação ao tempo de $t=0$ e $t=t$ resulta em

$$
c_{m}(t)=\frac{E_{0}}{2 \hbar}\left[\frac{e^{-\imath\left(\omega-\omega_{m p}\right) t}-1}{\left(\omega-\omega_{m p}\right)} \hat{\boldsymbol{\xi}}+\frac{e^{\imath\left(\omega+\omega_{m p}\right) t}-1}{\left(\omega+\omega_{m p}\right)} \hat{\boldsymbol{\xi}}^{*}\right] \cdot \boldsymbol{\mu}_{m p}
$$

Os dois termos entre colchetes tem significados físicos diferentes. Enquanto que o primeiro termo corresponde à absorção óptica, o segundo termo corresponda à emissão estimulada. O termo de absorção apresenta uma ressonância quando $\omega \simeq \omega_{m p}$, de forma que na condição de ressonância, esse termo será muito maior que o termo de emissão, e por isso esse último será descartado, de forma que a equação (4.69) é reduzida para

$$
c_{m}(t)=\frac{E_{0}}{2 \hbar} \frac{e^{-\imath\left(\omega-\omega_{m p}\right) t}-1}{\left(\omega-\omega_{m p}\right)} \hat{\boldsymbol{\xi}} \cdot \boldsymbol{\mu}_{m p}
$$

A probabilidade (4.61) de encontrar-se o sistema em um estado $m$ num instante de tempo $t$ pode ser obtida de (4.70),

$$
\begin{gathered}
\left|c_{m}(t)\right|^{2}=\left|\frac{E_{0} \hat{\boldsymbol{\xi}} \cdot \boldsymbol{\mu}_{m p}}{2 \hbar} \frac{e^{-l\left(\omega-\omega_{m p}\right) t}-1}{\left(\omega-\omega_{m p}\right)}\right|^{2}=\frac{E_{0}^{2}\left|\hat{\boldsymbol{\xi}} \cdot \boldsymbol{\mu}_{m p}\right|^{2}}{4 \hbar^{2}} \mid \frac{e^{l \frac{\omega_{m p}-\omega}{2} t}}{-\left(\omega_{m p}-\omega\right)} \underbrace{\left.\left(e^{i \frac{\omega_{m p}-\omega}{2} t}-e^{-l \frac{\omega_{m p}-\omega}{2} t}\right)\right|^{2}}_{2 \imath \operatorname{sen}\left(\frac{\omega_{m p}-\omega}{2} t\right)} \\
\therefore\left|c_{m}(t)\right|^{2}=\frac{E_{0}^{2}\left|\hat{\boldsymbol{\xi}} \cdot \boldsymbol{\mu}_{m p}\right|^{2}}{4 \hbar^{2}} \frac{\operatorname{sen}^{2}\left(\frac{\omega_{m p}-\omega}{2} t\right)}{\left|\frac{\omega_{m p}-\omega}{2}\right|^{2}}
\end{gathered}
$$

A taxa de transição, $W$, definida como a variação temporal da probabilidade da transição, é obtida por diferenciação da equação (4.71) em relação a $t$ :

$$
W=\frac{d}{d t}\left|c_{m}(t)\right|^{2}=\frac{E_{0}^{2}\left|\hat{\boldsymbol{\xi}} \cdot \boldsymbol{\mu}_{m p}\right|^{2}}{2 \hbar^{2}} \frac{\operatorname{sen}\left[\left(\omega_{m p}-\omega\right) t\right]}{\omega_{m p}-\omega}
$$

Num intervalo de tempo de $1 \mathrm{~s}$ para uma transição ressonante em $\sim 2 \mathrm{eV}$ temos $t \gg \frac{2 \pi}{\omega_{m p}}$, o que permite tomarmos o limite para $t \rightarrow \infty$ na equação (4.72), onde tem validade a seguinte propriedade da função delta de Dirac [76]:

$$
\lim _{t \rightarrow \infty} \frac{\operatorname{sen}(n t)}{n}=\pi \delta(n)
$$

Dessa forma a função $\frac{\operatorname{sen}\left[\left(\omega-\omega_{m p}\right) t\right]}{\omega_{m p}-\omega}$ em $(4.72)$ pode ser aproximada por $\pi \delta\left(\omega_{m p}-\omega\right)$ e a taxa de transição $W$ fica

$$
W=\frac{\pi E_{0}^{2}\left|\hat{\boldsymbol{\xi}} \cdot \boldsymbol{\mu}_{m p}\right|^{2}}{2 \hbar^{2}} \delta\left(\omega_{m p}-\omega\right)
$$


Utilizando também a propriedade $\frac{\delta(\omega)}{|\hbar|}=\delta(\hbar \omega)$ da delta de Dirac, a equação (4.74) pode ser reescrita em termos das energias

$$
W=\frac{\pi E_{0}^{2}\left|\hat{\boldsymbol{\xi}} \cdot \boldsymbol{\mu}_{m p}\right|^{2}}{2 \hbar} \delta\left(E_{m p}-\hbar \omega\right)
$$

onde $E_{m p}=E_{m}-E_{p}$ é a diferença de energia entre os estados inicial e final. A forma geral

$$
\Gamma_{i \rightarrow f}=\frac{2 \pi}{\hbar}\langle i|\gamma| f\rangle \delta\left(E_{f}-E_{i}\right)
$$

da taxa de transição de um estado $i$ de energia $E_{i}$ para um estado $f$ de energia $E_{f}$ através duma perturbação $\gamma$ tem validade em diversos sistemas físicos e por isso foi batizada por E. Fermi como "Regra de Ouro n`2" [77].

\subsubsection{Transição óptica num sistema de muitos elétrons}

A solução da equação de Schrödinger por teoria de perturbação em primeira ordem (4.75) forneceu a taxa de transição $W$ de um elétron. Para um sistema com $n$ elétrons, o momento da transição $\boldsymbol{\mu}_{m p}=-\langle m|\boldsymbol{\mu}| p\rangle$ (cf. equação (4.65)) deve ser contabilizado entre estados caracterizados por funções de onda de $n$ elétrons. O operador dipolo elétrico $\boldsymbol{\mu}=q \boldsymbol{r}$ deve poder atuar em qualquer um dos $n$ elétrons, passando portanto, a ser uma soma dos $n$ operadores de um elétron

$$
\begin{aligned}
\boldsymbol{\mu}=q \boldsymbol{r} & \rightarrow \sum_{j=1}^{n} q \boldsymbol{r}_{j} \\
\varphi_{m}(\boldsymbol{r}) & \rightarrow \varphi_{m}\left(\boldsymbol{r}_{1}, \ldots, \boldsymbol{r}_{n}\right) \\
\varphi_{p}(\boldsymbol{r}) & \rightarrow \varphi_{p}\left(\boldsymbol{r}_{1}, \ldots, \boldsymbol{r}_{n}\right)
\end{aligned}
$$

Fazendo as substituições acima em (4.65) e substituindo $q$ pela carga do elétron $-e$, obtemos o momento de transição de um elétron em um estado inicial $i$ para um estado final $f$

$$
\boldsymbol{\mu}_{f i}=\left\langle\varphi_{f}\left(\boldsymbol{r}_{1}, \ldots, \boldsymbol{r}_{n}\right)\left|\sum_{j=1}^{n} e \boldsymbol{r}_{j}\right| \varphi_{i}\left(\boldsymbol{r}_{1}, \ldots, \boldsymbol{r}_{n}\right)\right\rangle
$$


Introduzindo o momento de transição multieletrônico em (4.75), obtemos a taxa de transição de um sistema de $n$ elétrons como

$$
W_{f i}=\frac{\pi E_{0}^{2} e^{2}}{2 \hbar}\left|\left\langle\varphi_{f}\left(\boldsymbol{r}_{1}, \ldots, \boldsymbol{r}_{n}\right) \hat{\boldsymbol{\xi}} \cdot \sum_{j=1}^{n} \boldsymbol{r}_{j} \mid \varphi_{i}\left(\boldsymbol{r}_{1}, \ldots, \boldsymbol{r}_{n}\right)\right\rangle\right|^{2} \delta\left(E_{f i}-\hbar \omega\right)
$$

onde $E_{f i}$ é a diferença de energia entre os estados final e inicial, que deve corresponder à energia do fóton incidente para que o balanço energético seja satisfeito e função delta não se anule.

Em um sistema com um número $Q$ de estados finais de energia $E_{f}$, o coeficiente de absorção deve ser somado para todas as transições entre o estado inicial e os possíveis estados finais. Substituindo o resultado da equação (4.35)

$$
I_{0}=\frac{1}{2} c \epsilon_{0} E_{0}^{2}
$$

e o da equação (4.81) na equação (4.45)

$$
\alpha(\hbar \omega)=\frac{N \hbar \omega W}{I_{0}}
$$

obtemos coeficiente de absorção de um átomo de $n$ elétrons como

$$
\alpha(\hbar \omega)=\sum_{f}^{Q} \frac{\pi N e^{2} \hbar \omega}{c \epsilon_{0} \hbar}\left|\left\langle\varphi_{f}\left(\boldsymbol{r}_{1}, \ldots, \boldsymbol{r}_{n}\right)\left|\hat{\boldsymbol{\xi}} \cdot \sum_{j=1}^{n} \boldsymbol{r}_{j}\right| \varphi_{i}\left(\boldsymbol{r}_{1}, \ldots, \boldsymbol{r}_{n}\right)\right\rangle\right|^{2} \delta\left(E_{f}-E_{i}-\hbar \omega\right)
$$

Esse resultado será implementado nos EuX, substituindo as funções de onda gerais em (4.84) pelas funções de onda que caracterizam os estados eletrônicos dos EuX, o que será o assunto da próxima seção.

\subsubsection{Níveis de energia dos EuX}

A distribuição eletrônica dos íons $\mathrm{Eu}^{2+}$ no estado fundamental resulta em camadas completas equivalentes à distribuição de um átomo de xenônio neutro, mais sete elétrons de spins paralelos no orbital $4 f$, levando a um spin total $S=7 / 2$ e momento angular $\boldsymbol{L}=0$. Como visto na seção 2.3.1, esse estado pode ser representado na notação espectroscópica por um termo ${ }^{8} S_{7 / 2}$ então vamos representar a função de onda do 
estado fundamental como

$$
\left|\varphi_{i}\left(\boldsymbol{r}_{1}, \ldots, \boldsymbol{r}_{n}\right)\right\rangle=\left|{ }^{8} S_{\frac{7}{2}}\right\rangle
$$

A energia desse estado é dada pelo autovalor do hamiltoniano do sistema $\mathcal{H}$,

$$
E=\left\langle{ }^{8} S_{\frac{7}{2}}|\mathcal{H}|^{8} S_{\frac{7}{2}}\right\rangle
$$

entretanto para o cálculo do coeficiente de absorção não é necessário calcular explicitamente $E_{4 f}$, uma vez que estamos interessados na diferença de energia entre os estados fundamental e excitado, por isso vamos definir a energia do estado fundamental como a origem da escala de energias.

No estado excitado de menor energia, um dos elétrons do íon $\mathrm{Eu}^{2+}$ é transferido para o fundo da banda de condução, que é composta por orbitais atômicos $5 d\left(t_{2 g}\right)$ e que será examinada em detalhe na seção 4.1.6. Os seis demais elétrons permanecem no orbital $4 f$ e na configuração de menor energia, de acordo com a primeira e segunda regra de Hund tem seus momentos angulares orbital e de spin maximizados, o que implica em $\boldsymbol{L}=3$ e $\boldsymbol{S}=3$. Isso implica que quando um elétron deixa o orbital $4 f$, surge uma interação spin-órbita que acopla $\boldsymbol{L}$ e $\boldsymbol{S}$ no momento angular total $\boldsymbol{J}=\boldsymbol{L}+\boldsymbol{S}$, ocasionando um desdobramento nos níveis de energia desse orbital. O estado $4 f^{6}$ na notação espectroscópica corresponde a um termo ${ }^{7} F_{J}$ e, inserindo também a projeção $M$ de $J$ no eixo $z$, rotularemos a função de onda desse estado por

$$
\left|4 f^{6}\right\rangle=\left|{ }^{7} F_{J M}\right\rangle
$$

No formalismo da teoria de perturbação até de primeira ordem, o desdobramento de energia pode ser obtido pelo valor médio do hamiltoniano de spin-órbita nos autoestados não perturbados. Esse hamiltoniano é dado por

$$
\mathcal{H}_{S O}=\frac{\lambda_{4 f}}{\hbar^{2}} \boldsymbol{L} \cdot \boldsymbol{S}
$$

onde $\lambda_{4 f}$ é uma constante que caracteriza a interação spin-órbita. Para efetuar o produto interno, observamos que $\boldsymbol{J}=\boldsymbol{L}+\boldsymbol{S}$. Elevando-se ambos os lados à segunda potência e rearranjando os termos obtemos

$$
\boldsymbol{L} \cdot \boldsymbol{S}=\frac{1}{2}\left(J^{2}-L^{2}-S^{2}\right)
$$


o que substituído na equação (4.88) permite escrever o hamiltoniano de spin-órbita em termos de operadores que comutam com o hamiltoniano não perturbado

$$
\mathcal{H}_{\mathrm{SO}}=\frac{\lambda_{4 f}}{2 \hbar^{2}}\left(J^{2}-L^{2}-S^{2}\right)
$$

de forma que a energia desses estados pode ser calculada por

$$
\begin{array}{r}
\left\langle{ }^{7} F_{J M}\left|\mathcal{H}_{\mathrm{SO}}\right|{ }^{7} F_{J M}\right\rangle=\left\langle{ }^{7} F_{J M}\left|\frac{\lambda_{4 f}}{2 \hbar^{2}}\left(J^{2}-L^{2}-S^{2}\right)\right|{ }^{7} F_{J M}\right\rangle= \\
=\lambda_{4 f} \frac{J(J+1)-3(3+1)-3(3+1)}{2}\left\langle{ }^{7} F_{J M} \mid{ }^{7} F_{J M}\right\rangle=\lambda_{4 f} \frac{J(J+1)-24}{2}
\end{array}
$$

Pela terceira regra de Hund, o estado de mínima energia é aquele para o menor valor do momento angular total $\boldsymbol{J}$, uma vez que a camada $4 f$ encontra-se com menos do que a metade de seus níveis populados, portanto o nível de menor energia é aquele correspondente a $\boldsymbol{J}=0$. Como definimos a energia zero como a do estado fundamental, a menor energia para o estado $4 f^{6}$ é a correspondente à energia do gap, $E_{G}$, de forma que, das equações (4.91) obtemos

$$
E_{J}=E_{G}+\lambda_{4 f} \frac{J(J+1)}{2}
$$

Cada valor de $J=0,1, \ldots, 6$ corresponde a um possível estado eletrônico de energia $E_{J}$, o qual é separado do próximo estado $J+1$ por uma energia $\Delta E_{J}$,

$$
\Delta E_{J}=E_{J+1}-E_{J}=\lambda_{4 f}(J+1)
$$

o que é conhecido como a regra do intervalo de Landé. Um resumo dos níveis de energia envolvidos na transição está apresentado na figura 4.4 .

\section{Função de onda do elétron fotoexcitado}

Como mencionado na seção anterior, a absorção de um fóton por um elétron de um íon $\mathrm{Eu}^{2+}$ promove um esse elétron para uma banda de condução que será construída a partir de funções de onda atômicas $5 d$. Os orbitais $d$ são caracterizados pelo número quântico $\ell=2$, que pode ter projeções no eixo $z, m_{\ell}=-2,-1,0,1,2$. Dependendo dos valores de $m_{\ell}$, diferentes distribuições de carga são encontradas nesses orbitais, são elas $d_{x y}, d_{x z}$, 


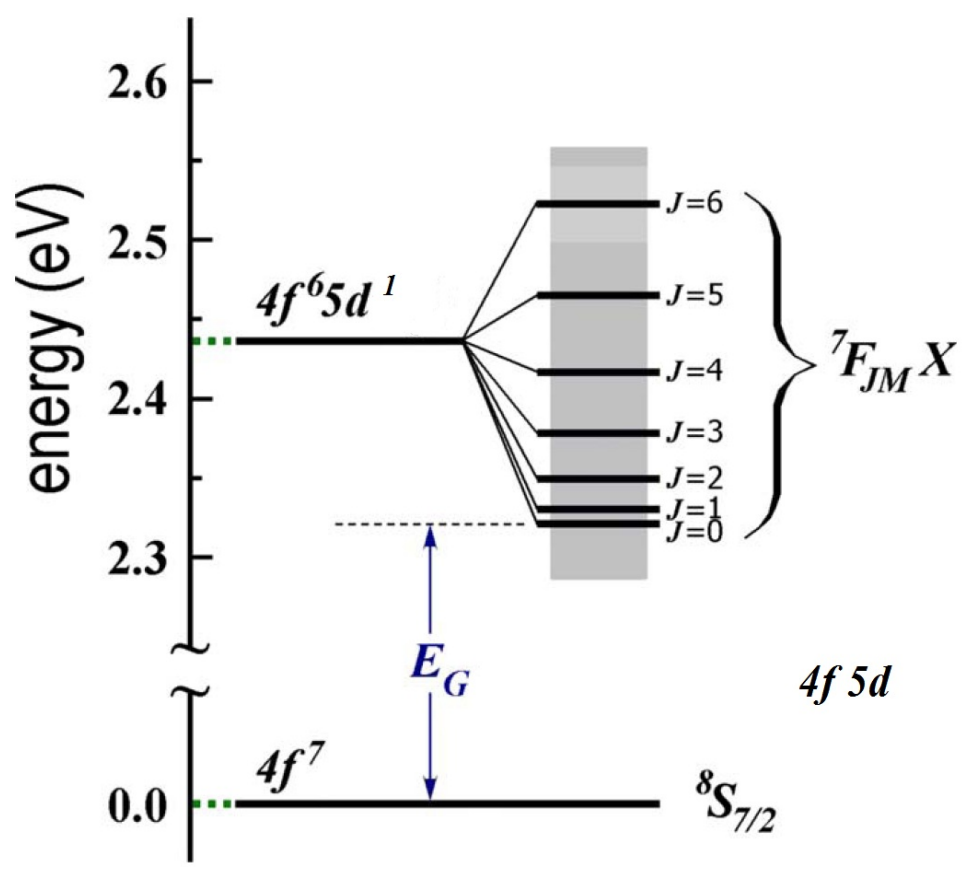

Figura 4.4: Diagrama de níveis de energia para os EuX no modelo da transição óptica $4 f \rightarrow 5 d$

$d_{y z}, d_{z^{2}}$ e $d_{x^{2}-y^{2}}$, ilustradas na figura 4.5. Num átomo isolado, essas cinco distribuições são degeneradas em energia, entretanto, em um sólido o campo cristalino dos átomos vizinhos quebra parcialmente essa degenerescência. Na estrutura $f c c$ dos EuX, cada íon $\mathrm{Eu}^{2+}$ encontra-se no centro de um octaedro de íons $\mathrm{X}^{2-}$, representados pelas esferas verdes na figura 4.5. Os átomos ligantes $\mathrm{X}^{2-}$, estão mais próximos dos elétrons $d$ do európio para as distribuições $d_{z^{2}}$ e $d_{x^{2}-y^{2}}\left(e_{g}\right)$, do que para as distribuições $d_{x y}, d_{x z}$ e $d_{y z}$ $\left(t_{2 g}\right)$, o que torna a energia eletrostática maior para os níveis $5 d\left(e_{g}\right)$ comparada à energia dos níveis $5 d\left(t_{2 g}\right)$.

Os orbitais $d$ são obtidos por combinações lineares de funções de onda atômicas $\phi_{5,2, m}$, que de forma geral podemos representar por uma função de onda

$$
\phi(\boldsymbol{r}-\boldsymbol{R})=\sum_{m=-2}^{2} B(X, m) \Psi_{5,2, m}(\boldsymbol{r}-\boldsymbol{R})|\sigma\rangle
$$

onde $X$ representa um dos cinco possíveis orbitais $d, B(X, m)$ são os coeficientes da combinação linear normalizada, que serão determinados a seguir e $|\sigma\rangle$ representa a parte de spin da função de onda, que depende do número quântico $m_{s}= \pm 1 / 2$. 

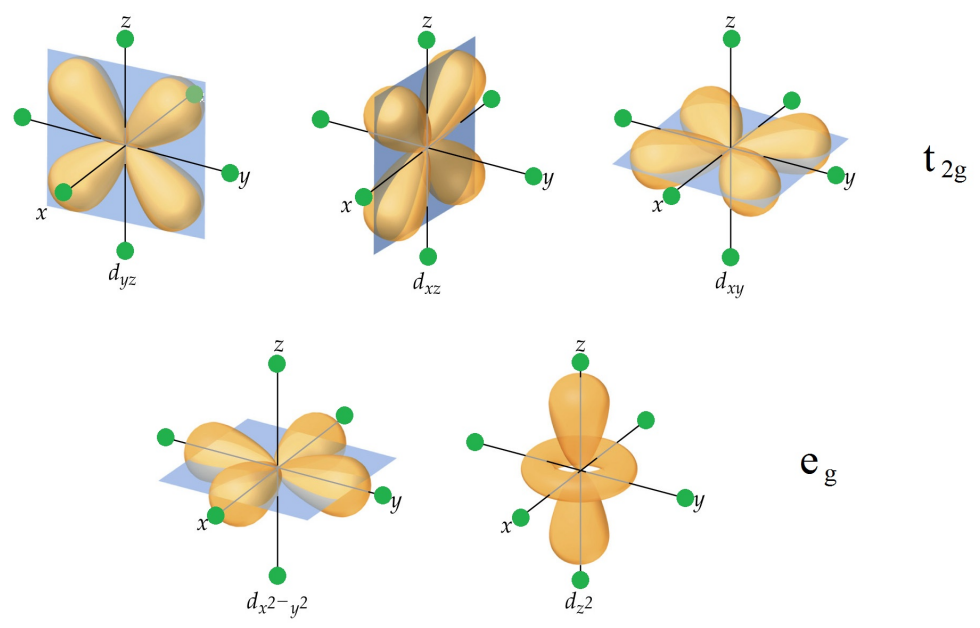

$\mathrm{e}_{\mathrm{g}}$

Figura 4.5: Em amarelo vemos a distribuição de carga dos orbitais $d$. As esferas verdes representam os átomos ligantes, que introduzem a separação dos orbitais $d$ nos níveis $d\left(t_{2_{g}}\right)$ e $d\left(e_{g}\right)$.

Para determinar os coeficientes $B(X, m)$, notamos que a parte espacial das funções de onda atômicas, $\psi_{5,2, m}$, é separável em suas componentes radial e angular. A parte radial não depende de $m$ e será denotada por $R_{5,2}(r)$. A parte angular é dada pelos harmônicos esféricos $Y_{2, m}$, cujas formas explícitas podem ser encontradas, por exemplo, na referência [78]. Em coordenadas esféricas, as funções de onda $\psi_{5,2, m}$ são dadas por

$$
\begin{aligned}
\psi_{5,2,0} & =R_{5,2}(r) \sqrt{\frac{5}{16 \pi}}\left(1-3 \cos ^{2} \theta\right) \\
\psi_{5,2, \pm 1} & = \pm R_{5,2}(r) \sqrt{\frac{15}{8 \pi}} \cos \theta \operatorname{sen} \theta e^{ \pm l \phi} \\
\psi_{5,2, \pm 2} & =-R_{5,2}(r) \sqrt{\frac{15}{32 \pi}} \operatorname{sen}^{2} \theta e^{ \pm 2 \imath \phi}
\end{aligned}
$$

Em coordenadas cartesianas as mesmas funções de onda são escritas como

$$
\begin{aligned}
\psi_{5,2,0} & =R_{5,2}(r) \sqrt{\frac{5}{16 \pi}}\left(1-3\left(\frac{z}{r}\right)^{2}\right) \\
\psi_{5,2, \pm 1} & = \pm R_{5,2}(r) \sqrt{\frac{15}{8 \pi}} \frac{1}{r^{2}}(x z \pm \imath y z) \\
\psi_{5,2, \pm 2} & =-R_{5,2}(r) \sqrt{\frac{15}{32 \pi}} \frac{1}{r^{2}}\left[\left(x^{2}-y^{2}\right) \pm \imath x y\right]
\end{aligned}
$$


Definindo-se $\frac{R_{5,2}(r)}{r^{2}}=g(r) \mathrm{e}$

$$
\begin{aligned}
d_{x y} & =g(r) \sqrt{\frac{15}{32 \pi}} x y \\
d_{x z} & =g(r) \sqrt{\frac{15}{8 \pi}} x z \\
d_{y z} & =g(r) \sqrt{\frac{15}{8 \pi}} y z \\
d_{x^{2}-y^{2}} & =g(r) \sqrt{\frac{15}{32 \pi}}\left(x^{2}-y^{2}\right) \\
d_{z^{2}} & =\sqrt{\frac{5}{16 \pi}} g(r)\left(r^{2}-3 z^{2}\right)
\end{aligned}
$$

podemos reescrever as equações (4.96) como

$$
\begin{aligned}
\psi_{5,2,0} & =d_{z^{2}} \\
\psi_{5,2,+1} & =d_{x z}+\imath d_{y z} \\
\psi_{5,2,-1} & =-d_{x z}+\imath d_{y z} \\
\psi_{5,2,+2} & =-d_{x^{2}-y^{2}}-\imath d_{x y} \\
\psi_{5,2,-2} & =-d_{x^{2}-y^{2}}+\imath d_{x y}
\end{aligned}
$$

de onde podemos identificar

$$
\begin{aligned}
d_{z^{2}} & =\psi_{5,2,0} \\
d_{x z} & =\frac{1}{2}\left[\psi_{5,2,+1}-\psi_{5,2,-1}\right] \\
d_{y z} & =\frac{1}{2 \imath}\left[\psi_{5,2,+1}+\psi_{5,2,-1}\right] \\
d_{x^{2}-y^{2}} & =-\frac{1}{2}\left[\psi_{5,2,+2}+\psi_{5,2,-2}\right] \\
d_{x y} & =-\frac{1}{2 \imath}\left[\psi_{5,2,+2}-\psi_{5,2,-2}\right]
\end{aligned}
$$

Para que essas funções de onda sejam normalizadas, deve-se calcular os fatores de normalização $\sqrt{N}$, determinados por $N \int \Psi_{X}^{*} \Psi_{X} d \tau=1$, para cada uma delas. Sabendo que $\int \psi_{5,2, m}^{*} \psi_{5,2, n} d \tau=\delta_{m, n}$, encontramos $N$ para cada função de onda, de forma que 
multiplicando as equações (4.99) por $\sqrt{N}$ obtemos

$$
\begin{aligned}
d_{z^{2}} & =\psi_{5,2,0} \\
d_{x z} & =\frac{\sqrt{2}}{2}\left[\psi_{5,2,+1}-\psi_{5,2,-1}\right] \\
d_{y z} & =\frac{\sqrt{2}}{2 \imath}\left[\psi_{5,2,+1}+\psi_{5,2,-1}\right] \\
d_{x^{2}-y^{2}} & =-\frac{\sqrt{2}}{2}\left[\psi_{5,2,+2}+\psi_{5,2,-2}\right] \\
d_{x y} & =-\frac{\sqrt{2}}{2 \imath}\left[\psi_{5,2,+2}-\psi_{5,2,-2}\right]
\end{aligned}
$$

de onde podemos determinar os coeficientes $B(X, m)$, que estão apresentados na tabela 4.1 .

\begin{tabular}{c|ccccc}
\hline & \multicolumn{5}{c}{ Tabela 4.1: Coeficientes $B(X, m)$} \\
\hline & 2 & 1 & 0 & -1 & -2 \\
$d_{x z}$ & 0 & $\frac{\sqrt{2}}{2}$ & 0 & $-\frac{\sqrt{2}}{2}$ & 0 \\
$d_{y z}$ & 0 & $\frac{\sqrt{2}}{2 \imath}$ & 0 & $\frac{\sqrt{2}}{2 \imath}$ & 0 \\
$d_{x y}$ & $-\frac{\sqrt{2}}{2 \imath}$ & 0 & 0 & 0 & $\frac{\sqrt{2}}{2 \imath}$ \\
$d_{x^{2}-y^{2}}$ & $-\frac{\sqrt{2}}{2}$ & 0 & 0 & 0 & $-\frac{\sqrt{2}}{2}$ \\
$d_{z^{2}}$ & 0 & 0 & 1 & 0 & 0
\end{tabular}

A separação entre os níveis $5 d\left(e_{g}\right)$ e $5 d\left(t_{2 g}\right)$ é de cerca de $1 \mathrm{eV}$ para os EuX [19], portanto o limiar da absorção é dado pelas transições que ocorrem entre o nível $4 f$ e a banda $5 d\left(t_{2 g}\right)$, de forma que o estado $X$ será um estado de Bloch construído a partir de orbitais $5 d\left(t_{2 g}\right)$.

A equação de Schrödinger do elétron num potencial $V(\boldsymbol{r})$ é

$$
\left(-\frac{\hbar^{2}}{2 m_{e}}+V(\boldsymbol{r})\right) \Psi(\boldsymbol{r})=E \Psi(\boldsymbol{r})
$$


No modelo do campo efetivo, o efeito de todos os íons do cristal sobre o estado $|X\rangle$ é agrupado em um potencial cristalino, que é idêntico em cada célula primitiva do cristal e portanto possui a mesma periodicidade da rede. Para um elétron sob a ação de um potencial periódico, tem validade o Teorema de Bloch, o qual estabelece que os autoestados do hamiltoniano desse elétron sempre podem ser representados como o produto de uma onda plana com uma função periódica, que tem a mesma periodicidade da rede,

$$
\Psi_{n k}(\boldsymbol{r})=e^{i k \cdot r} u_{n k}(r)
$$

onde $\boldsymbol{k}$ é um vetor da rede recíproca e $u_{n k}(\boldsymbol{r}+\boldsymbol{R})=u_{n k}(\boldsymbol{r})$, para qualquer $\boldsymbol{R}$ da rede.

Os autoestados de Bloch do hamiltoniano são periódicos no espaço recíproco, de forma que $\Psi(\boldsymbol{k}+\boldsymbol{K}, \boldsymbol{r})=\Psi(\boldsymbol{k}, \boldsymbol{r})$, onde $\boldsymbol{K}$ é qualquer vetor da rede recíproca, o que implica que esses estados podem ser expandidos numa série de Fourier

$$
\Psi_{m}(\boldsymbol{k}, \boldsymbol{r})=\frac{1}{\sqrt{\mathcal{N}}} \sum_{n} w_{m}\left(\boldsymbol{R}_{n}, \boldsymbol{r}\right) e^{i \boldsymbol{k} \cdot \boldsymbol{R}_{n}}
$$

onde $\mathcal{N}$ é o número de sítios no volume de Born-von Karmán e as funções $w_{m}\left(\boldsymbol{R}_{n}, \boldsymbol{r}\right)$ são chamadas funções de Wannier, as quais são bem-localizadas em sítios individuais da rede.

Como a distância entre dois sítios adjacentes é muito grande nos EuX, o recobrimento entre as funções de Wannier é desprezível, portanto é possível restringir a somatória em $\boldsymbol{R}$ ao sítio onde ocorreu a excitação $(\boldsymbol{R}=0)$ e aproximar as funções de Wannier pelas funções de onda atômicas $5 d\left(t_{2 g}\right)$ da equação (4.94), as quais substituídas em (4.103) resultam na seguinte função para o elétron fotoexcitado

$$
|X(\boldsymbol{r})\rangle=\frac{1}{\sqrt{\mathcal{N}}} \sum_{m=-2}^{2} B(X, m) \Psi_{5,2, m}(\boldsymbol{r})|\sigma\rangle
$$

O elétron no estado $|X\rangle$ está num estado de baixa localidade e interage por exchange com os sítios da rede, portanto a energia do estado $|X\rangle, E_{X}$, terá além da componente dependente de $\boldsymbol{k}, E(\boldsymbol{k})$, uma componente associada à interação do elétron com os spins da rede. 
Na seção 2.1.2 foi estabelecido que a interação de exchange pode ser formulada em termos de um hamiltoniano efetivo, o hamiltoniano de Heisenberg. Para formular o hamiltoniano de Heisenberg da interação de exchange $d-f$, vamos considerar que o spin do elétron de condução é descrito por um operador $s_{d}$. A função de onda do elétron percorre todos os sítios da rede, portanto o elétron fotoexcitado interage com os $i$ íons $\mathrm{Eu}^{2+}$, situados na posição $\boldsymbol{R}_{i}$, através de uma constante de interação $J\left(\boldsymbol{r}-\boldsymbol{R}_{i}\right)$, associada a cada sítio da rede. Portanto o hamiltoniano de Heisenberg (2.27) para essa interação pode ser escrito como

$$
\mathcal{H}_{X}=-2 \sum_{i} J\left(\boldsymbol{r}-\boldsymbol{R}_{i}\right) \boldsymbol{s}_{d} \cdot \boldsymbol{S}_{i}
$$

Se for feita a suposição de que a função de onda $|X\rangle$ é distribuída uniformemente nos $\mathcal{N}$ sítios com a qual possui recobrimento apreciável e que seja nula nos demais sítios, pode-se definir uma constante de exchange, $J_{d f}$, que caracteriza a interação do elétron fotoexcitado (num estado $d$ ) $\operatorname{com}$ os $\mathcal{N}$ sítios da rede recobertos, de forma que o hamiltoniano de exchange (4.105) pode ser exprimido como

$$
\mathcal{H}_{d f}=-2 \sum_{i}^{\mathcal{N}} J\left(\boldsymbol{r}-\boldsymbol{R}_{i}\right) \boldsymbol{s}_{d} \cdot \boldsymbol{S}_{i}=-\frac{2 J_{d f}}{\mathcal{N}} \sum_{i}^{\mathcal{N}} \boldsymbol{s}_{d} \cdot \boldsymbol{S}_{i}
$$

Esse hamiltoniano depende da orientação dos spins da rede, que é diferente para cada EuX. Nos casos do EuO e do EuS, o ordenamento é FM, de forma que $\boldsymbol{s}_{d} \cdot \boldsymbol{S}_{i}=1$ e a energia de exchange é igual em todos os sítios e dada pela constante $J_{d f} S$ para $T<T_{C}$. Nos casos do EuTe e do EuSe, em $T=0 \mathrm{~K}$, o ordenamento dos spins é AFMII. Para esse ordenamento de spins, a energia de exchange pode ser determinada como se segue.

\section{Exchange $d-f$ na fase AFMII}

No arranjo AFMII da rede $f\left(c\right.$, a rede pode ser dividida em duas sub-redes, $S_{1}$ e $S_{2}$, cada qual contendo $\mathcal{N} / 2$ átomos de Eu recobertos pela função de onda do elétron no estado $5 d$. A energia de exchange do elétron fotoexcitado com os spins da rede (4.106) nesse caso é

$$
\mathcal{H}_{d f}=-\frac{2 J_{d f}}{\mathcal{N}} \boldsymbol{s}_{d} \cdot\left(\frac{\mathcal{N}}{2} \boldsymbol{S}_{1}+\frac{\mathcal{N}}{2} \boldsymbol{S}_{2}\right)=-J_{d f} \boldsymbol{s}_{d} \cdot\left(\boldsymbol{S}_{1}+\boldsymbol{S}_{2}\right)
$$


Para efetuar os produtos escalares em (4.107), notemos que as sub-redes $S_{1}$ e $S_{2}$ são idênticas, exceto pela orientação de seus spins. Em campo nulo, os spins das sub-redes $S_{1}$ e $S_{2}$ apontam em sentidos opostos, de forma que

$$
s_{d} \cdot S_{1}=-s_{d} \cdot S_{2}
$$

e as contribuições de cada sub-rede em (4.107) são canceladas, indicando que a energia de exchange ganha com uma das sub-redes é perdida com a outra sub-rede.

Quando aplica-se um campo superior a $B_{s f}$, os spins da rede inclinam-se para a direção do campo formando um ângulo $\theta$ com essa direção, como está representado na figura 4.6.

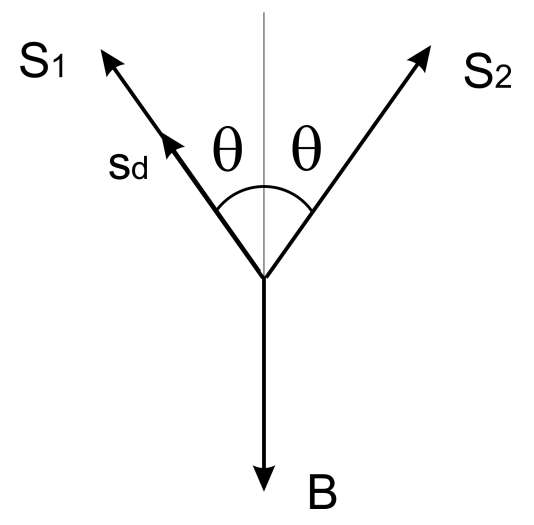

Figura 4.6: Spins das sub-redes na presença de um campo magnético $B$ e a conservação da direção do spin $s_{d}$ do elétron fotoexcitado.

O spin do elétron fotoexcitado tem a sua direção conservada na transição de dipolo elétrico, de modo que durante a absorção essa direção é a direção dos spins da sua sub-rede original (figura 4.6). Assim,

$$
\begin{array}{r}
s_{d} \cdot \boldsymbol{S}_{1}=\left|s_{d} \| \boldsymbol{S}_{1}\right| \cos 0=\frac{S}{2} \\
\boldsymbol{s}_{d} \cdot \boldsymbol{S}_{2}=\left|\boldsymbol{s}_{d}\right|\left|\boldsymbol{S}_{2}\right| \cos 2 \theta=\frac{S}{2} \cos 2 \theta
\end{array}
$$

de forma que a energia de exchange (4.107) fica dada por

$$
\mathcal{H}_{d f}=-J_{d f} \frac{S}{2}(1+\cos 2 \theta)=-J_{d f} S \cos ^{2} \theta
$$


Para obter o ângulo $\theta$ em função de $B$, vamos minimizar a energia total dos spins da rede em relação a $\theta$. Essa energia é dada pela interação de exchange dos spins da rede, pela interação Zeeman dos spins com o campo magnético e pelo potencial de anisotropia. Entretanto esse último termo pode ser descartado se supusermos um campo aplicado superior a $B_{s f}$. Como feito na seção 2.3.5, vamos considerar interações de exchange com primeiros e segundos vizinhos, caracterizadas pelas constantes $J_{1}$ e $J_{2}$. Cada sítio de Eu na rede $f c c$ possui 6 primeiros vizinhos na sub-rede $S_{1}$ e 6 primeiros vizinhos e 6 segundos vizinhos na sub-rede $S_{2}$, de forma que a energia de exchange do $n$-ésimo spin da rede com todos os primeiros e segundos vizinhos é dada por vizinhos é dada por

$$
E_{\mathrm{x}}=-6 J_{1} S^{2}-6 J_{1} S^{2} \cos 2 \theta-6 J_{2} S^{2} \cos 2 \theta
$$

A energia da interação Zeeman é dada por

$$
E_{Z}=-\boldsymbol{\mu} \cdot \boldsymbol{B}
$$

onde $\boldsymbol{\mu}=-g \mu_{B} \boldsymbol{S}$ é momento magnético de um íon $\mathrm{Eu}^{2+}$. Como $\boldsymbol{\mu}$ tem sentido contrário a $S$, o ângulo entre $\boldsymbol{\mu}$ e $\boldsymbol{B}$ é $(\pi-\theta)$, de forma que efetuando o produto escalar em (4.110) obtemos $E_{Z}=-g \mu_{B} S \cos \theta$. Com esse resultado a energia total $E_{\mathrm{x}}+E_{Z}$ de um spin da rede na presença do campo magnético é

$$
E=-6 J_{1} S^{2}-6 J_{1} S^{2} \cos 2 \theta-6 J_{2} S^{2} \cos 2 \theta-g \mu_{B} S B \cos \theta
$$

cuja minimização em relação a $\theta, \frac{\partial E}{\partial \theta}=0$ resulta em

$$
\cos \theta=\frac{g \mu_{B} B}{24\left|J_{1}+J_{2}\right| S}
$$

o que implica que

$$
\cos \theta= \begin{cases}\frac{B}{B_{S A T}}, & B<B_{S A T} \\ 1, & B \geq B_{S A T}\end{cases}
$$

onde definimos o campo de saturação

$$
B_{S A T}=\frac{24\left|J_{1}+J_{2}\right| S}{g \mu_{B}}
$$


que corresponde à menor magnitude de campo magnético para que os spins da rede encontrem-se totalmente alinhados à direção do campo.

Assim, o elétron fotoexcitado ganha energia progressivamente na medida que o campo magnético alinha os spins da rede para a direção do spin do elétron, até que o campo de saturação seja atingido, quando todos os spins estão alinhados e ganho de energia do elétron é máximo. A substituição de (4.112) na equação (4.108) permite relacionar a energia de exchange $d-f$ com a magnitude do campo aplicado,

$$
\Delta E= \begin{cases}-J_{d f} S\left(\frac{B}{B_{S A T}}\right)^{2}, & B<B_{S A T} \\ -J_{d f} S, & B \geq B_{S A T}\end{cases}
$$

revelando um ganho de energia quadrático em $B$ até o campo de saturação, e constante para campos maiores.

Assim, a energia do elétron fotoexcitado para o arranjo AFMII é dada por

$$
E_{X}=E(\boldsymbol{k})+\Delta E
$$

Para outros arranjos dos spins da rede, a energia de exchange pode ser calculada de maneira análoga.

Agora que foram estabelecidos os estados inicial e final da transição óptica e suas respectivas energias, é possível proceder com o cálculo do coeficiente de absorção, que é o tema da próxima seção.

\subsubsection{Cálculo do coeficiente de absorção nos EuX}

Na equação (4.84) foi mostrado que o coeficiente de absorção de um átomo de muitos elétrons é dado por

$$
\alpha(\hbar \omega)=\sum_{f}^{Q} \frac{\pi N e^{2} \hbar \omega}{c \epsilon_{0} \hbar}\left|\left\langle\varphi_{f}\left(\boldsymbol{r}_{1}, \ldots, \boldsymbol{r}_{n}\right)\left|\hat{\boldsymbol{\xi}} \cdot \sum_{j=1}^{n} \boldsymbol{r}_{j}\right| \varphi_{i}\left(\boldsymbol{r}_{1}, \ldots, \boldsymbol{r}_{n}\right)\right\rangle\right|^{2} \delta\left(E_{f}-E_{i}-\hbar \omega\right)
$$

O estado inicial corresponde aos elétrons no orbital $4 f$, cuja função de onda foi determinada na equação (4.85) como

$$
\varphi_{i}\left(\boldsymbol{r}_{1}, \ldots, \boldsymbol{r}_{n}\right)=\left|{ }^{8} S_{\frac{7}{2}}\right\rangle
$$


e cuja energia energia $E_{i}$ foi definida como o nível zero de energia.

O estado final é composto de duas partes: seis elétrons que permanecem na camada $4 f$, os quais formam um orbital $\left|{ }^{7} F_{J M}\right\rangle$, de energia $E_{J}=\frac{\lambda_{4 f}}{2} J(J+1)$, onde $J=0,1, \ldots, 6$, mais o elétron que ocupa um orbital $5 d\left(t_{2 g}\right)$, cuja função de onda $|X\rangle$ de energia $E_{X}$ foi formulada na equação (4.104). Dessa maneira, a função de onda total do estado final será descrita como

$$
\varphi_{f}\left(\boldsymbol{r}_{1}, \ldots, \boldsymbol{r}_{n}\right)=\left|{ }^{7} F_{J M} ; X\right\rangle
$$

e a energia total do estado final é

$$
E_{J X}=E_{J}+E_{X}
$$

Substituindo os estados inicial e final e as respectivas energias na expressão para o coeficiente de absorção da equação (4.117), obtemos

$$
\alpha(\hbar \omega)=\frac{\pi N e^{2} \hbar \omega}{c \epsilon_{0} \hbar} \sum_{J, M, X}\left|\left\langle{ }^{8} S_{\frac{7}{2}}\left|\hat{\boldsymbol{\xi}} \cdot \sum_{j=1}^{n} \boldsymbol{r}_{j}\right|{ }^{7} F_{J M} ; X\right\rangle\right|^{2} \delta\left(E_{J}+E_{X}-\hbar \omega\right)
$$

Os elementos de matriz da transição na equação (4.121) envolvem a transição por dipolo elétrico de sete elétrons, o que torna a obtenção dos elementos de matriz na equação (4.121) um problema bastante complicado. Tentaremos simplificar o cálculo desses elementos de matriz, onde será mostrado que os elementos de matriz de sete elétrons podem ser reduzidos a elementos de matriz de único elétron.

Utilizando os coeficiente de Clebsch-Gordan, cuja definição pode ser encontrada, por exemplo, na referência [79], podemos representar o estado ${ }^{7} F_{J M}$ numa base de autoestados de spin definido como

$$
\begin{array}{r}
\left|{ }^{7} F_{J M}\right\rangle=|L=3, S=3, J, M\rangle= \\
=\sum_{M_{L}=-L}^{L} \sum_{M_{S}=-S}^{S}\left(J M \mid L=3, S=3, M_{L}, M_{S}\right)\left|L=3, S=3, M_{L}, M_{S}\right\rangle
\end{array}
$$

o que substituído na equação (4.121), onde especificamos os números quânticos do 
estado $\left|{ }^{8} S_{\frac{7}{2}}\right\rangle$, resulta em

$$
\begin{aligned}
\alpha(\hbar \omega) & =\frac{\pi N e^{2} \hbar \omega}{c \epsilon_{0} \hbar} \sum_{J, M, X} \sum_{M_{L}} \sum_{M_{S}}\left(J M \mid L=3, S=3, M_{L}, M_{S}\right)^{2} \delta\left(E_{J}+E_{X}-\hbar \omega\right) \times \\
& \times\left|\left\langle L=0, S=\frac{7}{2}, M_{L}=0, M_{S}=\frac{7}{2}\left|\hat{\boldsymbol{\xi}} \cdot \sum_{j=1}^{n} \boldsymbol{r}_{j}\right| L=S=3, M_{L}, M_{S} ; X\right\rangle\right|^{2}
\end{aligned}
$$

Separando as partes espacial e de spin do estado $|X\rangle$, podemos descrevê-lo por uma função de onda $\left|X, m_{s}\right\rangle$, onde $X$ agora representa a parte espacial de um dos três orbitais $5 d\left(t_{2 g}\right)$ e $m_{s}$ pode assumir os valores $\pm 1 / 2$, de forma que a soma em $X$ no coeficiente de absorção (4.123) é substituída por uma soma em $X$ (espacial) e uma soma em $m_{s}$

$$
\begin{gathered}
\alpha(\hbar \omega)=\frac{\pi N e^{2} \hbar \omega}{c \epsilon_{0} \hbar} \sum_{J, M, X, m_{S}} \sum_{M_{L}} \sum_{M_{S}}\left(J M \mid L=3, S=3, M_{L}, M_{S}\right)^{2} \delta\left(E_{J}+E_{X_{i}}-\hbar \omega\right) \times \\
\times\left|\left\langle L=0, S=\frac{7}{2}, M_{L}=0, M_{S}=\frac{7}{2}\left|\hat{\boldsymbol{\xi}} \cdot \sum_{j=1}^{n} \boldsymbol{r}_{j}\right| L=S=3, M_{L}, M_{S} ; X, m_{S}\right\rangle\right|^{2}
\end{gathered}
$$

Como o operador $\sum_{j} \boldsymbol{r}_{j}$ não atua sobre o spin, os valores dos spins do estado inicial $S=M_{S}=7 / 2$ tem de ser preservados e portanto os únicos elementos de matriz nãonulos serão aqueles para os quais $M_{S}=3$ e $m_{s}=1 / 2$, de forma que os somatórios em $M_{S}$ e $m_{s}$ da equação (4.124) podem ser eliminados, resultando em

$$
\begin{array}{r}
\alpha(\hbar \omega)=\frac{\pi N e^{2} \hbar \omega}{c \epsilon_{0} \hbar} \sum_{J, M, X} \sum_{M_{L}}\left(J M \mid L=3, S=3, M_{L}, M_{S}=3\right)^{2} \delta\left(E_{J}+E_{X}-\hbar \omega\right) \times \\
\times\left|\left\langle L=0, S=\frac{7}{2}, M_{L}=0, M_{S}=\frac{7}{2}\left|\hat{\xi} \cdot \sum_{j=1}^{n} \boldsymbol{r}_{j}\right| L=S=3, M_{L}, M_{S}=3 ; X, m_{S}=\frac{1}{2}\right\rangle\right|^{2}
\end{array}
$$

Mas os coeficientes de Clebsh-Gordan não se anulam apenas se $M_{L}+M_{S}=M$, portanto $M_{L}=M-3$ e o somatório em $M_{L}$ também pode ser eliminado. Fazendo essa eliminação e omitindo os rótulos dos números quânticos nos coeficientes de Clebsh-Gordan, que a partir de agora permanecem fixos, obtemos

$$
\begin{gathered}
\alpha(\hbar \omega)=\frac{\pi N e^{2} \hbar \omega}{c \epsilon_{0} \hbar} \sum_{J, M, X}(J M \mid 3,3, M-3,3)^{2} \delta\left(E_{J}+E_{X}-\hbar \omega\right) \times \\
\times\left|\left\langle L=M_{L}=0, S=M_{S}=\frac{7}{2}\left|\hat{\boldsymbol{\xi}} \cdot \sum_{j=1}^{n} \boldsymbol{r}_{j}\right| L=S=M_{S}=3, M_{L}=M-3 ; X, m_{S}=\frac{1}{2}\right\rangle\right|^{2}(4
\end{gathered}
$$


Para cada um dos três possíveis $|X\rangle$, o elemento de matriz em (4.126) representa um entre sete possíveis, ilustrados na figura 4.7.

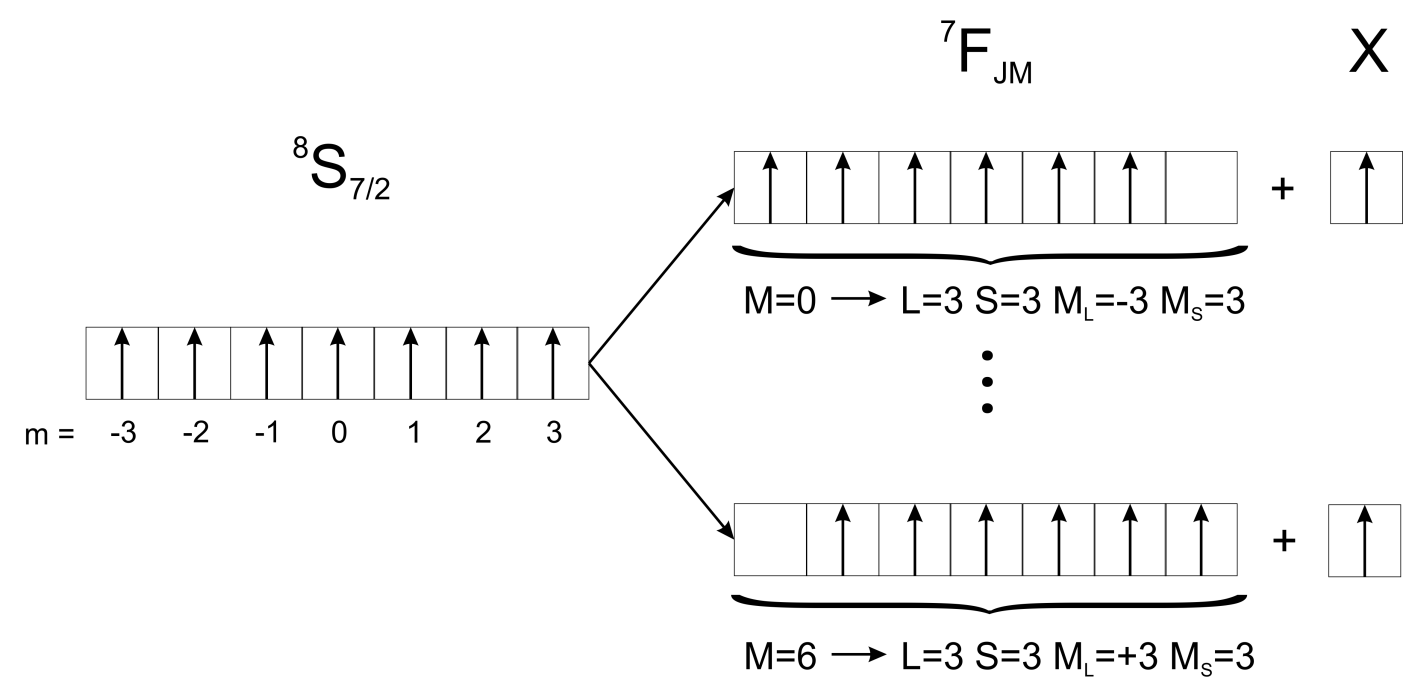

Figura 4.7: Diagrama representando as possíveis transições $4 f^{7} \rightarrow 4 f^{6} X$.

Do diagrama da figura 4.7 fica evidente que a cada elemento de matriz de sete elétrons da equação (4.126), corresponde um elemento de matriz de um elétron. Por exemplo, no primeiro elemento representado, que corresponde a $M=0$, apenas um elétron muda de estado, de $4 f, m=3$ para $X$. Consequentemente

$$
\begin{gathered}
\left\langle L=M_{L}=0, S=M_{S}=\frac{7}{2}\left|\hat{\boldsymbol{\xi}} \cdot \sum_{j=1}^{n} \boldsymbol{r}_{j}\right| L=S=M_{S}=3, M_{L}=M-3 ; X, m_{S}=\frac{1}{2}\right\rangle= \\
=\left\langle 4 f, m=3-M, m_{S}=\frac{1}{2} \hat{\boldsymbol{\xi}} \cdot \boldsymbol{r} \mid X, m_{S}=\frac{1}{2}\right\rangle \underbrace{=}_{\left\langle m_{s} \mid m_{s}\right\rangle=1}\langle 4 f, m=3-M|\hat{\boldsymbol{\xi}} \cdot \boldsymbol{r}| X\rangle
\end{gathered}
$$

$\log \mathrm{O}$

$$
\begin{gathered}
\alpha(\hbar \omega)=\frac{\pi N e^{2} \hbar \omega}{c \epsilon_{0} \hbar} \sum_{J, M, X}(J M \mid 3,3, M-3,3)^{2} \times \\
\times|\langle 4 f, m=3-M|\hat{\boldsymbol{\xi}} \cdot \boldsymbol{r}| X\rangle|^{2} \delta\left(E_{J}+E_{X}-\hbar \omega\right)
\end{gathered}
$$

Agora usaremos a parte espacial do estado $|X\rangle$, que foi determinado na equação (4.104) como

$$
|X(\boldsymbol{r})\rangle=\frac{1}{\sqrt{\mathcal{N}}} \sum_{m^{\prime}=-2}^{2} B\left(X, m^{\prime}\right) \Psi_{5,2, m^{\prime}}(\boldsymbol{r})
$$


onde $\mathcal{N}$ é o número de sítios da rede no volume de Born-Kárman e $B(X, m)$ são os coeficientes da combinação linear das funções atômicas $5 d\left(t_{2 g}\right)$, que foram calculados e apresentados na tabela 4.1 da página 104. Como a energia do estado $X$ depende de $\boldsymbol{k}$, devemos substituir

$$
\sum_{X}=\sum_{k}
$$

mas vamos ignorar a dispersão $E_{X}(\boldsymbol{k})$, de forma que $\sum_{k}=\mathcal{N}$, uma vez que o número de pontos distintos na zona de Brillouin é igual ao número de sítios da rede no período de Born-Kárman. A largura não-nula da faixa de condução pode ser incorporada no coeficiente de absorção substituindo a função $\delta(E)$ por uma função $g(E)$, de largura à meia altura igual à largura da faixa de condução. Com essas considerações, a substituição de (4.129) na equação (4.128) resulta em

$$
\begin{array}{r}
\alpha(\hbar \omega)=\frac{\pi N e^{2} \hbar \omega}{c \epsilon_{0} \hbar} \sum_{J, M} \sum_{m^{\prime}=-2}^{2} B^{2}\left(X, m^{\prime}\right)(J M \mid 3,3, M-3,3)^{2} \times \\
\left|\left\langle\Psi_{4,3, m=3-M}|\hat{\boldsymbol{\xi}} \cdot \boldsymbol{r}| \Psi_{5,2, m^{\prime}}\right\rangle\right|^{2} g\left(E_{J}+E_{X}-\hbar \omega\right)
\end{array}
$$

É importante notar que as funções de onda na equação (4.131) são formuladas em referenciais distintos: a função de onda $\Psi_{4,3, m}(\boldsymbol{r})$ está definida num sistema de coordenadas $O_{4 f} \equiv\left\{\hat{x}_{4 f}, \hat{y}_{4 f}, \hat{z}_{4 f}\right\}$, onde o eixo $\hat{z}_{4 f}$ é o eixo de quantização do momento angular do estado $4 f$; a função $\Psi_{5,2, m^{\prime}}(\boldsymbol{r})$ está definida num outro referencial $O_{5 d} \equiv\left\{\hat{x}_{5 d}, \hat{y}_{5 d}, \hat{z}_{5 d}\right\}$, onde $\hat{z}_{5 d}$ é a direção $z$ do cristal, que pode ser a direção [0,0,1], ou qualquer outra equivalente. O mesmo vale para o vetor de polarização da luz, que é definido num referencial $O_{\phi} \equiv\left\{\hat{x}_{\phi}, \hat{y}_{\phi}, \hat{z}_{\phi}\right\}$, onde $\hat{z}_{\phi}$ é a direção de propagação da luz. Entretanto, para que os elementos de matriz em (4.131) sejam calculados, é necessário que os termos envolvidos sejam descritos no mesmo referencial. Uma maneira de conseguir isso é descrever as funções de onda $\Psi_{4,3, m}(\boldsymbol{r})$ e $\Psi_{5,2, m^{\prime}}(\boldsymbol{r})$ no referencial do fóton, o que pode ser feito através do método que será descrito na seção seguinte. 


\section{Ângulos de Euler e funções de Wigner}

Para descrever as funções de onda $\Psi_{4,3, m}(\boldsymbol{r})$ e $\Psi_{5,2, m^{\prime}}(\boldsymbol{r})$ no referencial do fóton, primeiramente vamos notar que dois sistemas de coordenadas ortogonais distintos podem ser relacionados através de uma combinação de no máximo três rotações. Ou seja, aplicando três rotações em um referencial ortogonal qualquer, é possível torná-lo coincidente com qualquer outro referencial ortogonal. Os ângulos referentes a essas rotações são chamados ângulos de Euler e serão denotados por $(\alpha, \beta, \gamma)$.

Diversas sequências de rotações podem levar ao mesmo resultado, entretanto existem algumas convenções adotadas por muitos autores. Uma delas, a chamada "convenção-y", é definida da seguinte maneira: a primeira rotação pelo ângulo $\alpha$ $(0 \leq \alpha \leq 2 \pi)$ é realizada em torno do eixo $z$; em seguida faz-se a rotação por $\beta$ $(0 \leq \beta \leq \pi)$ ao redor do novo eixo y e por fim a rotação por $\gamma(0 \leq \gamma \leq 2 \pi)$ é feita ao redor do eixo $z$ final. A figura 4.8 ilustra essas operações.

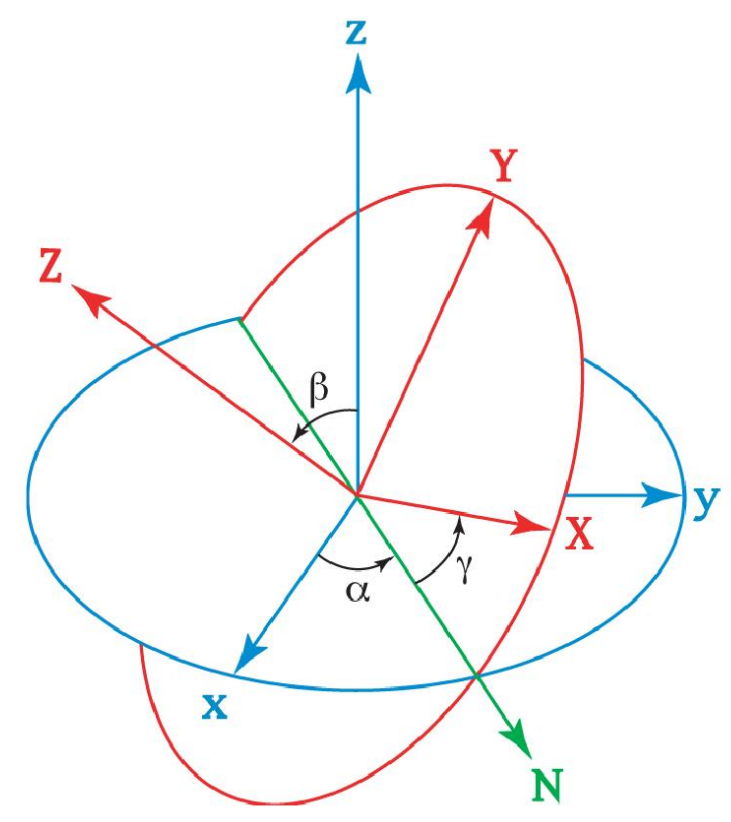

Figura 4.8: As três rotações pelos ângulos de Euler $\alpha, \beta$ e $\gamma$ que levam o referencial $x, y, z$ (em azul) para o referencial $X, Y, Z$ (em vermelho). A linha verde é a chamada linha de nodos.

Para um autoestado de um operador momento angular $j^{2}$, denotado por $|j m\rangle$, têm 
validade as relações

$$
\boldsymbol{j}^{2}|j m\rangle=\hbar^{2} j(j+1)|j m\rangle \text { e } \boldsymbol{j}_{z}|j m\rangle=\hbar m|j m\rangle
$$

Para esse autoestado é possível definir um operador de rotação $\mathcal{R}$, que comuta com $\boldsymbol{j}^{2}$, de forma que quando aplica-se $\mathcal{R}$ sobre $|j m\rangle$, obtém-se um estado de mesmo autovalor $j$, mas com autovalores de $j_{z}$ diversos, os quais denotaremos por $m^{\prime}$. Como o operador de rotação comuta com $\boldsymbol{j}^{2}$ [37], ambos estados são autofunções de $\boldsymbol{j}^{2}$, o que permite que descrevamos o estado $|j m\rangle$ no referencial rotacionado como uma combinação linear de autoestados $\left|j m^{\prime}\right\rangle$

$$
\mathcal{R}|j m\rangle=\sum_{m^{\prime}}\left|j m^{\prime}\right\rangle\left\langle j m^{\prime}|\mathcal{R}| j m\right\rangle
$$

O mesmo vale para três rotações sucessivas

$$
\mathcal{R}(\alpha, \beta, \gamma)|j m\rangle=\sum_{m^{\prime}}\left|j m^{\prime}\right\rangle\left\langle j m^{\prime}|\mathcal{R}(\alpha, \beta, \gamma)| j m\right\rangle
$$

onde os coeficiente da combinação linear são as chamadas funções de Wigner, denotadas por

$$
\left\langle j m^{\prime}|\mathcal{R}(\alpha, \beta, \gamma)| j m\right\rangle=\mathcal{D}_{m m^{\prime}}^{(j)}(\alpha, \beta, \gamma)
$$

Na referência [78], podemos encontrar a fórmula para as funções de Wigner, as quais são fornecidas na forma transposta

$$
\mathcal{D}_{m^{\prime} m}^{(j)}(\alpha, \beta, \gamma)=e^{\imath m^{\prime} \gamma} d_{m^{\prime}, m}^{j}(\beta) e^{\imath m \alpha}
$$

onde as funções $d_{m^{\prime} m}^{j}(\beta)$ são dadas por

$$
d_{m^{\prime} m}^{j}(\beta)=\sqrt{\frac{\left(j+m^{\prime}\right) !\left(j-m^{\prime}\right) !}{(j+m) !(j-m) !}} \cos ^{m^{\prime}+m}\left(\frac{\beta}{2}\right) \operatorname{sen}^{m^{\prime}-m}\left(\frac{\beta}{2}\right) P_{j-m^{\prime}}^{m^{\prime}-m, m^{\prime}+m}(\cos \beta)
$$

onde $P_{j-m^{\prime}}^{m^{\prime}-m, m^{\prime}+m}(\cos \beta)$ são os polinômios de Jacobi. Para inverter a ordem de $m^{\prime} m$ para $m m^{\prime}$ podemos utilizar a relação

$$
d_{m m^{\prime}}^{j}(\beta)=(-1)^{m-m^{\prime}} d_{m^{\prime} m}^{j}(\beta)
$$


encontrada na pg. 60 da referência [80], de forma a obtermos $\mathcal{D}_{m m^{\prime}}^{(j)}(\alpha, \beta, \gamma)$. É importante notar que para $\beta=0$ e $\beta=\pi$, as funções $d_{m^{\prime} m}^{j}(\beta)$ têm uma indeterminação quando $m \pm m^{\prime}<0$. Nesses casos podemos utilizar as relações

$$
d_{m^{\prime} m}^{j}( \pm \pi)=(-1)^{j \pm m} \delta_{m^{\prime},-m}
$$

e

$$
d_{m^{\prime} m}^{j}(\pi+\beta)=(-1)^{j-m^{\prime}} d_{-m^{\prime} m}^{j}(\beta) \underbrace{\rightarrow}_{\beta=-\pi} d_{m^{\prime} m}^{j}(0)=(-1)^{j-m} d_{-m^{\prime} m}^{j}(-\pi)=\delta_{m^{\prime}, m}
$$

também encontradas na referência [80].

Com isso podemos descrever as funções de onda $\Psi_{4,3, m}(\boldsymbol{r})$ e $\Psi_{5,2, m^{\prime}}(\boldsymbol{r})$, que são autofunções de seus operadores momento angular, no referencial do fóton, utilizando a equação (4.133), desde que conheçamos os ângulos de Euler que relacionam os referenciais $\mathrm{O}_{4 f}$ e $\mathrm{O}_{5 d}$ com o referencial $O_{\phi}$.

Para determinar esses ângulos, vamos utilizar um método geral que fornece os ângulos de Euler que relacionam um referencial $x, y, z \operatorname{com}$ outro referencial $X, Y, Z$. Esse método utiliza-se do plano complexo para evitar a inversão de funções trigonométricas e funciona da seguinte maneira: inicialmente calcula-se o vetor $N \equiv \boldsymbol{z} \times \boldsymbol{Z}$, que é chamado vetor da linha de nodos. Em seguida, definem-se três números complexos,

$$
\begin{aligned}
\tilde{u} & =\boldsymbol{N} \cdot \boldsymbol{x}+\imath \boldsymbol{N} \cdot \boldsymbol{y} \\
\tilde{v} & =\boldsymbol{z} \cdot \boldsymbol{Z}+\imath \mathbf{N} \\
\tilde{\boldsymbol{w}} & =\boldsymbol{N} \cdot \boldsymbol{X}+\imath \mathbf{N} \cdot \mathbf{Y}
\end{aligned}
$$

onde $N=|N|$, do quais é possível obter os ângulos de Euler como

$$
\begin{array}{r}
\alpha=\arg \tilde{u} \\
\beta=\arg \tilde{\mathcal{v}} \\
\gamma=-\arg \tilde{\mathcal{w}}
\end{array}
$$

Contudo este método é definido na "convenção- $x$ " das rotações de Euler, que é muito similar à "convenção-y"; a única diferença é que a segunda rotação é efetuada 
ao redor da nova direção $x$ obtida após a primeira rotação. Como as funções de Wigner foram definidas para ângulos de Euler na "convenção-y", levamos em conta que devido à similaridade entre as duas convenções, é possível exprimir os ângulos de Euler na "convenção-y" a partir dos ângulos de Euler na "convenção-x", com uma simples translação de $-\pi / 2$ em $\alpha$ e $+\pi / 2$ em $\gamma$, sendo que o ângulo $\beta$ permanece inalterado. Com isso podemos determinar os ângulos de Euler na "convenção-y" como

$$
\begin{aligned}
& \alpha=\arg (\tilde{u})-\pi / 2 \\
& \beta=\arg (\tilde{\mathcal{v}}) \\
& \gamma=-\arg (\tilde{\mathfrak{w}})+\pi / 2
\end{aligned}
$$

A seguir serão exemplificados os procedimentos da determinação dos ângulos de Euler e da descrição das funções de onda em referenciais rotacionados, para casos de interesse no cálculo do coeficiente de absorção dos EuX.

Exemplo 1 - Determinação dos ângulos de Euler -

No cálculo de cada elemento de matriz é necessário conhecer os ângulos de rotação que relacionam os referenciais nos quais são descritas as funções de onda $4 f$ e $5 d$ com o referencial do fóton. O vetor de polarização complexo, $\hat{\xi}$, foi definido para uma luz que se propaga na direção $z$. Os eixos $x$ e $y$ de um feixe de luz circularmente polarizada não são definidos, de forma que existe uma arbitrariedade na escolha desse referencial. Quando a polarização da luz é linear, definimos a direção $x$ como a direção de polarização.

O referencial onde são descritas as funções de onda $4 f$ dos átomos de Eu é determinado pelo eixo de quantização do spin. Em campo nulo os spins da rede distribuem-se em domínios magnéticos, orientados de acordo com a estrutura cristalina do material. Quando há um campo magnético aplicado, os spins da rede alinham-se para o sentido contrário ao do campo magnético, de forma que o eixo $z_{4 f}$ é determinado pela direção do campo magnético. Vamos supor a situação onde há um campo magnético aplicado na direção $x \phi$ do referencial do fóton. Neste caso, a direção $z_{4 f}$ é a direção $-x_{\phi}$ do referencial do fóton. Os eixos $x_{4 f}$ e $y_{4 f}$ não são definidos, então podemos defini-los arbitrariamente, desde que formem um conjunto ortogonal dextrogiro com $z_{4 f}$. Uma 
possível escolha é $x_{4 f}=-z_{\phi}$ e $y_{4 f}=-y_{\phi}$, que corresponde ao representado na figura 4.9 , onde fica evidente que ao aplicar-se sobre o referencial $O_{4 f}$ uma rotação de um ângulo $\alpha=\pi$ ao redor do eixo $z_{4 f}$ e de um ângulo $\beta=\pi / 2$ ao redor do novo eixo $y_{4 f}$, este referencial coincide com o referencial do fóton.

a)

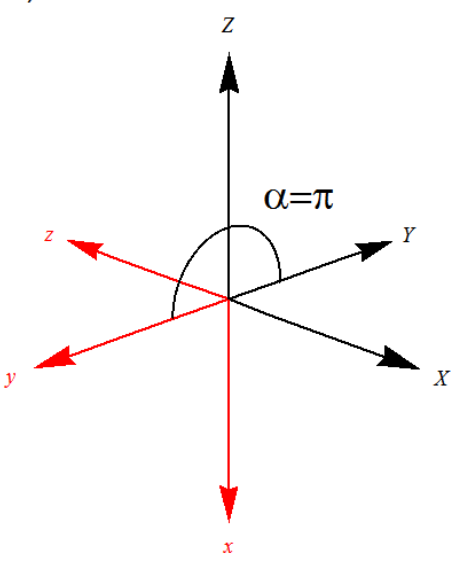

b)

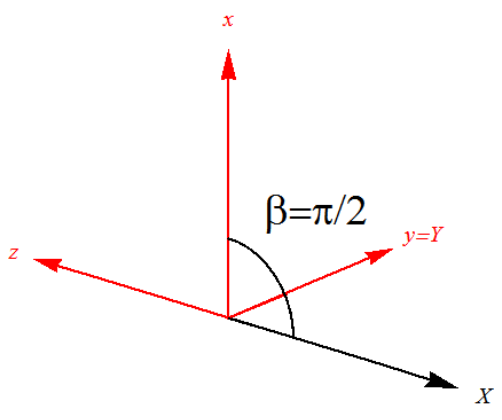

c)

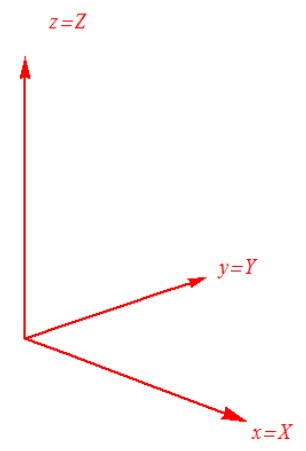

Figura 4.9: Rotações de Euler com a) $\alpha=\pi$, b) $\beta=\pi / 2$ e c) $\gamma=0$.

Utilizando o método das equações (4.143) e (4.149) é possível chegarmos ao mesmo resultado, como será demonstrado a seguir: no referencial do fóton temos os sistemas de coordenadas $O_{\phi}=\{(1,0,0),(0,1,0),(0,0,1)\}$ e $O_{4 f}=\{(0,0,-1),(0,-1,0),(-1,0,0)\}$, de forma que

$$
\begin{aligned}
\boldsymbol{N} & =z_{4 f} \times z_{\phi}=(0,1,0) \\
\tilde{u} & =\boldsymbol{N} \cdot \boldsymbol{x}_{4 f}+\imath \mathbf{N} \cdot \boldsymbol{y}_{4 f}=-\imath \\
\tilde{v} & =z_{4 f} \cdot \boldsymbol{z}_{\phi}+\imath N=\imath \\
\tilde{w} & =\boldsymbol{N} \cdot \boldsymbol{x}_{\phi}+\imath \mathbf{N} \cdot \mathbf{y}_{\phi}=\imath
\end{aligned}
$$

substituindo $\tilde{u}, \tilde{v}$ e $\tilde{w}$ na equação (4.149) obtemos

$$
\begin{aligned}
& \alpha=\arg (-\imath)-\pi / 2=-\pi \rightarrow \alpha=\pi \\
& \beta=\arg (\imath)=\pi / 2 \\
& \gamma=-\arg (\imath)+\pi / 2=0
\end{aligned}
$$


Agora devemos encontrar os ângulos que relacionam o referencial onde estão definidas as funções de onda $5 d$, que é determinado pela estrutura cristalina, com o referencial do fóton. Se o feixe de luz incide perpendicularmente à superfície da amostra, a direção $z_{\phi}$ é orientada paralelamente à direção de crescimento epitaxial, que em nossas amostras caso é a direção de índices de Miller [111], definida no sistema de coordenadas do cristal. Para polarização linear da luz devemos definir também os eixos $x_{\phi}$ e $y_{\phi}$, para levar em conta a orientação com o campo magnético. Vamos supor que o campo magnético seja aplicado na direção cristalina de índices de Miller [112̄] e definir essa como a direção $x_{\phi}$, de forma que a orientação do campo é paralela à direção de polarização da luz. Agora o eixo $y_{\phi}$ fica determinado por $y_{\phi}=z_{\phi} \times x_{\phi}=(-3,3,0)$. Portanto $O_{\phi}=\{(1,1,-2),(-3,3,0),(1,1,1)\}$.

O referencial do cristal, no qual são descritas as funções de onda $5 d$, é definido pelos eixos da estrutura cúbica, $O_{5 d}=\{(1,0,0),(0,1,0),(0,0,1)\}$. Empregando as equações (4.143) e (4.149) para os referenciais $O_{\phi}$ e $O_{5 d}$ obtemos

$$
\begin{aligned}
& \alpha=\pi / 4 \\
& \beta=\tan ^{-1}(\sqrt{2}) \\
& \gamma=0
\end{aligned}
$$

o que está representado na figura 4.10 .
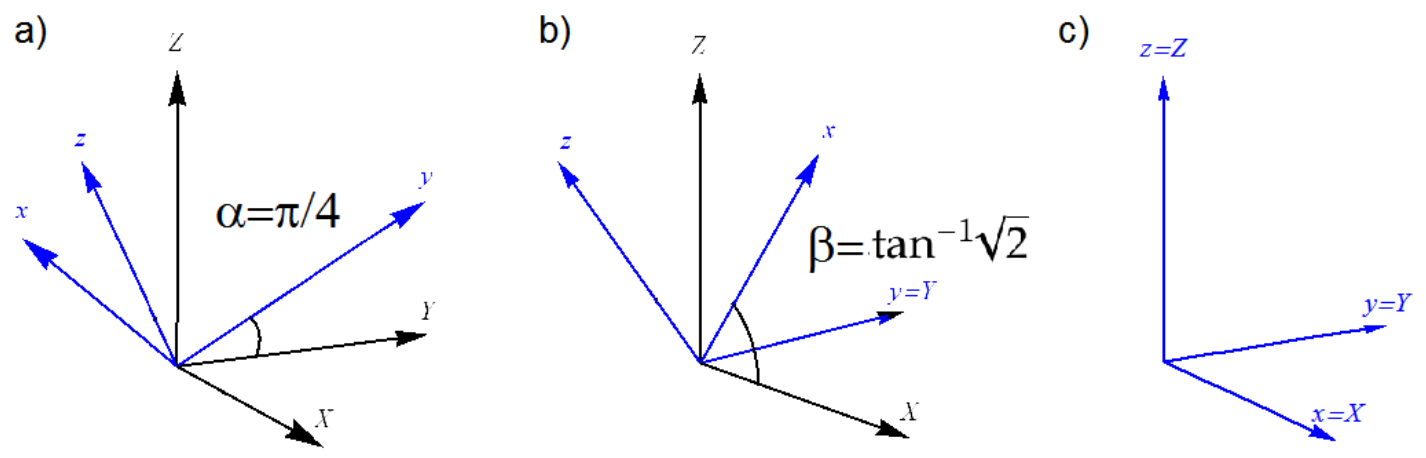

Figura 4.10: Rotações de Euler a) $\alpha=\pi / 4, b) \beta=\tan ^{-1}(\sqrt{2})$ e c) $\gamma=0$.

Exemplo 2 - Matrizes de Wigner - Seja um orbital $f$, descrito por autoestados de 
$j^{2}$ dados por $|j=3, m\rangle$ no referencial $O$. Para descrever esse orbital em um outro referencial $O^{\prime}$, relacionado com $O$ por três rotações nos ângulos $(\alpha, \beta, \gamma)$ definidas na "convenção-y", podemos utilizar a equação (4.133)

$$
\mathcal{R}(\alpha, \beta, \gamma)|j=3, m\rangle=\sum_{m^{\prime}=-3}^{3}\left|j m^{\prime}\right\rangle\left\langle j m^{\prime}|\mathcal{R}(\alpha, \beta, \gamma)| j m\right\rangle=\sum_{m^{\prime}=-3}^{3} \mathcal{D}_{m m^{\prime}}^{3}\left|j=3, m^{\prime}\right\rangle
$$

Para cada valor de $m$, existem $2(j+1)$ coeficientes da combinação linear, um para cada $m^{\prime}$. Os coeficientes para cada par $\left(m, m^{\prime}\right)$ podem ser agrupados em uma matriz $m \times m^{\prime}$ de ordem $2(j+1)$, chamada matriz de Wigner, cujos elementos são as funções de Wigner $\mathcal{D}_{m m^{\prime}}^{j}(\alpha, \beta, \gamma)$. Vamos supor que os referenciais $O$ e $O^{\prime}$ sejam relacionados por rotações nos ângulos de Euler $\alpha=\pi, \beta=\pi / 2$ e $\gamma=0$. Utilizando as equações (4.136) a (4.139) podemos calcular os elementos de matriz com $j=3$, os quais para os ângulos escolhidos resultam na matriz

$$
\mathcal{M}_{m m^{\prime}}^{(3)}\left(\pi, \frac{\pi}{2}, 0\right)=\left(\begin{array}{ccccccc}
\frac{1}{8} & \sqrt{\frac{3}{32}} & -\sqrt{\frac{15}{64}} & \sqrt{\frac{5}{16}} & -\sqrt{\frac{15}{64}} & \sqrt{\frac{3}{32}} & -\frac{1}{8} \\
\sqrt{\frac{3}{32}} & -\frac{1}{2} & \sqrt{\frac{5}{32}} & 0 & -\sqrt{\frac{5}{32}} & \frac{1}{2} & -\sqrt{\frac{3}{32}} \\
-\sqrt{\frac{15}{64}} & \sqrt{\frac{5}{32}} & \frac{1}{8} & -\sqrt{\frac{3}{16}} & \frac{1}{8} & \sqrt{\frac{5}{32}} & -\sqrt{\frac{15}{64}} \\
\sqrt{\frac{5}{16}} & 0 & -\sqrt{\frac{3}{16}} & 0 & \sqrt{\frac{3}{16}} & 0 & -\sqrt{\frac{5}{16}} \\
-\sqrt{\frac{15}{64}} & -\sqrt{\frac{5}{32}} & \frac{1}{8} & \sqrt{\frac{3}{16}} & \frac{1}{8} & -\sqrt{\frac{5}{32}} & -\sqrt{\frac{15}{64}} \\
\sqrt{\frac{3}{32}} & \frac{1}{2} & \sqrt{\frac{5}{32}} & 0 & -\sqrt{\frac{5}{32}} & -\frac{1}{2} & -\sqrt{\frac{3}{32}} \\
-\frac{1}{8} & -\sqrt{\frac{3}{32}} & -\sqrt{\frac{15}{64}} & -\sqrt{\frac{5}{16}} & -\sqrt{\frac{15}{64}} & -\sqrt{\frac{3}{32}} & -\frac{1}{8}
\end{array}\right)
$$

Com isso, podemos denotar o coeficiente de absorção em sua forma mais geral, incluindo as funções de Wigner que permitem que todos os elementos envolvidos no cálculo dos elementos de matriz da transição sejam descritos em um mesmo referencial. A partir da expressão para o coeficiente de absorção (4.131), substituímos as funções de onda $\Psi_{4,3, m}$ por $\sum_{m_{1}} \mathcal{D}_{m m_{1}}^{(3)}(\alpha, \beta, \gamma) \Psi_{4,3, m_{1}}$ e $\Psi_{5,2, m^{\prime}}$ por $\sum_{m_{2}} \mathcal{D}_{m m_{2}}^{(2)}(\alpha, \beta, \gamma) \Psi_{5,2, m_{2}}$ para obtermos

$$
\begin{array}{r}
\alpha(\hbar \omega)=\frac{\pi N e^{2} \hbar \omega}{c \epsilon_{0} \hbar} \sum_{J, M} \sum_{m_{1}=-3}^{3} \sum_{m_{2}=-2}^{3} B^{2}\left(X, m_{2}\right)(J M \mid 3,3, M-3,3)^{2} \times \\
\times\left|D_{m m_{1}}^{(3)}(\alpha, \beta, \gamma) D_{m m_{2}}^{(2)}(\alpha, \beta, \gamma)\left\langle\Psi_{4,3, m_{1}}|\hat{\boldsymbol{\xi}} \cdot \boldsymbol{r}| \Psi_{5,2, m_{2}}\right\rangle\right|^{2} g\left(E_{J}+E_{X}-\hbar \omega\right)
\end{array}
$$




\section{Geometrias Faraday e Voigt}

As denominações Faraday e Voigt dão-se à orientação de um feixe de luz em relação à orientação do campo magnético aplicado do sistema. Na geometria Faraday, a direção de propagação da luz é paralela à direção do campo magnético, o que está ilustrado na figura 4.11 .

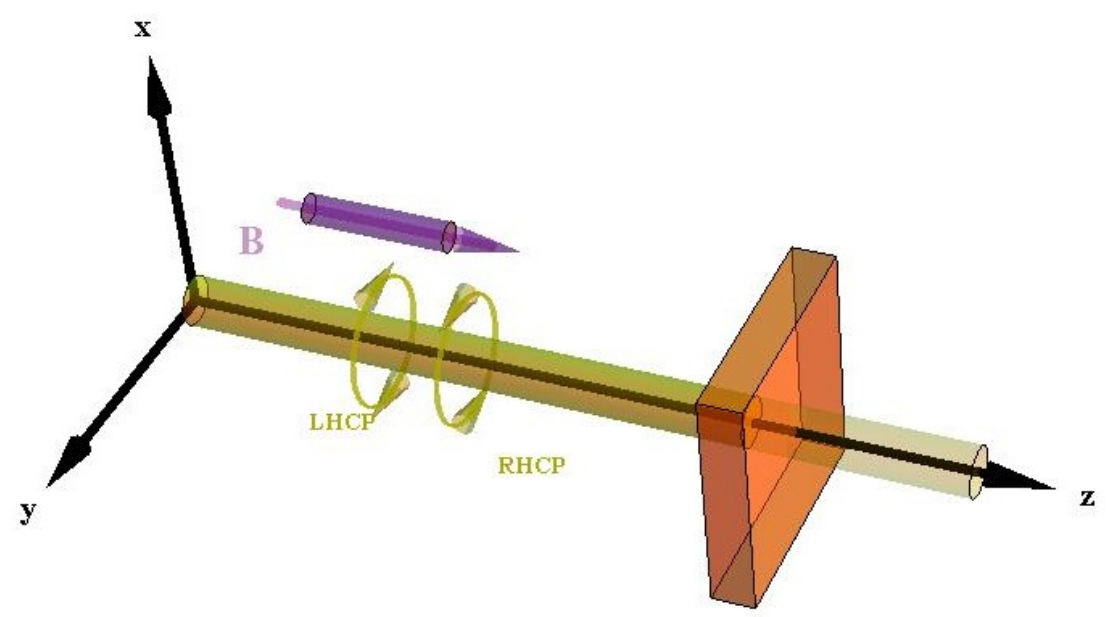

Figura 4.11: Geometria Faraday. O vetor campo elétrico oscila numa direção que gira ao redor da direção de propagação, para a esquerda ou para a direita. O campo magnético é paralelo à direção de propagração.

Na geometria Voigt, a direção de propagação da luz é perpendicular à direção do campo magnético e o vetor campo elétrico, que determina o eixo de polarização da luz, pode ser orientado paralelamente ao campo (configuração \|) ou perpendicularmente ao campo (configuração $\perp$ ), como mostra a figura 4.12 .

Neste trabalho, com a geometria Faraday foi utilizada sempre a polarização circular da luz e na geometria Voigt, a polarização linear da luz. A luz circularmente polarizada carrega momento angular, enquanto que a luz linearmente polarizada tem momento angular nulo. Esse fato implica em diferenças fundamentais entre a absorção nos arranjos Faraday e Voigt, portanto medidas nas duas configurações fornecem informações diferentes sobre o material, como veremos mais adiante na seção 4.1.8. A polarização da luz é expressa no coeficiente de absorção através do vetor de polarização $\hat{\xi}$ em 


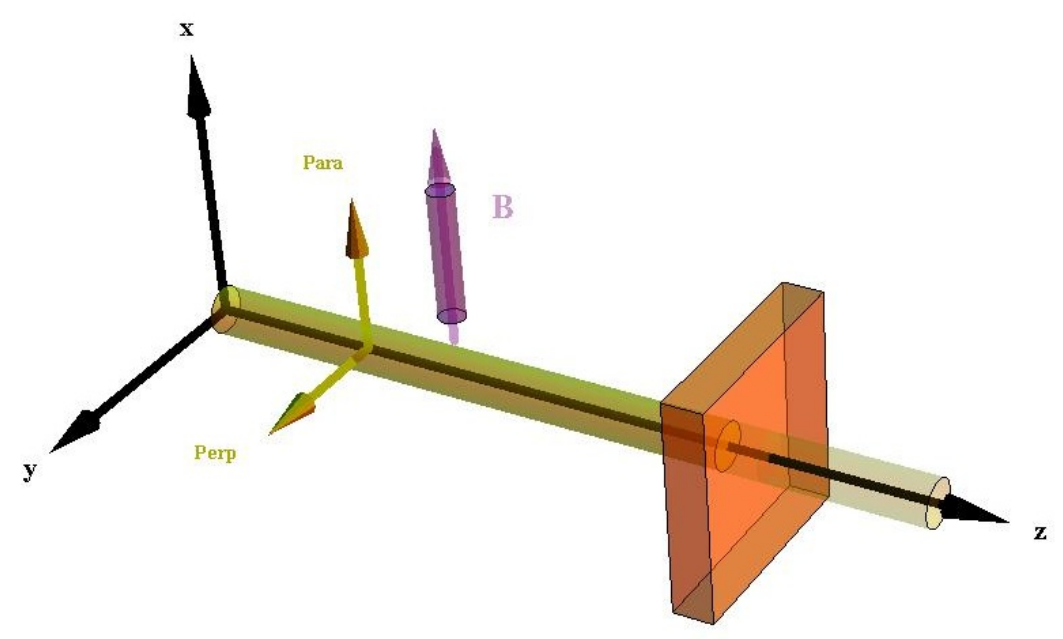

Figura 4.12: Geometria Voigt. O vetor campo elétrico oscila perpendicularmente à direção de propagação, podendo estar alinhado paralelamente o perpendicularmente com o campo magnético.

(4.162), o qual foi deduzido na seção 4.1.2 para as duas polarizações da luz.

\section{Cálculo dos elementos de matriz Faraday e Voigt}

Os elementos de matriz da equação (4.162),

$$
\left\langle\Psi_{4,3, m=3-M}|\hat{\boldsymbol{\xi}} \cdot \boldsymbol{r}| \Psi_{5,2, m^{\prime}}\right\rangle
$$

onde mudamos a notação de $m_{1} \rightarrow m$ e $m_{2} \rightarrow m^{\prime}$, dependem do vetor de polarização $\hat{\xi}$ e por isso são diferentes para as geometrias Faraday e Voigt. Esses elementos podem ser calculados substituindo as formas explícitas das funções de onda, encontradas por exemplo na referência [78] e efetuando as integrações.

Para calcular as integrais (4.163), vamos separar as funções de onda nas componentes radial e angular. As integrais radiais não dependem do número quântico $m$, e como $n$ e $\ell$ são fixos, integração da parte radial resulta em uma constante, a qual denotaremos por $r_{d f}$. A parte angular é descrita pelos harmônicos esféricos $Y_{\ell, m}$ e pode ainda ser separada nas componentes azimutal $I(\phi)$ e polar $I(\theta)$. Como para cada geometria utilizada o vetor de polarização $\hat{\xi}$ diferente, vamos calcular as integrais angulares 
separadamente para as geometria Faraday e Voigt.

As integrais azimutais para a geometria Faraday são

$$
I(\phi)^{+}=\int_{0}^{2 \pi} e^{\imath\left(m^{\prime}-m\right) \phi}(\cos \phi+\imath \operatorname{sen} \phi) d \phi
$$

para a luz RHCP $\left(\sigma^{+}\right)$e

$$
I(\phi)^{-}=\int_{0}^{2 \pi} e^{\imath\left(m^{\prime}-m\right) \phi}(\cos \phi-\imath \operatorname{sen} \phi) d \phi
$$

para a luz $\operatorname{LHCP}\left(\sigma^{-}\right)$.

Na na geometria Voigt, são

$$
I(\phi)^{\|}=\int_{0}^{2 \pi} e^{\imath\left(m^{\prime}-m\right) \phi} \cos \phi d \phi
$$

para o vetor de polarização orientado paralelo ao campo magnético $\left(\sigma^{\|}\right)$e

$$
I(\phi)^{\perp}=\int_{0}^{2 \pi} e^{\imath\left(m^{\prime}-m\right) \phi} \operatorname{sen} \phi d \phi
$$

para o vetor de polarização perpendicular ao campo magnético $\left(\sigma^{\perp}\right)$.

Para efetuar a integração em (4.164), representamos as funções sen $\phi$ e $\cos \phi$ em suas formas exponenciais, o que resulta em

$$
\begin{aligned}
I(\phi)^{+} & =\int_{0}^{2 \pi} e^{\imath\left(m^{\prime}-m\right) \phi}\left(\frac{e^{l \phi}+e^{-\imath \phi}}{2}+\imath \frac{e^{l \phi}-e^{-l \phi}}{2 \imath}\right) d \phi= \\
& =\frac{1}{2}\left[\int_{0}^{2 \pi} e^{\imath\left(m^{\prime}-m+1\right) \phi} d \phi+\int_{0}^{2 \pi} e^{\imath\left(m^{\prime}-m-1\right) \phi} d \phi\right]+ \\
& +\frac{1}{2}\left[\int_{0}^{2 \pi} e^{\imath\left(m^{\prime}-m+1\right) \phi} d \phi-\int_{0}^{2 \pi} e^{\imath\left(m^{\prime}-m-1\right) \phi} d \phi\right]= \\
& =\int_{0}^{2 \pi} e^{\imath\left(m^{\prime}-m+1\right) \phi} d \phi
\end{aligned}
$$

Como a integral de uma função trigonométrica em um período é sempre nula, (4.168) não se anula apenas quando o argumento da exponencial for nulo, o que implica em

$$
\Delta m=m^{\prime}-m+1=0 \rightarrow \Delta m=-1
$$

e nesse caso o resultado da integral é simplesmente

$$
I(\phi)^{+}=2 \pi
$$


Analogamente, obtemos para a equação (4.165)

$$
I(\phi)^{-}=2 \pi
$$

desde que verificada a condição

$$
\Delta m=+1
$$

As integrais $I(\phi)^{\|}$e $I(\phi)^{\perp}$ não se anulam para $\Delta m= \pm 1$ : quando $\Delta m=+1$, temos $I(\phi)^{\|}=\pi$ e $I(\phi)^{\perp}=-\imath \pi$ e quando $\Delta m=-1$, temos $I(\phi)^{\|}=\pi$ e $I(\phi)^{\perp}=\imath \pi$, de forma que

$$
I(\phi)^{\|}=\pi, \Delta m= \pm 1
$$

e

$$
I(\phi)^{\perp}= \begin{cases}-\imath \pi, & \Delta m=+1 \\ \imath \pi, & \Delta m=-1\end{cases}
$$

As condições

$$
\begin{array}{ll}
\Delta m=m^{\prime}-m=-1 & (\mathrm{RHCP}) \\
\Delta m=m^{\prime}-m=+1 & (\mathrm{LHCP}) \\
\Delta m=m^{\prime}-m= \pm 1 & (\|, \perp)
\end{array}
$$

correspondem às regras de seleção das transições de dipolo elétrico para esse sistema.

As integrais polares são iguais em todos os casos e dadas por

$$
I(\theta)=\int_{0}^{2 \pi}\left[Y_{3}^{m}(\cos \theta)\right]^{*} Y_{2}^{m^{\prime}}(\cos \theta) \operatorname{sen}^{2} \theta d \theta
$$

Para efetuar essa integral, substituímos a forma explícita dos harmônicos esféricos, que podem ser encontradas por exemplo na pg. 91 da referência [78].

Como $m=(-3, \ldots,+3)$ e $m^{\prime}=(-2, \ldots,+2)$, as integrais $I(\theta)$ foram calculadas para cada par $\left\{m^{\prime}, m\right\}$, para cada $\hat{\xi}$. Com os resultados de $I(\theta)$, podemos voltar para a integral total

$$
\left\langle\Psi_{4,3, m=3-M}|\hat{\boldsymbol{\xi}} \cdot \boldsymbol{r}| \Psi_{5,2, m^{\prime}}\right\rangle=r_{d f} I(\phi) I(\theta)
$$


e sumarizar os resultados em quatro matrizes $7 \times 5$, uma para cada configuração da transição. São elas

$$
\begin{gathered}
\mathcal{M}_{m, m^{\prime}}^{(+)}=r_{d f}\left(\begin{array}{ccccc}
\sqrt{\frac{6}{7}} & 0 & 0 & 0 & 0 \\
0 & \frac{2}{\sqrt{7}} & 0 & 0 & 0 \\
0 & 0 & 2 \sqrt{\frac{3}{35}} & 0 & 0 \\
0 & 0 & 0 & \sqrt{\frac{6}{35}} & 0 \\
0 & 0 & 0 & 0 & \sqrt{\frac{2}{35}} \\
0 & 0 & 0 & 0 & 0 \\
0 & 0 & 0 & 0 & 0
\end{array}\right) \\
\mathcal{M}_{m, m^{\prime}}^{(-)}=r_{d f}^{(I I)}=\frac{\imath r_{d f}}{\sqrt{2}}\left(\begin{array}{ccccc}
0 & 0 & 0 & 0 & 0 \\
0 & 0 & 0 & 0 & 0 \\
\sqrt{\frac{2}{35}} & 0 & 0 & 0 & 0 \\
0 & \sqrt{\frac{6}{35}} & 0 & 0 & 0 \\
0 & 0 & 2 \sqrt{\frac{3}{35}} & 0 & 0 \\
0 & 0 & 0 & \frac{2}{\sqrt{7}} & 0 \\
0 & 0 & 0 & 0 & \sqrt{\frac{6}{7}}
\end{array}\right) \\
\left.0 \begin{array}{ccccc}
\sqrt{\frac{2}{35}} & 0 & 2 \sqrt{\frac{3}{35}} & 0 & 0 \\
0 & -\sqrt{\frac{6}{35}} & 0 & \sqrt{\frac{6}{35}} & 0 \\
0 & 0 & -2 \sqrt{\frac{3}{35}} & 0 & \sqrt{\frac{2}{35}} \\
0 & 0 & 0 & -\frac{2}{\sqrt{7}} & 0 \\
0 & 0 & 0 & 0 & -\sqrt{\frac{6}{7}}
\end{array}\right)
\end{gathered}
$$




$$
\mathcal{M}_{m, m^{\prime}}^{(\perp)}=\frac{r_{d f}}{\sqrt{2}}\left(\begin{array}{ccccc}
\sqrt{\frac{6}{7}} & 0 & 0 & 0 & 0 \\
0 & \frac{2}{\sqrt{7}} & 0 & 0 & 0 \\
\sqrt{\frac{2}{35}} & 0 & 2 \sqrt{\frac{3}{35}} & 0 & 0 \\
0 & \sqrt{\frac{6}{35}} & 0 & \sqrt{\frac{6}{35}} & 0 \\
0 & 0 & 2 \sqrt{\frac{3}{35}} & 0 & \sqrt{\frac{2}{35}} \\
0 & 0 & 0 & \frac{2}{\sqrt{7}} & 0 \\
0 & 0 & 0 & 0 & \sqrt{\frac{6}{7}}
\end{array}\right)
$$

onde $\mathcal{M}_{m, m^{\prime}}^{(+),(-)}$valem para a geometria Faraday e $\mathcal{M}_{m, m^{\prime}}^{((I),(\perp)}$ para a geometria Voigt.

É interessante notar que essas matrizes estão relacionadas entre si pelas equações

$$
\begin{aligned}
\mathcal{M}_{\|} & =\frac{\mathcal{M}^{+}+\mathcal{M}^{-}}{\sqrt{2}} \\
\mathcal{M}_{\perp} & =\frac{\mathcal{M}^{+}-\mathcal{M}^{-}}{\imath \sqrt{2}}
\end{aligned}
$$

Em termos das matrizes de transição, o coeficiente de absorção (4.162) é dado por

$$
\begin{array}{r}
\alpha(\hbar \omega)=\frac{\pi N e^{2} \hbar \omega}{c \epsilon_{0} \hbar} \sum_{J, M} \sum_{m_{1}=-3}^{3} \sum_{m_{2}=-2}^{2} g\left(E_{J}+E_{X}-\hbar \omega\right) \times \\
\times B^{2}\left(X, m_{2}\right)(J M \mid 3,3, M-3,3)^{2}\left|\mathcal{D}_{m_{1}, m}^{(3)}(\alpha, \beta, \gamma) \mathcal{D}_{m_{2}, m^{\prime}}^{(2)}(\alpha, \beta, \gamma) \mathcal{M}_{m, m^{\prime}}\right|^{2}
\end{array}
$$

onde $\mathcal{M}_{m, m^{\prime}}$ representa a matriz de transição associada a cada geometria (Faraday ou Voigt) e polarização à da luz. Na seção seguinte, a partir da equação (4.186), iremos calcular os espectros de absorção dos EuX para as diferentes configurações possíveis.

\subsubsection{Espectros de absorção dos EuX}

Através de (4.186) é possível calcular os espectros de absorção, definido-se os parâmetros fixos e efetuando todas as somas. Vamos supor que o ordenamento magnético da rede seja AFMII, de forma que na presença de um campo magnético, o elétron fotoexcitado tem sua energia $E_{X}$ reduzida pela interação de exchange de acordo com a equação 
(4.108). Definindo $E(\boldsymbol{k})=0$, a energia $E_{X}=E(\boldsymbol{k})+\Delta E$ fica dada por

$$
E_{X}=-J_{d f} S \cos ^{2} \theta
$$

onde $J_{d f}$ é a constante de exchange $d-f$ e $\theta$ éo ângulo entre o spin do elétron fotoexcitado e os spins da rede, o qual depende do campo magnético de acordo com a equação (4.113)

$$
\cos \theta= \begin{cases}\frac{B}{B_{S A T}}, & B<B_{S A T} \\ 1, & B \geq B_{S A T}\end{cases}
$$

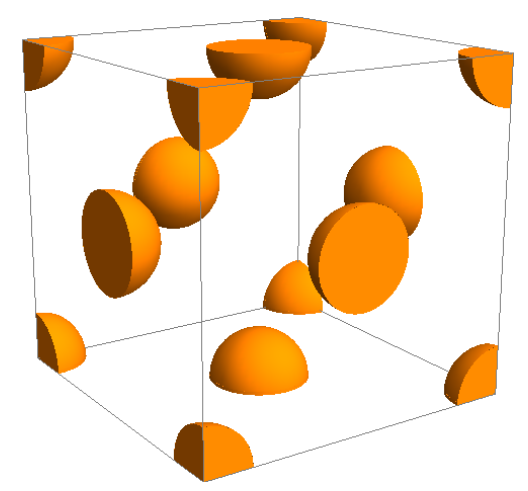

Figura 4.13: $\mathrm{O}$ número total de íons $\mathrm{Eu}^{2+}$ em uma célula primitiva pode ser obtido dividindo cada íon pelo número de células primitivas que o compartilham. Assim, nos vértices somam-se os oito oitavos para contar um íon. Nas faces somam-se três metades, totalizando na estrutura $f c c N=4 / a^{3}$.

A densidade de sítios $N$ pode ser determinada para os $\mathrm{EuX}$ considerando como absorventes apenas os átomos de $\mathrm{Eu}$, os quais são arranjados numa estrutura $f c c$. A figura 4.13 ilustra uma célula primitiva da rede $f c c$, de onde podemos determinar a densidade de sítios como

$$
N=\frac{4}{a^{3}}
$$

onde $a$ é o parâmetro de rede.

Os demais parâmetros utilizados nos cálculos dos espectros de absorção do EuTe foram os mesmos utilizados na referência [28]: $E_{G}=2.321 \mathrm{eV}, r_{d f}=0.18 \AA, \lambda_{4 f}=$ 
$9.6 \mathrm{meV}$ e $J_{d f} S=0.15 \mathrm{eV}$. A função normalizada $g\left(E_{J}+E_{X}-\hbar \omega\right)$ em (4.186) é escolhida como uma distribuição voigtiana com largura a meia altura de $65 \mathrm{meV}$.

Em campo zero, o EuTe tem sua rede dividida em doze domínios magnéticos. Nesta situação o espectro de absorção é dado pela média dos espectros de cada domínio magnético individual, o que resulta nos espectros da figura (4.14), apresentados para as polarizações linear e circular da luz.
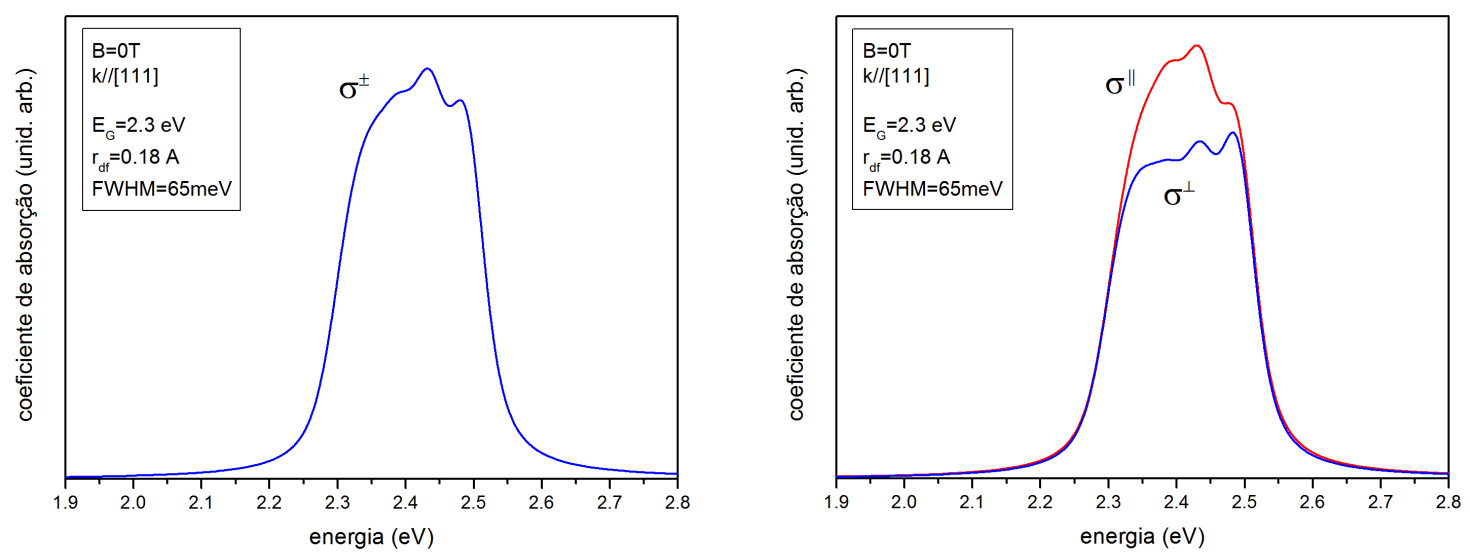

Figura 4.14: Espectros teóricos para EuTe em campo nulo a) com polarização circular da luz e b) com polarização linear da luz.

Notamos na figura (4.14) a) que devido à simetria de inversão, os espectros para as polarizações $\sigma^{ \pm}$são idênticos. Na figura (4.14) b) notamos que os espectros de absorção de luz linearmente polarizada são diferentes para $\sigma^{\|}$e $\sigma^{\perp}$. Em ambos os casos a) e b) fica evidente que a estrutura de domínios impede que apareçam linhas estreitas no espectro de absorção.

Para incluir a presença do campo magnético, notamos que este manifesta-se nos espectros em dois momentos: no deslocamento das linhas de absorção em virtude da interação de exchange $d-f$ e na definição da orientação dos spins da rede, que varia com o campo e define o eixo de quantização dos orbitais $4 f$. Na prática a orientação do campo determina a matriz de Wigner que é utilizada para descrever os orbitais $4 f$ no referencial do fóton, como discutido na seção 4.1.7.

Em campo alto, quando o arranjo de spins é ferromagnético, os spins $4 f$ dos íons 
de európio encontram-se alinhados à direção do campo. Na configuração Faraday, o campo magnético é orientado na direção de propagação da luz, ou seja, a direção $z_{\phi}$ do referencial do fóton, portanto não é necessário efetuar nenhuma rotação do orbital $4 f$. Na configuração Voigt, com o campo orientado no sentido $x_{\phi}$, os spins nos orbitais $4 f$, que definem o eixo $z_{4 f}$, estão alinhados à direção $-x_{\phi}$. Então podemos definir o referencial $O_{4 f}$ no sistema de coordenadas do referencial $O_{\phi}=\{X, Y, Z\}$ como aquele determinado pelos vetores $\{x, y, z\}=\{(0,0,-1),(0,-1,0),(-1,0,0)\}$. Os ângulos de Euler para este caso foram obtidos no exemplo 1 da seção $4.1 .7 \operatorname{como}(\alpha, \beta, \gamma)=(\pi, \pi / 2,0)$.

Portanto para descrever os estados $4 f$ no referencial do fóton, podemos utilizar a matriz de Wigner $\mathcal{M}_{m m^{\prime}}^{(3)}(\pi, \pi / 2,0)$, a qual foi calculada no exemplo 2 na equação (4.161). Se o campo é aplicado perpendicularmente à direção $x_{\phi}$ (isto é, na direção $y_{\phi}$ ), uma outra matriz similar deve ser calculada. Tanto na geometria Faraday quanto na geometria Voigt, o estado $5 d$ é descrito no referencial cujo eixo $z$ é orientado ao longo da direção cristalina [001]. Para descrever esse estado no referencial do fóton $\left(z_{\phi} \|\right.$ [111]), utiliza-se a matriz de Wigner de ordem 5 com os ângulos de Euler obtidos também no exemplo 1 da seção $4.1 .7 \operatorname{como}(\alpha, \beta, \gamma)=\left(\pi / 4, \tan ^{-1} \sqrt{2}, 0\right)$.

Uma vez determinadas as matrizes de Wigner adequadas, foram calculados os espectros em campo alto, os quais estão apresentados na figura 4.15. Observamos o surgimento das linhas dicróicas para $\sigma^{-}$e $\sigma^{+}$em ambas as geometrias Faraday e Voigt e o deslocamento para o vermelho devido à interação de exchange $d-f$, por um valor de $J_{d f} S=0.15 \mathrm{eV}$ em relação às posições em campo nulo.

Na geometria Faraday, os espectros de absorção em campos magnéticos não-nulos são diferentes para $\sigma^{-}$e $\sigma^{+}$e fornecem informação sobre a magnetização do material na direção de propagação da luz, que é também a direção do campo. Essa diferença é chamada dicroísmo circular magnético (DCM). Na geometria Voigt, o vetor de polarização pode ser orientado \| ou $\perp$ ao campo magnético externo, o qual por sua vez encontra-se orientado perpendicularmente à direção de propagação da luz. Assim, medidas na geometria Voigt trazem informação a respeito da anisotropia da distribuição de carga no plano perpendicular à direção de incidência. A diferença entre a absorção nas pola- 
a)

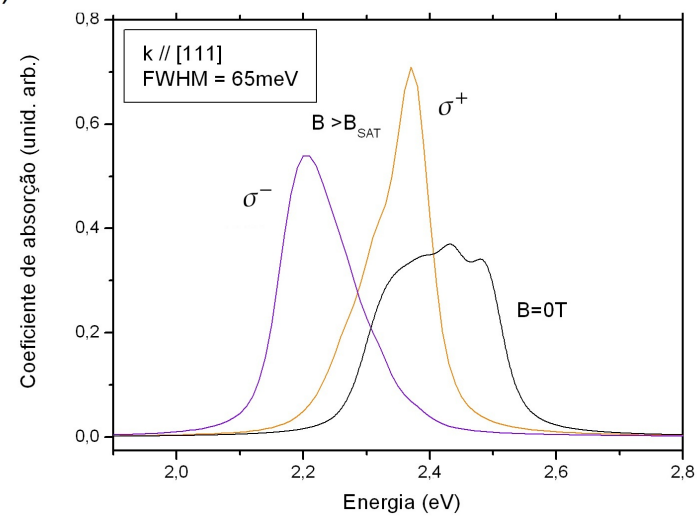

b)

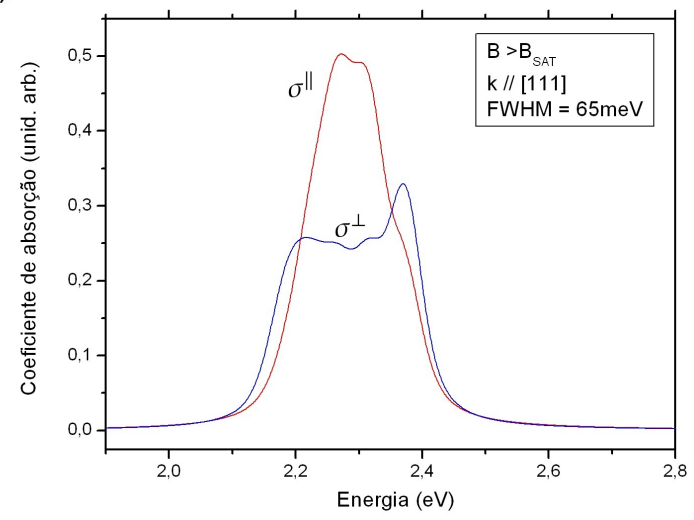

Figura 4.15: Espectros de absorção do EuTe na fase FM a) na geometria Faraday e b) na geometria Voigt.

rizações $\|$ e $\perp$ é chamada de dicroísmo linear magnético (DLM). Os espectros de DCM e DLM podem ser calculados com as definições apresentadas nas equações (4.190) e (4.191), onde $\alpha^{+}$e $\alpha^{-}$representam os coeficientes de absorção para LHCP e RHCP, respectivamente, e $\alpha^{\|}$e $\alpha^{\perp}$ são os coeficientes de absorção nas duas configurações da geometria Voigt.

$$
\begin{gathered}
D C M=\frac{\alpha^{+}-\alpha^{-}}{\alpha^{+}+\alpha^{-}} \\
D L M=\frac{\alpha^{\|}-\alpha^{\perp}}{\alpha^{\|}+\alpha^{\perp}}
\end{gathered}
$$

Para analisar a evolução dos espectros com o campo magnético, é necessário calcular as matrizes de Wigner para cada valor de campo, pois a direção dos spins da rede é determinada pela direção e magnitude do campo aplicado. A seguir serão apresentados os espectros de DCM e DLM para alguns valores de campo.

\section{Dicroísmo circular magnético e dicroísmo linear magnético no EuTe}

Em $B=0 \mathrm{~T}$, o sistema está na fase AFMII. A presença de um campo magnético externo cria uma força que tende a alinhar os spins em sua direção. Nesse processo, o primeiro efeito do campo é o de causar a transição spin-flop. Para $B=B_{s f}$ os spins da rede realinham-se 
abruptamente para um único domínio T cujo eixo AFMé orientado perpendicularmente ao campo magnético. A partir daí, o efeito do campo é reduzir o ângulo formado entre os vetores das magnetizações das duas sub-redes, que vai de $\theta=\pi$ radianos em $B=B_{s f}$, a $\theta=0 \mathrm{em} B=B_{S A T}$, quando o sistema atinge a fase FM.

Em campos um pouco acima ou um pouco abaixo da transição spin-flop, o DCM não varia de seu valor nulo, uma vez que o sistema permanece simétrico por inversão do sentido de rotação da luz circularmente polarizada. Entretanto o DLM pode variar bastante, uma vez que a anisotropia da distribuição de carga no plano de incidência pode mudar radicalmente com o realinhamento dos spins da rede. Por isso o DLM é uma técnica sensível para medidas do ordenamento dos spins no EuTe.

\section{Dicroismo circular magnético}

Em campo zero, como esperado para uma rede AFM, nenhum DCM é observado. Em um campo magnético de magnitude acima de $B_{S A T}$, o ordenamento FM dos spins da rede torna o material fortemente dicróico, como podemos observar na figura 4.16, que apresenta o espectro do DCM para o EuTe na fase FM.

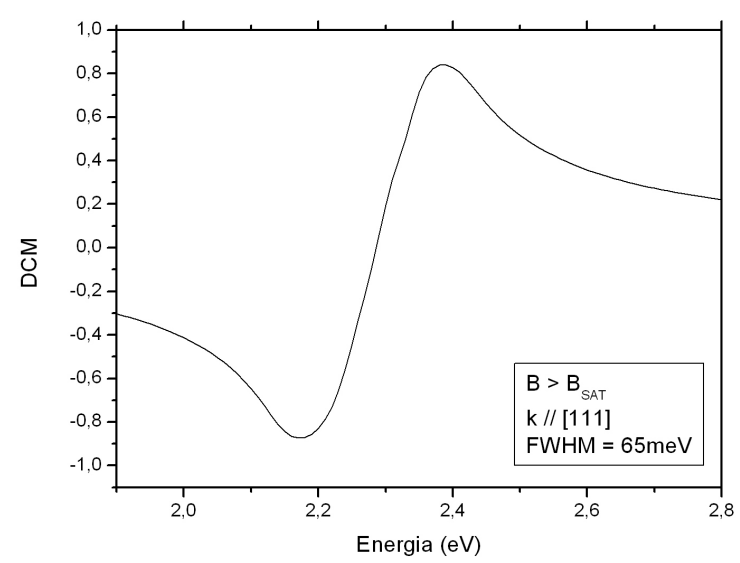

Figura 4.16: Espectro de DCM para EuTe na fase FM. 


\section{Dicroísmo linear magnético}

Em $B=0$ T devemos calcular o espectro de absorção fazendo a média sobre os espectros de todos os domínios magnéticos. Ao contrário do espectro de DCM, em campo nulo o sistema apresenta um espectro do DLM não nulo, como podemos ver na figura 4.17 a). Na fase FM, um forte dicroísmo é observado no espectro, cerca de 10 vezes maior do que em campo nulo, como vemos na figura $4.17 \mathrm{~b}$ ).
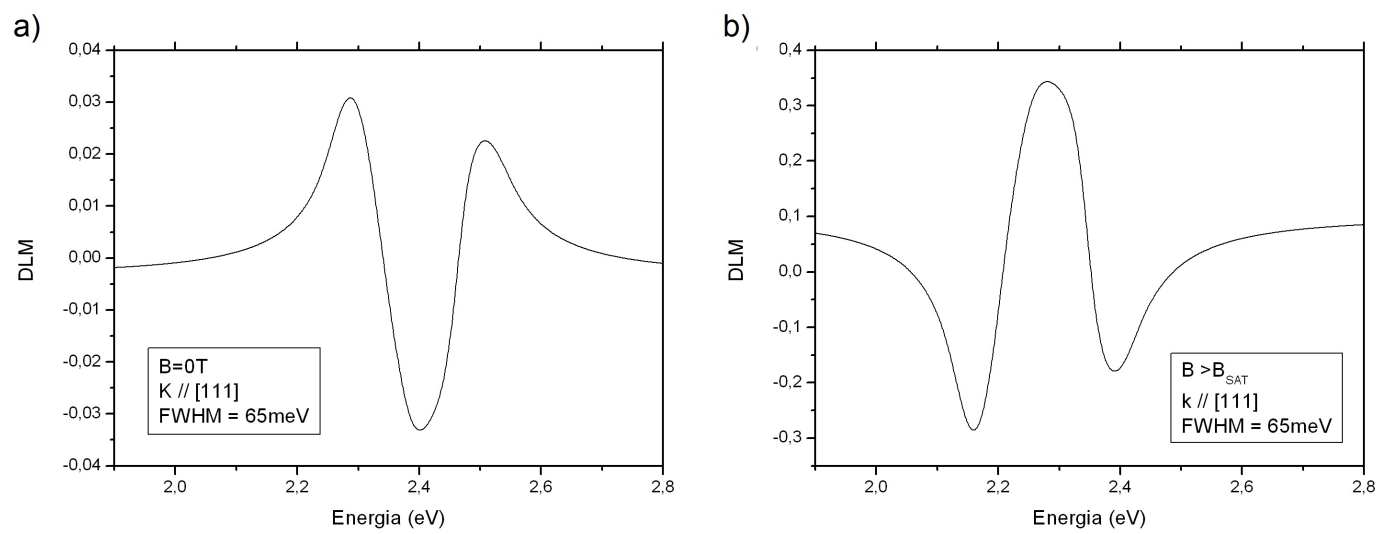

Figura 4.17: Espectro de DLM para EuTe com $\boldsymbol{k} \|$ [111] nas fases a) AFM e b) FM.

Em campos intermediários é necessário levar em consideração o comportamento dos spins da rede com a variação do campo. Antes da transição spin-flop cada um dos 12 domínios magnéticos está orientado em relação ao campo de forma diferente. A orientação de cada domínio em função do campo pode ser calculada por minimização do hamiltoniano (2.182). Ao calcularmos o espectro do DLM fazendo a média sobre todos os domínios, temos um espectro médio, o qual apresenta um aumento abrupto de intensidade no sinal do DLM na transição spin-flop. A figura 4.18 a) mostra os espectros de DLM em campo nulo, e para dois valores de campo: logo abaixo e logo acima de $B_{s f}$, evidenciando a mudança repentina da ordem magnética causada pelo campo externo. Para um particular domínio isolado, o espectro de DLM da figura 4.18 b) mostra uma inversão total do sinal após a transição spin-flop. Entretanto isso não é sempre verificado, pois em um domínio onde os spins já encontram-se orientados perpendicularmente ao campo antes da transição spin-flop, não seria observada diferença alguma no espectro 
a)

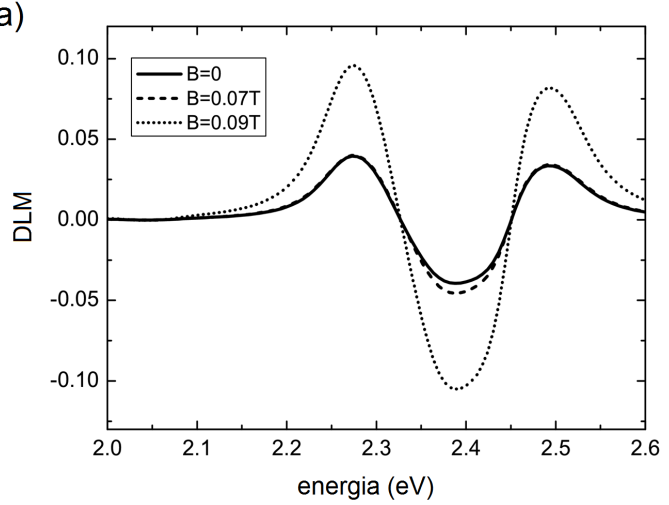

b)

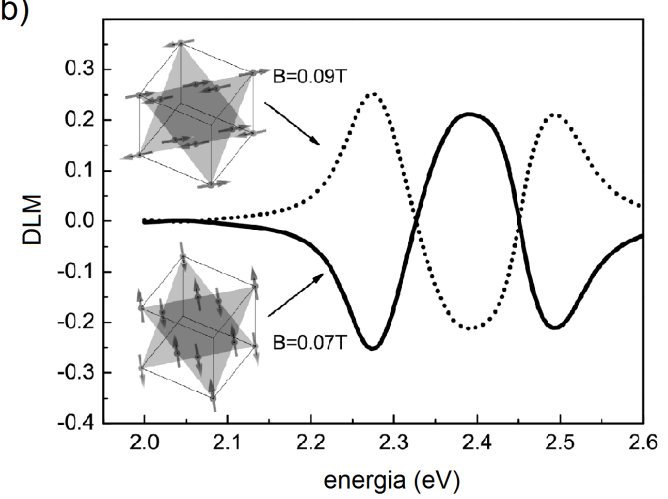

Figura 4.18: Espectros de DLM a) do EuTe em campos nulo, abaixo e acima da transição spin-flop e b) de um único domínio magnético do EuTe, ilustrado nos painéis internos.

de DLM na transição, pois o arranjo dos spins permaneceria o mesmo antes e após a transição.

Os espectros de DLM compostos pela média sobre todos os domínios foram calculados em função do campo magnético. O resultado pode ser observado na figura 4.19, onde podemos notar que o DLM anula-se para uma particular magnitude de campo, $B \sim 5.2 \mathrm{~T}$, um efeito de potencial interesse para o controle da ordem magnética do EuTe através da luz.

Os resultados do modelo para o DLM no EuTe foram publicados nas referências [28] e [81].

O espectro de DLM pode fornecer informações sobre o sistema que não são obtidas através do espectro de DCM em campo nulo, como por exemplo a predominância de algum domínio magnético no cristal. De forma geral é possível concluir que a partir dos espectros de DCM e DLM é possível obter informações acerca do estado magnético do sistema, sendo portanto uma ferramenta de potencial utilidade em aplicações magnetoópticas. A possibilidade de controlar o DLM com a aplicação de um campo magnético também é um efeito notável, que poderia ser aproveitado em dispositivos magnetoópticos. 


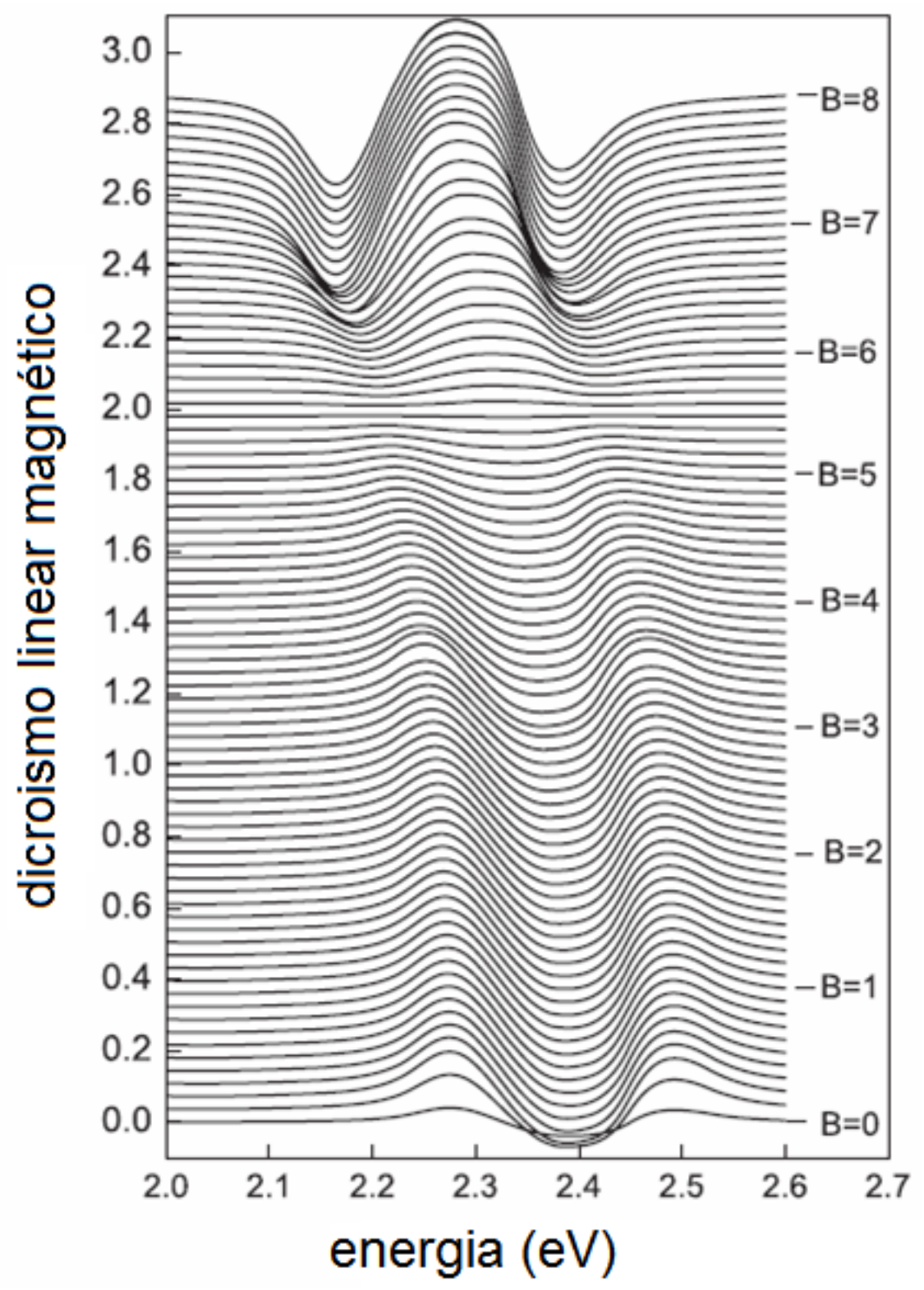

Figura 4.19: Espectro de DLM do EuTe em função do campo magnético aplicado. 


\subsection{Espectros de absorção no EuTe e EuSe}

Para obter os espectros de absorção das amostras epitaxiais de EuTe e EuSe descritas na seção 3.1, foi utilizado o arranjo experimental descrito na seção 3.2.1.

De acordo com o modelo da transição $4 f-5 d$ dos EuX apresentado na seção 4.1, um dos elétron que ocupa o nível $4 f$ pode absorver um fóton de energia $E_{G}$ ou maior, e saltar para a banda de condução. Se a energia dos fótons incidentes é menor do que $E_{G}$, o material não absorve luz, e portanto é transparente. Se a energia dos fótons for igual ou maior que $E_{G}$, o material pode absorvê-los, sendo portanto opaco para radiação com essas energia.

Em campo zero, os espectros de absorção do EuTe e EuSe da figura 4.20 reproduzem o comportamento esperado, apresentando um aumento abrupto da absorção a partir de certa energia de excitação.

Quando um campo magnético externo é aplicado sobre o sistema, duas linhas estreitas emergem nas proximidades do gap. As amplitude das linhas aumentam com o campo e a posição dos centros desloca-se para energias mais baixas até uma magnitude de campo magnético de $B=8.3 \mathrm{~T}$, a partir da qual não há mais deslocamento. O enorme deslocamento para o vermelho do máximo das linhas de absorção com o aumento do campo só pode ser entendido por um ganho de energia devido à interação de exchange entre o elétron fotoexcitado, que está num estado não-localizado, e os elétrons bem localizados dos orbitais $4 f$ dos sítios de európio da rede.

Os espectros de absorção de luz circularmente polarizada do EuTe em função do campo magnético aplicado na geometria Faraday são apresentados na figura 4.21 - que foi publicada na referência [24] - onde é possível notar a emergência das linhas dicróicas $\sigma^{-}$e $\sigma^{+}$.

Como vimos na seção 4.1.6, o elétron fotoexcitado reduz sua energia quando o campo magnético alinha os spins da rede, o que é refletido no deslocamento das linhas de absorção. Na figura 4.22 é possível observar um ajuste de (4.115) à posição das linhas $\sigma^{-}$e $\sigma^{+}$, obtidas experimentalmente em função do campo magnético para o EuTe. A partir desse ajuste foi possível determinar as constantes de exchange $J_{d f}=0.13 \mathrm{eV}$ para 


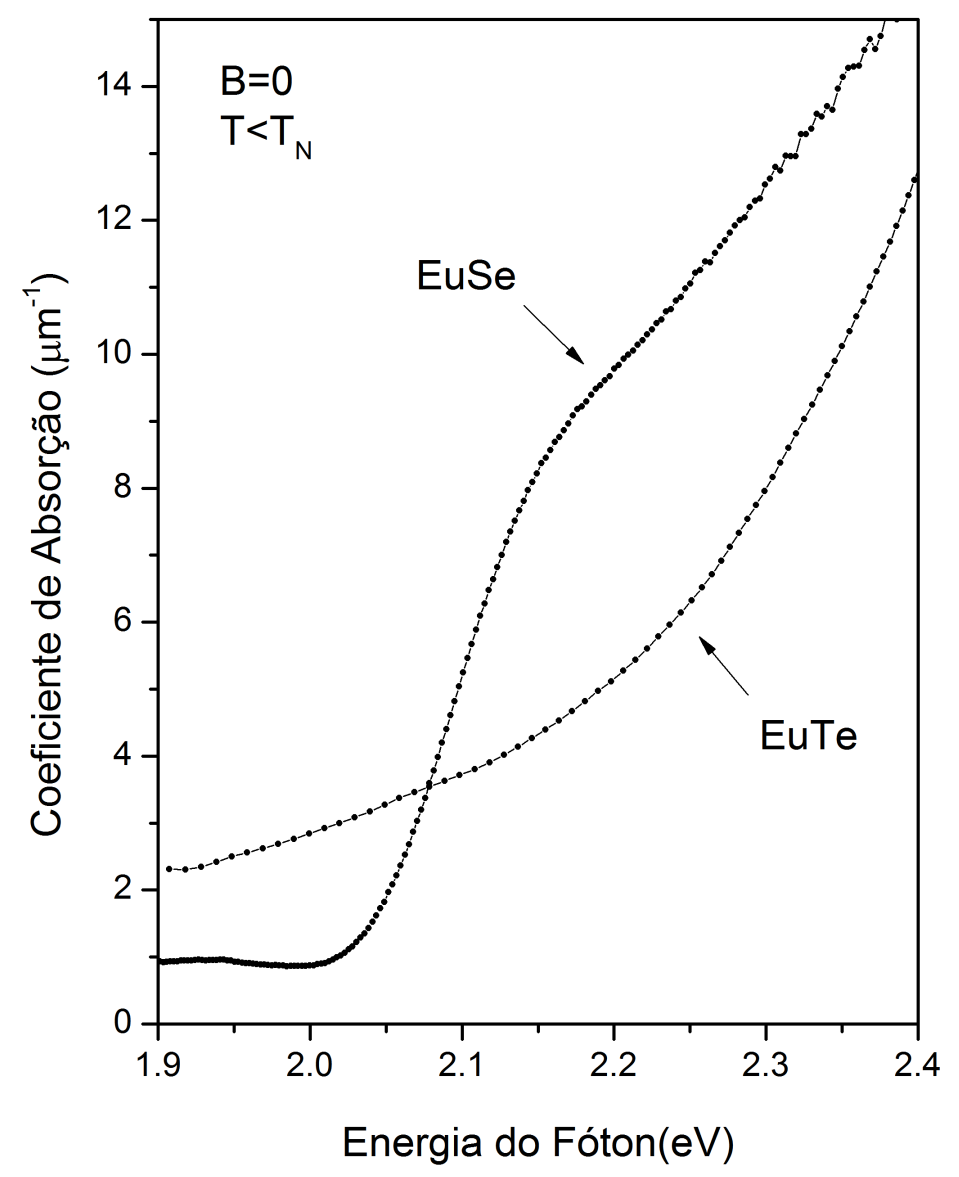

Figura 4.20: Espectros de absorção do EuSe e EuTe em campo zero.

a linha $\sigma^{-}$e $J_{d f}=0.09 \mathrm{eV}$ para $\sigma^{+}$.

O espectro de dicroísmo circular magnético do EuTe foi medido em função do campo magnético aplicado e comparado com os espectros teóricos. A figura 4.23, publicada na referência [26], mostra essa comparação, de onde fica evidente que o modelo teórico descreve muito bem os resultados experimentais.

No EuTe a constante de exchange AFM, $J_{2}$, é predominante, de forma que a fase AFMII é observada em todas as temperaturas entre zero e $T_{N}$. No EuSe a constante de exchange FM de primeiros $J_{1}$, tem magnitude muito próxima da constante de exchange AFM de segundos vizinhos $J_{2}$. Por isso um balanço delicado entre arranjos FM e AFM ocorre no EuSe, o qual é muito sensível a variações na temperatura, no campo magnético e na pressão. O resultado disso é o rico diagrama de fases $B-T$ do EuSe, apresentado 

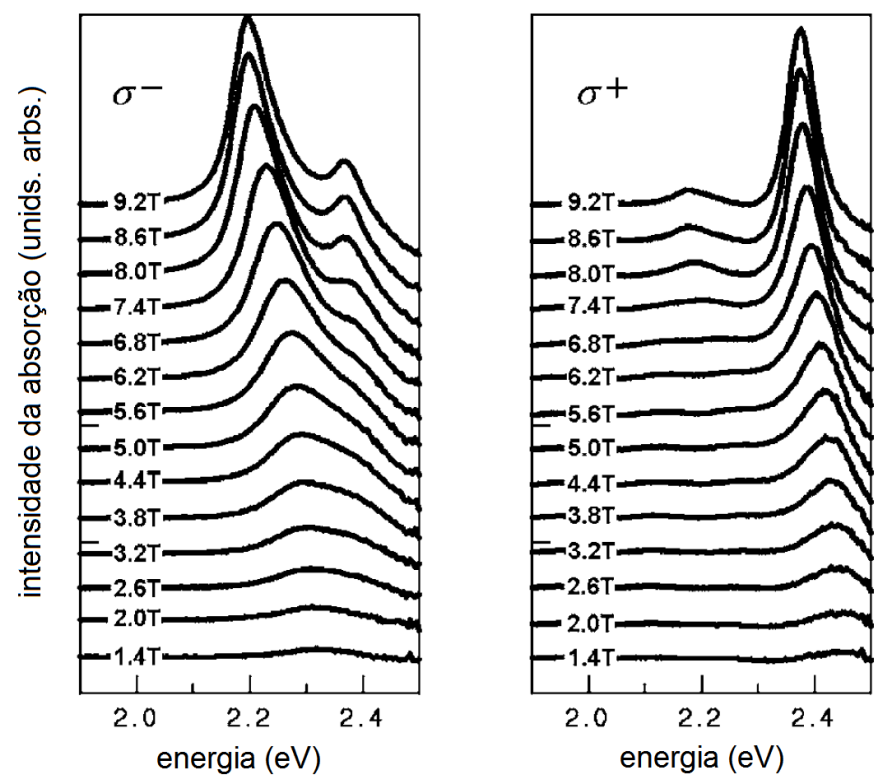

Figura 4.21: Espectros de absorção do EuTe na geometria Faraday em função do campo magnético. O espectro em campo zero foi subtraído de todas as curvas e as curvas são apresentadas com um deslocamento vertical para maior clareza.

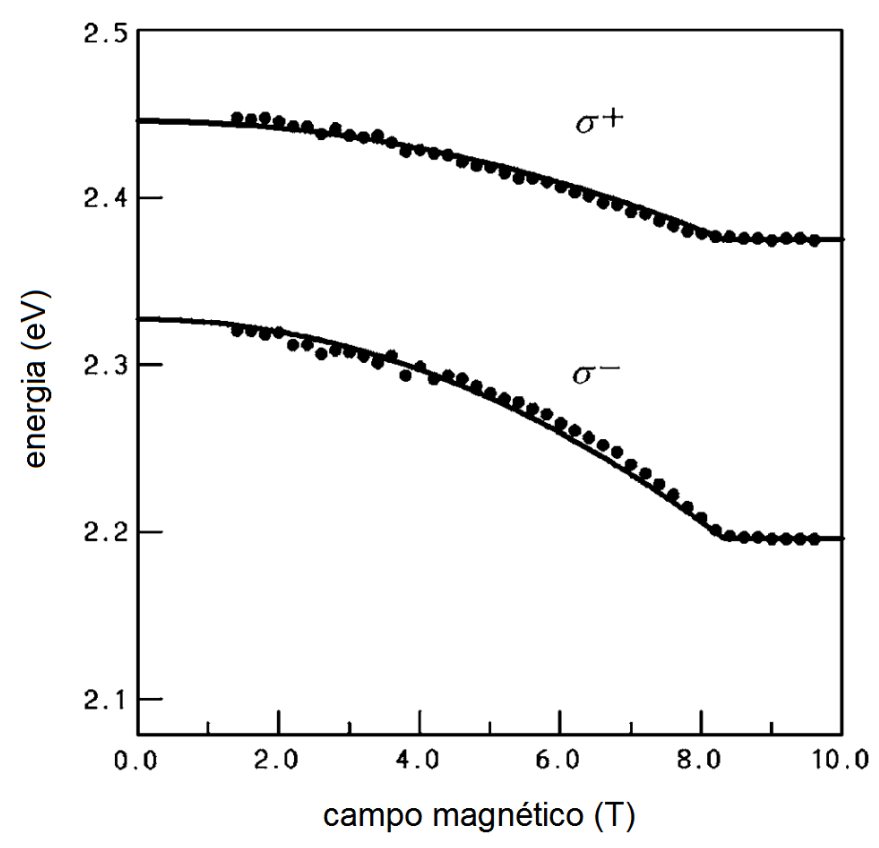

Figura 4.22: Posição das linhas $\sigma^{-}$e $\sigma^{+}$em função de $B$. As linhas representam o ajuste da equação (4.115) aos pontos experimentais. 

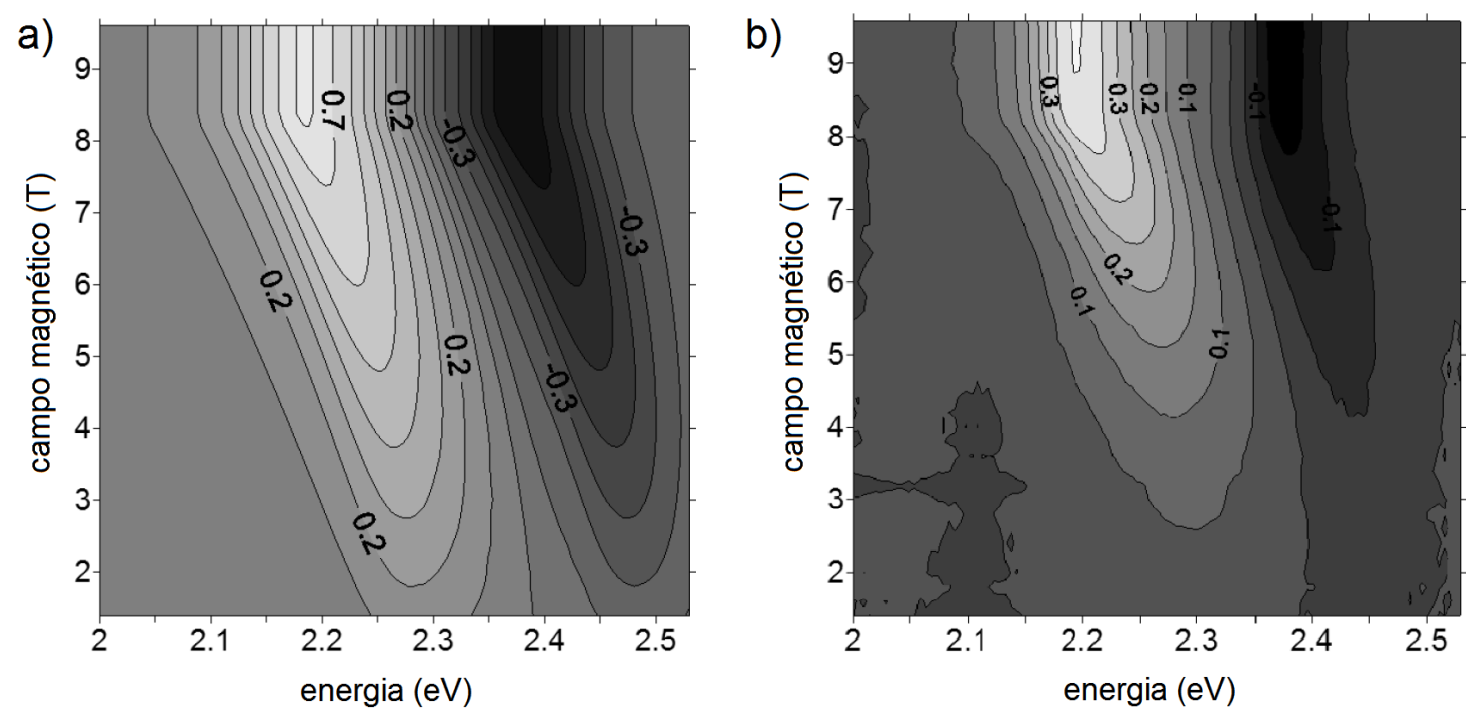

Figura 4.23: Espectros do DCM do EuTe em função do campo magnético a) teóricos e b) experimentais.

na figura 4.24, onde podemos identificar cinco arranjos magnéticos distintos.

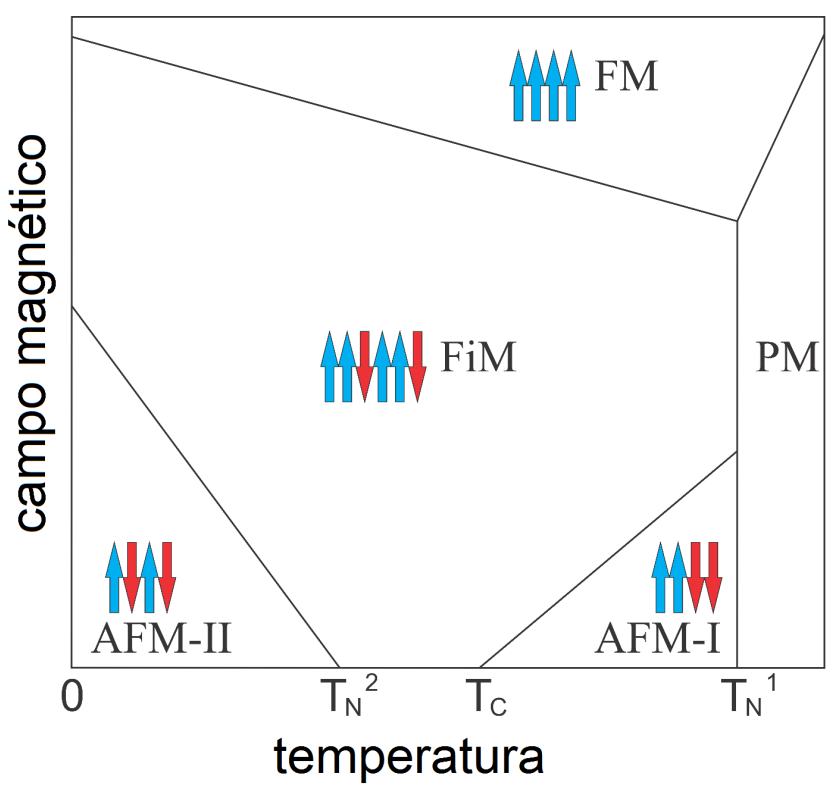

Figura 4.24: Diagrama $B-T$ das fases magnéticas do EuSe

Em $T=0 \mathrm{~K}$, o sistema encontra-se na fase AFMII, idêntica à do EuTe. Nessa temperatura a aplicação de um campo magnético perpendicular aos planos epitaxiais, nos quais estão contidos os spins da rede, gradualmente origina uma fase FiM, caracteri- 
zada por um padrão $\uparrow \uparrow \downarrow$ dos spins dos planos FM. Em um valor de campo interno $B_{S A T}^{1}$ o alinhamento FiM completo é atingido e a magnetização total da rede vale $M_{S} / 3$. O subsequente aumento do campo, causa um alinhamento gradual dos spins da rede para a direção do campo, de forma que em uma magnitude $B_{S A T}$ do campo interno, todos os spins da rede encontram-se alinhados e o sistema está na fase FM de magnetização total $M_{S}$.

Em temperaturas não-nulas, o ordenamento magnético do EuSe pode assumir diferentes formas correspondentes às diferentes fases magnéticas do diagrama 4.24. Acima de $T_{N}^{1}$ a fase AFMII deixa de existir, dando lugar ao arranjo FiM dos spins dos planos epitaxiais, o qual prevê uma magnetização não nula para o EuSe mesmo em campo zero. Essa fase se mantém até a temperatura $T_{C}$, onde a magnetização total anula-se novamente em virtude de um alinhamento AFMI da rede, o qual permanece até a temperatura de Néel $T_{N}=4.6$ (1) K [19], a partir da qual o sistema entra na fase paramagnética. Em cada uma dessas fases magnéticas observadas em campo magnético nulo, o efeito da aplicação de um campo magnético é diferente. Para temperaturas entre zero e $T_{N}^{1}$, a aplicação do campo magnético causa no sistema um comportamento análogo ao observado em $T=0 \mathrm{~K}$, onde o campo magnético induz a fase FiM em uma magnitude $B_{S A T}^{1}$ e a fase FM em uma magnitude $B_{S A T}$. Para temperaturas entre $T_{N}^{1}$ e $T_{C}$, o sistema encontra-se na fase FiM e a aplicação do campo magnético alinha os spins da rede para sua direção, fazendo com que a magnetização aumente gradualmente de $M_{S} / 3$ em $B=0 \mathrm{~T}$ para $M_{S}$ em $B=B_{S A T}$. Na região de temperaturas entre $T_{C}$ e $T_{N}$, em campo nulo, o ordenamento é AFMI. A aplicação do campo magnético causa uma transição para a fase FiM em uma magnitude de campo $B_{S A T}^{2}$ e posteriormente induz a fase FM em uma magnitude $B_{S A T}$.

Na figura 4.25, apresentamos os espectros de absorção do EuSe em função do campo magnético aplicado para uma temperatura de $T=4.40(1) \mathrm{K}$, um pouco abaixo da temperatura de Néel $T_{N}=4.6 \mathrm{~K}$. Nessa temperatura, em campo nulo, o EuSe encontrase na fase AFMI. Entretanto, para o primeiro valor de campo aplicado, $B=0.2 \mathrm{~T}$, de acordo com a referência [82] é esperado que o EuSe esteja na fase FiM, de forma que 
a transição $\mathrm{AFMI} \rightarrow \mathrm{FiM}$ não pode ser identificada nos espectros. É possível notar que com o aumento do campo magnético, as linhas dicróicas $\sigma^{-}$e $\sigma^{+}$emergem nos espectros e deslocam-se para o vermelho, apresentando uma saturação em B 2.6 T.
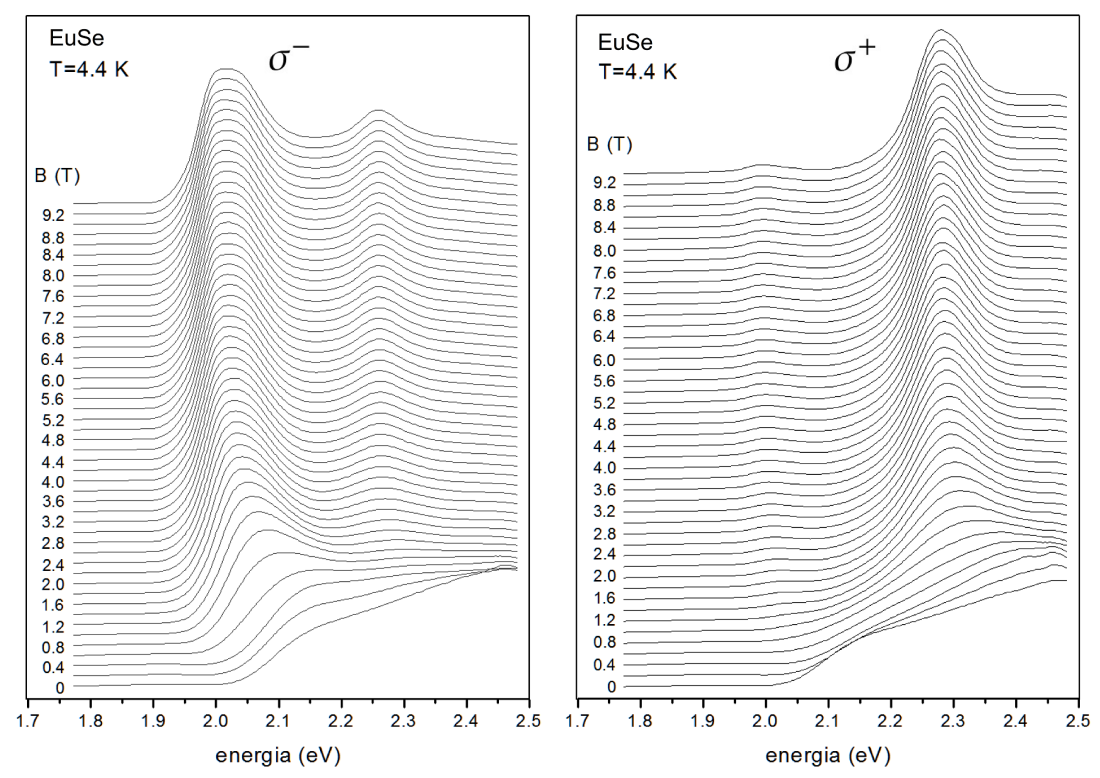

Figura 4.25: Espectros de absorção do EuSe na geometria Faraday em função do campo magnético. Um deslocamento vertical foi introduzido para maior clareza.

O deslocamento das linhas $\sigma^{-}$e $\sigma^{+}$, como no caso do EuTe é compreendido pela interação de exchange entre o elétron fotoexcitado e os íons $\mathrm{Eu}^{2+}$ da rede, a qual foi formulada na equação (4.106) como

$$
\mathcal{H}_{d f}=-\frac{2 J_{d f}}{\mathcal{N}} \sum_{i}^{\mathcal{N}} \boldsymbol{s}_{d} \cdot \boldsymbol{S}_{i}
$$

Como $\mathcal{H}_{d f}$ depende da orientação dos spins da rede, no EuSe, o deslocamento das linhas dependerá da fase magnética em que se encontra o material. Dessa forma, o deslocamento das linhas em função do campo para temperaturas fixas pode ser utilizado para detectar as fases magnéticas do EuSe, o que consiste em uma técnica óptica para obtenção de características magnéticas do sistema, a qual será explorada na seção seguinte. 


\subsubsection{Identificação das fases magnéticas no EuSe pelo deslocamento da linhas de absorção}

Em $B=0$ T o espectro de absorção é igual para as duas polarizações da luz, apresentando uma curva que divide o espectro em duas regiões, uma transparente e uma opaca, como podemos ver na figura 4.25. Na presença do campo magnético, o elétron na banda $5 d$ realinha o spin dos sítios dentro do volume de interação, causando o deslocamento para o vermelho das linhas de absorção. Essa redução na energia é proporcional à magnetização e depende da fase magnética em que encontra-se o material.

Para identificar a fase magnética do EuSe através do deslocamento das linhas de absorção, foram obtidos espectros de absorção em função do campo para duas temperaturas fixas distintas: $T=1.60(1) \mathrm{K}$, a qual está abaixo de $T_{N}^{1}=1.8 \mathrm{~K}$ dada pela referência [58] e T=4.50 (1) K, que está abaixo da bem conhecida temperatura de Néel do EuSe $T_{N}=4.6 \mathrm{~K}[19]$. Para obtermos separadamente as linhas $\sigma^{-}$e $\sigma^{+}$os espectros foram medidos com controle de polarização. Com base na evolução da posição da linha $\sigma^{-}$ em função do campo, pretendemos detectar a fase magnética em que se encontra o EuSe e comparar com a previsão do diagrama da figura 4.24.

Na temperatura $T=4.5 \mathrm{~K}$ foram obtidos espectros de absorção em função do campo para campos de zero a $9.5 \mathrm{~T}$, com passo de $0.1 \mathrm{~T}$. O resultado pode ser observado na figura 4.26 a), onde os espectros são apresentados com curvas de nível, segundo a escala de cores à direita do gráfico. Nota-se o máximo que surge e desloca-se para energias mais baixas na medida em que aumenta-se o campo magnético, até saturação FM, que ocorre em 2.2 T. Uma varredura com passo mais fino de campo revelou-se necessária para compreender a sinuosidade na região do espectro contida no retângulo branco da figura 4.26 a), o que foi feito para campos de até $1 \mathrm{~T}$ com passo de $0.02 \mathrm{~T}$, como pode ser visto na figura $4.26 \mathrm{~b}$ ). No intervalo de $B=0$ a $1 \mathrm{~T}$, a linha $\sigma^{-}$ainda não emergiu o suficiente para que se possa definir um centro e por isso o limiar da absorção foi utilizado para determinar a energia do gap (linha tracejada na fig. 4.26 b)). Além disso os espectros de transmissão também foram analisados segundo o método de [83], levando a resultado semelhante qualitativamente (pontos na fig. 4.26 b)), mas 
levemente deslocado em energia.
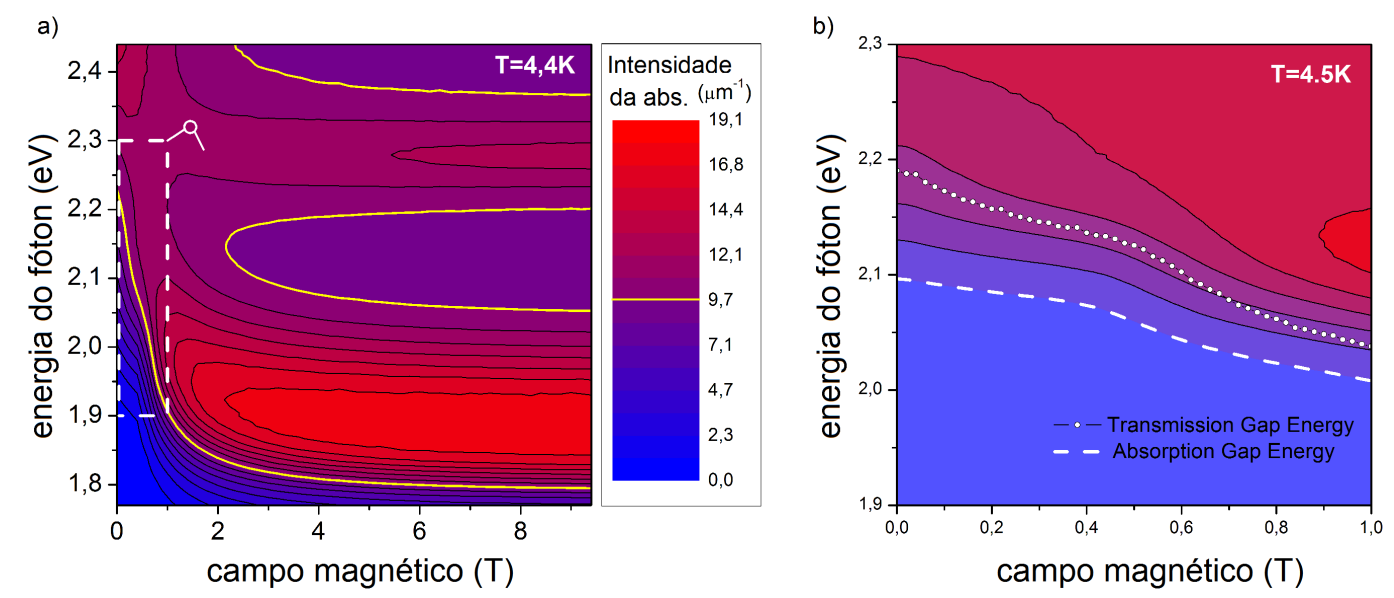

Figura 4.26: Contorno do espectro de absorção em função do campo. A região em vermelho corresponde à linha $\sigma^{-}$. A linha amarela é a largura a meia altura da $\sigma^{-}$. A região demarcada com o tracejado apresenta uma sinuosidade que foi examinada com maior resolução, resultando no gráfico b), onde observa-se que a saturação FiM ocorre em $B \simeq 0.64 \mathrm{~T}$.

Na figura $4.26 \mathrm{~b}$ ) observa-se que em $0<B<0.4$ T existe um patamar, o qual é associado à fase FiM. Em $B \simeq 0.5$ T nota-se uma mudança na derivada da curva tracejada, indicando que a saturação FiM foi atingida e que o campo magnético começa a alinhar os 2/3 dos spins restantes para a sua direção. A partir desse ponto, o deslocamento do gap de absorção com o campo magnético continua suavemente até que seja atingida a saturação FM (fig. 4.26 a)). A transição AFMI $\rightarrow$ FiM, prevista no diagrama $B-T$ do EuSe, não é visível nesse experimento, pois de acordo com [82], o campo de saturação dessa transição é de 0.02 T, o que corresponde ao menor passo de campo utilizado nos experimentos.

Em $T=1.6 \mathrm{~K}$ e $B=0 \mathrm{~T}$, o sistema encontra-se na fase AFMII e o aumento do campo magnético leva o sistema para a fase FiM e em seguida para a fase FM. O espectro de absorção função do campo nessa temperatura (figura 4.27 a)) permite identificar um patamar na energia do gap, que se estende de $B=0 \mathrm{~T}$ até $B \sim 0.18 \mathrm{~T}$, onde uma mudança na inclinação indica a transição para a fase FiM. Nesse ponto a magnetização na direção do 
campo ainda é ínfima, mas na medida que aumenta-se o campo a magnetização cresce rapidamente até o surgimento de outro patamar, centrado em $B \sim 0.6 \mathrm{~T}$, que pode ser associado à fase FiM, confirmando a possibilidade de identificar-se a fase magnética do EuSe através dos espectros de absorção.
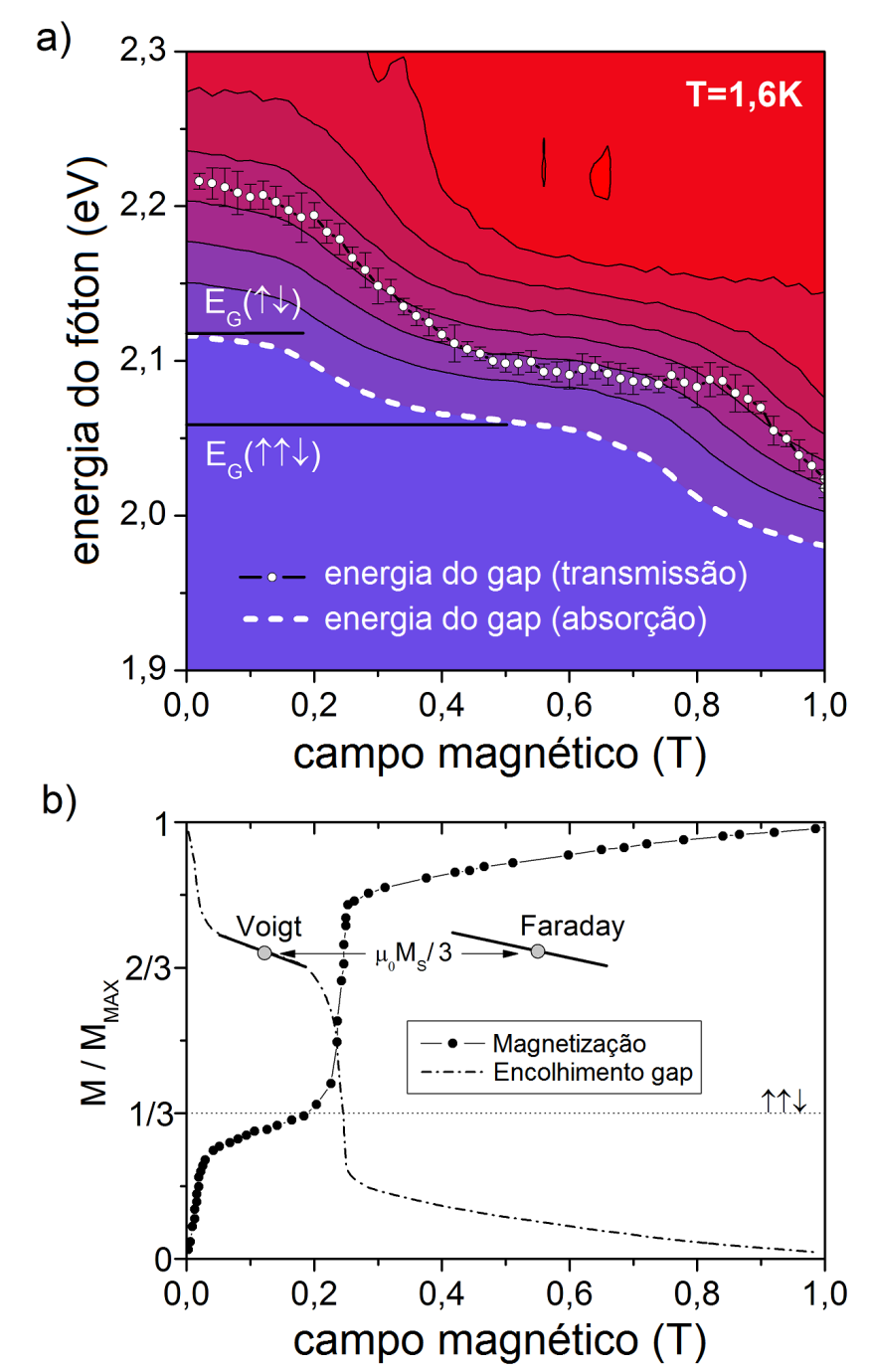

Figura 4.27: a) Contorno do espectro de absorção em função do campo. b) Estimativa do encolhimento do gap obtida através da magnetização da amostra medida na geometria Voigt e corrigida para a geometria Faraday.

Para reforçar este resultado, foi feita uma estimativa do encolhimento do gap em função do campo a partir de uma medida da magnetização da amostra na mesma temperatura. Nessa estimativa, a magnetização do EuSe, que foi medida na geometria 
Voigt deve ser corrigida para podermos comparar com nossas medidas realizadas na geometria Faraday, onde o campo de desmagnetização deve ser subtraído do campo aplicado para obtermos o campo magnético no interior do material (figura 4.27 b)). Por comparação, concluímos que o patamar observado está na posição esperada e portanto pode ser relacionado com a saturação da fase FiM. Em $B \sim 0.7$ T é possível observar a mudança na inclinação da curva tracejada, indicando que $\frac{1}{3}$ dos spins estão alinhados ao campo e os outros $\frac{2}{3}$ começam a ser reorientados rumo à saturação FM, onde não há mais deslocamento do gap de absorção.

Em conclusão, pode-se afirmar que a partir do espectro de absorção do EuSe tomado na geometria Faraday, foi possível identificar diferenças na ordem magnética do sistema em duas temperaturas distintas através do encolhimento do gap óptico associado ao deslocamento da linha de absorção $\sigma^{-}$. Enquanto que a $T=4.5 \mathrm{~K}$ a linha desloca-se suavemente com o campo até a saturação FM, em $T=1.6 \mathrm{~K}$ identificou-se uma saturação intermediária a aproximadamente um terço do deslocamento total, evidenciando a transição da fase AFMII para a fase FiM, esperada nessa temperatura. Esses resultados foram publicados na referência [84]. 


\subsection{Espectros de Fotoluminescência do EuTe}

Os EuX são semicondutores intrinsecamente magnéticos com uma alta densidade de momento magnético, uma das maiores em todos os materiais. Ao mesmo tempo, os EuX apresentam uma janela de transparência na região visível do espectro eletromagnético. Por isso a indução de polarons magnéticos em $\mathrm{EuX}$, através de sinal externo, é de grande interesse aplicativo, por exemplo em moduladores magneto-ópticos. Entretanto a indução de polarons magnéticos em EuTe e EuSe ainda é uma questão em aberto. Nesta seção é apresentada a investigação da fotoluminescência em EuTe, visando detectar evidências da indução óptica de polarons magnéticos.

Utilizando o arranjo experimental descrito na seção 3.2.2, foi realizada uma série de medidas do espectro de fotoluminescência (PL) do EuTe. Abaixo da temperatura de Néel, com energia de excitação maior que a do gap de absorção, foram observadas as faixas estreitas $\mathrm{MX}_{1}$ e $\mathrm{MX}_{2}$ de energias $1.92 \mathrm{eV}$ e $1.87 \mathrm{eV}$, respectivamente, reportadas anteriormente [85], as quais foram atribuídas a estados eletrônicos internos ao gap, não acessíveis por absorção. Além disso foi observada a luminescência larga que ocorre em torno de $1.5 \mathrm{eV}$.

Ao diminuir-se a potência de excitação, foi observado que as linhas $\mathrm{MX}_{1}$ e $\mathrm{MX}_{2}$ perdem intensidade e desaparecem para potências abaixo de $0.1 \mathrm{~mW} / \mathrm{cm}^{2}$, dando lugar a um conjunto de linhas estreitas igualmente espaçadas, que combinam-se formando uma banda de emissão de largura total de $\sim 60 \mathrm{meV}$, a qual foi denominada banda $\mathrm{MX}_{0}$. Os espectros de PL no intervalo de energias de $\sim 1.8$ eV a $2 \mathrm{eV}$ são apresentados na figura 4.28 , ilustrando essa situação.

A aplicação de um campo magnético causa um deslocamento rígido dos máximos de intensidade, como podemos ver na figura 4.29. Como no caso do gap da absorção, esse deslocamento só pode ser compreendido como o ganho de energia do elétron fotoexcitado devido à interação de exchange com os spins da rede.

Nas curvas de PL da figura 4.29 é possível notar uma estrutura de máximos, que é diferente nas duas amostras, as quais possuem espessuras diferentes. Essa diferença é compreendida pelo fato de que a PL de camadas epitaxiais de EuTe crescidas em 


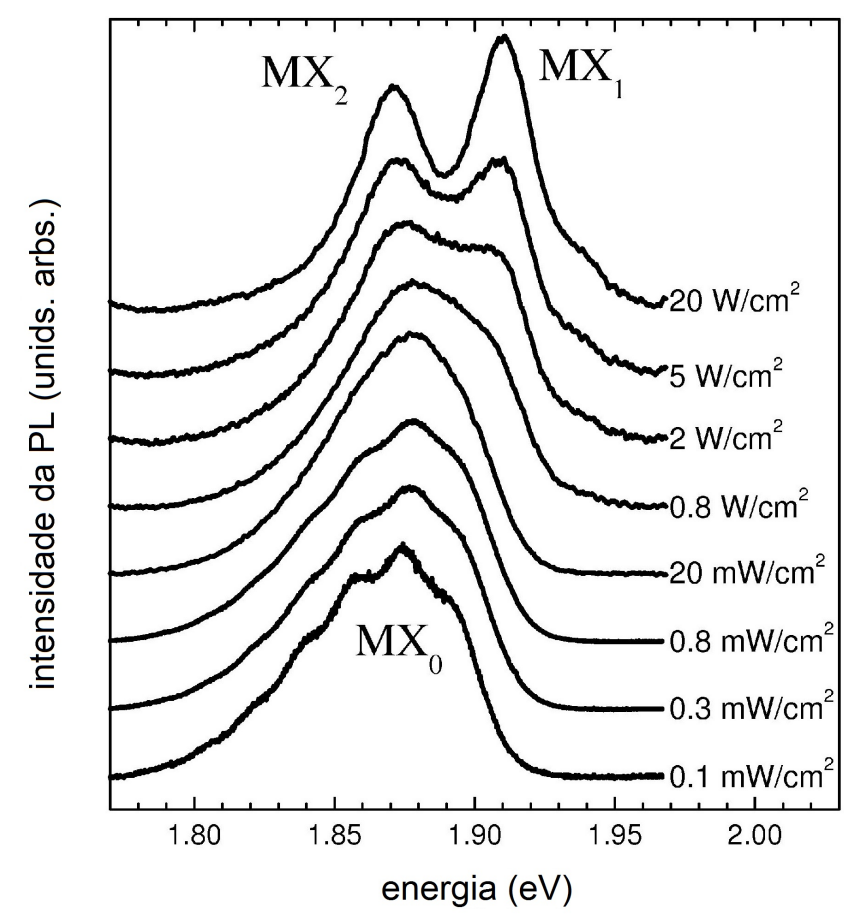

Figura 4.28: Espectro de fotoluminescência em função da potência de excitação.

substrato de $\mathrm{BaF}_{2}$, tem sua intensidade modulada por um efeito de interferência de Fabry-Perot entre a PL que emerge da amostra e a PL refletida pelo substrato [85], o que está ilustrado na figura 4.30. O período de oscilação da intensidade da PL pode ser estimado como se segue.

Em um filme de espessura $d$, a luz que é refletida pelo substrato e que emerge a uma distância $\delta<<d$ do ponto onde foi gerada a PL (ver figura 4.30), percorre uma distância $\sim 2 d$ no interior da camada, com uma velocidade $v=c / n$, onde $n$ é o índice de refração do material. Para que haja interferência construtiva, a PL detectada diretamente e a PL detectada após refletir no substrato devem estar em fase, o que implica que

$$
\frac{2 d}{\lambda_{m}}=N=1,2, \ldots
$$

onde $\lambda_{m}$ é a distância percorrida pela onda em um período no interior da camada. Mas

$$
\lambda_{m}=\frac{v}{f}=\frac{c / n}{f}=\frac{\lambda}{n}
$$

onde $\lambda$ é a distância percorrida em um período fora do material. Substituindo (4.194) 

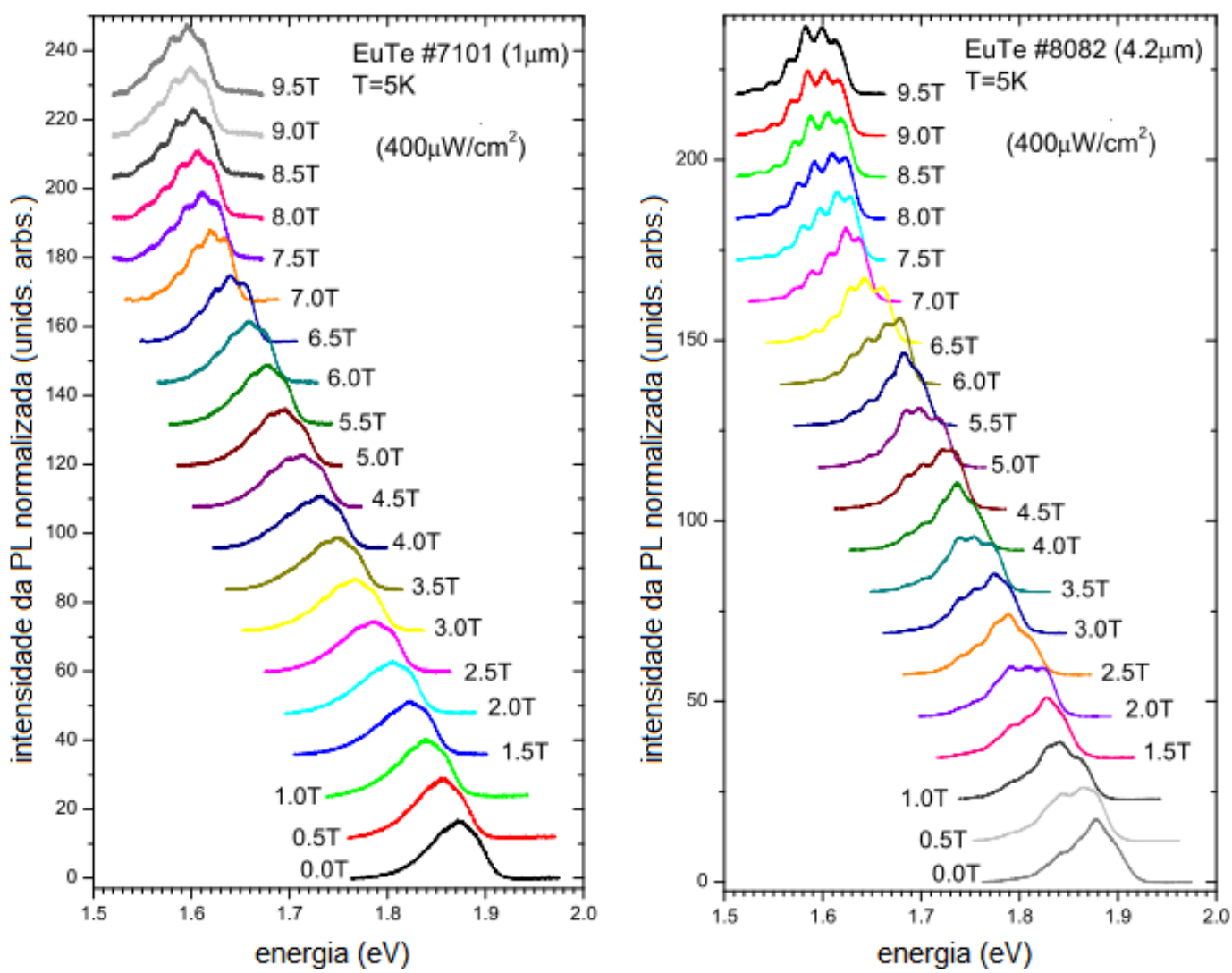

Figura 4.29: Espectros de PL normalizados em função do campo magnético para duas amostras diferentes. Para maior clareza, as curvas foram deslocadas verticalmente em função do campo magnético.

na equação (4.193) obtemos

$$
\frac{2 n d}{\lambda}=N=1,2, \ldots
$$

Para dois máximos consecutivos, $N_{1}$ correspondente a $\lambda_{1}$ e $N_{2}=N_{1}-1$ correspondente a $\lambda_{2}<\lambda_{1}$, temos

$$
\frac{2 n d}{\lambda_{1}}=N_{1} \quad \text { e } \frac{2 n d}{\lambda_{2}}=N_{1}-1
$$

O período de modulação da intensidade da PL, é dado pela diferença entre dois máximos consecutivos,

$$
\Delta\left(\frac{1}{\lambda}\right)=\frac{1}{\lambda_{1}}-\frac{1}{\lambda_{2}} \underbrace{=} \frac{1}{2 n d}
$$




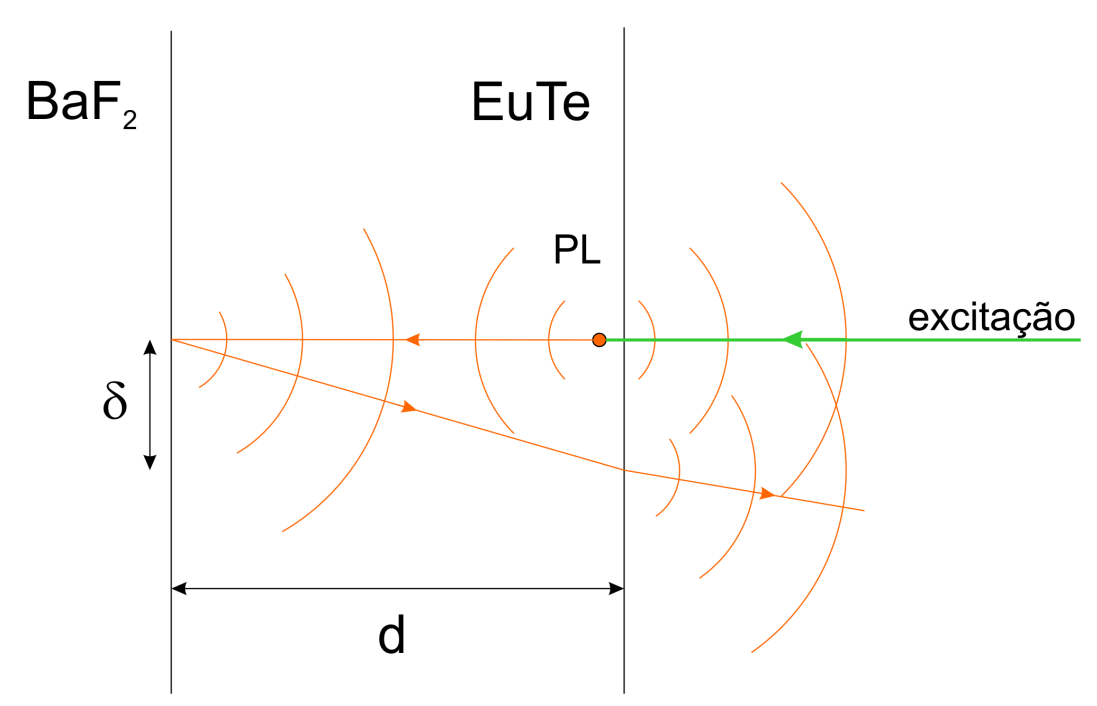

Figura 4.30: Interferência de Fabry-Perot da PL com a PL refletida no substrato.

o que pode ser escrito em termos da energia como

$$
\frac{1}{\lambda_{1}}-\frac{1}{\lambda_{2}}=\frac{1}{h c}\left(h v_{1}-h v_{2}\right)=\frac{\Delta(h v)}{h c}=\frac{1}{2 n d}
$$

portanto

$$
\Delta(h v)=\frac{h c}{2 n d}
$$

A energia da PL é de $\sim 1.88$ eV e o índice de refração do EuTe nessa energia é de $\sim 2.7$ [86], de forma que para a amostra de $4.2 \mu \mathrm{m}$, temos $\Delta(h v) \sim 55 \mathrm{meV}$, que é da ordem da largura da banda da PL ( 60 meV). Para a amostra da $1 \mu \mathrm{m}$, temos $\Delta(h v) \sim 230 \mathrm{meV}$, que é mais de três vezes maior que a largura da banda e portanto a modulação por interferência não pode ser associada à estrutura interna da PL. Na figura 4.29 vemos que a estrutura presente na amostra de $1 \mu \mathrm{m}$ também está presente na amostra mais espessa, entretanto pequenas variações aparecem devido ao efeito de interferência, que na amostra mais espessa manifesta-se visivelmente no espectro.

Para a amostra mais fina foi feita uma varredura da PL em função do campo com passo muito fino, de $0.015 \mathrm{~T}$, resultando no gráfico da figura 4.31. Notamos que o deslocamento das linhas com o campo é linear até uma magnitude de 7.1 T, onde uma mudança da inclinação nas retas $\mathrm{PL} \times \mathrm{B}$, indica a saturação magnética do sistema. No 


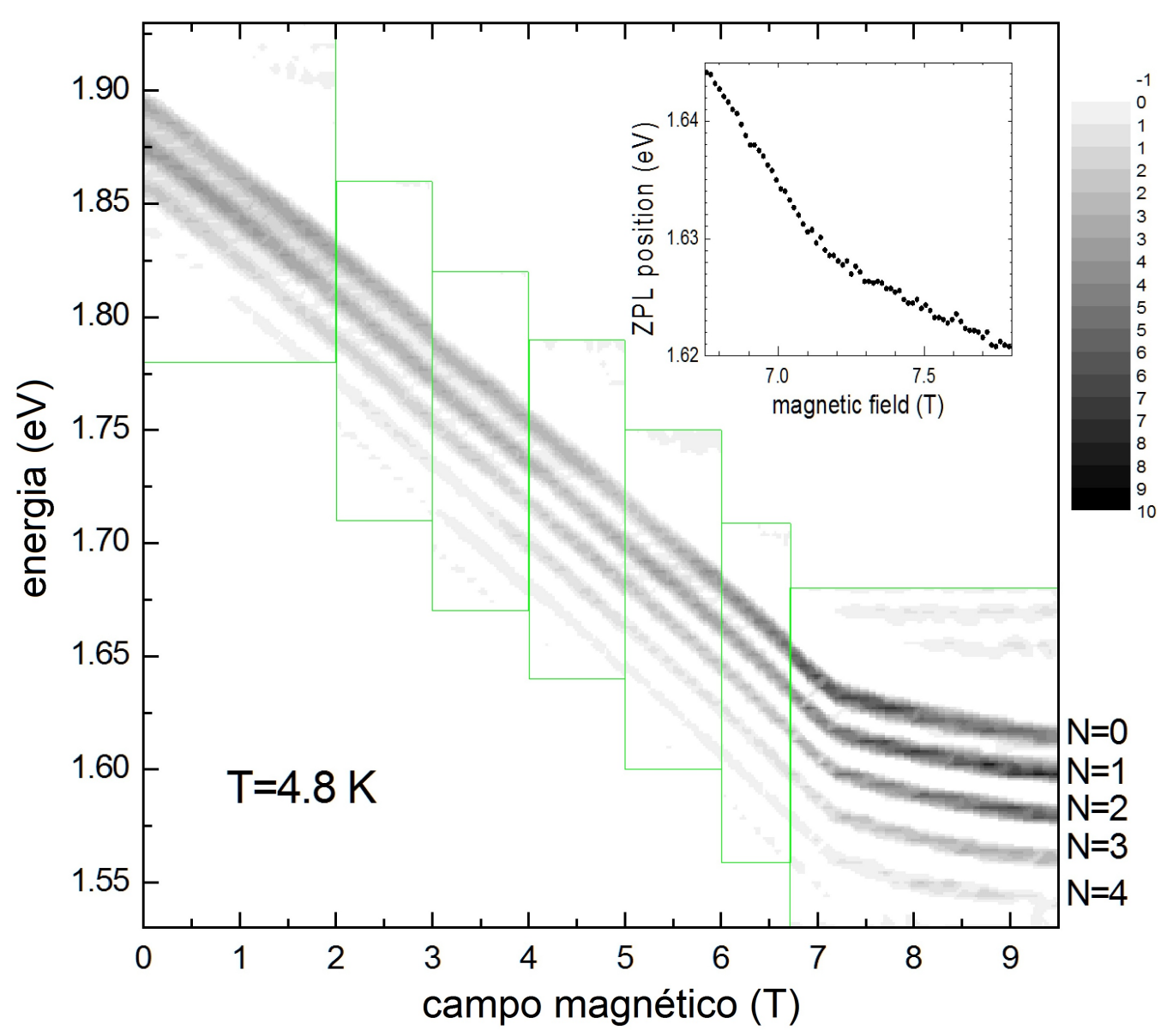

Figura 4.31: Deslocamento da banda $\mathrm{MX}_{0}$ em função do campo magnético aplicado. É possível resolver o conjunto de linhas igualmente espaçadas, as quais deslocam-se linearmente com o campo. A mudança da inclinação das retas, em detalhe no painel interno, identifica o campo de saturação em 7.1 T.

painel interno da figura 4.31 apresentamos a posição da ZPL em função do campo, obtida a partir das segundas derivadas dos espectros, apresentadas na figura 4.32, que permitem identificar com precisão que em $B=7.1 \mathrm{~T}$ a inclinação da reta muda drasticamente, evidenciando a saturação magnética dos spins da rede. Para campos acima de $7.1 \mathrm{~T}$, o elétron fotoexcitado continua a reduzir ligeiramente sua energia, o que será comentado mais adiante na seção 4.3.1.

Na figura 4.32 é possível identificar até seis linhas de luminescência igualmente espaçadas, as quais evidenciam um acoplamento da fotoluminescência com modos de 


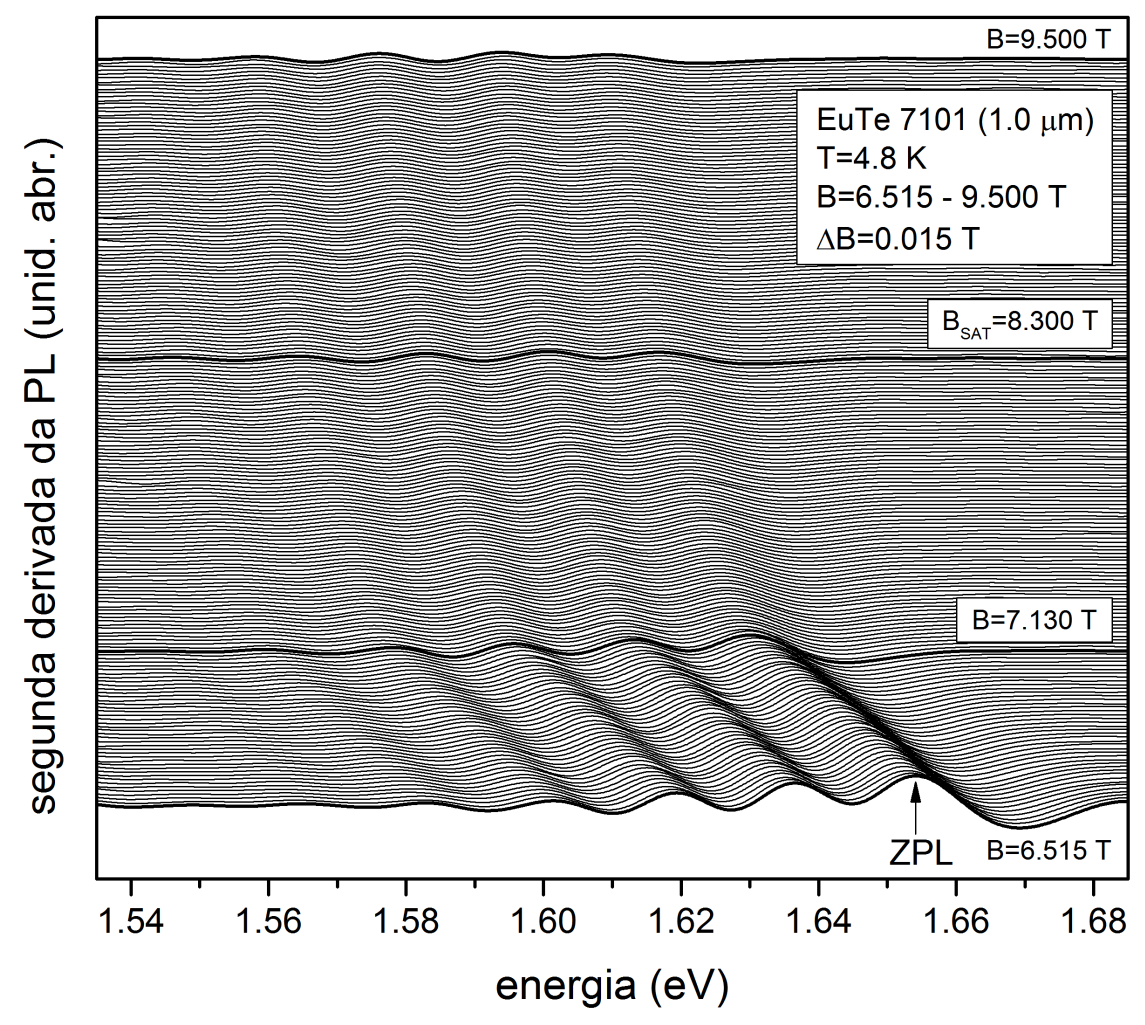

Figura 4.32: Segunda derivada da PL $M X_{0}$ em função do campo magnético aplicado.

vibração da rede. A transição eletrônica produz quanta vibracionais nos átomos da rede, os quais manifestam-se nos espectros de emissão. Esse processo está ilustrado na figura 4.33, onde vemos que a absorção ocorre com coordenadas fixas, de acordo com o Princípio de Franck-Condon [87], que estabelece que durante o rápido processo de absorção, a enorme massa do núcleo comparada à do elétron não tem tempo de mover-se para a nova posição de equilíbrio. Entretanto, antes da recombinação, o elétron fotoexcitado tem tempo para relaxar para um estado $X$ de mínima energia, antes de voltar para o estado fundamental, que agora é composto por modos normais de vibração. O espaçamento entre as linhas de $\hbar \omega=17.5 \mathrm{meV}$, corresponde exatamente à energia do fônon óptico longitudinal no EuTe [88], o que permite a identificação desses modos vibracionais.

A figura 4.34 mostra a emissão $\mathrm{MX}_{0}$ para amostras de 1, 1.5 e $4.2 \mu \mathrm{m}$ em um campo magnético $B=9.5 \mathrm{~T}$, onde podemos notar que a estrutura interna da banda $\mathrm{MX}_{0}$ tem resolução maior nas camadas mais espessas. Isso pode ser compreendido pelo 


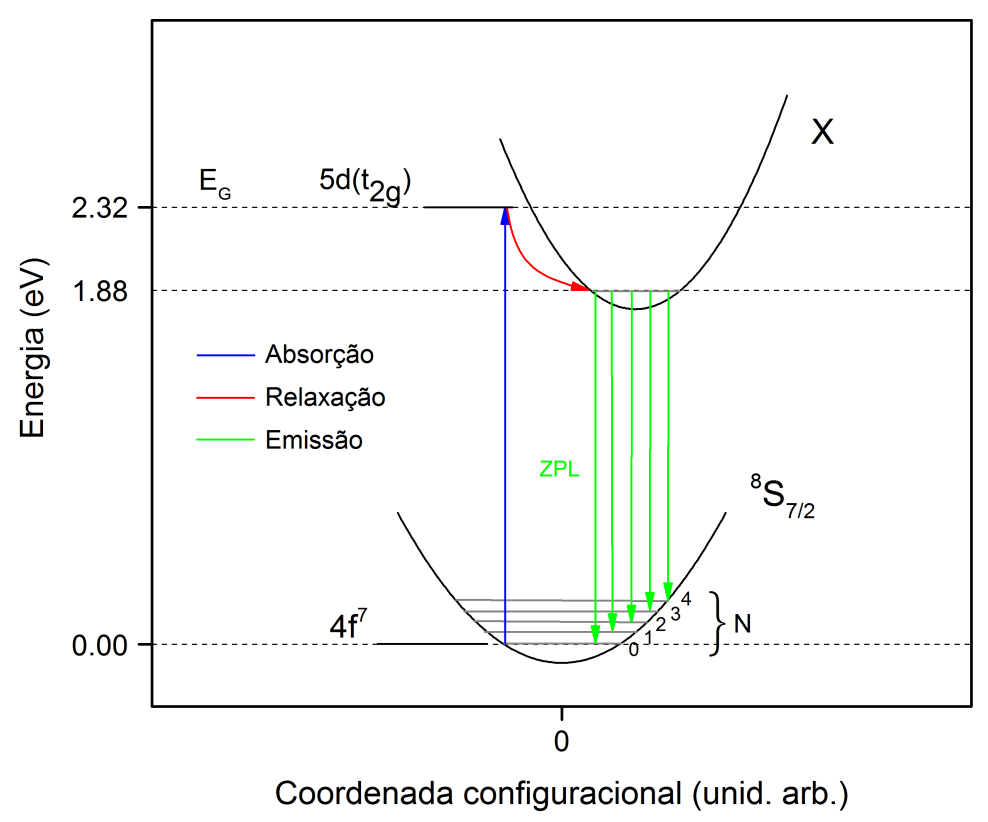

Figura 4.33: Precesso de geração da luminescência $M X_{0}$.

fato de que nas amostras mais espessas a emissão ocorre em regiões mais afastadas da interface $\mathrm{BaF}_{2} / \mathrm{EuTe}$, onde há uma grande concentração de defeitos estruturais em virtude da incompatibilidade de $6 \%$ entre os parâmetros de rede da camada e do substrato. Podemos notar também que o espaçamento entre as linhas é independente da espessura da camada, o que confirma que a estrutura na banda $\mathrm{MX}_{0}$ não está relacionada com interferência de Fabry-Perot.

A distribuição das intensidades das linhas associadas a cada modo normal de vibração, é bem descrita no modelo harmônico linear, que de acordo com as referências [89] e [90], prevê uma distribuição de Poisson de intensidades dadas por

$$
I=\frac{e^{-S} S^{N}}{N !}
$$

onde $\mathrm{N}=0,1, \ldots$, é o número de quanta de vibração gerados na transição e $S$ é fator de Huang-Rhys, que caracteriza a intensidade do acoplamento entre a transição eletrônica e os modos vibracionais.

$\mathrm{Na}$ amostra mais fina $(1.0 \mu \mathrm{m})$, onde a modulação da envoltória da banda $\mathrm{MX}_{0}$ é pouco afetada pela interferência de Fabry-Perot, a banda $\mathrm{MX}_{0}$ foi ajustada à distribuição de Poisson de intensidades da equação (4.200). A curva envoltória da banda $\mathrm{MX}_{0}$ foi 


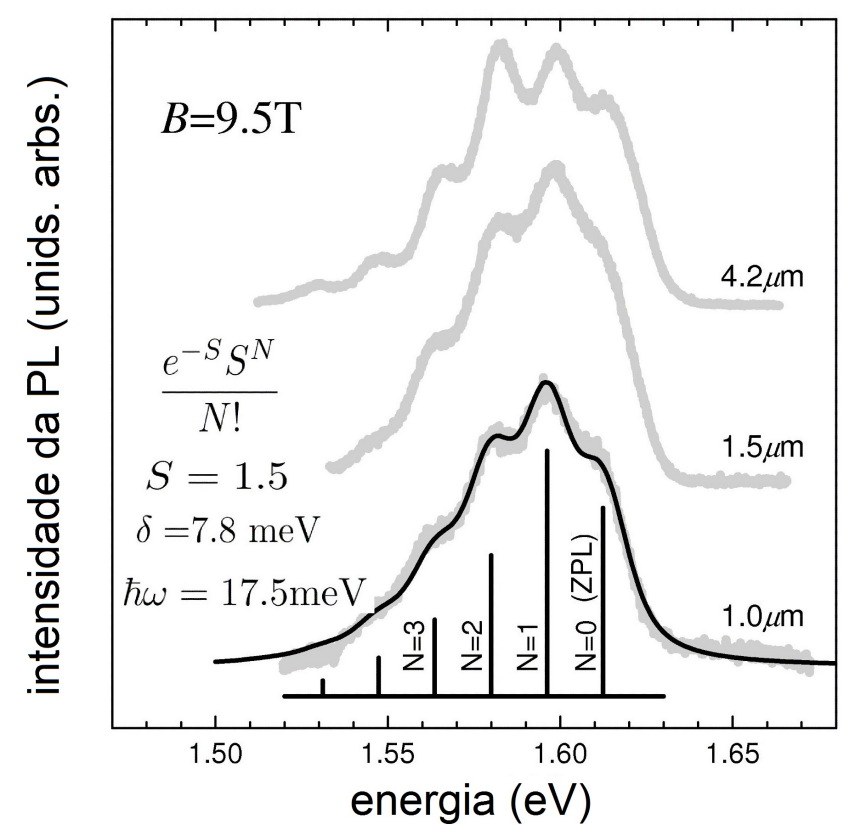

Figura 4.34: Luminescência $\mathrm{MX}_{0}$ para diferentes espessuras da camada epitaxial em $B=9.5 \mathrm{~T}$. A linha preta mostra o ajuste da distribuição de Poisson de intensidades para a PL da amostra mais fina.

gerada por uma soma de gaussianas de intensidades $I$ dadas por (4.200) para o $N$-ésimo modo vibracional. Essa envoltória está representada na 4.34, pela linha preta sobreposta ao espectro de PL da amostra mais fina. Os parâmetros extraídos do ajuste foram $S=1.5$ para a força do acoplamento, $\delta=7.8 \mathrm{meV}$ para a largura das linhas individuais e $\hbar \omega=17.5$ meV para o espaçamento entre as linhas. A partir do ajuste foi possível determinar a posição da linha zero-fônon (ZPL, do inglês zero-phonon line), que corresponde a uma transição que não produz nenhum quantum vibracional. Através dessa linha muito estreita, é possível caracterizar a emissão $\mathrm{MX}_{0}$, acompanhando com precisão o deslocamento da ZPL em função do campo magnético aplicado e da temperatura.

Para compreender o deslocamento da PL com o campo, um gráfico da posição da ZPL em função do campo foi elaborado para três amostras diferentes. O resultado pode ser visto na figura 4.35, que evidencia o enorme contraste entre o deslocamento do gap de absorção e o da posição da ZPL em função do campo magnético externo: um é linear e outro parabólico. Para entender essa diferença, observamos que no processo 


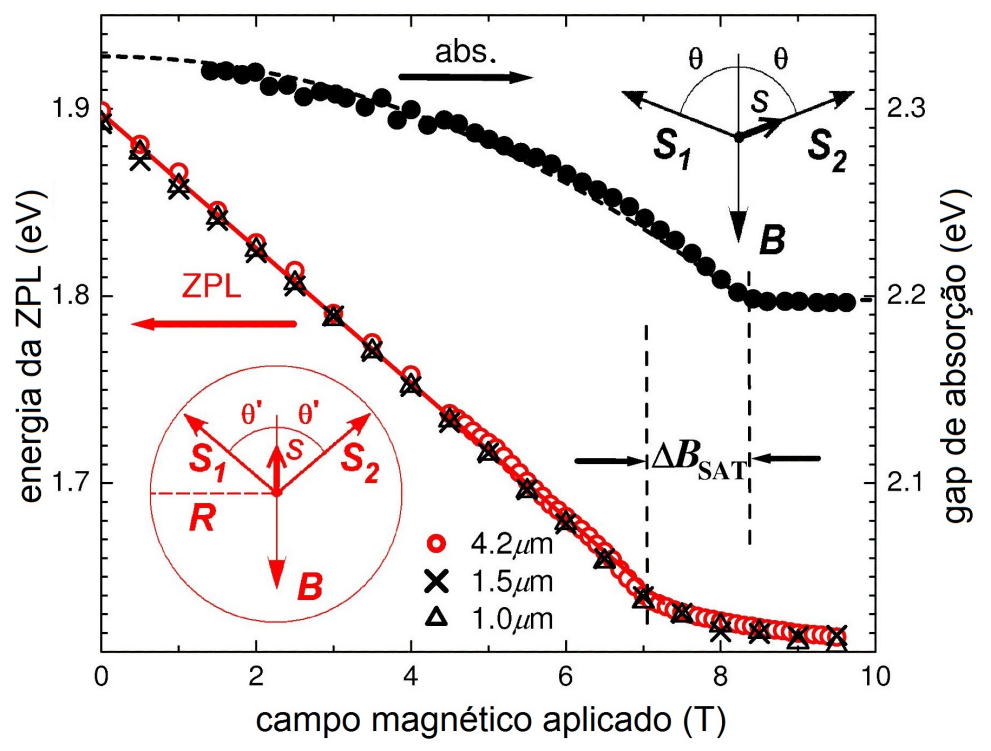

Figura 4.35: Comparação entre o deslocamento da energia do gap e a posição da ZPL em função do campo magnético aplicado.

de absorção, o spin do elétron fotoexcitado preserva a orientação original, paralela a uma das sub-redes, o que leva ao deslocamento quadrático das linhas de absorção com $B$, como deduzido em (4.115). Em contraposição, o processo de recombinação de um elétron em um estado excitado no EuTe tem um tempo característico da ordem de 1 ns [91], o que é tempo suficiente para que o elétron fotoexcitado, que está em contato com o restante do cristal, reduza a sua energia relaxando para um estado $X$, antes que ocorra a recombinação e a emissão de um fóton. Durante a relaxação, o spin eletrônico pode mudar sua orientação para uma direção de mínima energia, a qual é determinada pela energia de exchange $X-f$ entre o elétron fotoexcitado no estado $X$ e os spins da rede. A mesma formulação utilizada em (4.106) para a energia de exchange $d-f$ de um elétron no estado $5 d$, pode ser utilizada para o elétron no estado $X$, substituindo-se o índice $d$ pelo índice $X$

$$
\mathcal{H}_{X f}=-\frac{2 J_{X f}}{\mathcal{N}} \sum_{i}^{\mathcal{N}} s_{X} \cdot S_{i}
$$

Vamos denotar por $\phi$ a direção final do spin $s_{X}$, e por $\theta$ o ângulo entre as sub-redes e a direção do campo, como ilustrado na figura 4.36. Nessa configuração, a equação 
(4.201) resulta em

$$
\mathcal{H}_{X f}=-\frac{2}{\mathcal{N}} J_{X f} s_{X} \cdot\left(\frac{\mathcal{N}}{2} S_{1}+\frac{\mathcal{N}}{2} S_{2}\right)=-\frac{1}{2} J_{X f} S[\cos (\theta-\phi)+\cos (\theta+\phi)]
$$

O ângulo $\phi$ é determinado pelo mínimo de (4.202) em relação a $\phi$, que pode ser obtido

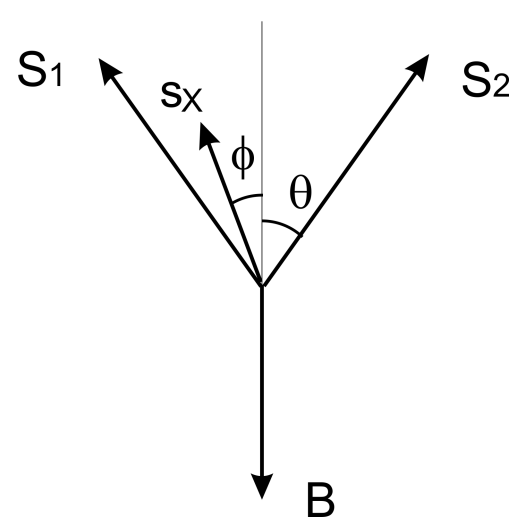

Figura 4.36: O spin $s_{X}$ do elétron fotoexcitado realinha-se para uma direção dada pelo ângulo $\phi$.

igualando-se o derivativo $\frac{\partial \mathcal{H}_{x f}}{\partial \phi}$ a zero

$$
-\frac{1}{2} J_{X f} S[\operatorname{sen}(\theta-\phi)-\operatorname{sen}(\theta+\phi)]=0 \rightarrow \operatorname{sen} \phi \cos \theta=0 \rightarrow \phi=0
$$

Este resultado nos diz que a direção de mínima energia do spin do elétron fotoexcitado é a direção intermediária às sub-redes, como ilustrado na figura 4.37. Substituindo

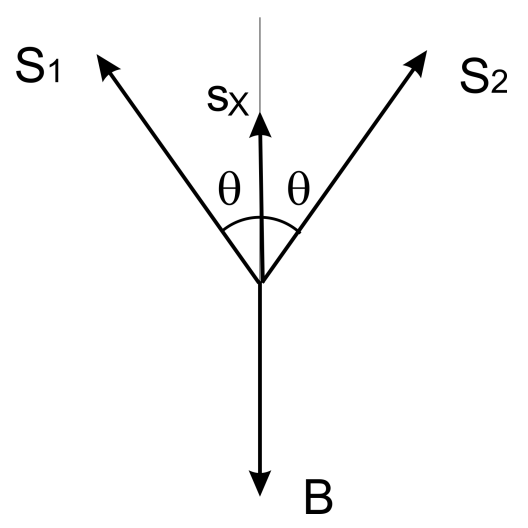

Figura 4.37: A direção do spin do elétron que relaxou para o estado $X$ é a intermediária à direção das sub-redes. 
$\phi=0$ em (4.202), obtemos a energia de exchange entre o elétron fotoexcitado no estado $X$ e os spins da rede

$$
\mathcal{H}_{\text {Xf }}=-J_{X f} S \cos \theta
$$

A presença de um elétron fotoexcitado equivale a um campo magnético efetivo adicional, $B_{X}$, que atua sobre os spins da rede. Admitindo que o recobrimento da função de onda do elétron no estado $X$ seja limitado a $\mathcal{N}$ sítios de európio, com amplitude constante em cada sítio, na presença do elétron fotoexcitado a energia de um spin da rede é acrescida em

$$
\Delta_{X}=\frac{\mathcal{H}_{X f}}{\mathcal{N}}=-\frac{1}{\mathcal{N}} J_{X f} S \cos \theta
$$

Esse termo deve ser somado à energia de um spin $\mathrm{Eu}^{2+}$ deduzida em (4.111), de forma que a energia de um spin da rede na presença de um campo magnético e do elétron fotoexcitado é

$$
E=-g \mu_{B} S B \cos \theta-6 J_{1} S^{2}-6\left(J_{1}+J_{2}\right) S^{2} \cos 2 \theta-\frac{1}{\mathcal{N}} J_{X f} S \cos \theta
$$

A minimização da equação (4.206) em relação a $\theta$ resulta em

$$
\cos \theta=\frac{B+B_{X}}{B_{S A T}}
$$

onde

$$
B_{X}=\frac{J_{X f}}{\mathcal{N} g \mu_{B}}
$$

Este resultado mostra que a presença do elétron no estado $X$, que resulta num campo magnético efetivo $B_{X}$ que atua sobre os spins da rede, faz com que o campo total $B+B_{X}$ que atua sobre os sítios no interior do volume de recobrimento, seja maior do que na ausência do elétron fotoexcitado e portanto a saturação é atingida para um campo aplicado menor. A redução na energia total em função do campo aplicado $B$ pode ser obtida substituindo-se $\cos \theta$ em (4.204) pela expressão obtida em (4.207) para obtermos

$$
\Delta E^{P L}= \begin{cases}-J_{X f} S \frac{B+B_{X}}{B_{S A T}}, & B+B_{X}<B_{S A T} \\ -J_{X f} S, & B+B_{X} \geqslant B_{S A T}\end{cases}
$$


Isso explica a dependência linear do deslocamento das linhas com o campo externo observada na figura 4.35, de onde podemos determinar o deslocamento máximo

$$
\Delta E_{\mathrm{MAX}}^{P L}=J_{X f} S=270 \mathrm{meV}
$$

o qual é atingido para uma magnitude de campo aplicado de $7.1 \mathrm{~T}$, o que é inferior à magnitude necessária para saturar o deslocamento das linhas de absorção. A diferença $\Delta B=1.2$ T é atribuída ao campo efetivo de elétron fotoexcitado, de forma que podemos estimar

$$
B_{X}=1.2 \mathrm{~T}
$$

o que a partir da equação (4.208) resulta em

$$
\mathcal{N}=\frac{J_{X f}}{g \mu_{B} B_{X}} \simeq 555
$$

sítios de Eu no interior do volume de recobrimento da função de onda do elétron fotoexcitado.

A ocorrência da saturação em magnitude inferior à observada na absorção pode ser explicada pelo fato de que o campo efetivo do elétron fotoexcitado favorece o alinhamento dos spins contidos em seu volume de ação. Como no estado $X$ a direção do campo efetivo coincide com a direção do campo externo, que é a direção entre as sub-redes, os campos externo e efetivo são somados, sendo necessário aplicar uma magnitude menor de campo para saturar magneticamente o sistema. Mesmo em campo nulo, o campo efetivo $B_{X}$ define uma região de spins polarizados ao redor do elétron fotoexcitado, o que pode ser interpretado por meios de uma quasi-partícula denominada polaron magnético, a qual será o tema da seção seguinte. Os resultados das medidas da PL do EuTe que permitiram detectar a formação de polarons magnéticos, especificamente os apresentados nas figuras 4.28, 4.34 e 4.35, foram publicados na referência [92].

\subsubsection{Polaron magnético no EuTe}

Na seção 4.3 vimos que a presença do elétron fotoexcitado origina um campo $B_{X}$ que atua sobre os spins da rede, favorecendo um alinhamento dos spins no interior de 
seu volume de ação, formando um polaron magnético. A seguir iremos determinar, a partir dos resultados experimentais, alguns dos parâmetros desse polaron, como seu raio, magnetização e energia de ligação.

Através da magnitude de $B_{X}$, dada por (4.211), é possível estimar o tamanho do polaron como se segue. Vamos supor para o estado $X$ uma função de onda esférica de raio $R$, que recobre um número $\mathcal{N}$ de sítios da rede. A densidade de sítios da rede $f c c$ é dada por $N=\frac{4}{a^{3}}$, onde $a$ é o parâmetro de rede. Com isso temos

$$
\mathcal{N}=N \times V=\frac{4}{a^{3}} \times \frac{4}{3} \pi R^{3}
$$

Substituindo (4.213) em (4.208) obtemos

$$
B_{X}=\frac{J_{X f}}{\mathcal{N} g \mu_{B}}=\frac{J_{X f}}{g \mu_{B}} \frac{3}{16 \pi}\left(\frac{a}{R}\right)^{3}
$$

e portanto

$$
R=\left(\frac{3}{16 \pi} \frac{J_{X f}}{g \mu_{B} B_{X}}\right)^{\frac{1}{3}} a
$$

Com os valores experimentais $B_{X}=1.2 \mathrm{~T}$ e $J_{X f} S=270 \mathrm{meV}$ de deslocamento máximo da $\mathrm{ZPL}$, o raio do polaron pode ser estimado como

$$
R=3.6 a
$$

Como visto na seção 4.3 , em $B=7.1 \mathrm{~T}$, a reta da posição da ZPL em função do campo muda de derivada, indicando a saturação magnética dos spins da rede no interior do polaron. Entretanto, o elétron fotoexcitado continua a reduzir ligeiramente sua energia, como pode ser visto por exemplo na figura 4.31. Uma possível justificativa para essa adicional redução da energia é a que segue: o elétron fotoexcitado reduz sua energia cinética aumentando seu raio de ação, entretanto o aumento do raio implica num custo de energia para alinhar os spins da rede no interior do polaron, de forma que o ganho energético é dado pelo balanço

$$
\Delta E(R)=\frac{\hbar^{2}}{2 m_{e} R^{2}}+\frac{4}{3} \pi R^{3} N \delta U
$$


onde $N$ é a densidade de sítios da rede e $\delta U$ é a energia necessária para alinhar um spin da rede. A aplicação do campo magnético faz com que os spins da rede alinhem-se à direção do campo, de forma que a energia $\delta U$ necessária para alinhar um spin da rede seja reduzida, causando o desvio das linhas de PL para campos entre $B=7.1$ e $B=8.3 \mathrm{~T}$.

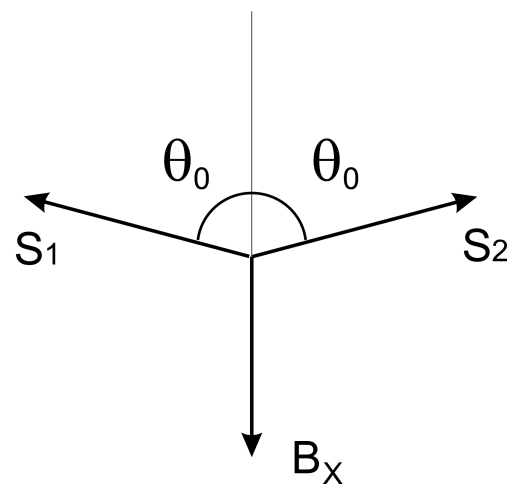

Figura 4.38: Ângulo de inclinação dos spins em $B=0$.

Em campo externo nulo, os spins no interior do polaron estão sujeitos à ação do campo efetivo $B_{X}$, o qual é muito maior que $B_{s f}$, e portanto faz com que esses spins sejam inclinados na direção de $B_{X}$, formando um ângulo $\theta_{0}$ com essa direção (figura 4.38). Esse ângulo pode ser estimado substituindo-se $B=0$ em (4.207) o que resulta em

$$
\cos \theta_{0}=\frac{B_{X}}{B_{S A T}}=\frac{1.2}{7.2} \rightarrow \theta_{0}=80.4^{\circ}
$$

onde foi utilizado o valor conhecido do campo de saturação do EuTe $B_{S A T}=7.2$ T [67]. Cada um dos sítios no interior do polaron possui um momento magnético efetivo $M_{S} \cos \theta_{0}$, de forma que a magnetização do polaron é dada por $M_{\mathrm{p}}=\mathcal{N} M_{S} \cos \theta_{0}$ o que $\operatorname{com} R=3.6 a$ resulta em

$$
M_{\mathrm{p}} \approx 780 \mu_{B}
$$

A energia de ligação do polaron pode ser estimada através do ganho energético máximo do sistema devido à formação do polaron, o qual é dado por (4.209) e para $B=0 \mathrm{~T}$ e $T=0 \mathrm{~K}$ vale

$$
E_{p}=-J_{X f} S \frac{B_{X}}{B_{S A T}}
$$


Com os valores $J_{X f} S=270 \mathrm{meV}, B_{X}=1.2 \mathrm{~T}$ deduzidos anteriormente nas equações (4.210) e (4.211), respectivamente e com $B_{S A T}=7.2 \mathrm{~T}$ (como na referência [67]), obtemos

$$
E_{p}=45 \mathrm{meV}
$$

Essa configuração é consistente com o polaron magnético do tipo II, previsto para o EuTe na referência [93] e ilustrado na figura 4.39 a) para $B=0 \mathrm{~T}$, onde os spins da rede no interior do polaron formam um ângulo $\theta_{0}$ com a direção do spin do elétron fotoexcitado e na figura $4.39 \mathrm{~b}$ ) para $B>0$, onde os spins no interior do polaron estão inclinados na direção do campo com um ângulo $\theta$ inferior ao ângulo de inclinação dos spins fora do polaron.

a)

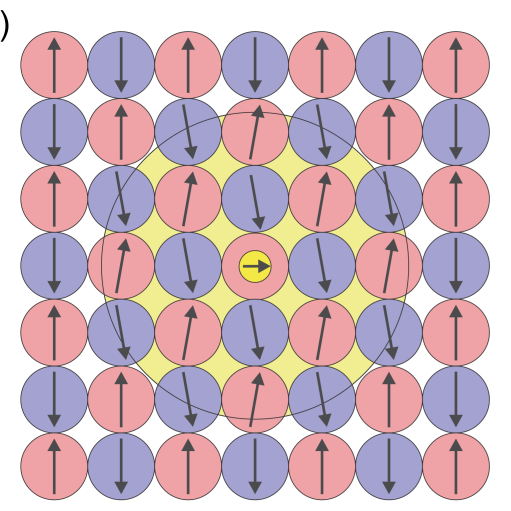

b)

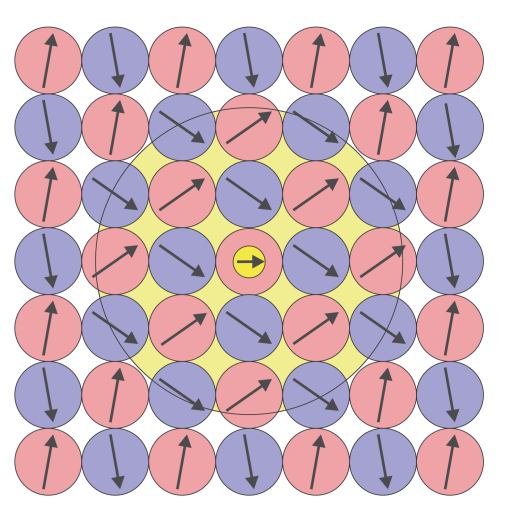

$B$

Figura 4.39: Polaron magnético do tipo dois a) em campo nulo b) com um campo magnético perpendicular ao eixo AFM. Uma região da rede tem seus spins inclinados à direção do spin do elétron fotoexcitado.

\subsubsection{Deslocamento das linhas de absorção e PL em termos da mag- netização}

Os resultados (4.115) e (4.209) para o deslocamento das linhas de absorção e PL também podem ser exprimidos em termos da magnetização da rede, através do ângulo $\theta$ entre as sub-redes. 
Em $T=0 \mathrm{~K}$, as duas sub-redes, de magnetizações $M_{1}=M_{S} / 2$ e $M_{2}=M_{S} / 2$ fazem um ângulo $\theta$ com a direção do campo, como mostrado na figura 4.40 a). A magnetização

a)

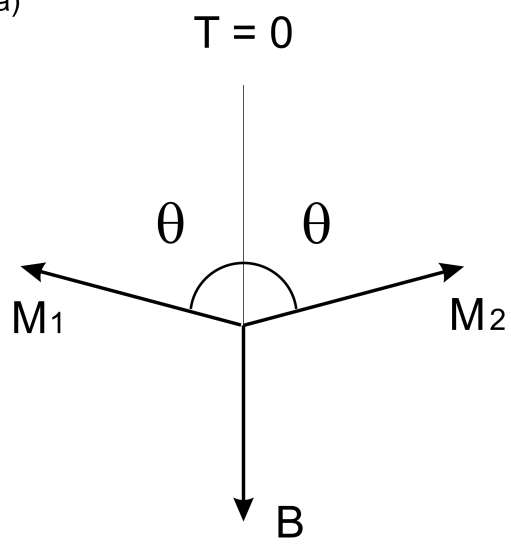

b)

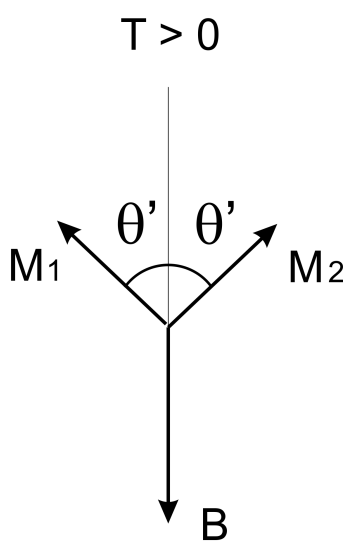

Figura 4.40: Magnetização das sub-redes sob ação do campo efetivo em $T=0 \mathrm{~K}$.

total, é dada pelas componentes na direção do campo

$$
M=M_{1} \cos \theta+M_{2} \cos \theta=M_{S} \cos \theta
$$

de forma que

$$
\cos \theta=\frac{M}{M_{S}}
$$

Substituindo esse resultado em (4.115) e (4.209) obtemos

$$
\Delta E^{\mathrm{ABS}}=-J_{d f} S\left(\frac{M}{M_{S}}\right)^{2}=-\Delta E_{\mathrm{MAX}}^{\mathrm{ABS}}\left(\frac{M}{M_{S}}\right)^{2}
$$

$\mathrm{e}$

$$
\Delta E^{P L}=-J_{X f} S \frac{M}{M_{S}}=\Delta E_{\mathrm{MAX}}^{P L} \frac{M}{M_{S}}
$$

Os resultados acima podem ser utilizados para obter a dependência do deslocamento das linhas de absorção e PL em função da temperatura, onde a temperatura é introduzida através da magnetização.

Para $0<T<T_{N}$, as flutuações térmicas causam uma redução na magnitude de $S$, que pode ser contabilizada pelo valor médio $\langle S\rangle$. Ao mesmo tempo, as flutuações 
térmicas reduzem o campo molecular, causando uma redução no ângulo $\theta$ entre a direção do campo e as direções de $M_{1}$ e $M_{2}$ para um valor $\theta^{\prime}$ (ver figura 4.40 b)). A magnetização total é dada pelas componentes das magnetizações de cada sub-rede na direção do campo

$$
M=N g \mu_{B}\langle S\rangle \cos \theta^{\prime} \rightarrow \cos \theta^{\prime}=\frac{M}{N g \mu_{B}\langle S\rangle}=\frac{S M}{\langle S\rangle M_{S}}
$$

Para as linhas de PL,

$$
\Delta E^{\mathrm{PL}}=-J_{X f} S \cos \theta \rightarrow-J_{X f}\langle S\rangle \cos \theta^{\prime}=-J_{X f}\langle S\rangle \frac{S M}{\langle S\rangle M_{S}}=-\Delta E_{\mathrm{MAX}}^{P L} \frac{M}{M_{S}}
$$

mostrando que, assim como para $T=0 \mathrm{~K}$, o deslocamento é linear com $M$.

Em temperaturas suficientemente altas, quando as magnetizações de todas as subredes estão orientadas na direção do campo, $\cos \theta=1$. O valor médio de $S$ é muito inferior a seu valor em $T=0 \mathrm{~K}$ e pode ser obtida através da magnetização total $M=$ $N g \mu_{B}\langle S\rangle$ como $\langle S\rangle=\frac{S M}{M_{S}}$. Dessa forma

$$
\begin{aligned}
\Delta E^{\mathrm{ABS}}= & -J_{d f} S \cos ^{2} \theta \rightarrow-J_{d f} \frac{S M}{M_{S}} \\
& \therefore \Delta E^{\mathrm{ABS}}=-\Delta E_{\mathrm{MAX}}^{\mathrm{ABS}} \frac{M}{M_{S}}
\end{aligned}
$$

$\mathrm{e}$

$$
\begin{aligned}
\Delta E^{\mathrm{PL}}= & -J_{X f} S \cos \theta \rightarrow-J_{X f} \frac{S M}{M_{S}} \\
& \therefore \Delta E^{\mathrm{PL}}=-\Delta E_{\mathrm{MAX}}^{P L} \frac{M}{M_{S}}
\end{aligned}
$$

\subsubsection{Evidências da formação de polarons magnéticos em EuTe a par- tir de um estudo da ZPL em função da temperatura}

Na seção anterior, a partir de medidas da ZPL em função do campo magnético a uma temperatura fixa, obtivemos a primeira evidência da formação de polarons magnéticos em EuTe. Com os resultados obtidos foi possível determinar o tipo de polaron, sua energia de ligação e seu raio característico. Nesta seção, procuraremos reforçar esses 
resultados a partir de medidas da PL em função da temperatura, onde será possível obter uma estimativa independente da energia de ligação do polaron, que deve desaparecer em temperaturas altas, onde o campo $B_{X}$ não é suficiente para alinhar os spins no raio de ação do elétron fotoexcitado. O aumento da temperatura diminui a magnetização da rede, o que de acordo com os resultados (4.227) e (4.231) causa uma redução no ganho energético por exchange, que é refletido em um deslocamento para o azul da ZPL com o aumento da temperatura. Observe-se que todo o deslocamento para o azul da ZPL se deve unicamente à interação de exchange, dado que modelagens teóricas indicam que a distribuição radial da função de onda do elétron fotoexcitado (e por consequência o raio do polaron), não sofrem nenhuma alteração com variações da temperatura em um intervalo de $T=T_{N}$ a $T=10 T_{N}$ [36], assim as energias cinética e potencial do elétron permanecem constantes neste intervalo de temperaturas. A relação entre $\Delta E^{P L}$ e a magnetização foi obtida na seção 4.3.2, o que torna trivial incluir a dependência térmica em $\Delta E^{P L}$ considerando-se que a magnetização é uma função da temperatura.

Em temperaturas altas, o sinal da luminescência é muito fraco e por isso foi necessário procurar a potência máxima de excitação para a qual ainda não sejam excitados os estados associados às linhas $\mathrm{MX}_{1}$ e $\mathrm{MX}_{2}$. A figura 4.41 mostra a $\mathrm{PL}$ em função da temperatura para duas diferentes potências de excitação. Para a potência de 24 $\mathrm{mWcm} /{ }^{2}$ é possível observar um pequeno máximo de intensidade que surge à direita da banda $\mathrm{MX}_{0}$ a partir de $\mathrm{T} \sim 14 \mathrm{~K}$. Esse máximo, o qual é associado à linha $\mathrm{MX}_{1}$, também desloca-se para o azul com o aumento da temperatura, entretanto é indesejável para a obtenção precisa da posição da ZPL em função da temperatura. Por isso foi utilizada uma potência máxima de $2,4 \mathrm{mWcm} /{ }^{2}$, cujos espectros não apresentam nenhum máximo que possa ser associado à linha $\mathrm{MX}_{1}$.

Determinada a máxima potência de excitação para que a linha $\mathrm{MX}_{1}$ não seja excitada, os espectros de PL em função da temperatura foram medidos em duas amostras diferentes, de espessuras 1.0 e $1.5 \mu \mathrm{m}$, para as quais o efeito de interferência de Fabry-Perot não influencia a forma das curvas. Para obter com maior precisão o deslocamento $\Delta E_{P L}$, 


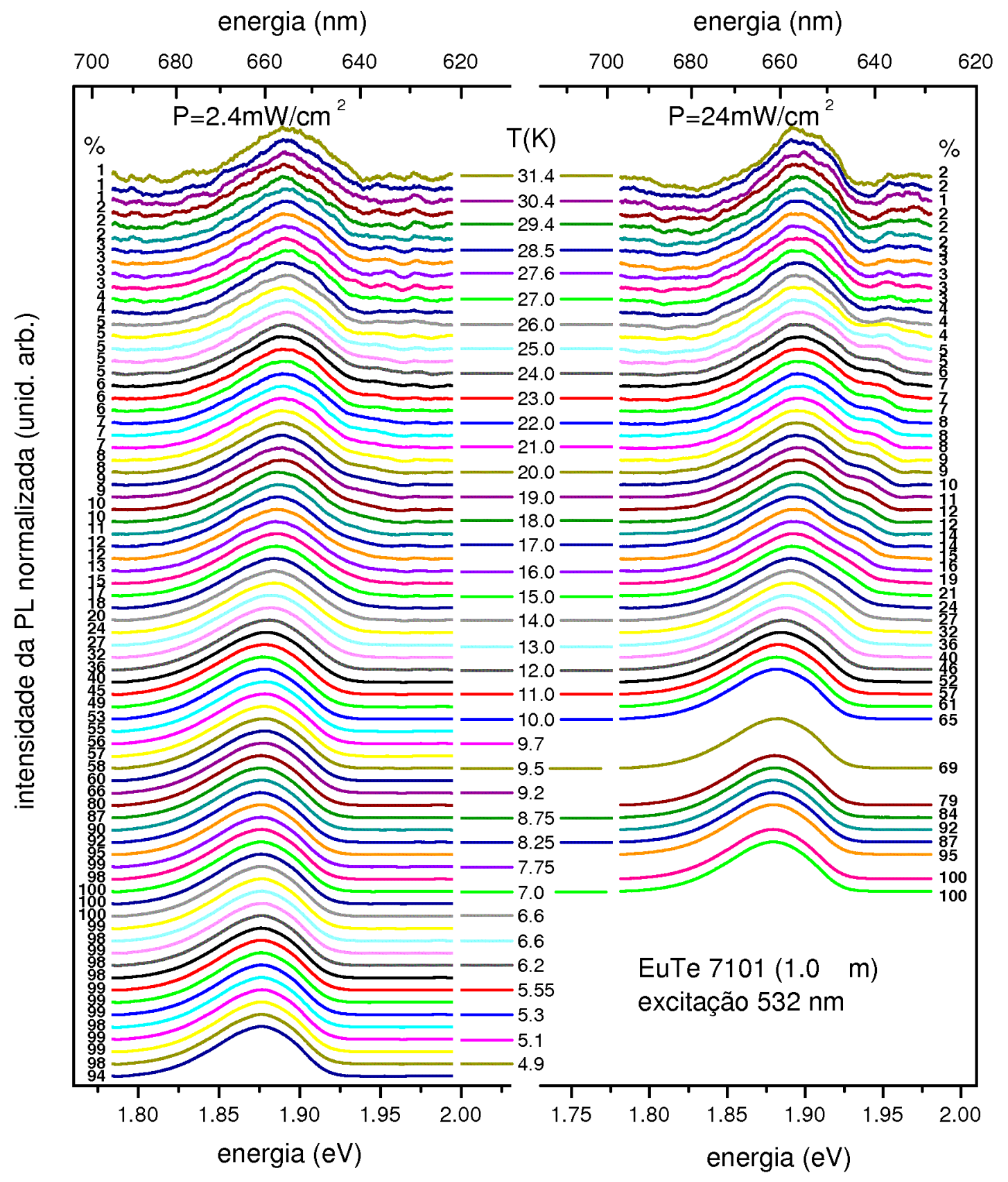

Figura 4.41: $\mathrm{PL} \mathrm{MX}_{0}$ em função da temperatura para duas potências diferentes. Um desvio para o azul pode ser observado na posição das linhas.

a posição da ZPL foi acompanhada a partir da segunda derivada das curvas espectrais para temperaturas no intervalo de 4.8 até $30 \mathrm{~K}$ com passo de $0.1 \mathrm{~K}$. Essas curvas estão apresentadas na figura 4.42 para a amostra de $1.0 \mu \mathrm{m}$, em escala logarítmica, onde 
a linha azul indica o máximo associado à ZPL nas temperaturas em que é possível identificá-lo.

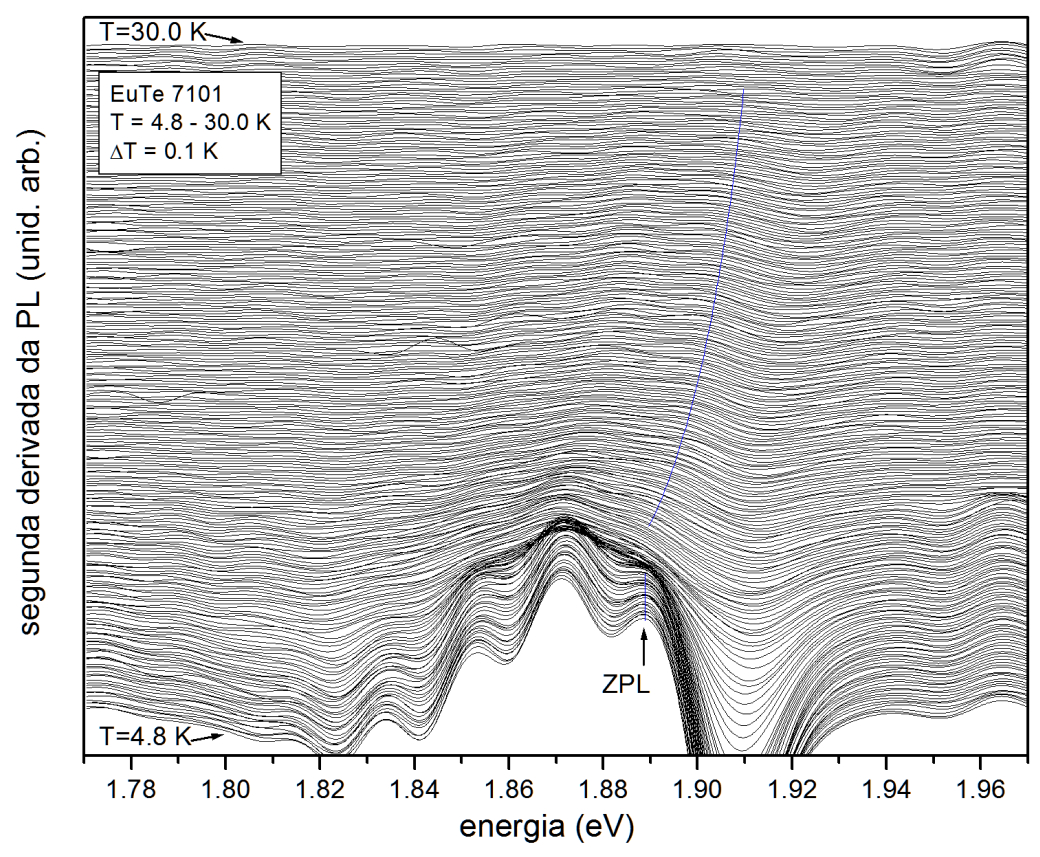

Figura 4.42: Segunda derivada da PL em função da temperatura.

Em temperaturas baixas o sinal é muito intenso e é possível determinar com facilidade a posição da ZPL. Na medida que aumenta-se a temperatura, o sinal perde intensidade caindo pela metade em $T=10 \mathrm{~K}$ e para $1 \%$ em $T=30 \mathrm{~K}$. Entretanto, olhando para cada curva individualmente, ainda é possível distinguir os picos associados à ZPL, exceto no intervalo de temperaturas ao redor de $T_{N}$, onde o comportamento crítico do sistema impede a definição de um pico. Isto ocorre porque em $T<T_{N}$, o sistema encontra-se na fase AFMII, de forma que há uma homogeneidade na estrutura magnética que garante que todos os polarons formados sejam idênticos. Entretanto, em $T=T_{N}$ a existência de flutuações térmicas que introduzem perturbações aleatórias nos spins da rede que podem fazer com que haja polarons de diferentes energias, $\mathrm{o}$ que reflete-se num alargamento da ZPL, que fica impossível de ser determinada em temperaturas próximas de $T_{N}$. Em temperaturas acima de $T_{N}$, as flutuações térmicas tornam-se muito frequentes, de forma que na média as mesmas atuam de maneira 
equivalente em todos os polarons, e o sistema volta a ser homogêneo, o que permite novamente associar um máximo na segunda derivada da PL à ZPL. Na figura 4.43 vemos as segundas derivadas normalizadas das curvas de PL da amostra de $1.0 \mu \mathrm{m}$ para algumas temperaturas. É possível perceber que na região de temperaturas entre $\sim 9$ e $15 \mathrm{~K}$ não é possível definir-se o máximo associado à ZPL. Acima de $15 \mathrm{~K}$ é possível novamente identificar a posição da ZPL, entretanto, devido à diminuição na intensidade da PL, o sinal vai tornando-se cada vez mais ruidoso com o aumento da temperatura, de forma que a incerteza na posição da ZPL também aumenta com a temperatura.

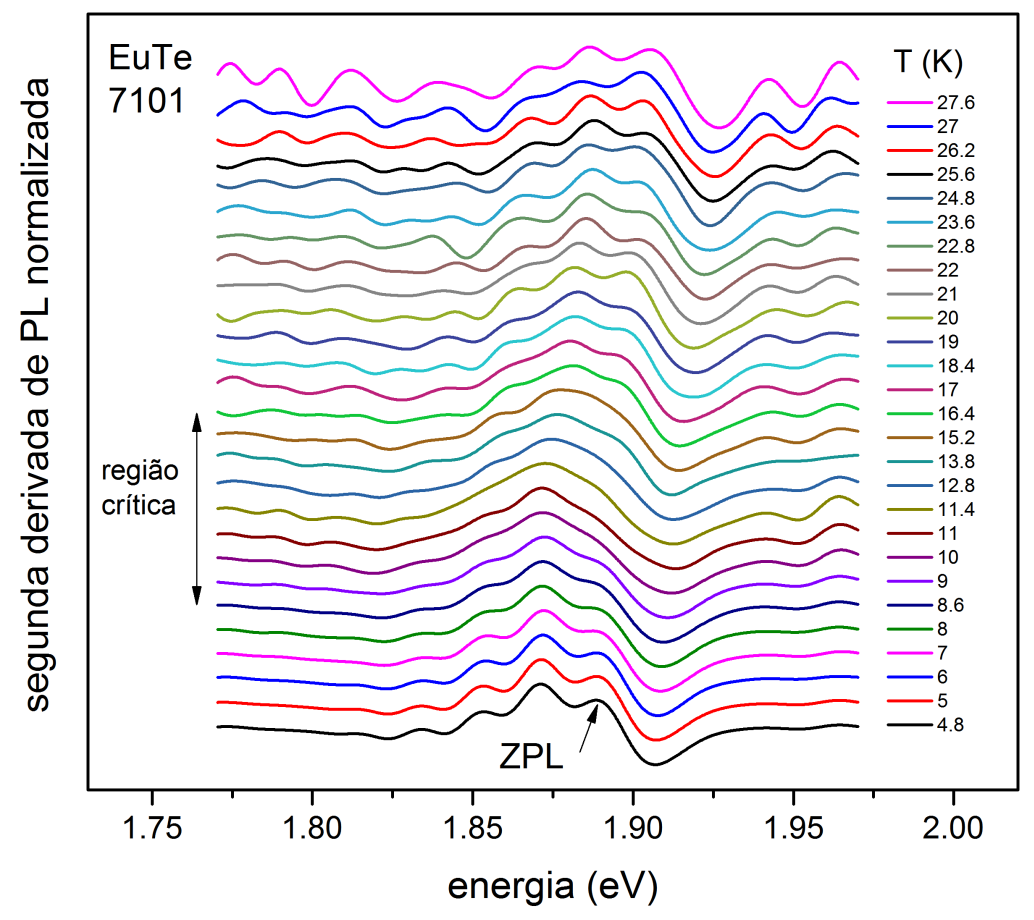

Figura 4.43: Segunda derivada normalizada da PL para algumas temperaturas. Nota-se que na região entre T $\sim 9 \mathrm{~K}$ e T $\sim 15 \mathrm{~K}$ não é possível identificar a posição da ZPL.

A posição da ZPL em função da temperatura para duas amostras, de espessuras de $1.0 \mu \mathrm{m}$ e de $1.5 \mu \mathrm{m}$, é apresentada na figura 4.44, para as temperaturas em que foi possível identificar a ZPL. Claramente há um desvio para o azul a partir de $T_{N}$, que está diretamente relacionado com a redução da magnetização no interior raio polarônico com o aumento da temperatura. 


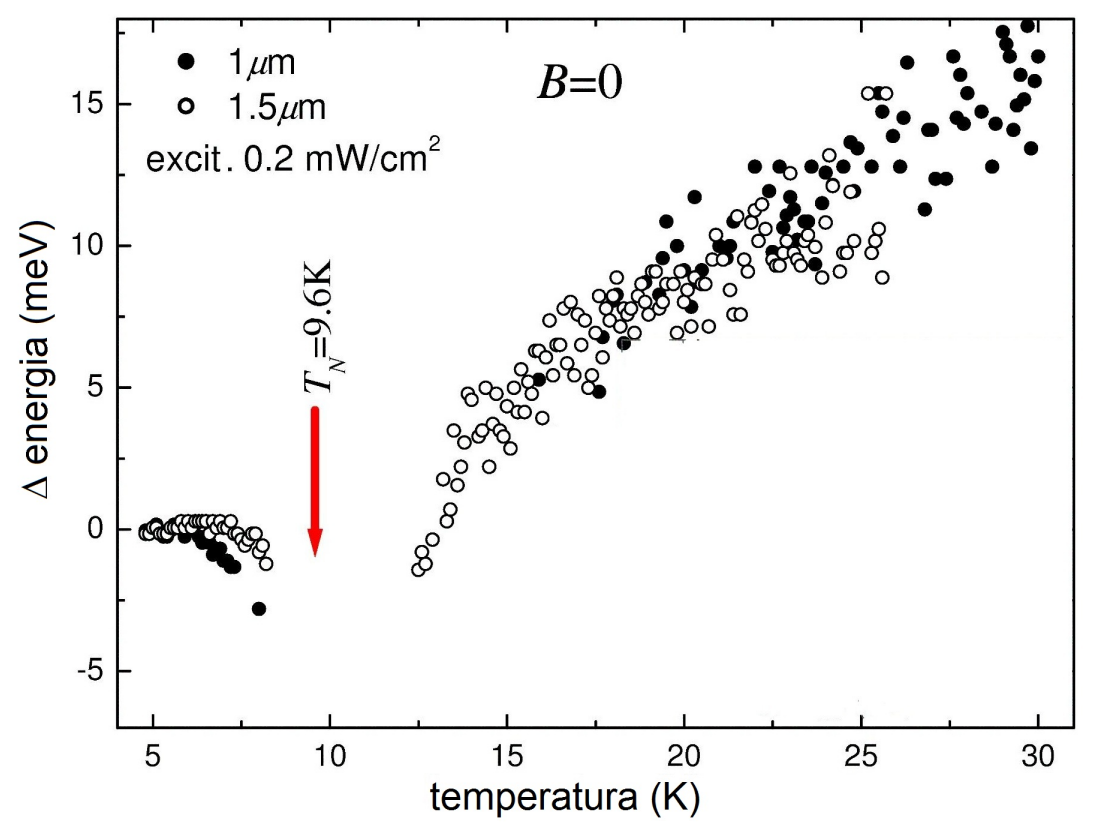

Figura 4.44: Posição da ZPL determinada a partir da segunda derivada das curvas espectrais para duas amostras diferentes.

A magnetização de um antiferromagneto é bem conhecida, e determinada pelas susceptibilidades $\chi^{\|}$e $\chi^{\perp}$, de acordo com a direção do campo externo em relação ao eixo AFM. Se o campo é aplicado paralelo ao eixo AFM, esse tende a aumentar a magnetização de uma sub-rede e diminuir na mesma proporção a magnetização da outra, como mostra a figura 4.45 a). Em $T=0 \mathrm{~K}$, as sub-redes encontram-se saturadas e não há nada que o campo possa fazer, portanto $\chi^{\|}=0$. Quando aumenta-se a temperatura, as flutuações térmicas reduzem o campo molecular e o campo passa a ser capaz de alinhar uma parcela dos spins para a sua direção, parcela esta tanto maior quanto for a temperatura, de forma que $\chi^{\|}$aumenta suavemente com a temperatura até $T_{N}$.

Se o campo for aplicado perpendicular ao eixo AFM, as magnetizações das subredes tendem a inclinarem-se para a direção do campo, como mostra a figura 4.45 b). É possível demonstrar que $\chi^{\perp}$ independe de $T$ abaixo de $T_{N}$, pelos argumentos que seguem: o aumento da temperatura, reduz o módulo da magnetização de cada sub-rede, o que pode ser formulado em termos do valor médio do spin total de cada 
a)

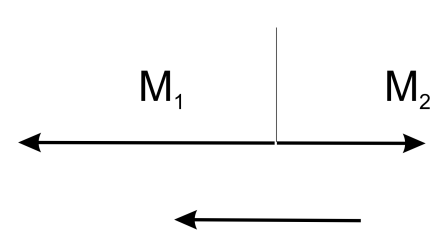

B b)

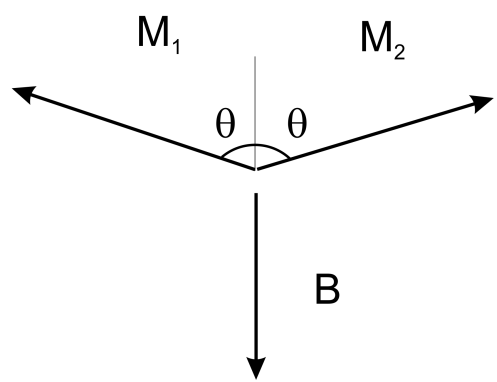

Figura 4.45: a) Campo magnético orientado paralelamente ao eixo AFM. b) Campo magnético orientado perpendicularmente ao eixo AFM.

sub-rede

$$
M_{i}=\frac{N}{2} g \mu_{B} S \rightarrow M_{i}=\frac{N}{2} g \mu_{B}\langle S\rangle
$$

Por outro lado, o ângulo formado entre as sub-redes e a direção do campo, dado pela equação (4.112), também é afetado pela redução da magnitude dos spins da rede com o aumento da temperatura

$$
\cos \theta=\frac{B}{B_{S A T}}=\frac{N g \mu_{B} B}{24\left|J_{1}+J_{2}\right|\langle S\rangle} \rightarrow \cos \theta=\frac{N g \mu_{B} B}{24\left|J_{1}+J_{2}\right|\langle S\rangle}
$$

de forma que a magnetização total, dada por

$$
M=M_{1} \cos \theta+M_{2} \cos \theta=N g \mu_{B}\langle S\rangle \frac{N g \mu_{B} B}{24\left|J_{1}+J_{2}\right|\langle S\rangle}=24\left|J_{1}+J_{2}\right| N^{2} g^{2} \mu_{B}^{2} B
$$

independe da temperatura e portanto $\chi^{\perp}$ é constante no intervalo $0<T<T_{N}$. Note que em $T \rightarrow T_{N}$, quando a magnetização das sub-redes tende a zero, as susceptibilidades $\chi^{\|}$e $\chi^{\perp}$ tornam-se iguais.

Acima da temperatura de Néel, quando o material encontra-se na fase paramagnética, a susceptibilidade independe da direção do campo e é dada pela lei de Curie-Weiss, obtida na seção 2.2.2 e equação (2.126) como

$$
\chi \propto \frac{1}{T+T_{N}}
$$

A figura 4.46 resume essa situação, onde as susceptibilidades $\chi^{\|}$e $\chi^{\perp}$ são apresentadas em função da temperatura e também é apresentada a susceptibilidade de um 
material de múltiplos domínios magnéticos orientados aleatoriamente, que apresenta um comportamento intermediário entre $\chi^{\|}$e $\chi^{\perp}$. Nesse último caso, o campo magnético aplicado irá interagir simultaneamente tanto com spins orientados numa direção perpendicular quanto com spins orientados numa direção paralela. Se o campo for aplicado na direção $z$, por exemplo, existem duas direções perpendiculares $(x$ e $y$ ) ao campo magnético, de forma que a probabilidade de haver spins perpendiculares a $z$ é duas vezes maior do que a probabilidade de haver spins paralelos a $z$. A susceptibilidade total portanto será dada por uma combinação de $\chi^{\|}$e $\chi^{\perp}$ com pesos proporcionais à probabilidade de haver spins paralelos ou perpendiculares a $B$ :

$$
\chi_{T}=\frac{1}{3} \chi^{\|}+\frac{2}{3} \chi^{\perp}
$$

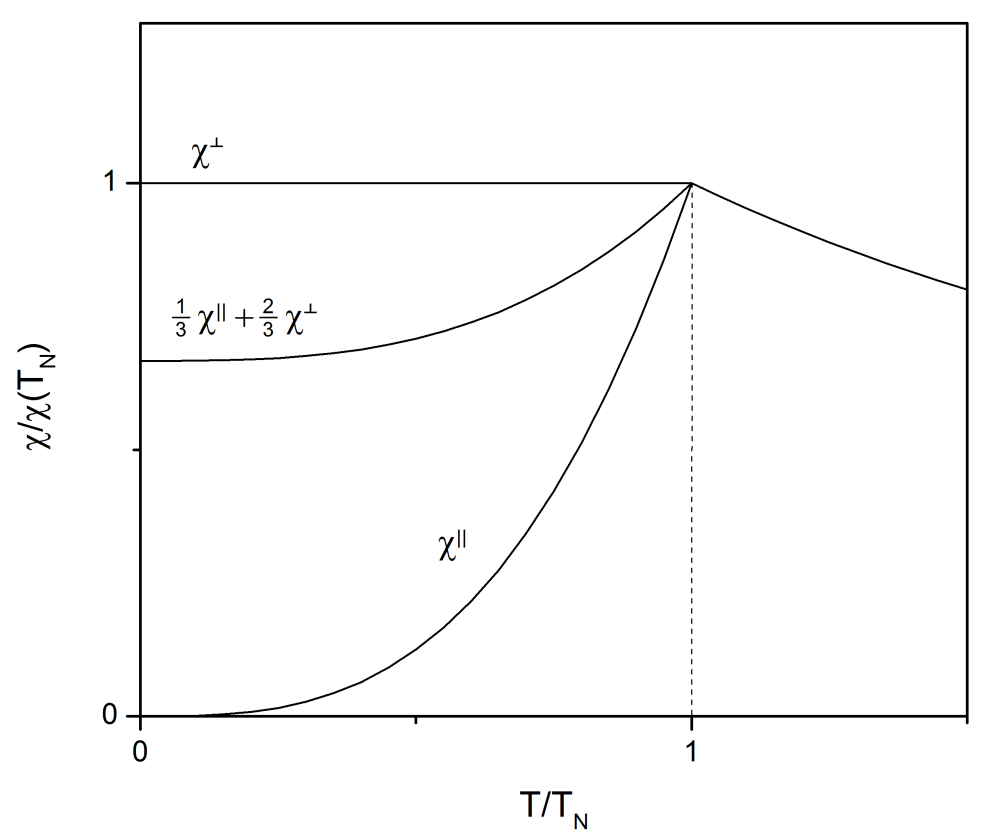

Figura 4.46: Susceptibilidade AFM

Voltando para o EuTe, para $T>T_{N}$ o sistema encontra-se na fase paramagnética. Nessa região de temperaturas, fora do volume do polaron, os spins da rede tem suas direções distribuídas aleatoriamente e a magnetização total é nula. No interior do polaron, o campo efetivo $B_{X}$ favorece o alinhamento dos spins à sua própria direção, de 
forma que na média, cada spin da rede tem uma componente na direção de $B_{X}$ dada por $\langle S\rangle$, como ilustrado na figura 4.47. O aumento da temperatura implica numa diminuição da susceptibilidade e portanto da magnetização induzida pelo elétron fotoexcitado, reduzindo assim o ganho de energia de exchange devido à formação do polaron, o que, como observamos na figura 4.44 b) está de acordo com o comportamento da posição da ZPL em função da temperatura para $T>T_{N}$.

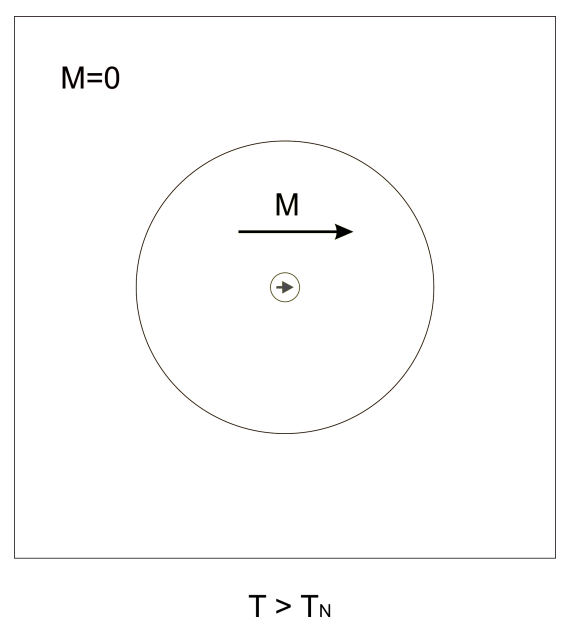

Figura 4.47: Pólaron magnético nos EuX na fase paramagnética.

Para $T<T_{N}$, o campo efetivo do elétron fotoexcitado é orientado na direção intermediária às sub-redes, como visto anteriormente (figura 4.38), portanto seria de se esperar que a magnetização induzida pelo elétron fotoexcitado fosse determinada por $\chi^{\perp}$. Entretanto, claramente a figura 4.44 b) mostra que a magnetização induzida pelo elétron fotoexcitado tem um caráter intermediário entre $\chi^{\|} \mathrm{e} \chi^{\perp}$. Um explicação plausível é de que o polaron contenha no seu interior mais de um domínio magnético, de forma que o campo magnético efetivo seja perpendicular à magnetização de alguns domínios e paralelo à magnetização de outros. Isso só é possível se os domínios forem menores do que o diâmetro característico do polaron, $7.2 a \simeq 50 \AA$, o que estabelece um limite superior para o tamanho esperado dos domínios AFM no EuTe. Entretanto essa possibilidade deve ser descartada, uma vez que o campo efetivo $B_{X}$ é duas ordens de grandeza maior do que o campo spin-flop, pelo que não se deve esperar que existam domínios magnéticos no interior do polaron. 
Uma outra possibilidade mais plausível é a seguinte: a descrição do polaron com uma fronteira abrupta e interação de exchange $X f$ constante no interior do polaron é uma ideia aproximada, pois na realidade o elétron fotoexcitado interage por exchange de forma decrescente com spins mais distantes do que os contidos na esfera de raio $R_{p}$. Nesse caso seria de se esperar alguma contribuição de $\chi^{\|}$ao deslocamento $\Delta E^{P L}$, em função da interação de exchange entre o elétron fotoexcitado e sítios contidos nas bordas do polaron magnético, onde o campo efetivo é inferior a $B_{s f}$ e portanto há domínios magnéticos. Notamos que na figura 4.44 b) a contribuição de $\chi^{\|}$é de cerca de $1 / 4$, o que é inferior aos $1 / 3$ esperados para o caso de uma distribuição aleatória das direções das magnetizações sob o efeito de $B_{X}$. Isto significa que a $\chi^{\perp}$ no interior do polaron é predominante, o que reforça a interpretação de que a contribuição de $\chi^{\|}$à susceptibilidade total advém de um volume menor do que o volume total do polaron, por exemplo a região das bordas.

Tanto para o campo na orientação paralela quanto na direção perpendicular ao eixo AFM, como pode ser visto na figura 4.46, a susceptibilidade magnética é máxima em $T_{N}$, de forma que a magnetização induzida pelo elétron fotoexcitado é máxima nessa temperatura. A partir de $T_{N}$ a susceptibilidade decresce com o aumento da temperatura até que em $T \rightarrow \infty \chi$ vai a zero e não é mais possível magnetizar o sistema. Assim, uma estimativa independente da energia de ligação do polaron pode ser obtida a partir do deslocamento da ZPL entre $T_{N}$ e $T \rightarrow \infty$ em campo nulo.

Na seção 4.3.2 vimos que para $T>0$ o deslocamento das linhas de PL é dado por

$$
\Delta E^{\mathrm{PL}}=-\Delta E_{\mathrm{MAX}}^{P L} \frac{M}{M_{S}}
$$

Por outro lado, foi visto na seção 2.3.2, que a magnetização dos EuX na fase paramagnética é dada pela equação (2.143), que neste caso se reduz a

$$
M=\frac{C B_{X}}{T-\Theta}
$$

Por substituição de (4.238) em (4.237) obtemos para o deslocamento das linhas de PL

$$
\Delta E^{\mathrm{PL}}=-\Delta E_{\mathrm{MAX}}^{P L} \frac{C B_{X}}{M_{S}(T-\Theta)}=-\Delta E_{\mathrm{MAX}}^{P L} \frac{(S+1) g \mu_{B} B_{X}}{3 k_{B}(T-\Theta)}
$$


Substituindo os valores experimentais $\Delta E_{\mathrm{MAX}}^{P L}=270 \mathrm{meV}$ e $B_{X}=1.2 \mathrm{~T}$ obtidos na seção 4.3 e $\Theta=\frac{2 S(S+1)}{3 k_{B}}\left(12 J_{1}+6 J_{2}\right)=-4 \mathrm{~K}$ dado pela referência [19], obtemos um deslocamento máximo da $Z P L$ de $\Delta E^{P L}(\infty)-\Delta E^{P L}\left(T_{N}=9.6\right)=46 \mathrm{meV}$ sem nenhum parâmetro de ajuste, que está em pleno acordo com a energia de ligação obtida na equação (4.221) para $T=0 \mathrm{~K}$. Esses valores são compatíveis na medida que a aproximação do polaron com fronteiras rígidas e função de onda uniforme corresponde à situação em que a magnetização é governada por $\chi^{\perp}$, que é constante para $0<T<T_{N}$. A figura 4.48 mostra a curva determinada por (4.239) sobreposta aos pontos experimentais, onde podemos observar a concordância do modelo com os resultados.

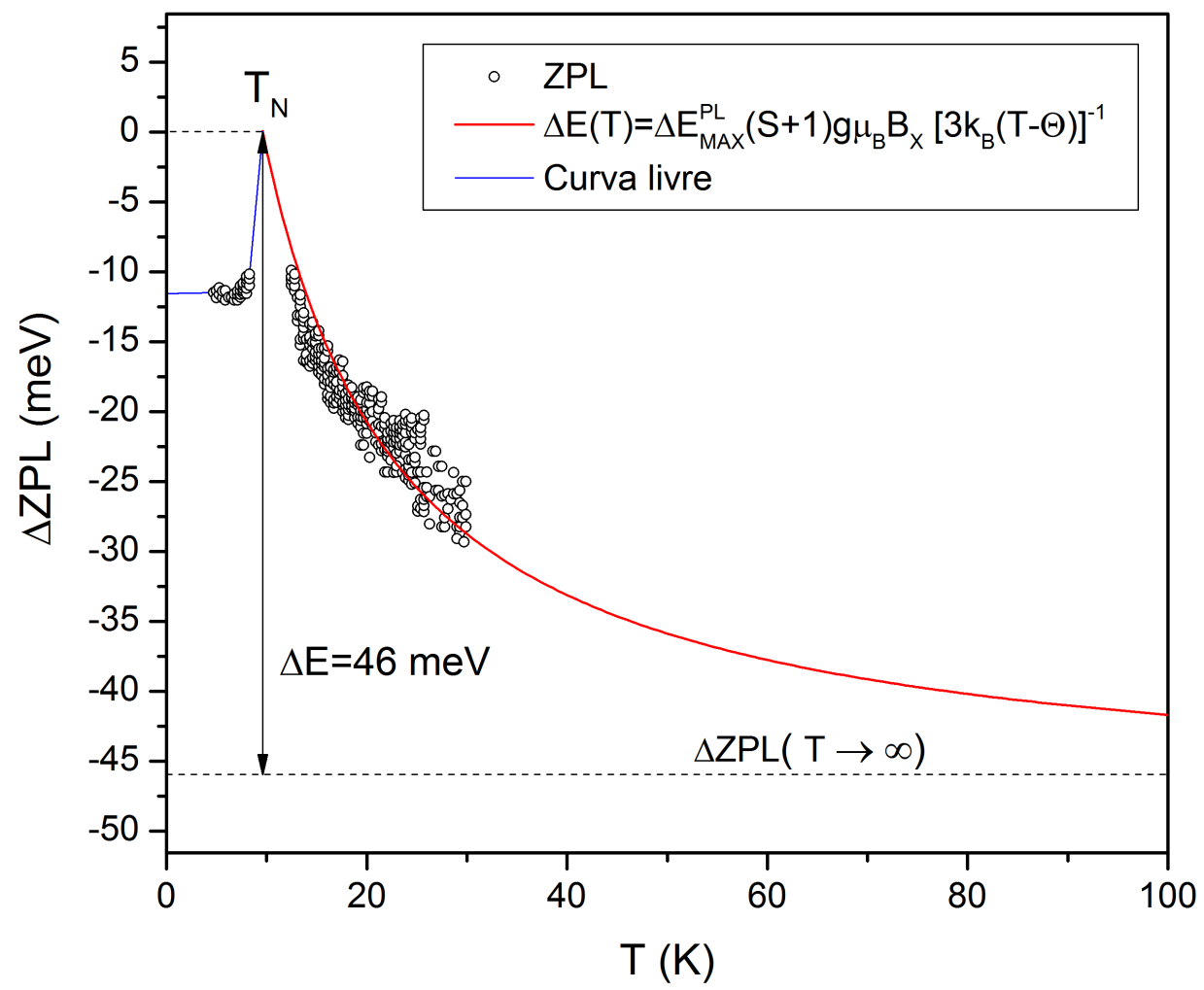

Figura 4.48: Através do ajuste da Lei de Curie-Weiss no deslocamento da ZPL em $T>T_{N}$, a energia de ligação do polaron foi estimada como $E_{p}=46 \mathrm{meV}$.

Através do deslocamento da ZPL em função da temperatura, foi obtida uma energia de ligação equivalente àquela obtida na equação (4.221) através do valor experimental do campo efetivo do elétron fotoexcitado. Esse resultado consolida a interpretação das 
observações experimentais da fotoluminescência do EuTe nas proximidades do gap óptico baseada na formação de polarons magnéticos.

\subsubsection{A natureza do estado $X$}

Através dos resultados das medidas da PL em função do campo e da temperatura, foi possível concluir que a presença do elétron fotoexcitado num estado relaxado $X$ induz a formação de um polaron magnético, cujos parâmetros foram derivados e confirmados pela medida em função da temperatura. Entretanto todos esses resultados foram obtidos sem a necessidade de saber exatamente qual é o estado eletrônico $X$ que o elétron fotoexcitado ocupa após a relaxação. Nesta seção, procuramos determinar qual é a natureza desse estado.

Uma possível ideia para a origem da luminescência $\mathrm{MX}_{0}$ é que essa possa ser associada a um estado $6 s$, entretanto a transição de dipolo elétrico entre um estado eletrônico s e um estado $f$ é proibida, de forma que o estado $X$ não pode ser um estado $s$ puro. Um estado híbrido, $s d$, por exemplo, poderia ser o estado $X$, o qual teria uma probabilidade baixa na absorção, justificando porque nenhuma absorção é observada na energia da $\mathrm{MX}_{0}$. A probabilidade de emissão entretanto, seria maior, uma vez que o estado $X$ é um estado de mínima energia, porém o tempo de vida da PL $\mathrm{MX}_{0}$ de 1 ns [91], indica que essa transição não deve ser associada a uma transição parcialmente proibida, que teria associada a si um tempo característico muito mais longo, de forma que a hipótese de que a banda $\mathrm{MX}_{0}$ advenha de um estado intrínseco composto por orbital $s$ deve ser deixada de lado.

Uma outra possibilidade é que a diferença entre a energia do gap de absorção e das linhas de emissão, esteja associada à formação de um éxciton, que reduziria a energia do elétron fotoexcitado por uma quantidade equivalente à sua energia de ligação. Para estimar essa energia de ligação podemos seguir a abordagem do modelo de Bohr utilizada na referência [94], que resulta em uma energia de ligação do éxciton no estado fundamental dada por

$$
E=-\frac{\mu}{m_{e}} \frac{1}{\varepsilon^{2}} R_{y}
$$


onde $\mu=\frac{m_{e}^{*}+m_{h}^{*}}{m_{e}^{*} m_{h}^{*}}$ é a massa reduzida do sistema elétron buraco, $\varepsilon \simeq 6$ é a constante dielétrica do EuTe (obtida na referência [19]) e $R_{y}=-13.6 \mathrm{eV}$ é a constante de Rydberg. Utilizando as massas efetivas $m_{e}^{*}=0.37 m_{e}$ dada na referência [36] e $m_{h}^{*} \rightarrow \infty$ obtemos uma energia de ligação $E \simeq 140 \mathrm{meV}$, que é muito pequena comparada ao desvio de Stokes de $\sim 400 \mathrm{meV}$. Ademais o espectro de absorção do EuTe não apresenta nenhuma evidência de formações excitônicas, portanto a hipótese de que o estado $X$ seja associado a um par elétron-buraco pode ser descartada.

A possibilidade que nos pareceu mais plausível, surgiu da análise da figura 4.49, que mostra a intensidade da luminescência em função da potência de excitação. Para baixa potência de excitação, apenas a luminescência $\mathrm{MX}_{0}$ é observada, e sua intensidade cresce sublinearmente com a potência de excitação. Por outro lado, quando a PL é dominada por $\mathrm{MX}_{1}$ e $\mathrm{MX}_{2}$, a intensidade cresce linearmente com a potência de excitação. Isso é sugestivo de que a partir de certa potência de excitação, os estados luminescentes que geram a banda $M \mathrm{X}_{0}$ tenham suas populações saturadas, de forma a partir dessa potência de excitação, o aumento do número de fótons incididos causa um aumento nas intensidades de $\mathrm{MX}_{1}$ e $\mathrm{MX}_{2}$ apenas. Ajustando uma reta à curva da intensidade da PL em função da potência de excitação na região de altas potências, podemos decompor a intensidade total da PL em duas curvas, uma para $\mathrm{MX}_{0}$ e outra para $\mathrm{MX}_{1}+\mathrm{MX}_{2}$, assumindo que a intensidade de $M X_{1}$ e $M X_{2}$ cresça linearmente com a potência de excitação desde baixas até altas potências. O resultado dessa decomposição são as linhas tracejadas na figura 4.49. Observamos que a intensidade de $\mathrm{MX}_{0}$ é caracterizada por uma saturação acima de uma potência de excitação da ordem de $0.5 \mathrm{~W} / \mathrm{cm}^{2}$. Esse comportamento é característico de estado eletrônicos luminescentes associados a defeitos: a intensidade limite ocorre quando todos os defeitos já estão populados com elétrons fotoexcitados. Esta hipótese é apoiada pela não-observação de qualquer absorção óptica na energia do fóton de luminescência. De fato a densidade de estados associada aos defeitos pode estar abaixo do limiar detectável na absorção, porém esses estados canalizam com muita eficiência a recombinação responsável pela fotoluminescência, justamente por serem estados de mínima energia. 


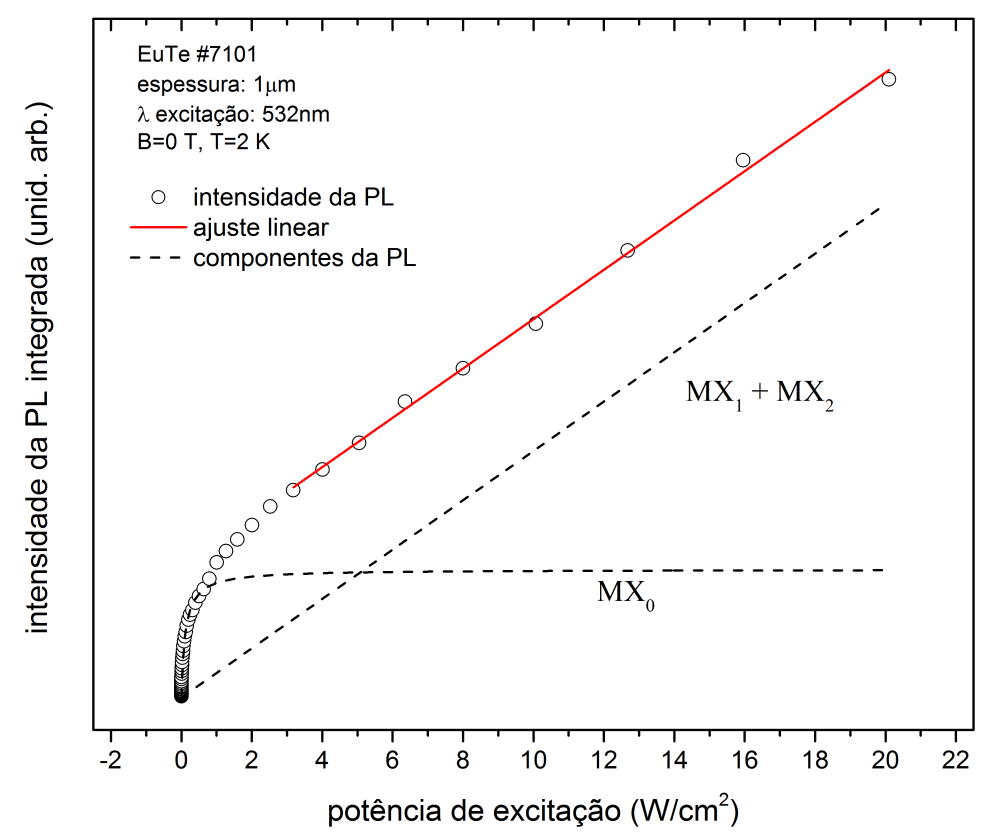

Figura 4.49: Intensidade da fotoluminescência em função da potência de excitação.

Ainda no intuito de entender qual a origem do estado $X$, foi realizado um estudo acerca da intensidade da fotoluminescência em função da temperatura. Na figura 4.50 observamos as curvas de PL geradas com potência de excitação de $24 \mathrm{~mW} / \mathrm{cm}^{2}$ para algumas temperaturas diferentes. A potência de excitação utilizada é suficiente para que a linha $\mathrm{MX}_{1}$ seja excitada, o que fica claro nas curva com $\mathrm{T}$ a partir de $13 \mathrm{~K}$. Foi feito um ajuste das curvas da $\mathrm{PL}$, somando-se a banda $\mathrm{MX}_{0}$, modelada por uma distribuição de gaussianas igualmente espaçadas e de intensidades (4.200) e largura 15 meV, com a banda $\mathrm{MX}_{1}$, modelada por uma gaussiana de largura $8 \mathrm{meV}$, de onde foi possível obter com precisão as intensidades de $\mathrm{MX}_{0}$ e $\mathrm{MX}_{1}$ isoladamente. Essas intensidades estão apresentadas na figura 4.51 a), onde percebemos que ambas comportam-se de maneira semelhante, evidenciando uma diminuição com o aumento da temperatura. Entretanto se olharmos para as intensidades relativas, definidas como

$$
I_{0}^{R}=\frac{I\left(M X_{0}\right)}{I\left(M X_{0}\right)+I\left(M X_{1}\right)} \quad \text { e } \quad I_{1}^{R}=\frac{I\left(M X_{1}\right)}{I\left(M X_{0}\right)+I\left(M X_{1}\right)}
$$

e apresentadas na figura $4.51 \mathrm{~b}$ ), podemos notar que $I_{0}^{R}$ diminui com o aumento da temperatura, ao passo que $I_{1}^{R}$ aumenta com o aumento da temperatura. Isso pode ser 


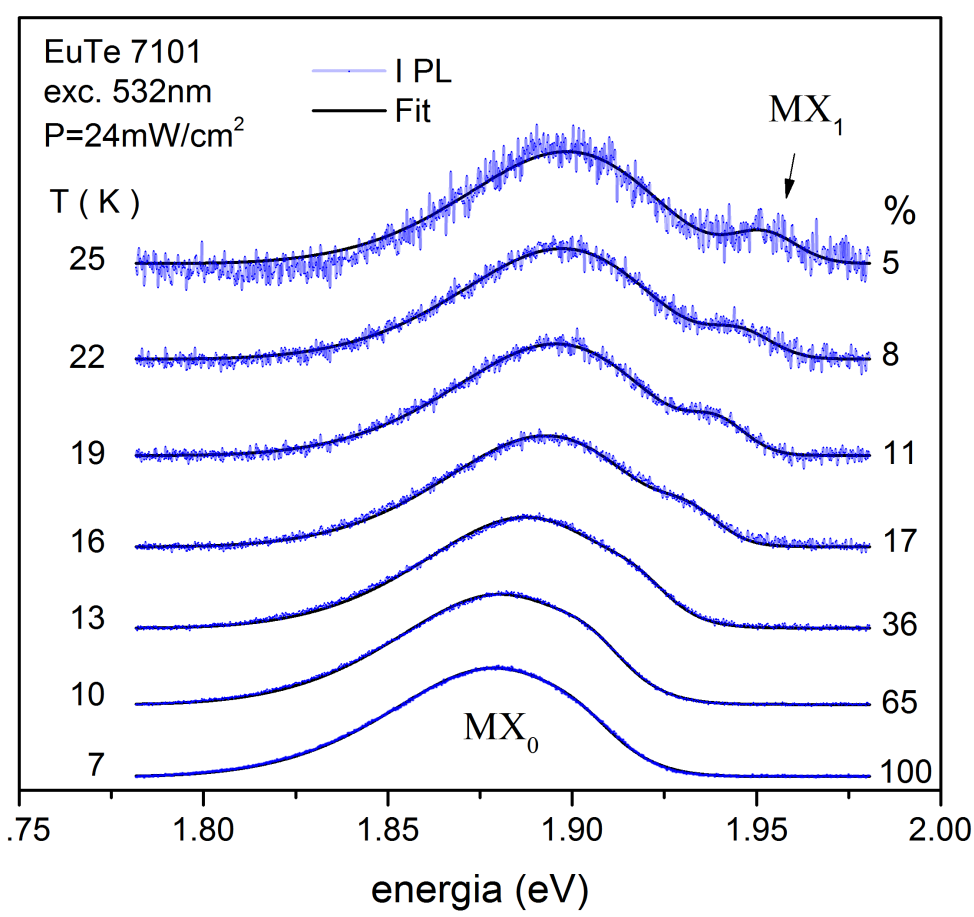

Figura 4.50: Espectros de PL em função da temperatura.

compreendido pelo fato de que os estados associados à $\mathrm{PL} \mathrm{MX}$ encontram-se saturados para a potência de excitação utilizada, portanto a introdução de energia térmica no sistema não pode favorecer o aumento da população dos estados associados à banda $\mathrm{MX}_{0}$, ao contrário do que ocorre com a linha $\mathrm{MX}_{1}$.

Para buscar informações adicionais acerca da natureza desse estado, foram realizadas medidas da fotoluminescência de excitação (PLE), em função do campo e da temperatura. Os espectros de PLE consistem em obter o espectro de PL com diferentes comprimentos de onda de excitação. A integral da curva de PL em cada comprimento de onda de excitação fornece um ponto na curva de PLE, de forma que é possível verificar em quais energias de excitação a PL é mais ou menos eficiente. Evidentemente abaixo do gap de absorção a intensidade da PLE deve ser nula e acima do gap o espectro depende do coeficiente de absorção e do caminho que o elétron fotoexcitado percorre antes de recombinar com o buraco emitindo um fóton. Esses espectros foram obtidos para amostras de diferentes espessuras e com controle de polarização da luz de excitação e estão apresentados na figura 4.52. O primeiro fato intrigante é que os 


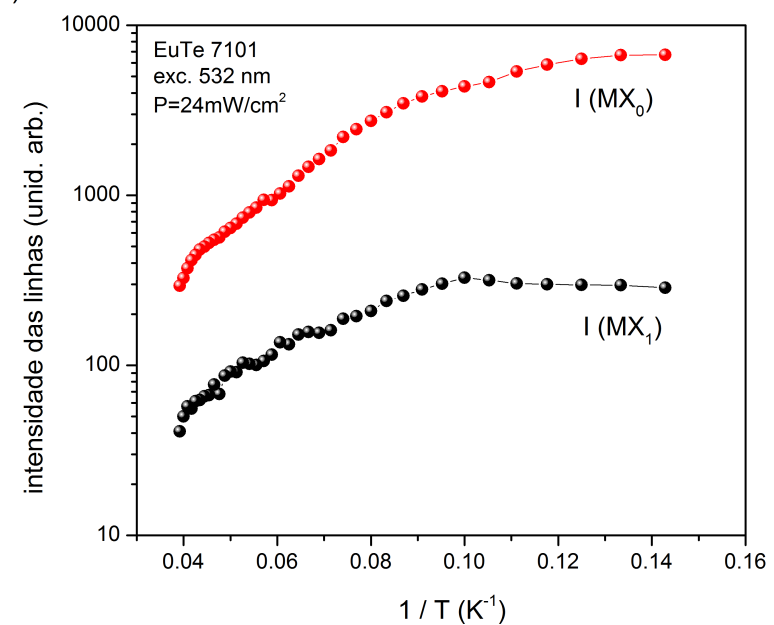

b)

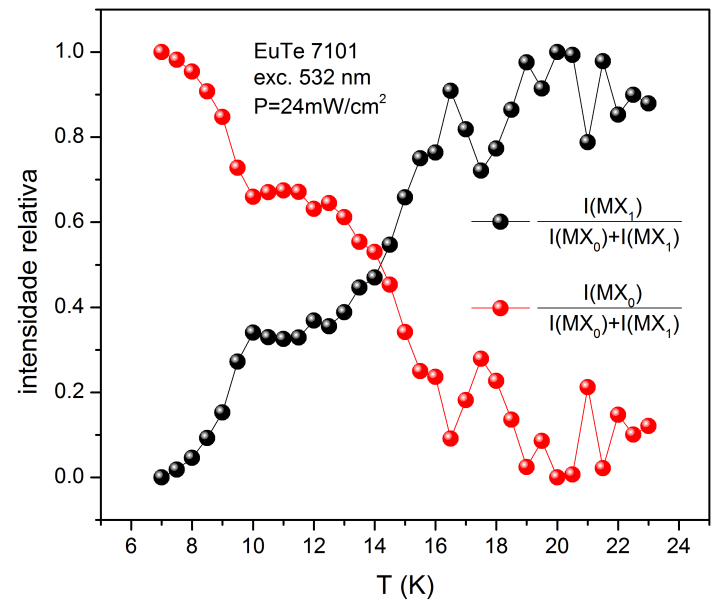

Figura 4.51: Intensidade das linhas de PL, a) em escala logarítmica b) intensidades relativas.
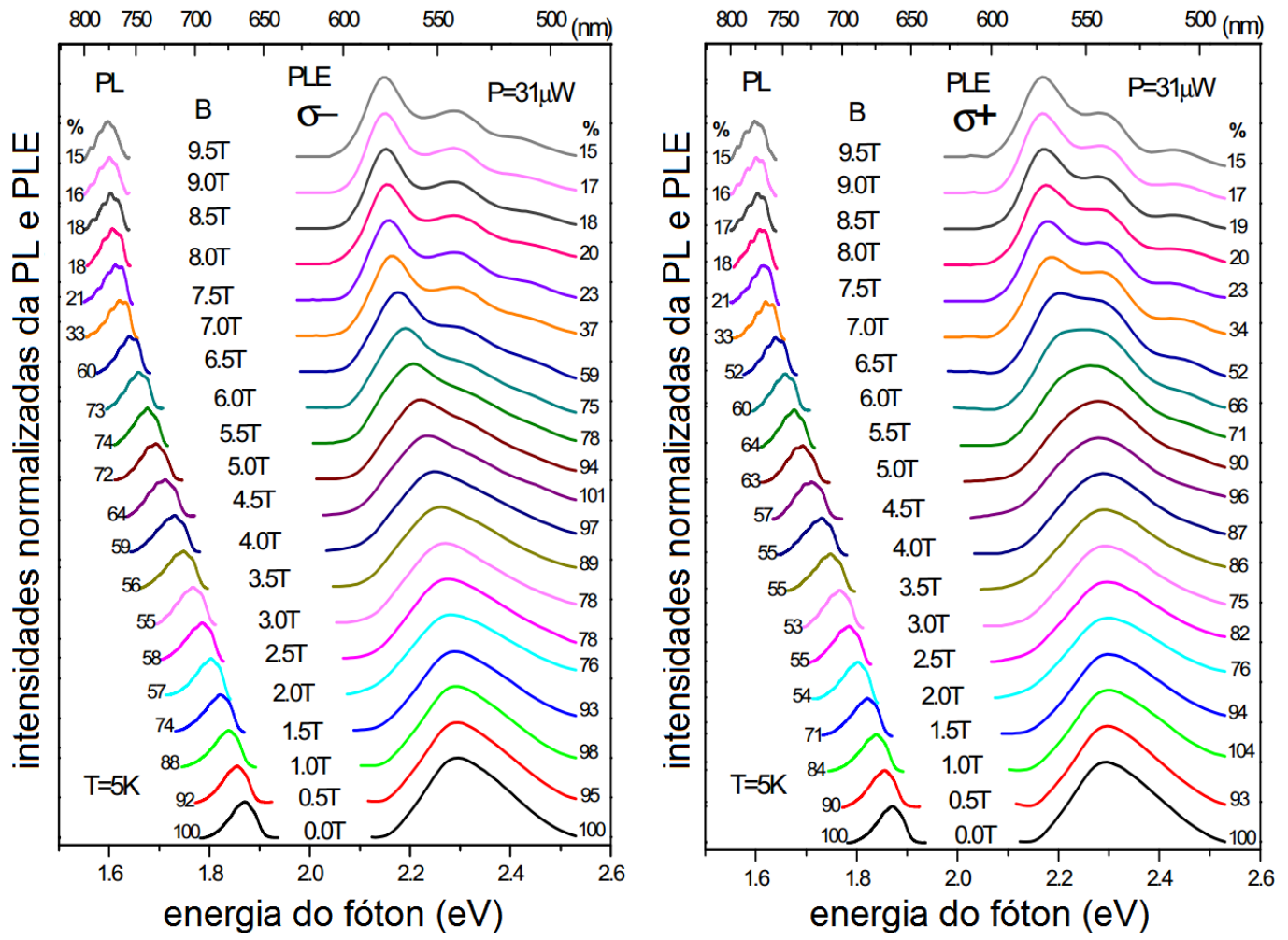

Figura 4.52: Espectros de PLE em função do campo magnético para as duas polarizações circulares da luz de excitação. 
espectros de PLE apresentam um máximo de intensidade, o que indica que a partir de certa energia, determinada por esse máximo, a relaxação do elétron, desde o estado $5 d\left(t_{2 g}\right)$ até o estado $X$, torna-se menos provável, sugerindo que este elétron encontre outros caminhos para retornar ao estado fundamental, que não a emissão de um fóton. Esse máximo desloca-se para o vermelho com o aumento do campo magnético, o que implica que a excitação ressonante é deslocada para energias de excitação menores e não que a transição torna-se proibida em campo alto como sugerido na referência [85], onde foi utilizada uma energia de excitação fixa em $532 \mathrm{~nm}(\sim 2.33 \mathrm{eV})$, para a qual a luminescência torna-se pouco eficiente. Combinado com o fato de que o sinal cai para cerca de $15 \%$ da intensidade em campo zero, é possível compreender porque foi suposto que a PL teria sido extinguida.

Também podemos observar na figura 4.52 vemos que os espectros de PLE para as polarizações LHCP e RHCP da luz incidente são muito parecidos, apresentando um dicroísmo muito pequeno em comparação ao enorme dicroísmo observado na absorção. Isso revela que o elétron no estado $X$ é fotogerado com a mesma eficiência para excitações produzidas com fótons $\sigma^{+}$ou $\sigma^{-}$. De fato, apesar dos coeficientes de absorção $\alpha^{+}$e $\alpha^{-}$serem muito diferentes, a densidade óptica $\alpha^{ \pm} d$, para as amostras de espessura $d \gtrsim 0.3 \mu \mathrm{m}$ é por demais alta na região $h v>2.3 \mathrm{eV}$, e toda a luz incidente é absorvida pela amostra, seja ela $\sigma^{+}$ou $\sigma^{-}$. Uma vez absorvida, é de se esperar uma eficiência igual na luminescência $\mathrm{MX}_{0}$ para os dois tipos de luz circularmente polarizada. O pequeno dicroísmo observado nos espectros da figura 4.52 pode ser associado à ocorrência da absorção em diferentes profundidades do material para $\sigma^{+} \mathrm{e}$ $\sigma^{-}$, dada a diferença entre $\alpha^{+}$e $\alpha^{-}$. Logo o elétron será fotogerado em regiões onde a densidade de defeitos é diferente: a densidade de defeitos geralmente é diferente em diferentes profundidades da amostra, pois na região próxima ao substrato, a diferença entre os parâmetros de rede do EuTe e do $\mathrm{BaF}_{2}$ causa um desencontro entre as estruturas cúbicas dos dois materiais, que acomodam-se em conformações por assim chamadas defeituosas. Em regiões afastadas do substrato, a influência do desencaixe é menor, de forma que a densidade de defeitos é menor nessas regiões, o que é representado na 
figura 4.53.

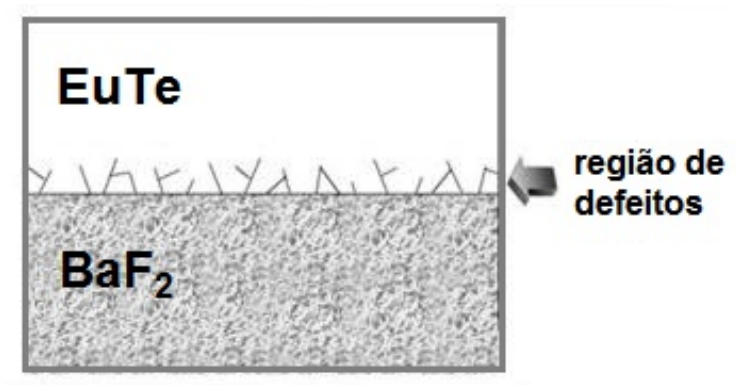

Figura 4.53: No crescimento epitaxial de EuTe em substratos de $\mathrm{BaF}_{2}$, a diferença nos parâmetros de rede, de cerca de $6 \%$, faz com que nas regiões próximas à interface ocorra densidade de defeitos maior do que nas regiões mais afastadas do substrato.

A partir da potência de saturação obtida pelo ajuste da figura 4.49, é possível obter uma estimativa da densidade de defeitos, como se segue. A densidade de centros emissores, $n$, é determinada pela equação

$$
\frac{d n}{d t}=-\frac{n}{\tau}+G
$$

onde $\tau$ é o tempo de vida do estado $X$ e $G$ é o número de centros emissores gerados por unidade de tempo e de volume. No regime estacionário, temos

$$
\frac{d n}{d t}=0 \rightarrow n=G \tau
$$

Quando ilumina-se o material com a potência de saturação, todos os defeitos estão populados. Considerando-se que nenhum outro estado é populado, a densidade de defeitos, $n_{D}$, corresponde à densidade de centros emissores excitados $n$, de forma que

$$
n_{D}=G_{S A T} \tau
$$

onde $G_{S A T}$ é a taxa de geração de saturação.

Para calcularmos $G_{S A T}$, consideramos que em um intervalo de tempo de $1 \mathrm{~s}$, a energia de um feixe de luz de potência $P$ que incide em uma área $A$ da amostra é dada por

$$
\frac{P \times 1 \mathrm{~s}}{A}=n_{\phi} \times A \times c \times h v
$$


onde $n_{\phi}$ é a densidade de fótons no feixe, $c=3 \times 10^{8} \mathrm{~m}$ é o espaço percorrido pela luz em $1 \mathrm{~s}$ e $h v$ é a energia de cada fóton. Em termos da intensidade $I$ do feixe, podemos exprimir (4.245) como

$$
n_{\phi} \times A \times c \times h v=I A
$$

de forma que

$$
n_{\phi}=\frac{I}{c h v}
$$

A taxa de geração é dada pela densidade de fótons que penetram um volume $V$ da amostra, multiplicado pela eficiência quântica, definida como

$$
\chi=\frac{N_{G}}{N_{I}}
$$

onde $N_{G}$ é o número de fótons que geram um centro emissor e $N_{I}$ é o número de fótons incidentes. Evidentemente $\chi$ deve ser um número menor ou igual à unidade.

A energia incidente é totalmente absorvida pelo material em uma profundidade típica da ordem de $1 / \alpha$, onde $\alpha$ é o coeficiente de absorção do material. Assim, a densidade de fótons absorvidos pelo material é dada pelo número de fótons incidentes em um segundo, dado por $n_{\phi} \times A \times c$, dividido pelo volume onde esses fótons são absorvidos, dado por $A \times 1 / \alpha$, de forma que a taxa de geração é dada por

$$
G=\chi \frac{n_{\phi} \times A \times c}{A \times \frac{1}{\alpha}} \underbrace{=}_{(4.247)} \chi \frac{I \alpha}{h v}
$$

de forma que $G_{S A T}=\chi \frac{I_{S A T \alpha}}{h v}$. Substituindo este resultado na equação (4.244), obtemos a densidade de defeitos

$$
n_{D}=G_{S A T} \tau=\chi \frac{I_{S A T} \alpha}{h v} \tau
$$

a qual com os valores $I_{S A T}=0.5 \mathrm{~W} / \mathrm{cm}^{2}$ obtido através do ajuste da figura $4.49, \alpha=15 \mu \mathrm{m}^{-1}$ obtido dos espectros de absorção para $\lambda=532 \mathrm{~nm}, \tau \sim 5 \mathrm{~ns}$ determinado a partir de mediadas da PL resolvida no tempo [91] e $h v=3.7 \times 10^{-19} \mathrm{~J}$ obtemos

$$
n_{D}=\chi 3 \times 10^{15} \mathrm{~cm}^{-3} \rightarrow n_{D} \leq 3 \times 10^{15} \mathrm{~cm}^{-3}
$$


o que corresponde a uma concentração relativa máxima de um defeito para cada $10^{7}$ átomos do material, ou 0.1 ppm.

Com a soma de todos os fatores apresentados nesta seção, ou seja, a saturação da PL $\mathrm{MX}_{0}$ com o aumento da potência de excitação, a presença de um dicroísmo circular magnético e a eliminação da possibilidade de estados intrínsecos como uma banda de baixa densidade de estados ou um éxciton magnético, podemos concluir que as linhas $\mathrm{MX}_{0}$ são oriundas de estados associados a defeitos. 


\section{Capítulo 5}

\section{Conclusões}

A partir do modelo da transição óptica $4 f \rightarrow 5 d\left(t_{2 g}\right)$ foi possível explicar totalmente o espectro de absorção dos EuX em função do campo magnético; em campo alto, a presença de linhas estreitas de absorção é bem descrita pelo modelo e em campo nulo, a formação de domínios magnéticos explica a não-observação de linhas estreitas nos espectros de absorção.

A partir dos espectros de absorção foi possível detectar as fases magnéticas do EuSe, evidenciando como é possível utilizar uma técnica óptica para a determinação do estado magnético de um material, o que eventualmente pode ser aproveitado em aplicações tecnológicas.

Nos espectros de fotoluminescência do EuTe, foram detectados estados eletrônicos não contemplados pelo modelo $4 f \rightarrow 5 d\left(t_{2 g}\right)$. Foi investigada em especial a banda de PL $\mathrm{MX}_{0}$, observada em baixas potências de excitação. Foi observado que essa banda surge de um acoplamento da transição óptica com modos vibracionais da rede. Foram determinadas a força desse acoplamento, a largura das linhas individuais e o espaçamento entre as linhas, que corresponde à energia do fônon-óptico longitudinal no EuTe.

As linhas muito estreitas $(\delta \sim 8 \mathrm{~nm})$ e oriundas de estados eletrônicos puros, permitiram uma caracterização precisa da PL do EuTe. Através do deslocamento da ZPL em função do campo magnético foi possível detectar a formação de polarons magnéticos, 
evidenciada pela redução da magnitude do campo magnético que deve ser aplicada para alinhar os spins da rede. Essa diferença foi associada a um campo efetivo criado pelo elétron fotoexcitado, que alinha os spins da rede, criando uma região de spins polarizados, ou um polaron magnético. A partir do deslocamento da ZPL em função do campo foi possível estimar o raio desse polaron $\operatorname{como} R=3.6 a$, onde $a$ é o parâmetro de rede, e a energia de ligação como $E_{p}=45 \mathrm{meV}$, o que foi confirmado por medidas do deslocamento da ZPL em função da temperatura.

O estado eletrônico que dá origem à formação do polaron magnético foi investigado. A partir do comportamento da intensidade da luminescência em função da potência de excitação, deduzimos que o estado eletrônico está associado à presença de defeitos de concentração muito baixa, da ordem de $\sim 10^{15} \mathrm{~cm}^{-3}$, ou $\sim 0.1 \mathrm{ppm}$.

A formação de polarons magnéticos em decorrência da excitação óptica no EuTe, demonstra a possibilidade de gerar uma magnetização num composto AFM através da incidência de radiação, um fenômeno que pode ser aprofundado e estudado, no âmbito do crescente interesse em controlar o magnetismo nos materiais através da luz, seja para a aplicação em dispositivos tecnológicos, seja para o desenvolvimento da nova área da física conhecida como opto-magnetismo. 


\section{Bibliografia}

[1] T. Jenkins, A Brief History of ...Semiconductors, Physics Education 40, 430 (2005). $(\leftarrow$ pg. 17).

[2] S. A. Wolf et al., Spintronics: A Spin-Based Electronics Vision for the Future, Science 294, 1488 (2001). ( $\leftarrow$ pg. 17).

[3] I. Žutić, J. Fabian, and S. Das Sarma, Spintronics: Fundamentals and applications, Rev. Mod. Phys. 76, 323 (2004). ( $\leftarrow$ pg. 17).

[4] A. H. MacDonald, N. Samarth, and P. Schiffer, Ferromagnetic semiconductors: moving beyond (Ga,Mn)As, Nature Materials 4, 195 (2005). ( $\leftarrow$ pg. 18).

[5] W. Klemm and H. Senff, Messungen an zwei- und vierwertigen Verbindungen der seltenen Erden. VIII. Chalkogenide des zweiwertigen Europiums, Zeitschrift für anorganische und allgemeine Chemie 241, 259 (1939). ( $\leftarrow$ pg. 18).

[6] T. R. McGuire, B. E. Argyle, M. W. Shafer, and J. S. Smart, Ferromagnetism in divalent europium salts, Applied Physics Letters 1, 17 (1962). ( $\leftarrow$ pgs. 18, 63).

[7] U. Enz, J. Fast, S. v. Houten, and J. Smit, Phillips Res. Repts. 17, 451 (1962). ( $\leftarrow$ pgs. $18,54)$.

[8] S. Methfessel, Potential applications of magnetic rare earth compunds, Magnetics, IEEE Transactions on 1, 144 (1965). ( $\leftarrow$ pg. 18).

[9] G. Güntherodt, P. Wachter, and D. Imboden, Energy level scheme and the effect of magnetic order on the optical transitions in europium chalcogenides, Zeitschrift für 
Physik B Condensed Matter 12, 292 (1971), 10.1007/BF02422557. ( $\leftarrow$ pgs. 18, 19, 54).

[10] J. O. Dimmock, Optical Properties of Europium Chalcogenides, IBM J. Res. Dev. 14, 301 (1970). (Łpg. 18).

[11] T. S. Santos et al., Determining Exchange Splitting in a Magnetic Semiconductor by Spin-Filter Tunneling, Phys. Rev. Lett. 101, 147201 (2008). ( $\leftarrow$ pg. 18).

[12] J. F. Gregg, Spintronics: A growing science, Nat. Mater. 6, 798 (2007). ( $\leftarrow$ pg. 18).

[13] M. D. Regulacio, K. Bussmann, B. Lewis, and S. L. Stoll, Magnetic properties of lanthanide chalcogenide semiconducting nanoparticles, Journal of the American Chemical Society 128, 11173 (2006). ( $\leftarrow$ pg. 18).

[14] M. L. Redígolo, D. S. Koktysh, K. van Benthem, S. J. Rosenthal, and J. H. Dickerson, Europium sulfide nanoparticles in the sub-2 $\mathrm{nm}$ size regime, Materials Chemistry and Physics 115, 526 (2009). ( $\leftarrow$ pg. 18).

[15] D. S. Koktysh et al., EuS nanocrystals: a novel synthesis for the generation of monodisperse nanocrystals with size-dependent optical properties, Nanotechnology 21, 415601 (2010). (Łpg. 18).

[16] W. He, S. Somarajan, D. S. Koktysh, and J. H. Dickerson, Superantiferromagnetic EuTe nanoparticles: room temperature colloidal synthesis, structural characterization, and magnetic properties, Nanoscale 3, 186 (2011). ( $\leftarrow$ pg. 18).

[17] T. U. Schulli et al., Soft x-ray magnetic scattering from ordered EuSe nanoislands, Applied Physics Letters 84, 2661 (2004). ( $\leftarrow$ pg. 18).

[18] B. Díaz et al., Growth of EuTe islands on SnTe by molecular beam epitaxy, Journal of Crystal Growth 312, 2828 (2010). ( $\leftarrow$ pg. 18).

[19] P. Wachter, Handbook on the Physics and Chemistry of Rare Earths vol. 1, NorthHolland, 1979. ( $\leftarrow$ pgs. 18, 72, 84, 104, 139, 141, 171, 173). 
[20] T. Kasuya, Energy Spectra of Magnetic Semiconductors: Eu Chalcogenides and NiO, Journal of Applied Physics 41, 1090 (1970). $(\leftarrow$ pg. 18).

[21] G. Güntherodt, Optical properties and electronic structure of europium chalcogenides, Zeitschrift für Physik B Condensed Matter 18, 37 (1974), 10.1007/BF01950500. $(\leftarrow$ pgs. 19, 54).

[22] A. B. Henriques et al., Tuning of The Magnetic Field Induced Sharp Band-Edge Optical Absorption in Europium Chalcogenides, International J. of Mod. Phys. B 18, 3813 (2004). (Łpg. 19).

[23] L. K. Hanamoto et al., Sharp lines in the absorption edge of EuTe and Pb 0.1 Eu 0.9 Te in high magnetic fields, Journal of Physics: Condensed Matter 16, 5597 (2004). ( $\leftarrow$ pg. 19).

[24] A. B. Henriques et al., Band-edge polarized optical absorption in europium chalcogenides, Phys. Rev. B 72, 155337 (2005). ( $\leftarrow$ pgs. 19, 72, 135).

[25] L. K. Hanamoto et al., Magnetic field induced absorption in $\mathrm{Pb}_{x} E u_{1-x}$ Te magnetic semiconductors, Brazilian Journal of Physics 34, 687 (2004). ( $\leftarrow$ pg. 19).

[26] A. B. Henriques, G. D. Galgano, B. Díaz, P. Rappl, and E. Abramof, Magnetic field dependence of the circular dichroism in EuTe, Journal of Physics: Condensed Matter

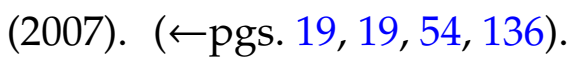

[27] A. B. Henriques, M. A. Manfrini, P. H. O. Rappl, and E. Abramof, Modeling the dichroic absorption band edge and light-induced magnetism in EuTe, Phys. Rev. B 77, 035204 (2008). (Łpg. 19).

[28] A. Henriques, G. D. Galgano, and A. E., Magnetic linear dichroism in absorption spectroscopy of EuTe, Journal of Physics: Condensed Matter (2008). ( $\leftarrow$ pgs. 19, 127, 133). 
[29] A. B. Henriques, E. Abramof, and P. H. O. Rappl, Theory of near-gap second harmonic generation in centrosymmetric magnetic semiconductors: Europium chalcogenides, Phys. Rev. B 80, 245206 (2009). ( $\leftarrow$ pg. 19).

[30] B. Kaminski et al., Spin-Induced Optical Second Harmonic Generation in the Centrosymmetric Magnetic Semiconductors EuTe and EuSe, Phys. Rev. Lett. 103, 057203 (2009). (Łpg. 20).

[31] B. Kaminski et al., Optical second harmonic generation in the centrosymmetric magnetic semiconductors EuTe and EuSe, Phys. Rev. B 81, 155201 (2010). ( $\leftarrow$ pg. 20).

[32] M. Lafrentz et al., Optical third-harmonic spectroscopy of the magnetic semiconductor EuTe, Phys. Rev. B 82, 235206 (2010). ( $\leftarrow$ pg. 20).

[33] M. Lafrentz et al., Optical third harmonic generation in the magnetic semiconductor EuSe, Phys. Rev. B 85, 035206 (2012). ( $\leftarrow$ pg. 20).

[34] W. Nolting, Modified sf model for mixed-valence Eu chalcogenides, J. Phys. C: Solid State Phys. 15, 773 (1982). ( $\leftarrow$ pg. 20).

[35] A. Mauger and C. Godart, The magnetic, optical, and transport properties of representatives of a class of magnetic semiconductors: The europium chalcogenides, Physics Reports 141, 51 (1986). ( $\leftarrow$ pg. 20).

[36] M. Umehara, Photoinduced localized magnetic polaron and luminescence in Eu chalcogenides, especially in EuTe, Phys. Rev. B 52, 8140 (1995). ( $\leftarrow$ pgs. 20, 162, 173).

[37] A. S. Davydov, Quantum Mechanics, Pergamon, 1965. ( $\leftarrow$ pgs. 24, 35, 115).

[38] C. J. Foot, Atomic Physics, Oxford, 2005. ( $\leftarrow$ pg. 26).

[39] N. W. Ashcroft and D. N. Mermin, Solid State Physics, Harcourt, 1976. ( $\leftarrow$ pgs. 27, 38).

[40] J. Stöhr and H. C. Siegmann, Magnetism From Fundamentals to Nanoscale Dynamics, Springer, 2006. ( $\leftarrow$ pgs. 27, 29). 
[41] C. Kittel, Quantum Theory of Solids, John Wiley \& Sons, 1963. ( $\leftarrow$ pg. 27).

[42] P. Weiss, L'hypothèse du champ moléculaire et la propriété ferromagnétique, Journal de Physique Théorique et Appliquée (1907). ( $\leftarrow$ pg. 30).

[43] W. Heisenberg, Mehrkörperproblem und Resonanz in der Quantenmechanik, Zeitschrift für Physik A Hadrons and Nuclei 38, 411 (1926), 10.1007/BF01397160. ( $\leftarrow$ pg. 30).

[44] W. Heisenberg, Zur Theorie des Ferromagnetismus, Zeitschrift für Physik A Hadrons and Nuclei 49, 619 (1928). ( $\leftarrow$ pg. 30).

[45] S. Blundell, Magnetism in Condensed Matter, Oxford, 2001. ( $\leftarrow$ pgs. 31, 59).

[46] H. Goldstein, C. Poole, and J. Safko, Classical Mechanics, Addison Wesley, 2000. $(\leftarrow$ pg. 32).

[47] W. Kaplan, Advanced Calculus, Addison Wesley, fourth edition, 1991. ( $\leftarrow$ pg. 33).

[48] M. Getzlaff, Fundamentals of Magnetism, Springer, 2008. ( $\leftarrow$ pg. 42).

[49] L. Néel, Influence des fluctuations du champ moléculaire sur les propriétés magnétiques des corps, Ann. de Phys. 17, 5 (1932). ( $\leftarrow$ pg. 48).

[50] N. Kurti, Selected Works of Louis Néel, Gordon and Breach Science Publishers, 1988. $(\leftarrow$ pgs. 48,51$)$.

[51] L. Néel, Propriétées magnétiques des ferrites. Férrimagnétisme et antiferromagnétisme, Ann. Phys. 3, 137 (1948). ( $\leftarrow$ pg. 51).

[52] S. Pickart and H. Alperin, Magnetic structure of EuSe, Journal of Physics and Chemistry of Solids 29, 414 (1968). ( $\leftarrow$ pg. 54).

[53] G. Busch, P. Junod, R. G. Morris, J. Muheim, and W. Stutius, Low-temperature specific heat measurements of EuSe and EuTe, Physics Letters 11,9 (1964). ( $\leftarrow$ pg. 54).

[54] G. Busch, P. Junod, and P. Wachter, Optical absorption of ferro- and antiferromagnetic europium chalcogenides, Physics Letters 12, 11 (1964). ( $\leftarrow$ pg. 54). 
[55] Busch, G., Güntherodt, G., and Wachter, P., Optical transitions and the energy level scheme of the europium chalcogenides, J. Phys. Colloques 32, C1 (1971). ( $\leftarrow$ pg. 54).

[56] J. Schoenes, Exchange and superlattice band splittings in europium chalcogenides; A magneto-optical study in high magnetic fields, Journal of Magnetism and Magnetic Materials 11, 102 (1979). ( $\leftarrow$ pg. 54).

[57] J. S. Smart, Effective Field Theories of Magnetism, London:Saunders, 1966. ( $\leftarrow$ pgs. $57,64)$.

[58] L. M. Griessen, R. and H. Ott, A new antiferromagnetic phase in EuSe below $1.8 \mathrm{~K}$, Solid State Communciations 9 (1971). ( $\leftarrow$ pgs. 63, 64, 65, 65, 66, 67, 141).

[59] B. T. Matthias, R. M. Bozorth, and J. H. Van Vleck, Ferromagnetic Interaction in EuO, Phys. Rev. Lett. 7, 160 (1961). ( $\leftarrow$ pg. 63).

[60] T. R. McGuire, B. E. Argyle, M. W. Shafer, and J. S. Smart, Magnetic Properties of Some Divalent Europium Compounds, Journal of Applied Physics 34, 1345 (1963). $(\leftarrow$ pg. 63).

[61] O. W. Dietrich, A. J. Henderson, and H. Meyer, Spin-wave analysis of specific heat and magnetization in EuO and EuS, Phys. Rev. B 12, 2844 (1975). ( $\leftarrow$ pg. 63).

[62] Callen and de Moura, Spin structures of europium selenide, Physical Review B 16 (1977). ( $\leftarrow$ pg. 64).

[63] B. Diaz et al., Magnetic ordering and transitions of EuSe studied by x-ray diffraction, Physical Review B 81 (2010). ( $\leftarrow$ pgs. 64, 65, 65).

[64] H. Fukuma, T. Suzuki, and T. Kasuya, Origin of Anomalous Phase diagram and anisotropy in EuSe, J. Mag. Magnetic Mat. 52, 7 (1985). ( $\leftarrow$ pg. 66).

[65] G. Will, S. Pickart, H. Alperein, and R. Nathans, Antiferromagnetic structure of EuTe, Journal of Physics and Chemistry of Solids 24, 1679 (1963). $(\leftarrow$ pg. 67). 
[66] J. W. Battles and G. E. Everett, Antiferromagnetic-Resonance Measurements in Europium Telluride, Phys. Rev. B 1, 3021 (1970). ( $\leftarrow$ pgs. 68, 69, 71, 72).

[67] N. F. Oliveira, S. Foner, Y. Shapira, and T. B. Reed, EuTe. I. Magnetic Behavior of Insulating and Conducting Single Crystals, Phys. Rev. B 5, 2634 (1972). ( $\leftarrow$ pgs. 68, $72,158,159)$.

[68] F. Keffer and W. O'Sullivan, Problem of Spin Arrangements in $\mathrm{MnO}$ and Similar Antiferromagnets, Phys. Rev. 108, 637 (1957). ( $\leftarrow$ pg. 69).

[69] B. Díaz et al., Magnetic resonant x-ray diffraction study of europium telluride, Physical Review B (Condensed Matter and Materials Physics) 78, 134423 (2008). ( $\leftarrow$ pg. 72).

[70] B. Díaz, P. H. O. Rappl, and E. Abramof, Molecular beam epitaxial growth of EuTe/SnTe strained superlattices, Journal of Crystal Growth 308, 218 (2007). ( $\leftarrow$ pg. 73).

[71] R. T. Lechner et al., Strain Induced Changes in the Magnetic Phase Diagram of Metamagnetic Heteroepitaxial EuSe/PbSe $e_{1-x} \mathrm{Te}_{x}$ Multilayers, Physical Review Letters 94

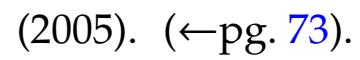

[72] P. C. Logofatu, Simple method for determining the fast axis of a wave plate, Optical Engineering 41, 3316 (2002). ( $\leftarrow$ pg. 75).

[73] G. D. Galgano and A. B. Henriques, Determining the fast axis of a wave plate, Proceedings do ENFMC (2006). ( $\leftarrow$ pg. 76).

[74] E. Hecht, Optics, Addison Wesley, 2002. ( $\leftarrow$ pg. 86).

[75] J. J. Sakurai, Modern Quantum Mechanics, Addison-Wesley, 1994. ( $\leftarrow$ pg. 92).

[76] G. B. Arfken and H. J. Weber, Mathematical Methods for Physicists, Harcourt Academic Press, 5th edition, 2001. ( $\leftarrow$ pg. 96).

[77] L. I. Schiff, Quantum Mechanics, McGraw-Hill Book Company, third edition, 1968. $(\leftarrow$ pg. 97). 
[78] L. D. Landau and E. M. Lifshitz, Quantum Mechanics: Nonrelativistic Theory, Pergamon, third edition, 1977. ( $\leftarrow$ pgs. 102, 115, 122, 124).

[79] E. U. Condon and G. H. Shortley, The Theory of Atomic Spectra, Cambridge, 1935. $(\leftarrow$ pg. 110).

[80] A. R. Edmonds, Angular Momentum in Quantum Mechanics, Princeton, 1957. ( $\leftarrow$ pg. $116,116)$.

[81] G. D. Galgano, A. B. Henriques, and E. Abramof, Magnetic Linear Dichroism Effects in EuTe, AIP Conference Proceedings 1199, 439 (2010). ( $\leftarrow$ pg. 133).

[82] W. Sollinger et al., Exchange interactions in europium monochalcogenide magnetic semiconductors and their dependence on hydrostatic strain, Physical Review B 81 (2010). (Łpgs. 139, 142).

[83] R. Kirchschlager, W. Heiss, R. T. Lechner, G. Bauer, and G. Springholz, Hysteresis loops of the energy band gap and effective g-factor up to 18000 for metamagnetic EuSe epilayers, Applied Physics Letters 85 (2004). ( $\leftarrow$ pg. 141).

[84] G. D. Galgano, A. B. Henriques, G. Bauer, and G. Springholz, Optical probing of metamagnetic phases in epitaxial EuSe, AIP Conference Proceedings 1399, 671 (2011). $(\leftarrow$ pg. 144).

[85] W. Heiss et al., Magnetic polaron induced near-band-gap luminescence in epitaxial EuTe, Phys. Rev. B 70, 035209 (2004). ( $\leftarrow$ pgs. 145, 146, 177).

[86] P. Wachter, Refractive index and dispersion of the Europium-Chalcogenides, Zeitschrift für Physik B Condensed Matter 8, 80 (1968), 10.1007/BF02422762. ( $\leftarrow$ pg. 148).

[87] P. W. Atkins, Molecular Quantum Mechanics, Oxford, third edition, 1996. $\quad(\leftarrow$ pg. 150).

[88] G. Holah, J. Webb, R. Dennis, and C. Pidgeon, Resonant Raman scattering from screened longitudinal optical phonons in EuTe, Solid State Communications 13, 209 (1973). (њpg. 150). 
[89] T. H. Keil, Shapes of Impurity Absorption Bands in Solids, Phys. Rev. 140, A601 (1965). $(\leftarrow$ pg. 151).

[90] A. Henriques and J. von der Weid, Line-shape of the vibrational bands of the CN-UV emission spectra in alkali halide-cyanide mixed crystals, Solid State Communications 56, 571 (1985). ( $\leftarrow$ pg. 151).

[91] G. D. Galgano, A. B. Henriques, B. S. L. Honda, P. C. Christianen, and A. Meaney, Time-resolved photoluminescence of EuTe in a magnetic field, a ser publicado (2012). $(\leftarrow$ pgs. 153, 172, 179).

[92] A. B. Henriques, G. D. Galgano, E. Abramof, B. Diaz, and P. H. O. Rappl, Zerophonon emission and magnetic polaron parameters in EuTe, Applied Physics Letters

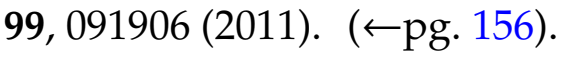

[93] A. Mauger and D. L. Mills, Role of conduction-electron - local-moment exchange in antiferromagnetic semiconductors: Ferrons and bound magnetic polarons, Phys. Rev. B 31, 8024 (1985). ( $\leftarrow$ pg. 159).

[94] M. Fox, Optical Properties of Solids, Oxford, 2001. ( $\leftarrow$ pg. 172). 


\section{Apêndice A}

\section{Lista de publicações relacionadas à tese}

- A. B. Henriques, G. D. Galgano, B. Díaz, P. H. O. Rappl e E. Abramof. Exchange interaction effects on the optical properties of $E u_{x} P b_{1-x} T e$. AIP Conference Proceedings, vol. 893, pgs. 1233-1234, 2006.

- A. B. Henriques, M. A. Manfrini, G. D. Galgano, B. L. Díaz, P. O. H. Rappl e E. Abramof. Magnetic circular dichroism in EuTe in the ferromagnetic order. International Journal of Modern Physics B, vol. 21, 8-9, pgs. 1247-1253, 2007.

- A. B. Henriques, G. D. Galgano, B. L. Díaz, P. O. H. Rappl e E. Abramof. Magnetic field dependence of the circular dichroism in EuTe. Journal of Physics: Condensed Matter, vol. 19, 406234, 2007.

- A. B. Henriques, G. D. Galgano e E. Abramof. Magnetic linear dichroism in absorption spectroscopy of EuTe. Journal of Physics: Condensed Matter, vol. 20, 255209, 2008.

- G. D. Galgano, A. B. Henriques e E. Abramof. Magnetic linear dichroism effects in EuTe. AIP Conference Proceedings, vol. 1199, 1, pgs. 439-440, 2010.

- G. D. Galgano, A. B. Henriques, G. Bauer e G. Springholz. Optical probing of metamagnetic phases in epitaxial EuSe. AIP Conference Proceedings, vol. 1399, 1, pgs. 671-672, 2011.

- A. B. Henriques, G. D. Galgano, B. L. Díaz, P. O. H. Rappl e E. Abramof. Zero-phonon emission and magnetic polaron parameters in EuTe. Applied Physics Letters, vol. 99, 9, pg. 091906, 2011. 